\title{
Wif1 Inhibits the Growth of Basal Cell Carcinoma
}

\author{
Dissertation \\ for the award of the degree \\ "Doctor rerum naturalium" \\ of the Georg-August-Universität Göttingen
}

within the doctoral program Molecular Biology of Cells

of the Georg-August University School of Science (GAUSS)

\author{
submitted by \\ Marco Becker \\ from Gelsenkirchen
}

Göttingen 2015 


\section{Thesis Committee}

\section{Prof. Dr. Heidi Hahn}

Dept. of Human Genetics; University Medical Center Göttingen

Prof. Dr. Matthias Dobbelstein

Dept. of Molecular Oncology; Georg-August-University Göttingen

Prof. Dr. Tobias Pukrop

Dept. of Hematology and Oncology; University Medical Center Regensburg

\section{Members of the Examination Board}

Referee: Prof. Dr. Heidi Hahn

Dept. of Human Genetics; University Medical Center Göttingen

Co- referee: Prof. Dr. Matthias Dobbelstein

Dept. of Molecular Oncology; Georg-August-University Göttingen

\section{Further members of the Examination Board}

\section{Prof. Dr. Holger Bastians}

Dept. of Molecular Oncology; Georg-August-University Göttingen

\section{Prof. Dr. Steven Johnsen}

Clinic for General, Visceral and Pediatric surgery; University Medical Center Göttingen

\section{Prof. Dr. Gregor Bucher}

Dept. of Developmental Biology; Georg-August-University Göttingen

\section{Prof. Dr. Sigrid Hoyer-Fender}

Dept. of Developmental Biology; Georg-August-University Göttingen 


\section{Affidavit}

\section{Affidavit}

I, Marco Becker, hereby declare that the PhD thesis entitled "Wif1 Inhibits the Growth of Basal Cell Carcinoma" has been written independently and with no other sources and aids than quoted.

Marco Becker

July, 2015

Göttingen 
"We have done it that way ever since." -absolutely everybody 


\section{Content}

\section{Content}

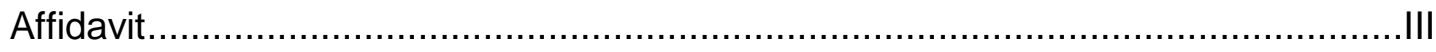

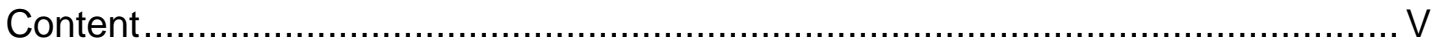

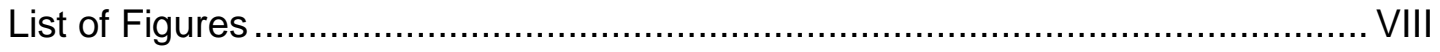

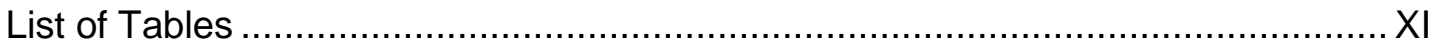

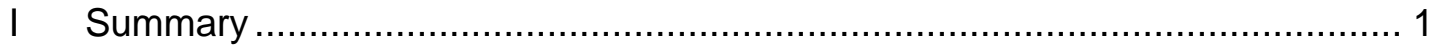

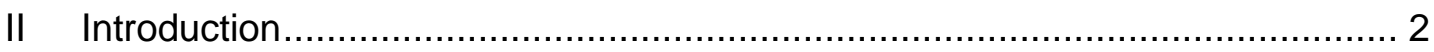

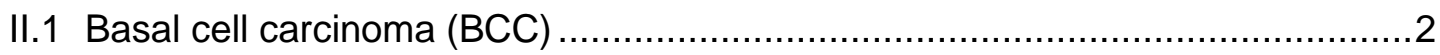

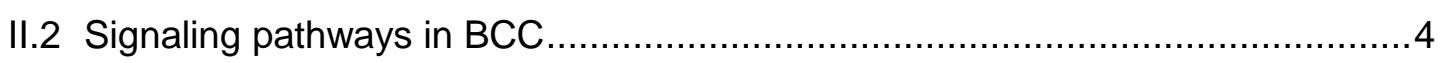

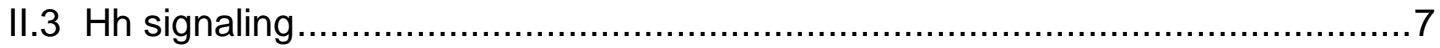

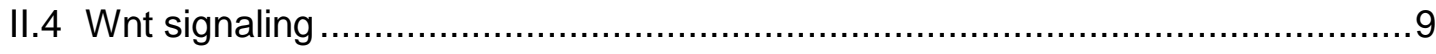

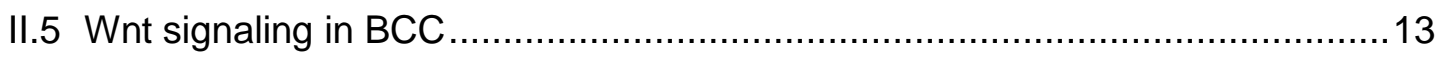

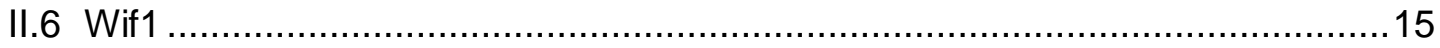

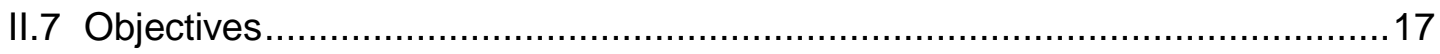

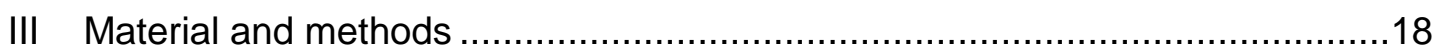

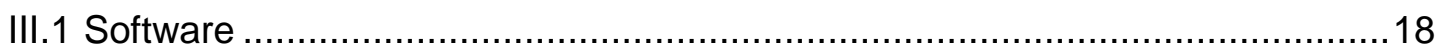

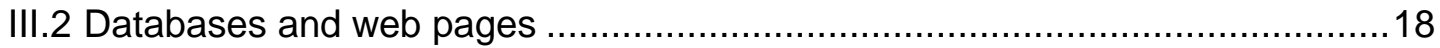

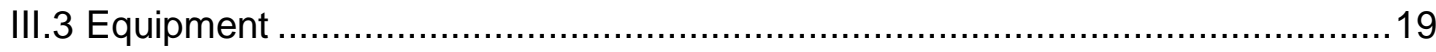

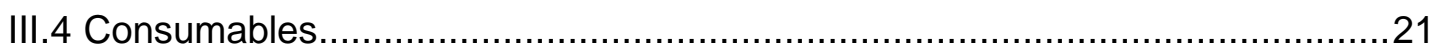

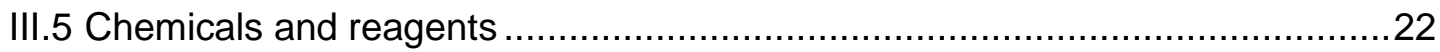

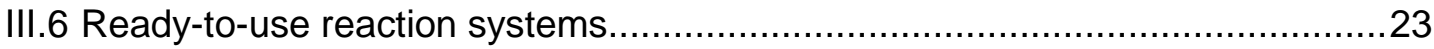

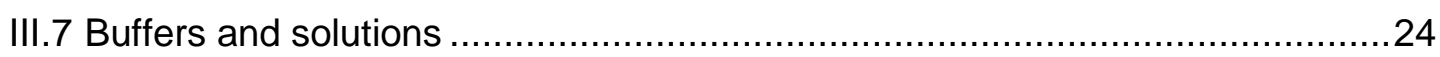

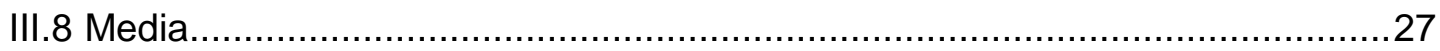

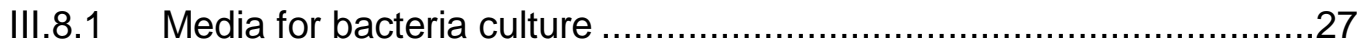

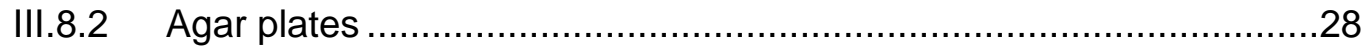

III.8.3 Media and reagents for cultivation of eukaryotic cell lines.................28

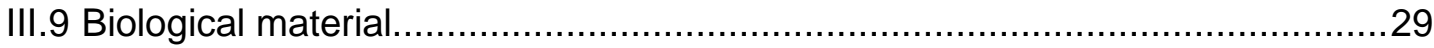

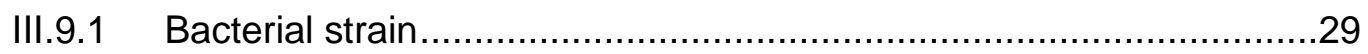

III.9.2 Eukaryotic cell lines ..............................................................29

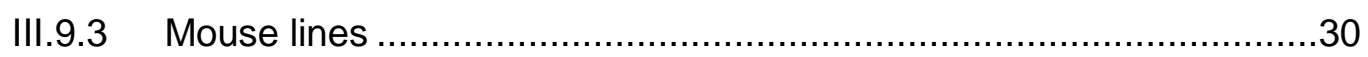

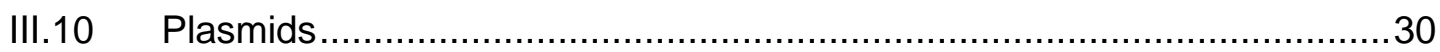

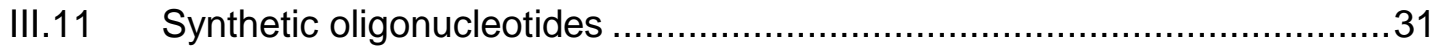

III.11.1 Synthetic DNA-oligonucleotides for qRT-PCR …............................31

III.11.2 Synthetic DNA-oligonucleotides for cloning …...............................33

III.11.3 Synthetic DNA-oligonucleotides for sequencing ............................33

III.11.4 Synthetic DNA-oligonucleotides for genotyping …...........................33

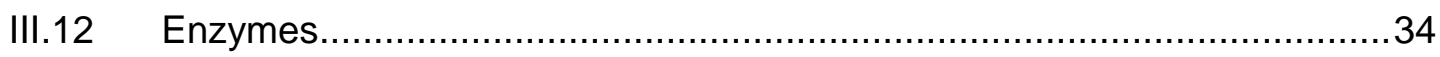

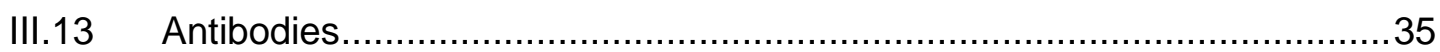




\section{Content}

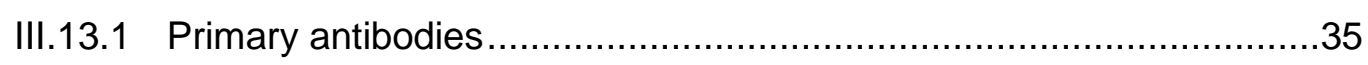

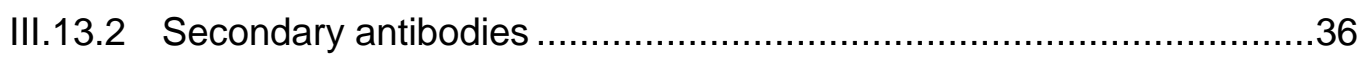

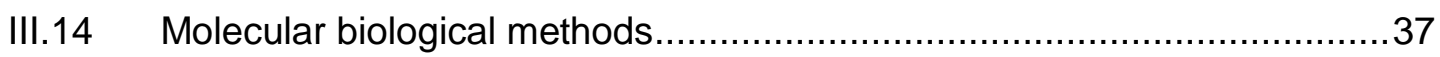

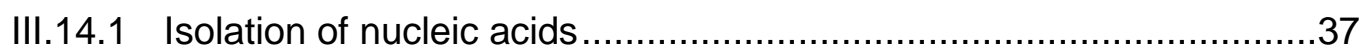

III.14.2 Polymerase chain reaction ......................................................... 40

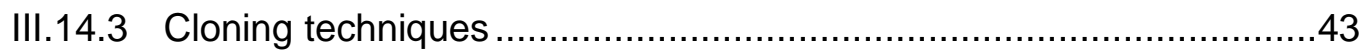

III.14.4 Cell biological techniques ........................................................4

III.14.4.1 Transformation of bacteria ......................................................47

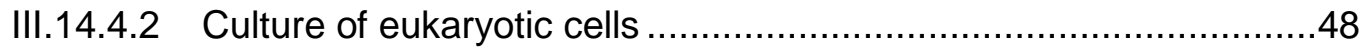

III.14.4.3 Isolation and cultivation of BMDMs .........................................48

III.14.4.4 Conditioned media (CM) ..................................................... 48

III.14.4.4.1 Generation and use of Wnt3a and Wnt5a CM ........................48

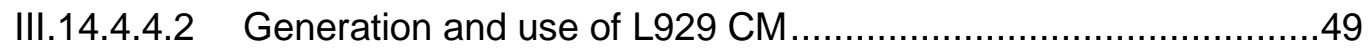

III.14.4.5 Concentration of Wif1-containing media.....................................49

III.14.4.6 Transfection of eukaryotic cells ..............................................49

III.14.4.7 Nucleofection of eukaryotic cells ..............................................50

III.14.4.8 Retroviral transduction of eukaryotic cells ..................................50

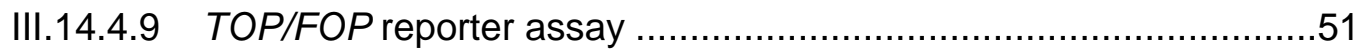

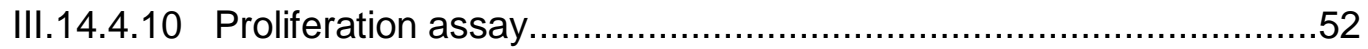

III.14.4.10.1 5-Bromo-2 -deoxyuridine assay ............................................52

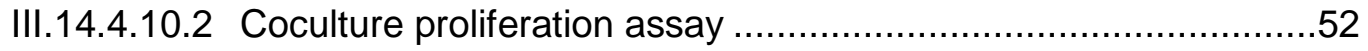

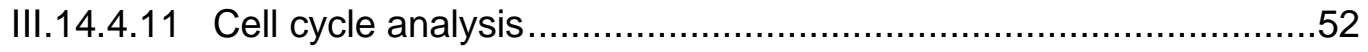

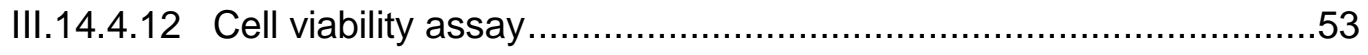

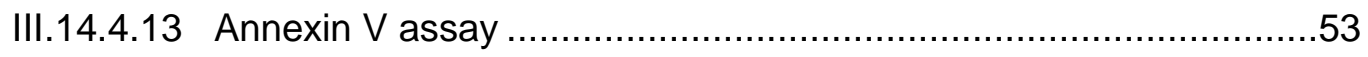

III.14.4.14 Transwell migration assay...................................................53

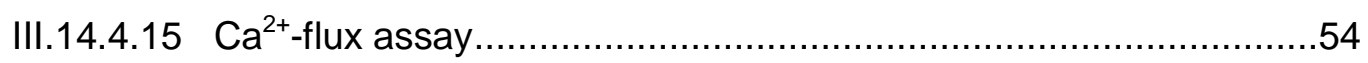

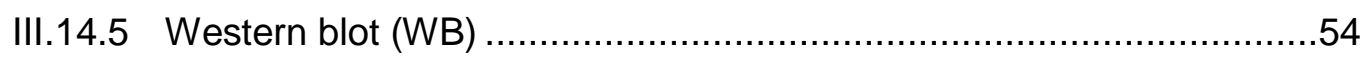

III.14.5.1 Isolation of proteins from cell culture ............................................54

III.14.5.2 Isolation of proteins from tissue samples....................................55

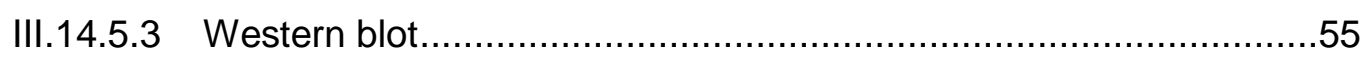

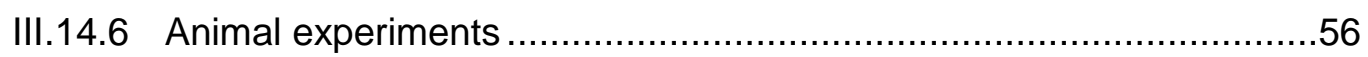

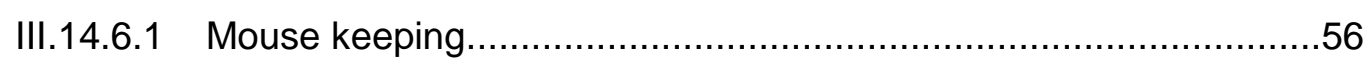

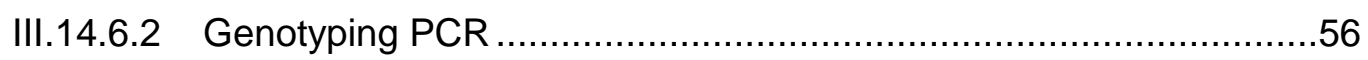

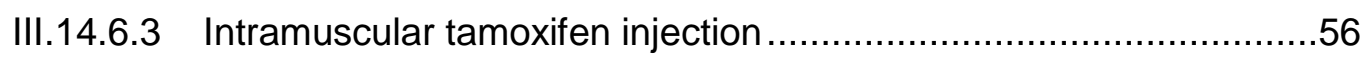

III.14.6.4 BCC mouse model and Wif1 knockout....................................57

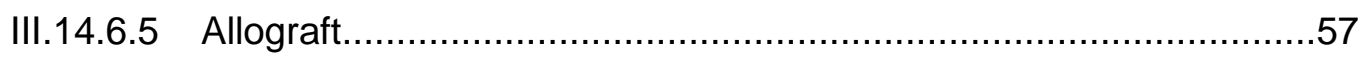

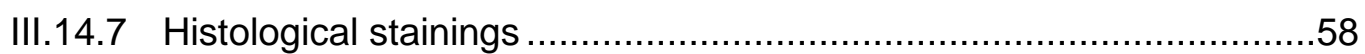




\section{Content}

III.14.7.1 Hematoxylin and eosin (H\&E) staining ....................................58

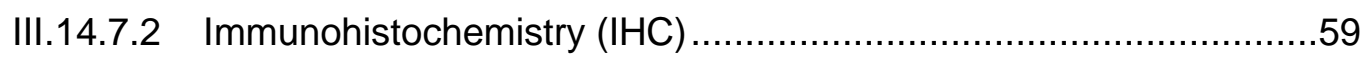

III.14.7.3 TdT-mediated dUTP-biotin nick end labeling (TUNEL) ..................59

III.14.7.4 Ladewig staining ...............................................................60

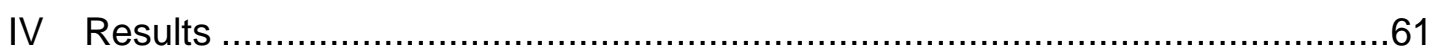

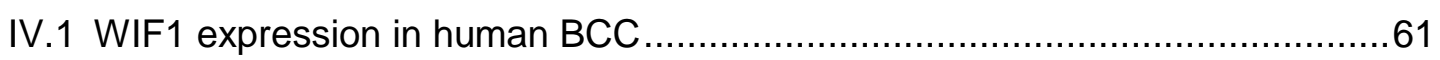

IV.2 Generation of a Wif1 expression vector .....................................................64

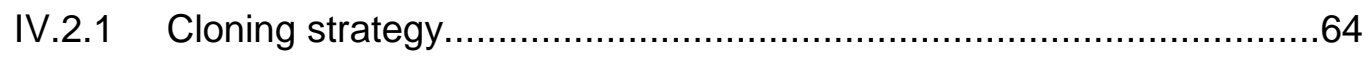

IV.2.2 Selection of stably transfected cells and verification of Wif1 expression 65

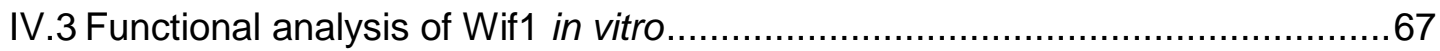

IV.3.1 TOP/FOP reporter assay for assessment of Wif1 activity .................67

IV.3.2 Analysis of $\mathrm{Hh}$ and Wnt pathway activity and $\mathrm{Ca}^{2+}$ measurement .....70 IV.4 Effect of Wif1 overexpression on various cellular processes of ASZ001 in vitro

IV.4.1 Effect of Wif1 on cell viability in vitro ..........................................

IV.4.2 Effect of Wif1 on apoptosis in vitro.................................................

IV.4.3 Effect of Wif1 on cell cycle regulation and proliferation in vitro...........74

IV.4.4 Effect of Wif1 on migration in vitro ................................................

IV.4.5 Effect of Wif1 on differentiation in vitro........................................78

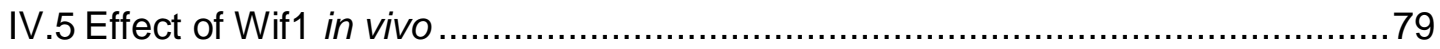

IV.5.1 Effect of Wif1 on Hh, Wnt and Akt signaling pathways in vivo............83

IV.5.2 Cellular response on Wif1 in vivo................................................ 87

IV.5.3 Summary of the effects of Wif1 overexpression .............................98

IV.6 Targeted disruption of Wif1 in murine BCC ................................................99

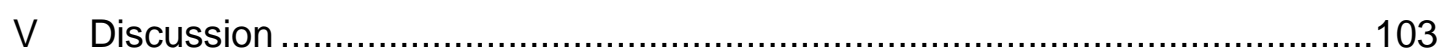

V.1 WIF1 in human tumors of the skin .................................................... 103

V.2 Wif1 overexpression in the BCC cell line ASZ001 .....................................105

V.2.1 Validation of Wif1 overexpression, secretion and functionality .........105

V.2.2 Effects of Wif1 overexpression on the BCC cell line ASZ001...........106

V.2.2.1 Effect of Wif1 on Wnt, Hh and EGFR signaling pathways ...............106

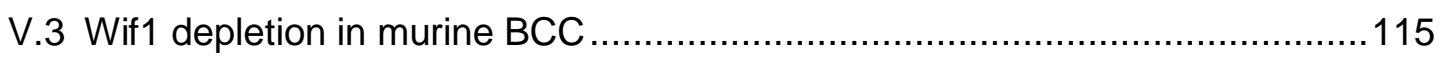

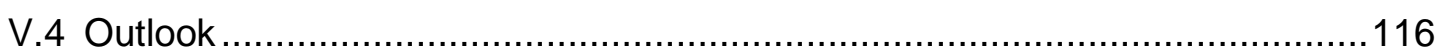

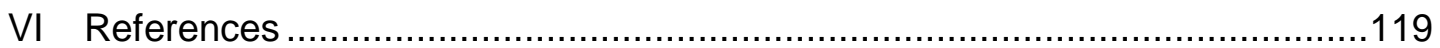

VII Abbreviations................................................................................. 132

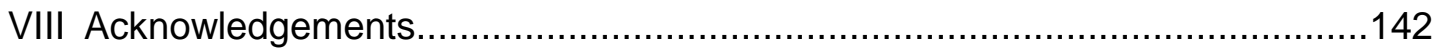

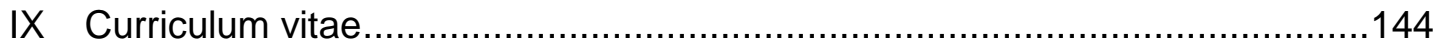




\section{List of Figures}

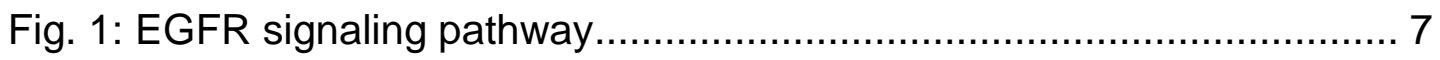

Fig. 2: Pathologically activated Hh signaling pathway................................ 9

Fig. 3: canonical Wnt signaling pathway ……............................................ 11

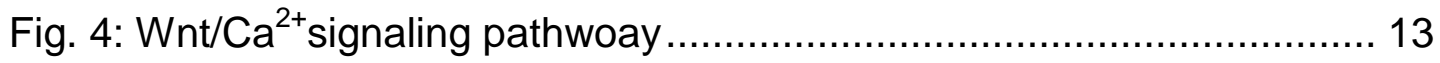

Fig. 5: Gel electrophoresis of amplified and purified Wif1 insert ................. 45

Fig. 6: Gel electrophoresis of hydrolyzed Wif1 insert and pMSCV vector

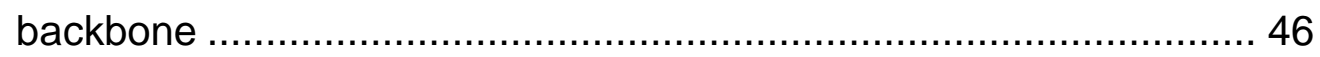

Fig. 7: Gel electrophoresis of restriction hydrolyzed pMSCV-Wif1 ............. 47

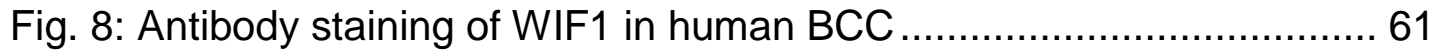

Fig. 9: Evaluation of WIF1 qRT-PCR of microdissected BCC subtypes, SCC and melanomas 63

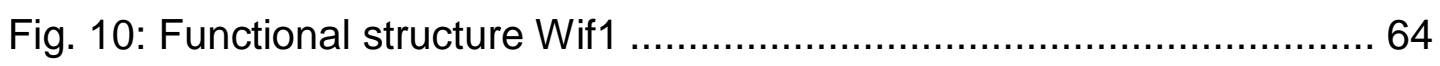

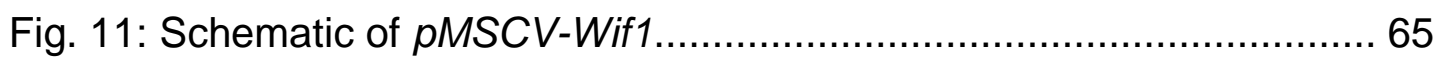

Fig. 12: PCR detecting Wif1 of $p M S C V$ and $p M S C V$-Wif1 transduced ASZ001 cells 66

Fig. 13: Western blot detecting Wif1 of $p M S C V$ and $p M S C V$-Wif1 transduced ASZ001 cells 66

Fig. 14: Western blot detecting Wif1 in supernatant and cellular extracts of ASZ-pMSCV and ASZ-Wif1

Fig. 15: TOP/FOP reporter assay of Wnt signaling in ASZ001 cells incubated with Wnt3a 68

Fig. 16: TOP/FOP reporter assay in HEK-293 cells incubated with Wnt3a and Wif1-concentrated supernatant ..................................................... 70

Fig. 17: qRT-PCR of Axin2 and Gli1 in ASZ-pMSCV and -Wif1 .................. 71

Fig. 18: $\mathrm{Ca}^{2+}$-influx assay of ASZ-pMSCV and -Wif1 ................................ 72

Fig. 19: WST-1 assay of ASZ-pMSCV and -Wif1 ................................... 73

Fig. 20: Annexin $V$ assay of uninduced and staurosporine induced ASZ-pMSCV and -Wif1 74

Fig. 21: Distribution of ASZ-pMSCV and-Wif1 in respective phases of cell cycle. 75

Fig. 22: BrdU assay of ASZ-pMSCV and-Wif1 76 


\section{List of Figures}

Fig. 23: Cell proliferation assay of ASZ-pMSCV and ASZ-Wif1 cocultured with $B 9, L$ cells, Wnt3A L cells, NIH/3T3, Wnt5a-NIH/3T3 or BMDMs . 77

Fig. 24: Transwell migration assay of ASZ-pMSCV and -Wif1 78

Fig. 25: qRT-PCR of Krt10 in ASZ-pMSCV and -Wif1 79

Fig. 26: Growth curve of ASZ-pMSCV and ASZ-Wif1 allografts in nude mice

Fig. 27: Tumor weight of ASZ-pMSCV and ASZ-Wif1 allografts after dissection

Fig. 28: H\&E stainings of ASZ-pMSCV and ASZ-Wif1 allografts 82

Fig. 29: Total sections of ASZ-pMSCV and ASZ-Wif1 allografts. 82

Fig. 30: Vital tumor area of ASZ-pMSCV and ASZ-Wif1 allografts. 83

Fig. 31: qRT-PCR of Gli1, Axin2 and c-Myc expression in ASZ-pMSCV and ASZ-Wif1 allografts

Fig. 32: Antibody staining of $\beta$-catenin in ASZ-pMSCV and ASZ-Wif1 allografts 84

Fig. 33: Western blot of pJNK and pCaMKII in ASZ-pMSCV and ASZ-Wif1 allografts 85

Fig. 34: Western blot of pAkt, Akt, pS6 and S6 in ASZ-pMSCV and ASZ-Wif1 allografts 86

Fig. 35: Western blot of pPKC in ASZ-pMSCV and ASZ-Wif1 . 86

Fig. 36: Western blot of pErk1 and 2 and Erk1 and 2 in ASZ-pMSCV and ASZ-Wif1 allografts

Fig. 37: qRT-PCRs of early and late keratinocyte differentiation markers in ASZ-pMSCV and ASZ-Wif1 allografts 88

Fig. 38: Proportion of keratinous tissue in ASZ-pMSCV and ASZ-Wif1 allografts 89

Fig. 39: qRT-PCRs of EMT markers in ASZ-pMSCV and ASZ-Wif1 allografts 90

Fig. 40: Number of CD34 positive blood vessels in ASZ-pMSCV and ASZ-Wif1 allografts 91

Fig. 41: Western blot of Caspase 3 in ASZ-pMSCV and ASZ-Wif1 allografts 92

Fig. 42: Nuclear p53 staining of ASZ-pMSCV and ASZ-Wif1 allografts. ...... 92

Fig. 43: TUNEL staining of ASZ-pMSCV and ASZ-Wif1 allografts.............. 93

Fig. 44: Ladewig staining of ASZ-pMSCV and ASZ-Wif1 allografts ..... 94 


\section{List of Figures}

Fig. 45: Collagen proportion in ASZ-pMSCV and ASZ-Wif1 allografts......... 95

Fig. 46: Western blot of Sdc-2 in ASZ-pMSCV and ASZ-Wif1 allografts...... 95

Fig. 47: Evaluation of Ki67 staining of ASZ-pMSCV and ASZ-Wif1 allografts 96

Fig. 48: Western blot of cyclin D1 in ASZ-pMSCV and ASZ-Wif1 allografts. 97 Fig. 49: antibody staining of F4/80 in ASZ-pMSCV and ASZ-Wif1 allografts 97 Fig. 50: Antibody staining of aSma in ASZ-pMSCV and ASZ-Wif1 allografts 98

Fig. 51: Antibody staining of Wif1 in murine BCC 99

Fig. 52: Western blot of Wif1 in murine BCC 100

Fig. 53: Planimetric measurement of tumor size in Ptch $^{\text {flox/flox }} \mathrm{CreERT2}^{T /-}$ and Ptch $^{\text {flox/flox }}$ CreERT2 ${ }^{T /-}$ Wif1 $1^{-/}$skin samples $45 \mathrm{~d}$ after induction ....... 101

Fig. 54: Planimetric measurement of tumor size in Ptch $^{\text {flox/flox }} \mathrm{CreERT2}^{T /-}$ and Ptch $^{\text {flox/flox }}$ CreERT2 ${ }^{T /-}$ Wif1 $^{-/}$skin samples 90d after induction ....... 101

Fig. 55: Evaluation of anti-Ki67 antibody staining in Ptch $^{\text {flox/flox }} \mathrm{CreERT2}^{T / \text { - }}$ and Ptch ${ }^{\text {flox/flox }}$ CreERT2 ${ }^{T /-}$ Wif1 ${ }^{-/-}$skin samples $45 \mathrm{~d}$ and $90 \mathrm{~d}$ after induction 102

Fig. 56: Putative autocrine and paracrine signaling mechanism in ASZ-Wif1 allografts 


\section{List of Tables}

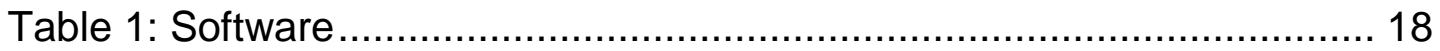

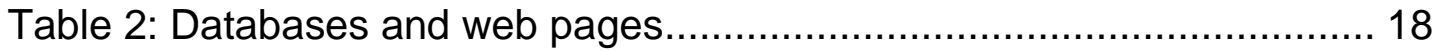

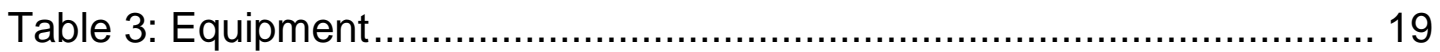

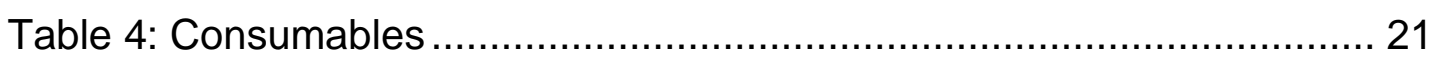

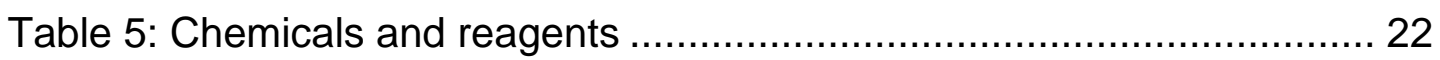

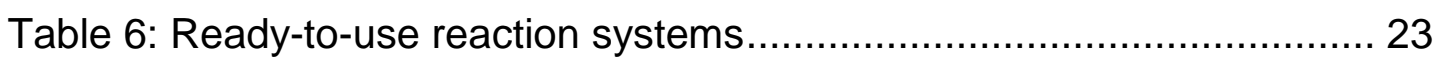

Table 7: Cell culture media and supplements for eukaryotic cell lines ......... 28

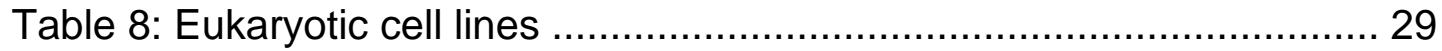

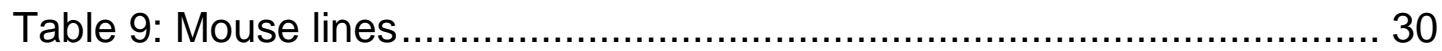

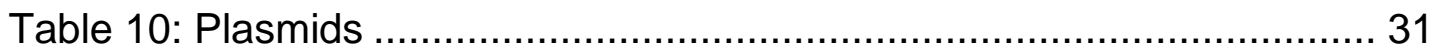

Table 11: Oligonucleotides for qRT-PCR analyses ................................... 32

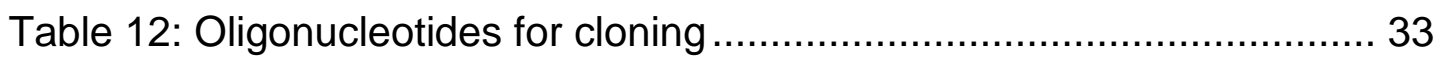

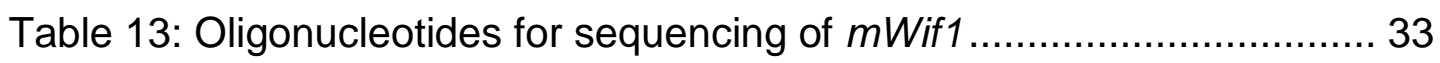

Table 14: Oligonucleotides for genotyping ................................................ 33

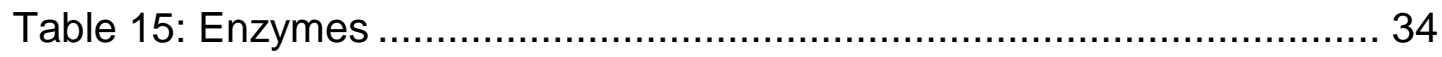

Table 16: Primary antibodies for immunohistochemistry........................... 35

Table 17: Primary antibodies for Western blot ............................................. 35

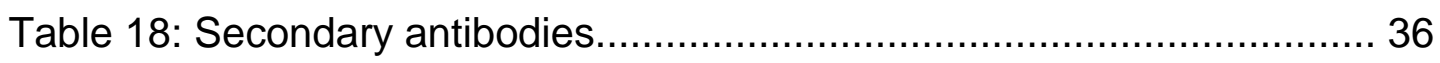

Table 19: Scheme of plasmid combinations for nucleofection ..................... 50 


\section{Summary}

Basal cell carcinoma (BCC) belongs to the group of non-melanoma skin cancers and is the most commonly diagnosed cancer in fair-skinned individuals. In the majority of BCC the tumor suppressor gene patched1 $(P T C H)$ is mutated resulting in aberrant hedgehog $(\mathrm{HH})$ signaling. Analysis of human and murine BCC shows that the putative tumor suppressor Wnt inhibitory factor 1 (Wif1) is highly expressed by this tumor entity. However, malignant forms of skin cancer, i.e. squamous cell carcinomas and melanomas, also express Wif1. Thus, the objective of this thesis was to analyze the role of Wif1 in BCC by Wif1 overexpression in the BCC cell line ASZ001 and by Wif1 depletion in the BCC mouse model Ptch $^{\text {floxfflox }}$ CreERT2 ${ }^{\text {T/ }}$.

Indeed, tumor-intrinsic Wif1 overexpression in ASZ001 significantly inhibits tumor growth in nude mice. The Wif1-mediated tumor suppression was not due to diminished vascularization or alterations in canonical Wnt, $\mathrm{Hh}$ or PI3K/Akt signaling activity. It also was not due to induction of differentiation or apoptosis. However, we found that Wif1-expressing tumors were characterized by a decrease in Ki67 positive cells which was accompanied by phosphorylation of PKC and Erk1 along with moderately increased deposition of collagens. Vice versa, BCC growth is enhanced in Ptch-knockout mice on a Wif1-deficient background due to an increase in proliferation. Together, the data suggest that Wif1 is both necessary and sufficient to restrict BCC growth and may be one of the factors that are responsible for the very low metastatic potential of this tumor entity. 
II Introduction

\section{II.1 Basal cell carcinoma (BCC)}

BCC belongs to the group of non-melanoma skin cancers and is the most commonly diagnosed cancer in fair-skinned individuals. As it is not reported to cancer registries, the precise incidence of BCC is unknown. However, it is estimated that BCC account for nearly half of all cancers in the United States of America (USA). In Europe, the incidence of BCC ranges from 44.6 to 128 per 100,000 inhabitants (Rubin et al. 2005; Trakatelli et al. 2007)(American Cancer Society 2015). The average lifetime risk for fair-skinned individuals to develop a BCC is approximately $30 \%$ (Abdulla et al. 2005). Thus, BCC is the most common tumor in humans.

In contrast to other skin tumors such as squamous cell carcinoma (SCC) and melanoma, BCC are semi-malignant tumors. They have a good overall prognosis, with a high chance of cure when diagnosed early (Robinson and Dahiya 2003). This is due to the fact that BCC have a low metastatic potential as metastasis occurs in only $0.0028-0.55 \%$ of all patients (Soleymani et al. 2008). In addition, approximately $20 \%$ of BCC show signs of spontaneous regression (Curson and Weedon 1979). However, BCC can cause substantial morbidity due to its multiplicity, local recurrence and tissue invasion and destruction (Basset-Seguin et al. 2015).

The clinical presentation of BCC is diverse. Consequently, BCC can be classified histopathologically as indolent-growth or aggressive-growth subsets. The indolent-growth variants comprise superficial and nodular BCC subtypes with nodular $\mathrm{BCC}$ being the most common $\mathrm{BCC}$ subtype (Crowson 2006). Aggressive-growth tumors include infiltrative BCC, metatypical BCC and morpheaform or sclerosing BCC.

BCC can also be classified according to their differentiation status. This includes keratotic, infundibulocystic, follicular, pleomorphic BCC, BCC with eccrine differentiation, BCC with sebaceous differentiation, the fibroepithelioma of Pinkus and BCC with myoepithelial differentiation (Crowson 2006). 
BCC most frequently occur at sun-exposed sites of the skin (Nakayama et al. 2011) and several epidemiologic studies indicate that exposure to sun light is the main risk factor for $\mathrm{BCC}$ formation. Other risk factors comprise fair skin, age, immune suppression and exposure to arsenic (Boonchai et al. 2000; Dessinioti et al. 2010).

Although the vast majority of BCC occur sporadically, a rare heritable disorder exists, that predisposes patients to the development of multiple BCC during their lifetime. This syndrome is known as basal-cell nevus syndrome (BCNS), or Gorlin-Goltz syndrome or nevoid basal-cell carcinoma syndrome. By genetic linkage analysis of relatives with BCNS, the mutation bearing locus was mapped to human chromosome 9q22 and then to the patched 1 (PTCH) gene (Hahn et al. 1996; Johnson et al. 1996). Indeed, mutations of PTCH are responsible for BCC development in BCNS patients. Upon this discovery, $P T C H$ was shown to be mutated in about $90 \%$ of all spontaneous BCC. Other BCC associated mutations are activating mutations in smoothened (SMO) (Lam et al. 1999), mutations in suppressor of fused homolog (SUFU) and in tumor protein p53 (TP53) (Reifenberger et al. 2005). BCC also develop in Ptch mutant mice comprising the Ptch ${ }^{\text {neo67/+ }}$ (Mancuso et al. 2004) and the Ptch ${ }^{\text {floxflox }}$ (Zibat et al. 2009; Nitzki et al. 2010) mouse models, in a mouse model expressing a constitutively active Smo mutant (SmoM2) (Xie et al. 1998) and in a mouse model overexpressing sonic hedgehog (Shh) (Oro et al. 1997). These mouse models are valuable tools to investigate the molecular mechanisms of BCC pathogenesis.

The cellular origin of BCC is still debated. However, analysis of Ptch knockout or transgenic SmoM2 mouse models revealed that BCC can originate from progenitor cells of the interfollicular epidermis and the upper infundibulum of the hair shaft (Youssef et al. 2010) and from hair follicle stem cells (Nitzki et al. 2010; Peterson et al. 2015) caused by aberrantly activated Hedgehog $(\mathrm{Hh})$ signaling.

BCC treatment comprises surgical or non-surgical techniques depending on several tumor- and patient-related factors. The best results are achieved upon surgery including Mohs micrographic surgery, La Galette technique, 
conventional surgery with tumor-adapted margins of safety, curettage, electrodesiccation, and cryosurgery (Goppner and Leverkus 2011). Non-surgical treatment options comprise radiotherapy, photodynamic therapy, and topical application of imiquimod and 5-fluorouracil. For advanced or metastatic BCC the Food and Drug Administration (FDA) approved the Smo inhibitor vismodegib (trade name Erivedge ${ }^{\circledR}$ ) that can be either applied systemically or topically (Basset-Seguin et al. 2015). In particular, patients with multiple comorbidities and inoperable tumors benefit from these non-surgical techniques. However, compared to surgery nonsurgical treatments can result in increased recurrence rates.

Due to its high and rising incidence and its increasing occurrence in young people $\mathrm{BCC}$ are becoming more and more problematic for the health care system. Non-melanoma skin cancer (i.e. BCC and SCC) account for $9 \%$ of the total costs of all cancers in 2000-2001 in Australia (Staples et al. 2006). Better therapeutic approaches may help to reduce these costs. This, however, necessitates a better knowledge about molecular events in BCC growth, progression or regression. Particularly factors and mechanisms that keep BCC a slowly growing tumor entity could enable us to employ these findings as prospective treatment options.

\section{II.2 Signaling pathways in BCC}

Multiple pathways are known to be misregulated in BCC. Among these are the $\mathrm{Hh}$ and Wnt signaling pathway (see below). Moreover, components of pathways regulating cell cycle progression and controlling apoptosis are frequently found to be mutated (TP53) or misregulated (platelet derived growth factor receptor- $\alpha$ (PDGFRa) and B cell leukemia/lymphoma 2 (BCL2)) in BCC (Xie et al. 2001; Vidal et al. 2004; Reifenberger et al. 2005).

Furthermore, epidermal growth factor receptor (EGFR) signaling plays an important role in BCC. Receptors of this pathway, ErbB1, 2 and 3 were shown to be expressed in BCC (Krahn et al. 2001; Schnidar et al. 2009) and epidermal-specific deletion or irreversible inhibition of EGFR inhibits BCC 
growth (Eberl et al. 2012). Downstream of activated EGFR several signaling cascades including mitogen-activated protein kinase (MAPK) and phosphatidylinositol-4,5-bisphosphate 3-kinase/v-akt murine thymoma viral oncogene homolog (PI3K/Akt) signaling have been reported to be involved in BCC growth (Schnidar et al. 2009; Hafner et al. 2010). In particular, the inhibition of the Mapk kinase 1/2 (Mek1/2) was shown to suppress the proliferation of the BCC cell line ASZ001 (Xie et al. 2001).

As EGFR signaling is also relevant in this thesis a more detailed summary of this pathway is given. EGFR signaling is involved in the regulation of a variety of cellular responses including proliferation, differentiation, migration and apoptosis (Yarden and Sliwkowski 2001; Chong and Janne 2013) and has a crucial role in the initiation and maintenance of several solid tumors (Arteaga and Engelman 2014). In the extracellular space ligands containing epidermal growth factor (EGF)-like domains can bind to the v-erb-b2 erythroblastic leukemia viral oncogene homolog (ErbB) receptors (Yarden and Sliwkowski 2001). ErbB family members are receptor tyrosine kinases (RYKs) and comprise the epidermal growth factor receptor ErbB1 (EGFR, also HER1), ErbB2 (also HER2 or Neu), ErbB3, and ErbB4 (Yarden and Sliwkowski 2001). Upon binding of EGF-like growth factors to the extracellular domain, the ErbB receptors form homo- and heterodimers resulting in auto- or transphosphorylation of the cytoplasmic domains. The phosphorylated domains serve as binding sites for various proteins involved in the regulation of multiple intracellular signaling cascades (Yarden and Pines 2012). Which signaling cascade is activated depends on the respective receptor dimers, as all ErbB family members have distinct biochemical properties and interaction partners (see Fig. 1) (Nyati et al. 2006; Yarden and Pines 2012).

First, EGFR phosphorylation can induce the activation of signal transducer and activator of transcription (STAT) by Janus kinase (JAK). Activated STAT in turn translocates into the nucleus and directly regulates gene expression crucial for cell survival, proliferation, transformation and oncogenesis (Bowman et al. 2000). 
Second, EGFR activates PI3K that phosphorylates $\mathrm{PIP}_{2}$ to form phosphatidylinositol-3,4,5-triphosphate $\left(\mathrm{PIP}_{3}\right)$, which then activates Akt by phosphorylation. Phosphorylated Akt has several effects, both in the cytoplasm and in the nucleus, which include the inhibition of proapoptotic factors such as BAD (BCL2 antagonist of cell death), pro-caspase 9 and the Forkhead (FKHR) family of transcription factors (FOXO). Moreover, Aktmediated activation of mammalian target of rapamycin (mTOR) is involved in the regulation of cell proliferation by controlling the activity of ribosomal protein S6 (S6) (Wendel et al. 2004; Ruvinsky and Meyuhas 2006; Hemmings and Restuccia 2015).

Third, activation of small GTPase rat sarcoma virus oncogene homolog (Ras) by phosphorylation is mediated by son of sevenless (SOS) triggering a MAPK signaling cascade. Activated Ras binds to Raf (MAP3K), which in turn triggers the phosphorylation of MEK $1 / 2$ and MAPK3/1 (also named extracellular signal-regulated kinases (ERK) $1 / 2$ or p44/p42). Phosphorylated ERK1/2 translocates into the nucleus and activates various transcription factors such as ELK1 (McCubrey et al. 2012).

Fourth, PLC binds to phosphorylated EGFR inducing its activity. Subsequently, PLC hydrolyses phosphatidylinositol-4,5-bisphosphate $\left(\mathrm{PIP}_{2}\right)$ to 1,2 diacylglycerol (DAG) and inositol 1,4,5-triphosphate $\left(\mathrm{IP}_{3}\right)$. DAG mediates the activation of protein kinase $\mathrm{C}(\mathrm{PKC})$ regulating cell-cycle progression, transformation, differentiation and apoptosis (Oliva et al. 2005). 


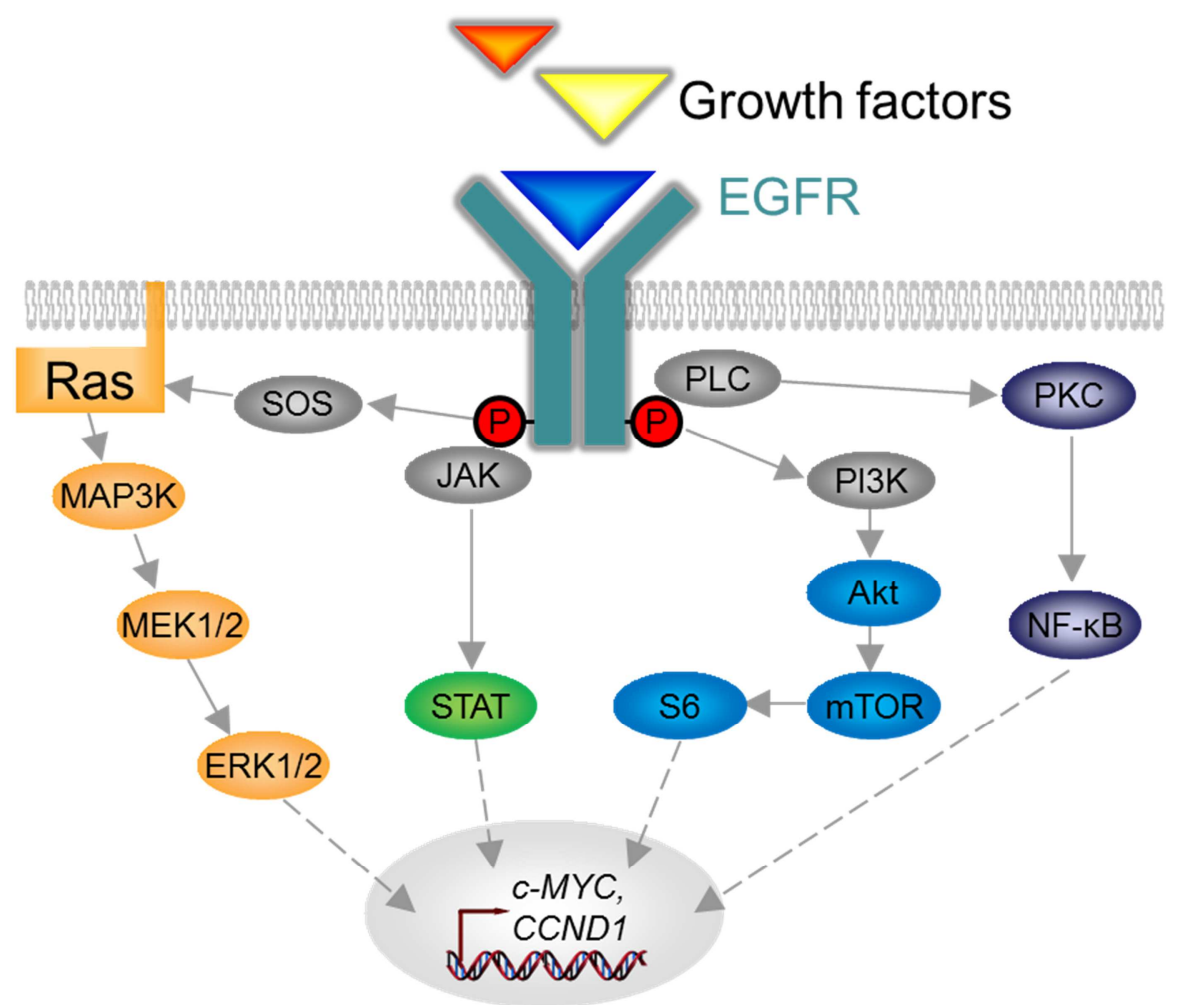

Fig. 1: EGFR signaling pathway

The shown signaling cascade does not include all the known components of a given pathway, and cross-talks are not shown for clarity. Activated EGFR signaling can result in the induction of different pathways leading to the activation of ERK, STAT, Akt, S6 and/or PKC. Modified from: (Nyati et al. 2006).

\section{II.3 Hh signaling}

The Hh signaling pathway is highly conserved between the species and its signaling is required for tissue homeostasis and regeneration (Beachy et al. 2004). In brief, $\mathrm{Hh}$ is a morphogen and three mammalian homologues of the Drosophila segment polarity gene hedgehog exist. These are sonic (Shh), indian (Ihh) and desert (Dhh) hedgehog, all of which are all secreted proteins. Shh is the most broadly expressed $\mathrm{Hh}$ homologue. In vertebrate development Shh is mainly required for polarization and pattern formation of the limb bud and of the neural tube. Inh regulates bone and cartilage development and Dhh is essential for germ cell development in the testis and peripheral nerve sheath formation (Petrova and Joyner 2014). Hh signaling requires the autoproteolytic cleavage of the $\mathrm{Hh}$ precursor into an $\mathrm{N}$-terminal (Hh-N) and a C-terminal (Hh-C) moiety. After several post-translational modifications including an addition of a cholesterol moiety at the C-terminus 
and palmitoylation at the $\mathrm{N}$-terminus of $\mathrm{Hh}-\mathrm{N}$, it is secreted by means of the membrane transporter protein dispatched (Disp) (Burke et al. 1999; Ma et al. 2002; Beachy et al. 2010).

In the extra cellular space the distribution of $\mathrm{Hh}$ is dependent on heparan sulfate proteoglycans (HSPGs) (Chang et al. 2011), where it can bind to the two extracellular loops of the 12-pass transmembrane receptor Ptch (Briscoe et al. 2001). The association of $\mathrm{Hh}$ to Ptch is increased by the transmembrane proteins cysteine dioxygenase 1, cytosolic (Cdo1) and biregional cell adhesion molecule-related/down-regulated by oncogenes (Cdon) binding protein (Boc) (Yao et al. 2006). Ptch is a negative regulator of the Hh signaling pathway (Epstein 2008); (Pasca di Magliano and Hebrok 2003). In the absence of $\mathrm{Hh}$ ligand, Ptch inhibits the activation of the Gprotein coupled receptor Smo, in turn keeping glioma-associated oncogene family member (Gli) transcription factors inactive in the cytoplasm. When $\mathrm{Hh}$ is present, Ptch becomes internalized into the cell, resulting in suspension of Smo inhibition. Subsequently, Smo facilitates the translocation of the activator forms of Gli transcription factors into the nucleus where they induce the expression of $\mathrm{Hh}$ target genes (see Fig. 2) by binding to the conserved Gli binding site 5'-GACCACCCA-3' (Hallikas et al. 2006). The Gli protein family consists of 3 distinct proteins (Gli1, 2 and 3). Gli2 and Gli3 are bifunctional transcription factors facilitating either transcriptional repression or activation. When Hh signaling is inactive Gli2 and Gli3 are phosphorylated and subsequently proteolytically cleaved to generate the repressor forms (Gli2R and Gli3R, respectively). When Hh is present, the activation of Smo results in the transport of uncleaved, activated Gli2 and Gli3 proteins from the cilia to the nucleus. Gli1, which is a downstream target gene of active $\mathrm{Hh}$ signaling but not immediately involved in signal transduction, is a strong potentiator of the signaling cascade (Roberg-Larsen et al. 2014).

When Ptch is mutated, the resulting protein is incapable of inhibiting Smo. This results in pathological, constitutive active $\mathrm{Hh}$ signaling, ultimately leading to cancer formation such as BCC in both humans and mice (see Fig. 2) (Athar et al. 2006). 


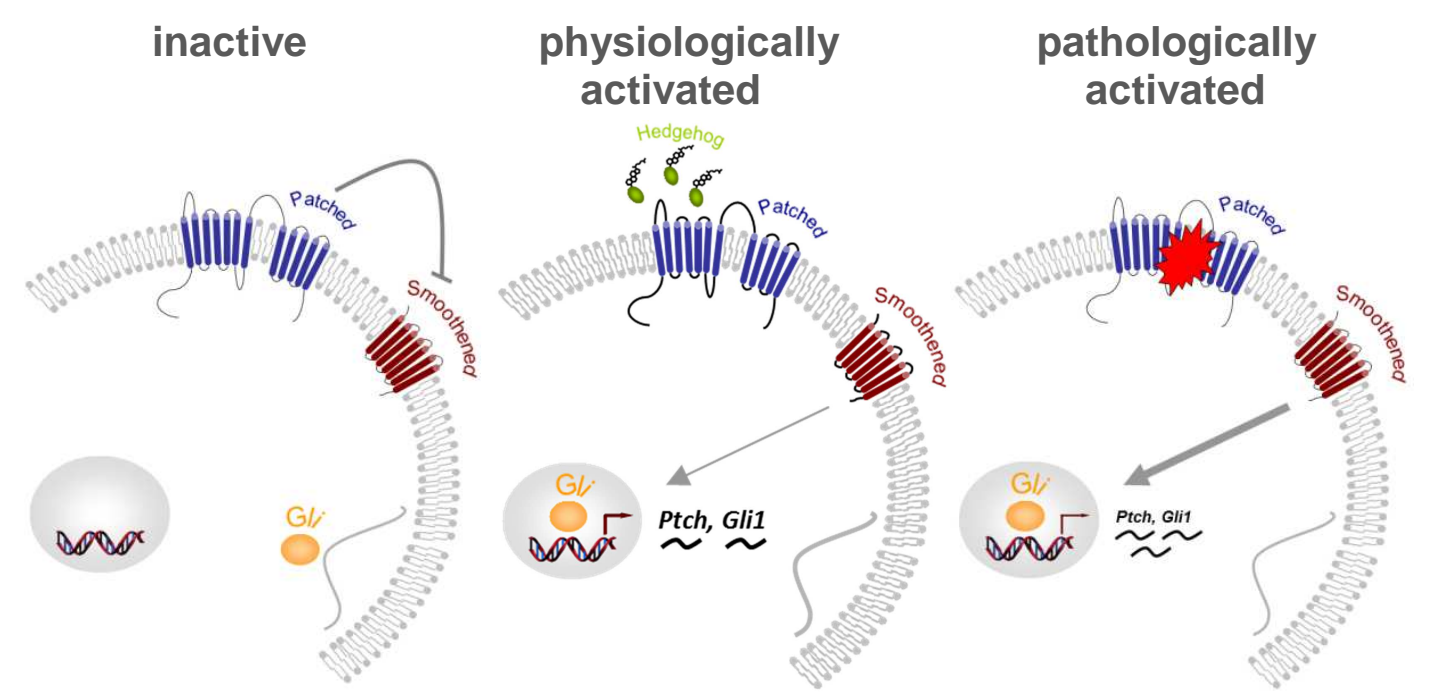

Fig. 2: Pathologically activated Hh signaling pathway

Left panel: inactive pathway, Ptch inhibits Smo and Gli transcription factors remain inactive in cytoplasm; middle panel: $\mathrm{Hh}$ binds to its receptor Ptch leading to a translocation of Gli transcription factors into the nucleus; right panel: mutated Ptch cannot inhibit Smo and Gli is constitutively triggering target gene expression. Modified from: (Nitzki 2008).

\section{II.4 Wnt signaling}

The wingless-type MMTV integration site family member (Wnt) signaling is a central pathway in development, tissue regeneration and homeostasis. Wnts are secreted proteins that act as morphogens in the extracellular space. For secretion they require the activity of the palmitoyltransferase porcupine (Porcn) adding a palmitate modification to the premature Wnt proteins. This lipid modification leads to the binding of the 7-transmembrane protein wntless (WIs) conveying Wnt to the plasma membrane for secretion (Banziger et al. 2006). At the target cell Wnt ligands can induce the activity of $\beta$-catenindependent Wnt signaling (canonical) or $\beta$-catenin-independent (noncanonical) signaling. The non-canonical signaling comprises at least the Wnt/planar cell polarity (PCP) and $\mathrm{Wnt} / \mathrm{Ca}^{2+}$ signaling.

In the extracellular space Wnt signaling can be blocked at the receptor level by dickkopf (Dkk) binding to the single-span transmembrane receptor LDL receptor-related protein $5 / 6$ (Lrp5/6) or by direct binding and sequestration of Wnts either by the secreted frizzled-related protein (Sfrp) or by Wnt inhibitory factor 1 (Wif1) (Malinauskas and Jones 2014). 
When canonical Wnt signaling is inactive, $\beta$-catenin is continuously phosphorylated by the serine/threonine kinases glycogen synthase kinase 3 (Gsk3) and casein kinase 1 (Ck1) as part of the destruction complex (composed of Gsk3, Ck1, dishevelled (Dvl), Axin1, adenomatosis polyposis coli (Apc) and beta-transducin repeat containing protein ( $\beta$-TrCP)) (see Fig. 3). Phosphorylated $\beta$-catenin is subsequently ubiquitinated by $\beta-\operatorname{TrCP}$, a component of the E3 ubiquitin ligase complex, and degraded by the proteasome (Clevers and Nusse 2012). The absence of $\beta$-catenin allows the T-cell factor/lymphoid enhancer factor (Tcf/Lef) transcription factors, in particular Tcf3 and Tcf4 (Liu et al. 2005), to interact with transducin-like enhancer of split, homolog of Drosophila E (spl) (Tle, or groucho). The binding of Tle prevents Wnt target gene transcription (Cadigan and Waterman 2012). When canonical Wnt signaling is active, Wnt ligands bind with the palmitate group to the cysteine-rich domain of their receptor cognate frizzled (Fzd) receptor. Moreover, Wnt ligands bind the Lrp5/6 co-receptor, inducing it to form a complex with Fzd. This results in a conformational change of the receptors inducing the phosphorylation of Lrp5/6. This recruits Axin1 as part of the intact destruction complex leading to the dissociation of $\beta$-TrCP. Thus, phosphorylated $\beta$-catenin is no longer ubiquitinated and consequently not degraded. This leads to a saturation with phosphorylated $\beta$-catenin at the destruction complex and inhibition of the latter ( $\mathrm{Li}$ et al. 2012). Only $\beta$-catenin that is newly synthesized after initiation of the Wnt signal is signaling competent (Staal et al. 2002). In its active stage $\beta$-catenin remains unphosphorylated and accumulates in the cytoplasm resulting in its translocation into the nucleus. In the nucleus active $\beta$-catenin can bind to Tcf/Lef transcription factors, in particular Tcf1 and Lef1 (Behrens et al. 1996; Molenaar et al. 1996; Liu et al. 2005). Tcf/Lef transcription factors can directly bind to DNA bearing the motif 5 -CCTTTGATCTT-3` (van de Wetering et al. 1997) which is also employed in the TOP/FOP reporter system (Korinek et al. 1997). When $\beta$-catenin binds Tcf/Lef transcriptional activators in a complex containing CREB-binding protein (Cbp), B cell CLL/lymphoma 9 (Bcl9) and pygopus (Pygo) it drives Wnt target genes expression in a tissue- and developmental stage-specific manner. Axin2 is considered as a general transcriptional target gene of active $\mathrm{Wnt} / \beta$-catenin signaling and thus serves 
an indicator of an active signaling pathway (Lustig et al. 2002). In contrast to the proposed model of an intact destruction complex and the dissociation of $\beta$-TrCP described above, it is also discussed that the destruction complex is resolved into its compounds as a result of recruitment of Axin to the Fzd receptor. Which model turns out to hold true is an ongoing debate.

inactive
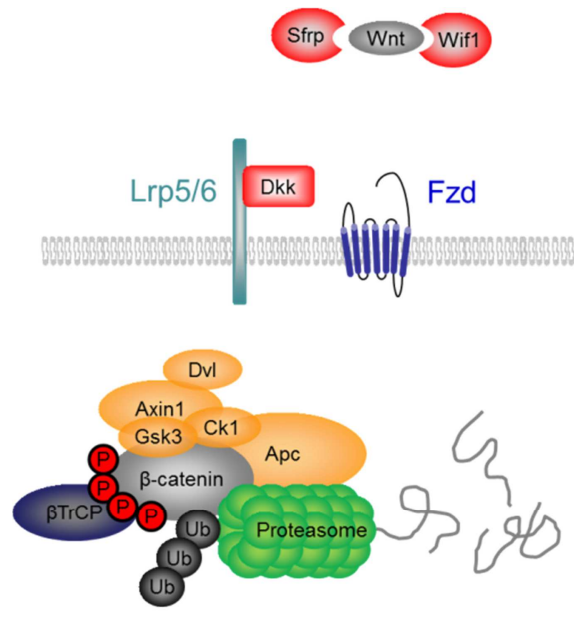

0000000 activated

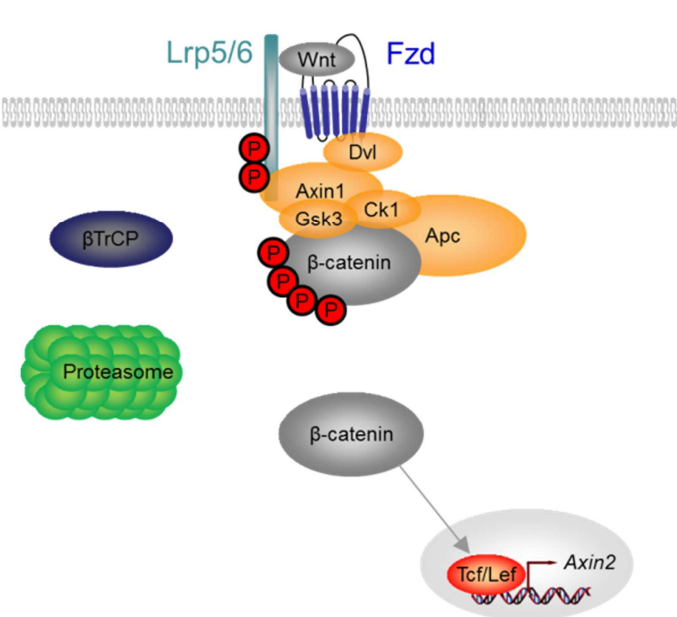

Fig. 3: canonical Wnt signaling pathway

Left panel: inactive pathway; proteasome cleaves ubiquitinated $\beta$-catenin preventing target gene expression. Right panel: active pathway; Conformational change of the activated receptors result in the dissociation of $\beta \operatorname{TrCP}$ abrogating ubiquitination of $\beta$-catenin and thus proteasomal degradation; newly synthesized $\beta$-catenin accumulates and translocates into the nucleus triggering target gene expression. Modified from: (Clevers and Nusse 2012).

In contrast to canonical Wnt signaling, the Wnt/PCP pathway is independent from $\beta$-catenin. It controls the cell shape, directional migration, asymmetric cell division, and cellular orientation which is required for normal development and function of complex tissues (Kaucka et al. 2015). Wnt/PCP signaling is activated by the binding of Wnts to Fzd receptor and the coreceptors receptor tyrosine kinase-like orphan receptor 1 and 2 (Ror1/2), the Ryk, or the protein tyrosine kinase 7 (Ptk7) (Green et al. 2014). At the cell surface PCP signaling can be blocked by vang-like (Vangl, or strabismus). The cytoplasmic tail of Vangl binds and recruits prickle $(\mathrm{Pk})$ to the plasma membrane, where Prickle binds and thereby antagonizes the recruitment of Dvl to Fzd resulting in an inhibition of Wnt/PCP signaling (Wang 2009). Activation of the pathway by Wnt ligands results in the recruitment of 
cytoplasmic Dvl to the Fzd receptor. When the pathway is active, ankyrin repeat domain (Ankrd, or diego) binds to Dvl and prevents Pk from binding and inhibiting Dvl (Devenport 2014). At the level of Dvl, two parallel pathways result in the activation of the GTPases Rho and Rac (Habas et al. 2003). The first activates dishevelled associated activator of morphogenesis 1 (Daam1) resulting in the activation of the Rho-associated coiled-coil containing protein kinase (Rock) inducing cytoskeletal reorganization (Kim and Han 2005). Furthermore, Daam1 is a formin-homology protein and a direct nucleator of linear actin filaments (Watanabe and Higashida 2004). The second pathway activates Rac, which in turn stimulates Mapk8 (JNK) activity modulating actin cytoskeleton (Habas et al. 2003).

The second $\beta$-catenin independent Wnt pathway is $\mathrm{Wnt} / \mathrm{Ca}^{2+}$ signaling (see Fig. 4). This pathway is activated upon binding of specific Wnt ligands to Fzd receptor leading to the activation of phospholipase C (PLC). Activated PLC cleaves membrane bound phospholipid $\mathrm{PIP}_{2}$ in $\mathrm{IP}_{3}$ and $\mathrm{DAG}$. $\mathrm{IP}_{3}$ triggers $\mathrm{Ca}^{2+}$ influx from the endoplasmic reticulum (ER), which activates several effector proteins including calcium/calmodulin-dependent protein kinase II (CaMKII), PKC and calcineurin ( $\mathrm{Cn}$ ). These kinases regulate and activate a multitude of target proteins including nuclear factor of kappa light polypeptide gene enhancer in B cells (Nf-KB), CAMP responsive element binding protein (Creb), Erk and nuclear factor associated with $\mathrm{T}$ cells (Nfat) (Sheldahl et al. 1999; Kuhl et al. 2000; Hogan et al. 2003) that in turn drive the expression various target genes regulating cell survival and proliferation. 


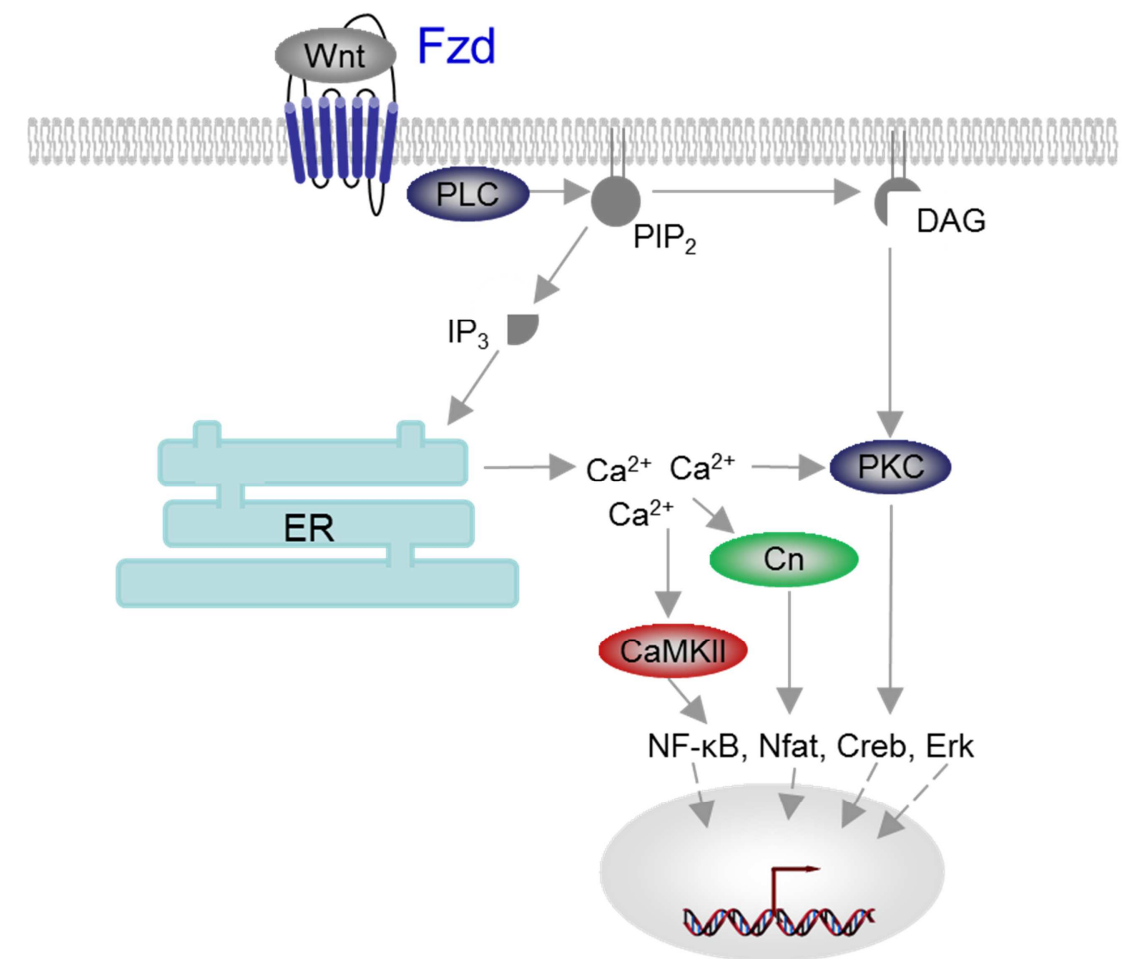

Fig. 4: Wnt/Ca ${ }^{2+}$ signaling pathway

Wnt binding to the receptor Fzd triggers the activation of PLC. This leads to the cleavage of $\mathrm{PIP}_{2}$ in $\mathrm{DAG}$ and $\mathrm{IP}_{3}$. $\mathrm{IP}_{3}$ induces an efflux of $\mathrm{Ca}^{2+}$ from the endoplasmic reticulum (ER) into the cytoplasm. Elevated cytoplasmic $\mathrm{Ca}^{2+}$ levels and DAG induce the activation of CaMKII, $\mathrm{Cn}$ and PKC triggering target gene expression by various effector proteins. Modified from: (Lories et al. 2013).

Although the canonical and non-canonical pathways are here separately described, they influence and inhibit each other at various interfaces (Weidinger and Moon 2003). In the past, Wnts and their cognate receptors were also classified as either canonical or non-canonical. However, each of the 19 Wnts can bind to several Fzd receptors and each of the $10 \mathrm{Fzd}$ receptors can bind several Wnts, making Wnt signaling highly context specific (van Amerongen and Nusse 2009).

\section{II.5 Wnt signaling in BCC}

Beside Hh signaling Wnt signaling has been shown to be involved in BCC tumorigenesis (Doglioni et al. 2003; El-Bahrawy et al. 2003). In the SmoM2 BCC mouse model constitutive Hh signaling induces canonical Wnt target gene expression in tumor cells and in surrounding stromal cells. In the same 
context, inhibition of canonical Wnt signaling via Dkk1 results in suppression of tumor formation (Yang et al. 2008). Consistently, human BCC samples also exhibit nuclear accumulation of $\beta$-catenin and thus active canonical Wnt signaling (Salto-Tellez et al. 2006).

Moreover, it has been speculated that canonical Wnt signaling enhances BCC development by modulation of the Hh pathway activity (Yang et al. 2008). Indeed, Wnt signaling was shown to alter Hh signaling and vice versa. For example Gli3R is capable of inhibiting canonical Wnt signaling by antagonizing $\beta$-catenin (Ulloa et al. 2007). Interestingly, the generation of Gli3R is triggered by sequential phosphorylation involving Gsk3 which is also part of the destruction complex in canonical Wnt signaling facilitating $\beta$-catenin phosphorylation to mark it for proteasomal degradation (see section I.3) (Hui and Angers 2011). Furthermore, Sufu has been shown to bind to $\beta$-catenin leading to its nuclear export and to the inhibition of target gene expression. Mutated Sufu lacking this property leads to both active $\mathrm{Hh}$ and Wnt signaling (Taylor et al. 2004).

In development both Wnt and $\mathrm{Hh}$ signaling need to be tightly coordinated to determine the cell fate decision in various organs (Li et al. 2009). Thus, it is very likely that $\mathrm{Wnt}$ and $\mathrm{Hh}$ signaling also influence each other in BCC. Indeed, canonical Wnt signaling induces expression of GLI1 via insulin-like growth factor 2 mRNA binding protein 1 (Igf2bp1). When downregulated, Igf2bp1 suppresses the growth of BCC (Noubissi et al. 2014). Furthermore, the expression of constitutively active $\beta$-catenin in the absence of vitamin $D$ receptor leads to the formation of BCC-like lesions with up-regulated Ptch expression (Palmer et al. 2008).

In contrast, activation of Wnt/Ca2+ signaling by tumor stroma derived Wnt5a induces the differentiation and regression of BCC in a CaMKII-dependent manner (Nitzki et al. 2010).

In conclusion, these studies reflect the significant function of Wnt signaling in BCC development (Lim and Nusse 2013). 


\section{II.6 Wif1}

Wif1 was first identified as an expressed sequence tag from human retina. The initial hint that Wif1 affects Wnt signaling was derived from experiments with early Xenopus embryos when injection of RNA encoding for human WIF1 caused a partial axis duplication and abnormal somitogenesis which both were known to be controlled by the Wnt signaling pathway (Hsieh et al. 1999). Wif1 is highly conserved in human, mouse, Xenopus and zebrafish and consists of an $\mathrm{N}$-terminal signal sequence for secretion, five EGF-like repeats, a hydrophilic C-terminus and a WIF domain (WD) which is also present in the Wnt receptors Ryk/Derailed (Drl) (Patthy 2000; Yoshikawa et al. 2003) (see Fig. 10).

In the mouse the Wif1 gene is located on chromosome 10 and in humans on chromosome 12. It encodes for transcripts of 2294 or 2238 bp in length, respectively. For both mouse and human the transcript comprises 10 exons with a protein coding sequence of $1140 \mathrm{bp}$ length which is translated into a $\sim 42 \mathrm{kDa}$ protein consisting of 379 amino acids (according to NCBI CCDS database).

Human WIF1 directly binds eight Wnts (3a, 4, 5a, 7a, 9a, 11 (SurmannSchmitt et al. 2009)), to the Drosophila orthologue of Wnt, wingless, and to Xenopus Wnt8 (Hsieh et al. 1999)) by its WD and at least partially by its EGF-like domains (Malinauskas et al. 2011). Moreover, the EGF-like domains contain a HSPG-binding site suggesting that Wif1 facilitates the interaction between Wnts and HSPG in the extra cellular space. Other studies showed that the Drosophila Wif1 homolog shifted also facilitates interactions of $\mathrm{Hh}$ and HSPGs and thereby fosters Hh signaling (Glise et al. 2005; Gorfinkiel et al. 2005). More recently it was revealed that zebrafish Wif1 affects Hh signaling in Drosophila, likewise suggesting a possible role for Wif1 as a modulator of vertebrate Hh signaling (Avanesov et al. 2012).

In summary, Wif1 possibly modulates both Wnt and $\mathrm{Hh}$ signaling which are dysregulated in a variety of malignancies including BCC. 
Wif1 itself also plays a role in tumor development as it suppresses growth of many different tumors. In contrast, also an oncogenic role of Wif1 has been suggested (Huang et al. 2014). Thus, Wif1 is upregulated in some adenocarcinoma cell lines and intestinal adenomas of $A p c^{\mathrm{min} / \mathrm{t}}$ mice (Cebrat et al. 2004). However, Wif1 upregulation could be regarded as a negative feedback loop and therefore a side effect of the constitutive activity of canonical Wnt signaling without any significance for the growth of adenomas. This is due to the fact that Apc mutant cells are insensitive towards extracellular Wnt inhibitors with respect to the downregulation of $\beta$-catenin driven target gene expression.

As already mentioned, most published data show that reduced levels of Wif1 expression is associated with enhanced tumor growth, whereas Wif1 overexpression is connected with tumor growth inhibition. Thus, disruption of the murine Wif1 gene results in the development of radiation-induced osteosarcomas (Kansara et al. 2009). Promoter methylation induced downregulation of WIF1 expression is present in numerous human cancers (Mazieres et al. 2004; Haqq et al. 2005; Taniguchi et al. 2005), whereas overexpression of WIF1 inhibits the growth of lung, bladder cancer and melanoma cells (Kim et al. 2007; Lin et al. 2007; Tang et al. 2009). Recently, a rare missense mutation of Wif1 in combination with a mutation in heterogeneous nuclear ribonucleoprotein AO (HNRNPAO) has been shown to predispose members of a large family to multiple cancers (Wei et al. 2015). These data demonstrate that Wif1 plays a tumor suppressive role in a variety of tumor entities.

Previous experiments of our group showed that Wif1 is highly upregulated in BCC derived from Ptch ${ }^{\text {floxfllox}}$ CreERT2 ${ }^{\text {T/ }}$ mice (König 2012). The same applies to BCC of the SmoM2 mouse model (Youssef et al. 2012). This indicates that Wif1 is also involved in BCC development in these mouse models. 


\section{II.7 Objectives}

The aim of this thesis was to analyze the role of Wif1 in BCC. For this purpose, three experimental approaches were pursued.

First, I investigated the Wif1 expression levels in laser microdissected human BCC and compared it with human SCC and melanomas and correlated the expression levels with the degree of malignancy of the respective tumor entity.

Second, I analyzed the function of Wif1 in the BCC cell line ASZ001, which is derived from irradiated $\mathrm{Ptch}^{+/}$mice. Since ASZ001 express low levels of Wif1, I have introduced Wif1 by retroviral transduction. This allowed for analyzing the effects of Wif1 on BCC cell growth in vitro. The analyses comprised Wif1 related effects on apoptosis, cell viability, proliferation, cell cycle progression, differentiation, migratory capacity and on Wnt, $\mathrm{Hh}$ and EGFR pathway activity. Moreover, I transplanted control and Wif1 overexpressing ASZ001 (hereafter ASZ-pMSCV and ASZ-Wif1, respectively) subcutaneously in nude mice to study the effect of Wif1 on tumor growth in vivo. After excision the tumors were subjected to the same analyses as mentioned above. In addition, vascularization, EMT and stromal composition of the tumors were investigated.

Third, Ptch ${ }^{\text {flox } f l o x}$ CreERT2 ${ }^{T /}$ mice (in which BCC can be conditionally induced by injection of tamoxifen) were crossed onto a Wif1-deficient background. After BCC induction in the resulting offspring the tumors were analyzed after 45 and 90 days with respect to size and proliferative capacity.

These investigations allowed us to elucidate the effect of Wif1 on BCC development. 


\section{Material and methods}

\section{III.1 Software}

Table 1: Software

\begin{tabular}{|c|c|}
\hline Software & Supplier \\
\hline 4D v13 Volume Desktop Version 13.4 & 4D SAS, Clichy-la-Garenne, France \\
\hline ABI 3500 XL Data Collection Software v3.0 & Applied Biosystems, Darmstadt \\
\hline BD FACSDiva & Becton Dickinson GmbH, Heidelberg \\
\hline BioEdit 7.0.9 & Ibis Biosciences, Carlsbad, USA \\
\hline Endnote X5 & Thomson ISI Research Soft, California, USA \\
\hline cellSens Dimension & Olympus, Shinjuku, Japan \\
\hline CeligoS S software 2.01 & Cyntellect, San Diego, USA \\
\hline Chromas Lite 2.01 & Technelysium Pty Ltd, Helensvale, Australia \\
\hline Fiji & (Schindelin et al. 2012) \\
\hline FlowJo & Tree Star Inc., Oregon, USA \\
\hline FluorChemQ SA Version 3.2.2.0 & Cell Biosciences Inc., Heidelberg \\
\hline FreeHand MX & Adobe Systems Incorporated, San Jose, USA \\
\hline Gen5 1.11 & BioTek Instruments, Inc., Bad Friedrichshall \\
\hline GraphPad Prism 6 & GraphPad Software, La Jolla, USA \\
\hline Intas GDS & Intas Science Imaging Instruments $\mathrm{GmbH}$, Göttingen \\
\hline Microsoft Office 2007 & Microsoft Co., Redmont, USA \\
\hline MMI CellTools 4.0 & Molecular Machines \& Industries GmbH, Eching \\
\hline Photoshop 6.0 & Adobe Systems Incorporated, San Jose, USA \\
\hline SDS 2.2. & Applied Biosystems, Darmstadt \\
\hline Sequencing Analysis Software v5.4 & Applied Biosystems, Darmstadt \\
\hline
\end{tabular}

\section{III.2 Databases and web pages}

Table 2: Databases and web pages

\begin{tabular}{l|l}
\hline Database or webpage & Uniform Resource Locator \\
\hline BLAST & http://blast.ncbi.nlm.nih.gov/Blast.cgi \\
\hline Expression Atlas & http://www.ebi.ac.uk/gxa/home \\
\hline HUGO Gene Nomenclature & http://www.genenames.org/ \\
\hline MGl_3.54-Mouse Genome Informatics & http://www.informatics.jax.org/ \\
\hline
\end{tabular}




\begin{tabular}{l|l}
\hline $\begin{array}{l}\text { National Center for Biotechnology Information } \\
(\mathrm{NCBI})\end{array}$ & http://www.ncni.nlm.nih.gov/ \\
\hline Oligo Analyzer & http://eu.idtdna.com/calc/analyzer \\
\hline RT Primer DB & http://medgen.ugent.be/rtprimerdb/index.php \\
\hline The Human Protein Atlas & http://www.proteinatlas.org \\
\hline Ensembl release 64 & http://www.ensembl.org/index.html \\
\hline
\end{tabular}

\section{III.3 Equipment}

Table 3: Equipment

\begin{tabular}{|c|c|}
\hline Equipment & Supplier \\
\hline$-80^{\circ} \mathrm{C}$ Freezer (MDF-U71V) & Sanyo Electric Co. Ltd., Moriguchi, Japan \\
\hline Accu-jet & Brand GmbH \& Co. KG, Wertheim, \\
\hline Agarose gel electrophoresis chamber & Peqlab Biotechnology GmbH, Erlangen \\
\hline Arium $\AA^{\circledR} 611$ VF water purification system & Sartorius, Göttingen \\
\hline Autoclave (sanoclav) & W. Krannich GmbH \& Co. KG, Göttingen \\
\hline Biophotometer (6131) & Eppendorf AG, Hamburg \\
\hline Bunsen burner (Gasprofi 2 scs) & WLD-TEC GmbH, Göttingen \\
\hline$\left(\mathrm{CO}_{2^{-}}\right)$Incubator (6000, BBD 6220) & Kendro Laboratory Products $\mathrm{GmbH}$, Hanau \\
\hline Cold light source (KL 200) & Schott Glas, Mainz \\
\hline Cytometer Celigo S & Cyntellect, San Diego, USA \\
\hline Digital Monochrome Printer P91D & Mitsubishi, Ratingen \\
\hline Digital Photocamera (PowerShot G2) & Canon Deutschland GmbH, Krefeld \\
\hline Exposure cassettes & Sigma-Aldrich Chemistry GmbH, Steinheim \\
\hline FACS Calibur & BD Biosciences $\mathrm{GmbH}$, Heidelberg \\
\hline FluorChemQ Detection System & Cell Biosciences Inc., Heidelberg \\
\hline Freezer $\left(-20^{\circ} \mathrm{C}\right)$ & Liebherr GmbH, Ochshausen \\
\hline Fridge $\left(4^{\circ} \mathrm{C}\right)$ & Robert Bosch GmbH, Stuttgart \\
\hline Heating block (Thermomixer) & Eppendorf AG, Hamburg \\
\hline Heating stirrer (MR 3000/3001) & Heidolph Instruments, Schwabach \\
\hline High-precision scales (Sartorius Basic plus) & Sartorius AG, Göttingen \\
\hline Homogenizer (Miccra D-1) & ART Prozess- \& Labortechnik GmbH \& Co. KG, Müllheim \\
\hline Hybridization oven (HB-1000 Hybridizer) & UVP Inc., Upland, USA \\
\hline $\begin{array}{l}\text { Inverse microscope with fluorescence filter } \\
\text { (Axiovert } 25 \text {, FilterSet } 43,01,09 \text { ) }\end{array}$ & Carl Zeiss Jena GmbH, Jena \\
\hline
\end{tabular}




\begin{tabular}{|c|c|}
\hline Liquid nitrogen tank & L'air liquid S.A., Paris, France \\
\hline LSR II & BD Biosciences $\mathrm{GmbH}$, Heidelberg \\
\hline Luminometer (Synergy Mx) & BioTek Instruments, Inc., Bad Friedrichshall \\
\hline Mastercycler ep gradient S & Eppendorf AG, Hamburg \\
\hline Mercury-short-arc lamp(HBO 50W/AC) & OSRAM AG, Munich \\
\hline Microtome (HN 40) & New Brunswick Scientific GmbH, Nürtingen, \\
\hline Mini centrifuge & Carl Roth GmbH \& Co. KG, Karlsruhe \\
\hline MMI CellCut & Molecular Machines \& Industries GmbH, Eching \\
\hline Mr. Frosty ${ }^{\mathrm{TM}}$ Freezing Container & Thermo Fisher Scientific GmbH, Schwerte \\
\hline Multipette & Eppendorf AG, Hamburg \\
\hline One-channel pipettes & Eppendorf AG, Hamburg \\
\hline Paraffin dispenser (Dispenser PAG 12) & MEDITE GmbH, Burgdorf \\
\hline PCR machine (Primus HT) & MWG AG Biotech, Ebersberg \\
\hline pH-meter (inoLab pH Level 1) & WTW GmbH, Vienna, Austria \\
\hline Power supply for electrophoresis & Peqlab Biotechnology $\mathrm{GmbH}$, Erlangen \\
\hline shaking incubator & New Brunswick Scientific GmbH, Nürtingen \\
\hline shaking waterbath (1083) & GFL GmbH, Burgwedel \\
\hline Sequencer (ABI $3500 \mathrm{XL})$ & Thermo Fisher Scientific Inc., Waltham, USA \\
\hline Stereo microscope (Stemi 2000) & Carl Zeiss Jena GmbH, Jena \\
\hline Sterile bench (Euroflow class IIA) & Clean Air Techniek bv, Woerden, Netherlands \\
\hline TaqMan (ABI Prism 7900HT) & Thermo Fisher Scientific Inc., Waltham, USA \\
\hline Thermoprinter (DPU-414) & Eppendorf AG, Hamburg \\
\hline $\begin{array}{l}\text { Tissue embedding and rehydrating machine } \\
\text { (TP 1020) }\end{array}$ & Leica Microsystems $\mathrm{GmbH}$, Bensheim \\
\hline Trans-Blot SD semi-dry transfer cell & Bio-Rad Laboratories GmbH, München \\
\hline UV transilluminator & Intas Science Imaging Instruments $\mathrm{GmbH}$, Göttingen \\
\hline Vacuum pump & Schütt Labortechnik, Göttingen \\
\hline Vortexer-Genie 2 & Scientific Industries, Inc., Woburn, USA \\
\hline Weighing scale (Sartorius Basic plus) & Sartorius AG, Göttingen \\
\hline XCell4 Surelock Midi-Cell & Invitrogen $\mathrm{GmbH}$, Karlsruhe \\
\hline
\end{tabular}




\section{III.4 Consumables}

Table 4: Consumables

\begin{tabular}{|c|c|}
\hline Consumable & Supplier \\
\hline $1.5 \mathrm{ml}$ reaction tubes & Ochs $\mathrm{GmbH}$, Bovenden/Lenglern \\
\hline $1.5 \mathrm{ml}$ Safeseal Microtubes & Sarstedt AG \& Co., Nürnberg \\
\hline $13 \mathrm{ml}$ tubes & Sarstedt AG \& Co., Nürnberg \\
\hline $15 \mathrm{ml}$ tubes & Greiner Bio-One GmbH, Frickenhausen \\
\hline $2.0 \mathrm{ml}$ reaction tubes & Sarstedt AG \& Co., Nürnberg \\
\hline 24-well cell culture plate & Corning Inc., Corning, USA \\
\hline 384-well Optical Reaction Plate & Thermo Fisher Scientific Inc., Waltham, U.S.A. \\
\hline $50 \mathrm{ml}$ tubes & Greiner Bio-One GmbH, Frickenhausen \\
\hline 6-well cell culture plate & Corning Inc., Corning, USA \\
\hline 96-well Assay Plate & Corning Inc., Corning, USA \\
\hline 96-well Optical Reaction Plate & Thermo Fisher Scientific Inc., Waltham, USA. \\
\hline Balance oeco multi-function paper & inapa tecno, Hamburg \\
\hline BD Discardit ${ }^{I M} \|(2,10,20 \mathrm{ml})$ & BD Biosciences $\mathrm{GmbH}$, Heidelberg \\
\hline BD Microfine + Demi & BD Biosciences $\mathrm{GmbH}$, Heidelberg \\
\hline BD Plastipak & BD Biosciences $\mathrm{GmbH}$, Heidelberg \\
\hline BD Plastipak $1 \mathrm{ml}$ Sub-Q & BD Biosciences $\mathrm{GmbH}$, Heidelberg \\
\hline Blotting paper (GB 33 B003) & Heinemann Labortechnik GmbH, Duderstadt \\
\hline $\begin{array}{l}\text { Cell culture dishes, } 35 \mathrm{~mm}, 100 \mathrm{~mm} \text {, (Nunclon } \\
\text { Surface) }\end{array}$ & Nunc GmbH \& Co.KG, Wiesbaden \\
\hline Cell culture inserts, 24 well, $8.0 \mu \mathrm{m}$ & Corning Inc., Corning, USA \\
\hline Cell culture inserts, 24 well, $0.4 \mu \mathrm{m}$ & Corning Inc., Corning, USA \\
\hline Cell scraper & Sarstedt AG \& Co., Nürnberg \\
\hline Combitips plus $(0.2,0.5,2.5,5,10 \mathrm{ml})$ & Eppendorf AG, Hamburg \\
\hline Coverslips & Menzel GmbH \& Co.KG, Braunschweig \\
\hline Cryo Pure & Sarstedt AG \& Co., Nürnberg \\
\hline Cuvettes (UVette) & Carl Roth GmbH \& Co. KG, Karlsruhe \\
\hline Filter tips $(10 \mu \mathrm{l})$ & Sarstedt AG \& Co., Nürnberg \\
\hline Filter tips $(100 \mu \mathrm{l}, 200 \mu \mathrm{l}, 1000 \mu \mathrm{l})$ & Kisker Biotech GmbH \& Co. KG, Steinfurt \\
\hline Flow cytometry tubes & Sarstedt AG \& Co., Nürnberg \\
\hline Fluted filters & Sartorius AG, Göttingen \\
\hline Glassware & Schott AG, Mainz \\
\hline Hyperfilm ECL & Amersham Biosciences Europe $\mathrm{GmbH}$, Freiburg \\
\hline
\end{tabular}




\begin{tabular}{|c|c|}
\hline Miscroscope slides (SuperFrost Plus) & Menzel GmbH \& Co.KG, Braunschweig \\
\hline MMI MembraneSlides & Molecular Machines \& Industries $\mathrm{GmbH}$, Eching \\
\hline MultiScreen $_{\mathrm{HTS}}-\mathrm{HV}$ plate & Millipore GmbH, Schwalbach am Taunus \\
\hline Neubauer counting chamber & Brand GmbH \& Co KG, Wertheim \\
\hline Nitrocellulose membrane(Hybond ECL) & GE Healthcare Europe GmbH, Freiburg \\
\hline NuPAGE Novex 4-12\% Bis-Tris Midi Gel & Invitrogen $\mathrm{GmbH}$, Karlsruhe \\
\hline Pasteur pipettes & Brand GmbH \& Co.KG, Wertheim \\
\hline $\begin{array}{l}\text { PCR-Reaction tubes (ThermoFast } 96, \\
\text { nonskirted, natural domed cap strips, natural) }\end{array}$ & Sarstedt AG \& Co., Nürnberg \\
\hline Petri dishes & Ochs GmbH, Bovenden/Lenglern \\
\hline Pipette tips $(10 \mu \mathrm{l}, 200 \mu \mathrm{l})$ & Ochs $\mathrm{GmbH}$, Bovenden/Lenglern \\
\hline Pipette tips (1000 $\mu$ l) & Sarstedt AG \& Co., Nürnberg \\
\hline Pipette tips $(20 \mu l)$ & Sarstedt AG \& Co., Nürnberg \\
\hline Protein concentrators, $20 \mathrm{ml}$, 9k MWKO & Thermo Fisher Scientific Inc., Waltham, USA \\
\hline QPCR Adhesive Clear Seal & 4titude Ltd., Berlin \\
\hline Scalpel blade \#10, \#24 & Aesculap AG \& Co.KG, Tuttlingen \\
\hline Serological pipettes (5 ml, 10 ml, 25 ml) & Sarstedt AG \& Co., Nürnberg \\
\hline Sterile filter & Omnilab-Krannich, Göttingen \\
\hline Syringe $30,50 \mathrm{ml}$ & Terumo Medical Corp., Elkton, USA \\
\hline Tissue Culture Plate 6-Well & Sarstedt AG \& Co., Nürnberg \\
\hline Tissue Culture Plate 24-Well & Sarstedt AG \& Co., Nürnberg \\
\hline
\end{tabular}

\section{III.5 Chemicals and reagents}

\section{All standard chemicals not listed here were obtained from AppliChem $\mathrm{GmbH}$, Darmstadt, Carl Roth GmbH \& Co. KG, Karlsruhe, or from Sigma-Aldrich Chemistry $\mathrm{GmbH}$, Steinheim.}

Table 5: Chemicals and reagents

\begin{tabular}{l|l}
\hline Chemicals and reagnets & Supplier \\
\hline 100 bp plus and 1 kb DNA Ladder & Fermentas GmbH, St. Leon-Rot \\
\hline Agarose & Bio-Budget Technologies GmbH, Krefeld \\
\hline BM Purple & Roche Diagnostics GmbH, Mannheim \\
\hline Boric acid & MP Biomedicals LLC, Illkirch, France \\
\hline cOmplete, protease inhibitor cocktail & Roche Diagnostics GmbH, Mannheim \\
\hline
\end{tabular}




\begin{tabular}{|c|c|}
\hline Deoxyribonucleotide triphosphate (dNTP) & Roche Diagnostics $\mathrm{GmbH}$, Mannheim \\
\hline Dithiotreitol, 100mM (DTT) & Invitrogen $\mathrm{GmbH}$, Karlsruhe \\
\hline DNase/Rnase-free water & GIBCO Invitrogen $\mathrm{GmbH}$, Karlsruhe \\
\hline Ethidium bromide $(0.07 \%)$ & inna-TRAIN-Diagnostics, Kronberg \\
\hline EtOH $99 \%$ & J.T. Baker B.V., Deventer, Netherlands \\
\hline EtOH $99 \%$ denatured & CVH Chemie-Vertrieb GmbH \& Co. Hannover KG, Hannover \\
\hline Formamide & Acros Organics b.v.b.a, Geel, Belgium \\
\hline Glycergel mounting medium & Dako GmbH, Hamburg \\
\hline Hematoxilin solution, MAYER & Medite $\mathrm{GmbH}$, Burgdorf \\
\hline HEPES buffer solution (1M) & GIBCO Invitrogen GmbH, Karlsruhe \\
\hline I-Block & Tropix, Bedford, USA \\
\hline Indo-1 AM & Thermo Fisher Scientific Inc., Waltham, USA \\
\hline ionomycin & Sigma-Aldrich Chemistry GmbH, Steinheim \\
\hline Matrigel, phenol red free & BD Biosciences $\mathrm{GmbH}$, Heidelberg \\
\hline NuPAGE MES SDS Running Buffer, $20 \mathrm{x}$ & Invitrogen $\mathrm{GmbH}$, Karlsruhe \\
\hline PBS-Tablets & GIBCO Invitrogen $\mathrm{GmbH}$, Karlsruhe \\
\hline Pertex mounting medium & Medite Medizintechnik GmbH, Burgdorf \\
\hline PhosStop & Roche Diagnostics GmbH, Mannheim \\
\hline Pluronic F127 & Thermo Fisher Scientific Inc., Waltham, USA \\
\hline SeeBlue ${ }^{\circledR}$ Plus2 Pre-Stained Standard & Invitrogen $\mathrm{GmbH}$, Karlsruhe \\
\hline illustra Sephadex G-50 DNA Grade F & GE Healthcare Europe GmbH, Freiburg \\
\hline TRIzol Reagent & Invitrogen $\mathrm{GmbH}$, Karlsruhe \\
\hline Xylene & J.T. Baker B.V., Deventer, Netherlands \\
\hline
\end{tabular}

\section{III.6 Ready-to-use reaction systems}

Table 6: Ready-to-use reaction systems

\begin{tabular}{l|l}
\hline Reaction system & Supplier \\
\hline $\begin{array}{l}\text { Amersham ECL Plus }{ }^{\mathrm{TM}} \text { Western Blotting } \\
\text { Detection Reagents }\end{array}$ & GE Healthcare Europe GmbH, Freiburg \\
\hline $\begin{array}{l}\text { Arcurus Paradise PLUS FFPE RNA Isolation Kit } \\
\text { (ABI; KIT0312I) }\end{array}$ & Thermo Fisher Scientific Inc., Waltham, USA \\
\hline BigDye Terminator v3.1 Cycle Sequencing Kit & Thermo Fisher Scientific Inc., Waltham, USA \\
\hline Cell Proliferation ELISA, BrdU & Roche Diagnostics GmbH, Mannheim \\
\hline Cell Proliferation Reagent WST-1 & Roche Diagnostics GmbH, Mannheim \\
\hline
\end{tabular}




\begin{tabular}{|c|c|}
\hline DeadEnd ${ }^{\mathrm{TM}}$ Colorimetric TUNEL System & Promega GmbH, Mannheim \\
\hline Dual-Luciferase Reporter Assay System & Promega GmbH, Mannheim \\
\hline FITC Annexin V & BD Biosciences GmbH, Heidelberg \\
\hline HiPure Plasmid DNA Purification Kit & Invitrogen $\mathrm{GmbH}$, Karlsruhe \\
\hline High Pure PCR Cleanup Micro Kit & Roche Diagnostics $\mathrm{GmbH}$, Mannheim \\
\hline MicroSpin G50 Columns & Amersham Biosciences Europe $\mathrm{GmbH}$, Freiburg \\
\hline Pierce BCA Protein Assay Kit & Fisher Scientific GmbH， Schwerte \\
\hline $\begin{array}{l}\text { Platiunum SYBR Green qPCR SuperMix-UDG } \\
\text { with ROX }\end{array}$ & Invitrogen $\mathrm{GmbH}$, Karlsruhe \\
\hline QuantiTect PCR Probe Kit & Qiagen GmbH, Hilden \\
\hline QuantiTect SYBR Green PCR & Qiagen GmbH, Hilden \\
\hline Roti-Fect transfection reagent & Carl Roth GmbH \& Co. KG, Karlsruhe \\
\hline RNeasy Fibrous Tissue Kit & Qiagen GmbH, Hilden \\
\hline SuperScriptll Reverse Transcriptase & Invitrogen $\mathrm{GmbH}$, Karlsruhe \\
\hline TransITß-LT1 Transfection Reagent & Mirus Bio LLC., Madison, USA \\
\hline
\end{tabular}

\section{III.7 Buffers and solutions}

Buffers and solutions were prepared using double-distilled water.

AEC chromogen, $\mathrm{pH} 5.2$

Blotting buffer

Boric acid, $\mathrm{pH} 5.1$
$30 \mathrm{mM}$ acetic acid

$70 \mathrm{mM}$ sodium acetate trihydrate

16 mM 3-amino-9 ethylcarbazole

(dissolved in dimethyl formamide)

$6 \%(w / v)$ Tris base

$3 \%(w / v)$ glycine

$0.075 \%$ SDS

$20 \%(v / v)$ methanol

0.2 M Boric acid 
BSA/sodium azide solution

Citrate buffer, pH 3.0

Citrate buffer, $\mathrm{pH} 6.0$

Cresol solution

Deoxyribonukleotidtriphosphate (dNTP)-mix

DNase I buffer

Eosin

Krebs Ringer solution

\author{
$0.1 \%(v / v)$ Tween-20 \\ $0.02 \%(w / v)$ sodium azide \\ $3 \%(w / v) B S A$ \\ in $1 \times$ TBS
}

$10 \mathrm{mM}$ sodium citrate

$10 \mathrm{mM}$ sodium citrate

$0.1 \%(w / v)$ Cresol

in saturated sucrose-solution

$10 \mathrm{mM}$ dATP

$10 \mathrm{mM}$ dTTP

$10 \mathrm{mM}$ dGTP

$10 \mathrm{mM}$ dCTP

40 mM Tris- $\mathrm{HCl}(\mathrm{pH} 7.9)$

$10 \mathrm{mM} \mathrm{NaCl}$

$6 \mathrm{mM} \mathrm{MgCl}_{2}$

$10 \mathrm{mM} \mathrm{CaCl}_{2}$

$1 \%(w / v)$ eosin y (water soluble)

$80 \%(v / v)$ ethanol

10 mM HEPES (pH 7.0)

$140 \mathrm{mM} \mathrm{NaCl}$

$4 \mathrm{mM} \mathrm{KCl}$

$1 \mathrm{mM} \mathrm{MgCl}_{2}$

$1 \mathrm{mM} \mathrm{CaCl}_{2}$

$10 \mathrm{mM}$ glucose 
Krebs Ringer solution $\left(\mathrm{Ca}^{2+}\right.$-free $)$

Modified RIPA buffer

Paraformaldehyde solution (PFA)

Phosphate-buffered

sodium chloride-solution

(PBS), $10 x, \mathrm{pH} 7.4$

PBST (washing buffer)

Proteinase $\mathrm{K}$ buffer

SDS loading buffer, $6 \mathrm{x}$

in upper gel buffer
10 mM HEPES (pH 7.0)

$140 \mathrm{mM} \mathrm{NaCl}$

$4 \mathrm{mM} \mathrm{KCl}$

$1 \mathrm{mM} \mathrm{MgCl}_{2}$

$0.5 \mathrm{mM}$ EGTA

$10 \mathrm{mM}$ glucose

$50 \mathrm{mM}$ Tris/HCl pH 7.4

$1 \%$ NP-40

$0.25 \%$ Na-Deoxycholat

$150 \mathrm{mM} \mathrm{NaCl}$

1 mM EDTA

1 protease inhibitor cocktail tablet per $10 \mathrm{ml}$

1 PhosSTOP tablet per $10 \mathrm{ml}$

$4 \%$ (w/v) Paraformaldehyde

in $1 \times$ PBS

$1.4 \mathrm{M} \mathrm{NaCl}$

$27 \mathrm{mM} \mathrm{KCl}$

$15 \mathrm{mM} \mathrm{KH}_{2} \mathrm{PO}_{4}$

$65 \mathrm{mM} \mathrm{Na}_{2} \mathrm{HPO}_{4}$

$0.1 \%(v / v)$ Tween-20

in $1 \times$ PBS

$50 \mathrm{mM}$ Tris/HCl pH 8.0

5 mM EDTA

$35 \%$ (v/v) glycerol

$9 \%(w / v)$ SDS

$8.5 \%(w / v)$ DTT

$0.1 \%(w / v)$ bromophenol blue 
STE-buffer

Tris-EDTA buffer pH 9,0 (TE)

Tris-boric acid-EDTA-solution, $10 \times(\mathrm{TBE})$
$50 \mathrm{mM}$ Tris/HCl pH 8.0

$100 \mathrm{mM} \mathrm{NaCl}$

1 mM EDTA

$1 \%(w / v)$ SDS

$10 \mathrm{mM}$ Tris

1 mM EDTA

890 mM Tris/HCl pH 8.0

$730 \mathrm{mM}$ boric acid

$12.5 \mathrm{mM}$ EDTA

Tris-buffered sodium chloride-solution, $0.5 \mathrm{M}$ Tris/ $\mathrm{HCl} \mathrm{pH} 7.4$

$10 \times($ TBS $)$

$1.5 \mathrm{M} \mathrm{NaCl}$

TBST

$0.1 \%(v / v)$ Tween-20

in $1 \times$ TBS

Upper gel buffer

$6 \%(\mathrm{w} / \mathrm{v})$ Tris base $\mathrm{pH} 6.8$

$4 \%(v / v)$ SDS

\section{III.8 Media}

\section{III.8.1 Media for bacteria culture}

For cultivation of bacteria (E. coli $\mathrm{DH} 5 \alpha$, Invitrogen $\mathrm{GmbH}$, Karlsruhe) lysogeny broth-medium ((LB-medium;1 \% (w/v) tryptone, $0.5 \%$ (w/v) yeast extract, $1 \%(\mathrm{w} / \mathrm{v}), \mathrm{NaCl} ; \mathrm{pH} 7.0))$ was used. LB-medium was prepared with double-distilled water, autoclaved and stored at $4^{\circ} \mathrm{C}$. Selection for adequate resistance genes was achieved by addition of $100 \mu \mathrm{g} / \mathrm{ml}$ ampicillin (stock concentration: 100 mg/ml; Carl Roth $\mathrm{GmbH} \&$ Co. KG, Karlsruhe) or 50 mg/ml kanamycin (stock concentration: $10 \mathrm{mg} / \mathrm{ml}$, Sigma-Aldrich Chemistry $\mathrm{GmbH}$, Steinheim) 


\section{III.8.2 Agar plates}

For preparation of LB-agar plates $1.5 \%(w / v)$ agar was added to LB-medium and autoclaved. After cooling down to $55^{\circ} \mathrm{C}$ the adequate antibiotic (100 $\mu \mathrm{g} / \mathrm{ml}$ ampicillin or $50 \mu \mathrm{g} / \mathrm{ml}$ kanamycin) was added and LB-agar was plated in $10 \mathrm{~cm}$ Petri dishes. Agar plates were stored at $4^{\circ} \mathrm{C}$ in sterile plastic bags.

\section{III.8.3}

\section{Media and reagents for cultivation of eukaryotic cell lines}

All Media and supplements including antibiotics used for selection of resistant clones that were used for cultivation of eukaryotic cell lines are listed in Table 7. For the preparation of $\mathrm{Ca}^{2+}$-chelexed and heat-inactivated FCS, $50 \mathrm{ml}$ FCS were heat-inactivated by incubation for $30 \mathrm{~min}$ at $56^{\circ} \mathrm{C}$. Afterwards, $10 \mathrm{~g}$ Chelex 100 were added and stirred for $60 \mathrm{~min}$ at room temperature (RT) on a magnetic stirrer to eliminate $\mathrm{Ca}^{2+}$-ions. Subsequently, the FCS was sterile filtered with an UV-sterilized fluted filter and additionally with a $0.2 \mu \mathrm{m}$ sterile filter and stored at $-20^{\circ} \mathrm{C}$.

Table 7: Cell culture media and supplements for eukaryotic cell lines

\begin{tabular}{l|l}
\hline Medium or reagent & Supplier \\
\hline 154CF Medium & Gibco, Invitrogen GmbH, Karlsruhe \\
\hline Accutase & PAA Laboratories GmbH, Pasching \\
\hline Chelex 100 Resin Chelating lon Exchanger Resin & BioRad Laboratories Inc., Hercules, USA \\
\hline Dulbecco's Modified Eagle Medium (DMEM) & Gibco, Invitrogen $\mathrm{GmbH}$, Karlsruhe \\
\hline Fetal calf serum (FCS) & Gibco, Invitrogen GmbH, Karlsruhe \\
\hline G 418 disulfate salt solution $(50 \mathrm{mg} / \mathrm{ml})$ & Sigma-Aldrich Chemistry GmbH, Steinheim \\
\hline HEPES (1M) & Gibco, Invitrogen GmbH, Karlsruhe \\
\hline Horse serum & Gibco, Invitrogen GmbH, Karlsruhe \\
\hline RPMl $1640 \mathrm{Medium}$ & Gibco, Invitrogen GmbH, Karlsruhe \\
\hline Penicillin (10.000 U/ml)/Streptomycin (10 mg/ml) (P/S) & PAN Biotech GmbH, Aidenbach \\
\hline Puromycin dihydrochloride (10 mg/ml) & Sigma-Aldrich Chemistry GmbH, Steinheim \\
\hline S.O.C. medium & Invitrogen GmbH, Karlsruhe \\
\hline
\end{tabular}




\section{III.9 Biological material}

\section{III.9.1 Bacterial strain}

For transformation and amplification of plasmid DNA the E. coli strain DH5a was used.

\section{III.9.2 Eukaryotic cell lines}

Eukaryotic cell lines B9, C5N, NIH/3T3, NIH/3T3-Wnt5a, HEK-293 and Wnt-3A $L$ and $L$ cells were cultured in Dulbecco's Modified Eagle Medium (DMEM) supplemented with $10 \%$ FCS and $1 \%$ penicillin/streptomycin (P/S). The cell line ASZ001 was cultured in medium 154CF containing $0.05 \mathrm{M}$ $\mathrm{CaCl}_{2}, 2 \%$ heat-inactivated and $\mathrm{Ca}^{2+}$-chelexed FCS and $1 \% \mathrm{P} / \mathrm{S}$. The respective stably transfected cell lines were cultured in the presence of an adequate antibiotic (see Table 8).

Table 8: Eukaryotic cell lines

1: Designation of cell line; 2: Origin of cell line; 3: Culture medium; 4: Source of cell line/reference; Abbreviations: BCC: basal cell carcinoma; FCS: fetal calf serum; P/S: penicillin/streptomycin; DMEM: Dulbecco`s Modified Eagle Medium; Wnt3a: wingless-related MMTV integration site 3A;

\begin{tabular}{l|l|l|l}
\hline 1 & 2 & 3 & 4 \\
\hline ASZ001 & murine BCC & $\begin{array}{l}\text { 154CF, 2\% chelexed } \\
\text { FCS, 1\% P/S }\end{array}$ & (So et al. 2006) \\
\hline B9 & $\begin{array}{l}\text { murine adult } \\
\text { fibroblasts }\end{array}$ & $\begin{array}{l}\text { DMEM, 10\% FCS, 1\% } \\
\text { P/S }\end{array}$ & F. Nitzki, dissertation, 2008 \\
\hline C5N & murine keratinocytes & $\begin{array}{l}\text { DMEM (lacking } \\
\text { pyruvate), 10\% FCS, 1 }\end{array}$ & (Kulesz-Martin et al. 1983) \\
\hline HEK-293 & $\begin{array}{l}\text { human embryonic } \\
\text { kidney }\end{array}$ & $\begin{array}{l}\text { DMEM, 10\% FCS, 1\% } \\
\text { P/S }\end{array}$ & $\begin{array}{l}\text { ATCC LGC Promochem, Wesel; CRL- } \\
1573\end{array}$ \\
\hline L929 & murine fibroblast & $\begin{array}{l}\text { RPMl 1640 10\% FCS, 1 } \\
\% \text { P/S }\end{array}$ & $\begin{array}{l}85011425, \text { Sigma Aldrich Chemistry } \\
\text { GmbH, Steinheim }\end{array}$ \\
\hline
\end{tabular}




\begin{tabular}{|c|c|c|c|}
\hline $\mathrm{L}$ cells & murine fibroblasts & $\begin{array}{l}\text { DMEM, } 10 \% \text { FCS, } 1 \% \\
\text { P/S }\end{array}$ & ATCC $^{\circledast}$ CRL-2648 \\
\hline $\begin{array}{l}\text { Wnt-3A L } \\
\text { cells }\end{array}$ & $\begin{array}{l}\text { murine fibroblasts } \\
\text { stably overexpressing } \\
\text { Wnt3a }\end{array}$ & $\begin{array}{l}\text { DMEM, } 10 \% \text { FCS, } 1 \% \\
\text { P/S, } 0.4 \mu \mathrm{g} / \mathrm{ml} \mathrm{G} 418\end{array}$ & ATCC $^{\circledR}$ CRL-2647'M \\
\hline $\mathrm{NIH} / 3 \mathrm{~T} 3$ & $\begin{array}{l}\text { murine embryonic } \\
\text { fibroblasts }\end{array}$ & $\begin{array}{l}\text { DMEM, } 10 \% \text { FCS, } 1 \% \\
\text { P/S }\end{array}$ & $\begin{array}{l}\text { ATCC LGC Promochem, Wesel; CRL- } \\
1658\end{array}$ \\
\hline $\begin{array}{l}\text { NIH/3T3- } \\
\text { Wnt5a }\end{array}$ & $\begin{array}{l}\text { see above, stably } \\
\text { overexpressing Wnt5a }\end{array}$ & $\begin{array}{l}\text { DMEM, } 10 \% \text { FCS, } 1 \% \\
\text { P/S, } 0.4 \mu \mathrm{g} / \mathrm{ml} \mathrm{G} 418\end{array}$ & (Kispert et al. 1998) \\
\hline Platenum-E & $\begin{array}{l}\text { retroviral packaging } \\
\text { cell line }\end{array}$ & $\begin{array}{l}\text { DMEM, } 10 \% \text { FCS), } \\
1 \mu \mathrm{g} / \mathrm{mL} \text { puromycin, } \\
10 \mu \mathrm{g} / \mathrm{mL} \text { blasticidin, } \\
1 \% \mathrm{P} / \mathrm{S}\end{array}$ & RV-101, cell biolabs, Inc. \\
\hline
\end{tabular}

\section{III.9.3 Mouse lines}

Table 9: Mouse lines

1: Designation of mouse line; 2: strain background; 3: Genetic modification and use; 4: Source

\begin{tabular}{|c|c|c|c|}
\hline 1 & 2 & 3 & 4 \\
\hline nude mice & outbred & athymic; allograft & animal keeping \\
\hline $\operatorname{Ptch}^{f l o x}$ & $\begin{array}{l}\text { C57BL/6N, } \\
129 / \mathrm{Sv}\end{array}$ & $\begin{array}{l}\text { Floxed exons } 8 \text { and } 9 \text { of the murine } \\
\text { Ptch gene; for conditional } \\
\text { Ptch gene deletion }\end{array}$ & (Uhmann et al. 2007) \\
\hline $\begin{array}{l}\text { ROSA26- } \\
\text { CreERT2 }\end{array}$ & $129 S v$ & $\begin{array}{l}\text { ubiquituous tamoxifen-inducible CreERT2- } \\
\text { recombinase expression under control of } \\
\text { ROSA26 promotor }\end{array}$ & $\begin{array}{l}\text { generous gift from A. Berns, } \\
\text { Netherlands }\end{array}$ \\
\hline Wif1 $1^{--}$ & $\begin{array}{l}\text { C57BL/6, } \\
\text { 129/Sv }\end{array}$ & $\begin{array}{l}\text { Tau-LacZ reporter cassette integrated in Exon } 1 \\
\text { causes a frameshift and thereby abrogates Wif1 } \\
\text { expression }\end{array}$ & (Kansara et al. 2009) \\
\hline
\end{tabular}

All experiments using animals were performed in compliance with all legal and ethical requirements.

\section{III.10 Plasmids}

Plasmids listed in Table 9 were either used for transfection, nucleofection or transduction of eukaryotic cell lines. 
Table 10: Plasmids

1: Designation of vector; 2: Purpose; 3: Designation of vector backbone if different from 1; 4: Supplier of vector backbone or reference (if applicable);

\begin{tabular}{|c|c|c|c|}
\hline 1 & 2 & 3 & 4 \\
\hline pCR3.1 & Dual-Luciferase assay & & Invitrogen $\mathrm{GmbH}$, Karlsruhe \\
\hline pCl-neo- $\beta$-cateninS33Y & Dual-Luciferase assay & pCl-neo & (Morin et al. 1997) \\
\hline pcDNA3-Wif1 & Wif1 overexpression & pcDNA3 & (Becker 2011) \\
\hline pGFPmax & Nucleofection control & & Lonza Group AG, Basel, Switzerland \\
\hline pMSCVpuro & $\begin{array}{l}\text { Wif1 overexpression } \\
\text { control plasmid }\end{array}$ & & Clontech Laboratories, Inc. \\
\hline pMSCVpuro-Wif1 & Wif1 overexpression & pMSCVpuro & this thesis \\
\hline$p R L-C M V$ & Dual-Luciferase assay & & Promega GmbH, Mannheim \\
\hline SuperTOPFlash & Dual-Luciferase assay & pTA-Luc & (Korinek et al. 1997) \\
\hline SuperFOPFlash & Dual-Luciferase assay & pTA-Luc & (Korinek et al. 1997) \\
\hline pEGFP-N1 & $\begin{array}{l}\text { Transfection efficiency } \\
\text { control }\end{array}$ & & $\begin{array}{l}\text { Takara Bio Europe/Clontech, Saint- } \\
\text { Germain-en-Laye, France }\end{array}$ \\
\hline
\end{tabular}

\section{III.11 Synthetic oligonucleotides}

Synthetic DNA-oligonucleotides were ordered from Eurofins MWG Operon, Ebersberg. The stock concentration of DNA-oligonucleotides was set to $100 \mu \mathrm{M}$ using RNase- and DNase-free water. For all PCR reactions a $10 \mu \mathrm{M}$ solution was used.

\section{III.11.1 Synthetic DNA-oligonucleotides for qRT-PCR}

Since embryos express of a broad range of genes, cDNAs made from mouse embryos of different ages (see Table 11) were used a) to establish the qRT-PCR assay for the expression analysis of a specific gene and b) to set up the standard curve to calculate the expression level of the respective gene. The age of an embryo used for the synthesis of cDNA was chosen according to the expression profile of respective mRNA reported in the database MGI and Expression Atlas. 
Table 11: Oligonucleotides for qRT-PCR analyses

1: Designation of analysed transcript; 2 : Designation of oligonucleotides; 3: Oligonucleotide sequence $\left(5^{\prime} \rightarrow 3^{\prime}\right)$; 4 : control cDNAs, 10.5, 12.5, 13.5: Age of mouse embryos in days post coitum (dpc) for generation of control cDNA, BCC: Basal Cell Carcinoma, HEK LiCl: HEK-293 treated for $12 \mathrm{~h}$ with $50 \mathrm{nM} \mathrm{LiCl}$; 5: References; 6: Supplier of utilized SYBR Green (determining program used, see. III.14.2.3).

\begin{tabular}{|c|c|c|c|c|c|}
\hline 1 & 2 & 3 & 4 & 5 & 6 \\
\hline $18 S$ & $\begin{array}{l}18 S \text { forw } \\
18 S \text { rev2 }\end{array}$ & $\begin{array}{l}\text { CGCAAATTACCCACTCCCG } \\
\text { TTCCAATTACAGGGCCTCGAA }\end{array}$ & 13.5 & $\begin{array}{l}\text { (Nitzki et al. } \\
\text { 2010) }\end{array}$ & $\begin{array}{l}\text { Invitrogen, } \\
\text { Qiagen }\end{array}$ \\
\hline Axin2 & $\begin{array}{l}m A x i n 2-F \\
m A x i n 2-R\end{array}$ & $\begin{array}{l}\text { TGTGAGATCCACGGAAACAGCT } \\
\text { TGTCCGTCATGGACATGGAATC }\end{array}$ & 12.5 & $\begin{array}{l}\text { (Nitzki et al. } \\
\text { 2010) }\end{array}$ & Qiagen \\
\hline AXIN2 & $\begin{array}{l}h A X I N 2-F \\
h A X I N 2-R\end{array}$ & $\begin{array}{l}\text { GCCAACGACAGTGAGATATCC } \\
\text { CTCGAGATCAGCTCAGCTGCA }\end{array}$ & $\begin{array}{l}\text { HEK } \\
\mathrm{LiCl}\end{array}$ & this thesis & Invitrogen \\
\hline Cdh1 & $\begin{array}{l}m C d h 1-F 3 \\
m C d h 1-R 3\end{array}$ & $\begin{array}{l}\text { AGGAGCTGGAGCCTGAGTC } \\
\text { CGAAAAGAAGGCTGTCCTTG }\end{array}$ & 13.5 & this thesis & Invitrogen \\
\hline Cdh2 & $\begin{array}{l}\text { Cdh2- } F \\
\text { Cdh2- } R\end{array}$ & $\begin{array}{l}\text { TTACAGCGCAGTCTTACCGA } \\
\text { CGTCCACCTTGAAATCTGCT }\end{array}$ & 10.5 & this thesis & Invitrogen \\
\hline$c-M y c$ & $\begin{array}{l}m c-M y c F \\
m c-M y c R\end{array}$ & $\begin{array}{l}\text { TAGTGCTGCATGAGGAGAC } \\
\text { CTCCACAGACACCACATCA }\end{array}$ & 12.5 & $\begin{array}{l}\text { working group } \\
\text { Hahn, F. Nitzki }\end{array}$ & Qiagen \\
\hline Fn1 & $\begin{array}{l}F n 1-F \\
F n 1-R\end{array}$ & $\begin{array}{l}\text { GGTCAGTCCTACAAGATTGGC } \\
\text { TCCAATCCTATAGGATGTCCG }\end{array}$ & 10.5 & this thesis & Invitrogen \\
\hline Gli1 & $\begin{array}{l}\text { mGli1-tq-f } \\
m \text { Gli1-tq-r }\end{array}$ & $\begin{array}{l}\text { TACATGCTGGTGGTGCACATG } \\
\text { ACCGAAGGTGCGTCTTGAGG }\end{array}$ & 12.5 & $\begin{array}{l}\text { working group } \\
\text { Hahn, F. Nitzki }\end{array}$ & Qiagen \\
\hline Hprt & $\begin{array}{l}m H p r t-F w-Q \\
m H p r t-R e v-Q\end{array}$ & $\begin{array}{l}\text { AGCCCCAAAATGGTTAAGGTTGC } \\
\text { TTGCAGATTCAACTTGCGCTCAT }\end{array}$ & 13.5 & $\begin{array}{l}\text { working group } \\
\text { Hahn, B. Linder }\end{array}$ & $\begin{array}{l}\text { Invitrogen, } \\
\text { Qiagen }\end{array}$ \\
\hline$I V I$ & $\begin{array}{l}|V|-1-F 1 \\
|V|-1-R 1\end{array}$ & $\begin{array}{l}\text { ССTCCTGTGAGTTTGTTTGGTCT } \\
\text { CTGAGGATATGATCTGGAGAAC }\end{array}$ & BCC & $\begin{array}{l}\text { working group } \\
\text { Hahn, B. Linder }\end{array}$ & Invitrogen \\
\hline K1 & $\begin{array}{l}K 1-F \\
K 1-R\end{array}$ & $\begin{array}{l}\text { TCAACGTTGAGGTTGACCCTC } \\
\text { ACCTTCCTTCTGAGGATGCTG }\end{array}$ & $\mathrm{BCC}$ & $\begin{array}{l}\text { (Nitzki et al. } \\
\text { 2010) }\end{array}$ & Qiagen \\
\hline K10 & $\begin{array}{l}K 10-F \\
K 10-R\end{array}$ & $\begin{array}{l}\text { GGATGCTGAAGAGTGGTTCAA } \\
\text { TCTGTTTCTGCCAAGGAGGCT }\end{array}$ & $\mathrm{BCC}$ & $\begin{array}{l}\text { (Nitzki et al. } \\
\text { 2010) }\end{array}$ & Qiagen \\
\hline Lor & $\begin{array}{l}\text { Lor-F1 } \\
\text { Lor-R1 }\end{array}$ & $\begin{array}{l}\text { САСTCATCTTCССTGGTGCTTC } \\
\text { GTCTTTCCACAACCCACAGGAG }\end{array}$ & $\mathrm{BCC}$ & $\begin{array}{l}\text { working group } \\
\text { Hahn, B. Linder }\end{array}$ & Invitrogen \\
\hline Tbp & $\begin{array}{l}\text { mTBP-Q-Fwd } \\
\text { mTBP-Q-Rev }\end{array}$ & $\begin{array}{l}\text { CACCAATGACTCCTATGACCCCTA } \\
\text { CAGTTGTCCGTGGCTCTCTTATTC }\end{array}$ & 13.5 & $\begin{array}{l}\text { working group } \\
\text { Hahn, B. Linder }\end{array}$ & $\begin{array}{l}\text { Invitrogen, } \\
\text { Qiagen }\end{array}$ \\
\hline Tgm & $\begin{array}{l}\text { Tgm1-F } \\
\text { Tgm1-R }\end{array}$ & $\begin{array}{l}\text { GCAGTGGTGTAAATGCAGCTGG } \\
\text { ATGAGGAGCTCAAGGGCAATGC }\end{array}$ & $\mathrm{BCC}$ & $\begin{array}{l}\text { working group } \\
\text { Hahn, S. König }\end{array}$ & Qiagen \\
\hline Wif1 & $\begin{array}{l}m W i f 1-F \\
m W i f 1-R\end{array}$ & $\begin{array}{l}\text { TCCTGTCAATATCCACTCCATGAA } \\
\text { CTGATGCCTTGTGAGGCACT }\end{array}$ & 12.5 & $\begin{array}{l}\text { working group } \\
\text { Hahn, S. König }\end{array}$ & Invitrogen \\
\hline WIF1 & $\begin{array}{l}h W I F 1-F 2 \\
h W I F 1-R 2\end{array}$ & $\begin{array}{l}\text { ACAACCCTGTCGAAATGGAG } \\
\text { GTGTCTTCCATGCCAACCTT }\end{array}$ & $\begin{array}{l}\text { Colo } \\
320 \\
\text { HSR }\end{array}$ & this thesis & Qiagen \\
\hline
\end{tabular}




\section{III.11.2 Synthetic DNA-oligonucleotides for cloning}

The DNA-oligonucleotides listed in Table 12 were used for the cloning of a mWif1 expression vector.

Table 12: Oligonucleotides for cloning

1: Designation of synthetic DNA-oligonucleotide; 2: nucleotide sequence of oligonucleotides $\left(5^{\prime} \rightarrow 3^{\prime}\right)$; 3 : Reference.

\begin{tabular}{l|l|l}
\hline 1 & 2 & 3 \\
\hline mWif1-BgllI-F & $\begin{array}{l}\text { AGTGCCAGATCTGCCGCCACCATGGCTCGG } \\
\text { AGAAGAGCCT }\end{array}$ & this thesis \\
\hline mWif1-Hpal-R & CGTTAACTCACCAGATGTAATTGGATTC & this thesis \\
\hline
\end{tabular}

\section{III.11.3 Synthetic DNA-oligonucleotides for sequencing}

The primers listed in Table 13 were used for sequencing of the $m$ Wif1 insert cloned into pMSCVpuro expression vector.

Table 13: Oligonucleotides for sequencing of $\boldsymbol{m W i f 1}$

1: Designation of oligonucleotide; 2: Nucleotide sequence of oligonucleotides; 3:Reference.

\begin{tabular}{l|l|l}
\hline 1 & 2 & 3 \\
\hline$m$ Wif1- $F$ & TCCTGTCAATATCCACTCCATGAA & König 2012 \\
\hline$m$ Wif1-intraF1 & AAGGAGACCTGTGCTCTAAGC & this thesis \\
\hline$m$ Wif1- $R$ & CTGATGCCTTGTGAGGCACT & König 2012 \\
\hline$m$ Wif1-seq-rv1 & CCTCCATTTCGGCAGGGTTGG & this thesis \\
\hline$m W$ if1-tq- $F$ & CGCCCATCAGGCTAGAGTGC & Ecke 2008 \\
\hline$m W$ if1-tq- $R$ & GACAGGAATGGCTGGCATTCT & Ecke 2008 \\
\hline
\end{tabular}

\section{III.11.4 Synthetic DNA-oligonucleotides for genotyping}

Table 14: Oligonucleotides for genotyping

1: Designation of analyzed allele; 2: Designation of oligonucleotide nucleotide; 3: Sequence of oligonucleotides; 4: Reference.

\begin{tabular}{l|l|l|l}
\hline 1 & 2 & 3 & 4 \\
\hline Ptch $w t$ & $\begin{array}{l}\text { mPTCNx_f } \\
\text { mPTCwt_r.2 }\end{array}$ & $\begin{array}{l}\text { TGGTAATTCTGGGCTCCCGT } \\
\text { ACACAACAGGGTGGAGACCACT }\end{array}$ & (Uhmann et al. 2007) \\
\hline Ptch flox & $\begin{array}{l}\text { mPTCNx_f } \\
\text { mPTCNx_r }\end{array}$ & $\begin{array}{l}\text { TGGTAATTCTGGGCTCCCGT } \\
\text { CCGGTAGAATTAGCTTGAAGTTCCT }\end{array}$ & (Uhmann et al. 2007) \\
\hline
\end{tabular}




\begin{tabular}{l|l|l|l}
\hline Ptch del & $\begin{array}{l}\text { Exon7-F } \\
\text { neo-R }\end{array}$ & $\begin{array}{l}\text { AGGAAGTATATGCATTGGCAGGAG } \\
\text { GCATCAGAGCAGCCGATTGTCTG }\end{array}$ & (Uhmann et al. 2007) \\
\hline \multirow{2}{*}{ CreERT2 } & $\begin{array}{l}\text { Ella-Cre-F } \\
\text { Ella-Cre-R }\end{array}$ & $\begin{array}{l}\text { CCAGGCTAAGTGCCTTCTCTACA } \\
\text { AATGCTTCTGTCCGTTTGCCGGT }\end{array}$ & (Uhmann et al. 2007) \\
\hline \multirow{2}{*}{ Wif1 wt } & $\begin{array}{l}\text { mWif1-geno-F } \\
\text { mWif1-wt-R }\end{array}$ & $\begin{array}{l}\text { CGAGAACTTCACAAGCAGCACAGG } \\
\text { CCTGTTACAAATCTGCAGTCAGGA }\end{array}$ & (Kansara et al. 2009) \\
\hline $\begin{array}{l}\text { Wif1 } \\
\text { knockout } \\
\text { allele }\end{array}$ & $\begin{array}{l}\text { mWif1-geno-F } \\
\text { mWif1-TauR2 }\end{array}$ & $\begin{array}{l}\text { CGAGAACTTCACAAGCAGCACAGG } \\
\text { CTGGATCCGGGTGGCGTTGGC }\end{array}$ & (Kansara et al. 2009) \\
\hline
\end{tabular}

\section{III.12 Enzymes}

All enzymes were used with appropriate buffers and according to manufacturer`s protocol.

Table 15: Enzymes

\begin{tabular}{l|l}
\hline Enzyme & Supplier \\
\hline BgllI & Invitrogen GmbH, Karlsruhe \\
\hline HindIII & Invitrogen GmbH, Karlsruhe \\
\hline DNase I & Qiagen GmbH, Hilden \\
\hline Phusion DNA Polymerase & New England Biolabs GmbH, Frankfurt am Main \\
\hline Proteinase K & Carl Roth GmbH \& Co. KG, Karlsruhe \\
\hline RNase A & Carl Roth GmbH \& Co. KG, Karlsruhe \\
\hline RQ1-DNase & Promega GmbH, Mannheim \\
\hline SuperScriptll Reverse Transcriptase & Invitrogen GmbH, Karlsruhe \\
\hline T4 DNA Ligase & Invitrogen GmbH, Karlsruhe \\
\hline Taq DNA Polymerase & Molzym GmbH Co.KG, Bremen \\
\hline
\end{tabular}




\section{III.13 Antibodies}

\section{III.13.1 Primary antibodies}

Table 16: Primary antibodies for immunohistochemistry

1: Designation; 2: Source; 3: Dilution; 4: Antigen retrieval; 5: Supplier.

\begin{tabular}{|c|c|c|c|c|}
\hline 1 & 2 & 3 & 4 & 5 \\
\hline$\beta$-catenin (610153) & $\begin{array}{l}\text { mouse, } \\
\text { polyclonal }\end{array}$ & $1: 100$ & $\begin{array}{l}\mathrm{TE}, \mathrm{pH} 9.0 \\
\text { microwave }\end{array}$ & Becton Dickinson $\mathrm{GmbH}$, Heidelberg \\
\hline $\begin{array}{l}\text { CD34 antibody } \\
\text { (MEC14.7) }\end{array}$ & $\begin{array}{l}\text { rat, } \\
\text { polyclonal }\end{array}$ & $1: 1000$ & $\begin{array}{l}\text { boric acid, } \mathrm{pH} 5.1 \text {, } \\
30 \min 60^{\circ} \mathrm{C}\end{array}$ & Bio-Rad Laboratories GmbH, München \\
\hline E-cadherin (610181) & $\begin{array}{l}\text { mouse, } \\
\text { polyclonal }\end{array}$ & $1: 200$ & $\begin{array}{l}\mathrm{TE}, \mathrm{pH} 9.0 \\
\text { microwave }\end{array}$ & Becton Dickinson GmbH, Heidelberg \\
\hline $\begin{array}{l}\text { F4/80 antibody } \\
\text { Cl:A3-1 }\end{array}$ & $\begin{array}{l}\text { rat, } \\
\text { monoclonal }\end{array}$ & $1: 100$ & - & Bio-Rad Laboratories GmbH, München \\
\hline $\begin{array}{l}\text { Ki67 antibody } \\
\text { (556003) }\end{array}$ & $\begin{array}{l}\text { rabbit } \\
\text { monoclonal }\end{array}$ & $1: 50$ & $\begin{array}{l}\text { Citrate, } \mathrm{pH} 6.0 \text {, } \\
\text { microwave }\end{array}$ & Becton Dickinson $\mathrm{GmbH}$, Heidelberg \\
\hline $\begin{array}{l}\text { p53 Protein (CM5) } \\
\text { Antibody }\end{array}$ & $\begin{array}{l}\text { rabbit, } \\
\text { polyclonal }\end{array}$ & $1: 500$ & $\begin{array}{l}\mathrm{TE}, \mathrm{pH} 9.0 \\
\text { microwave }\end{array}$ & $\begin{array}{l}\text { Leica Biosystems Nussloch GmbH, } \\
\text { Nussloch }\end{array}$ \\
\hline Anti-Acta2 1A4 (Sma) & $\begin{array}{l}\text { mouse, } \\
\text { monoclonal }\end{array}$ & $1: 50$ & - & mous, monoclonal \\
\hline $\begin{array}{l}\text { Anti-WIF1 antibody } \\
\text { [EPR9385] } \\
(\text { ab155101) }\end{array}$ & $\begin{array}{l}\text { rabbit, } \\
\text { polyclonal }\end{array}$ & $1: 400$ & $\begin{array}{l}\mathrm{TE}, \mathrm{pH} 9.0 \text {, } \\
\text { microwave }\end{array}$ & Abcam plc., Cambridge, UK \\
\hline
\end{tabular}

Table 17: Primary antibodies for Western blot

\begin{tabular}{l|l|l|l}
\hline Designation & Source & Dilution & Supplier \\
\hline Akt (610861) & $\begin{array}{l}\text { mouse, } \\
\text { polyclonal }\end{array}$ & $1: 1000$ & Becton Dickinson GmbH, Heidelberg \\
\hline $\begin{array}{l}\text { Phospho-Akt (Ser473) } \\
\text { Antibody }\end{array}$ & $\begin{array}{l}\text { rabbit, } \\
\text { polyclonal }\end{array}$ & $1: 1000$ & $\begin{array}{l}\text { New England Biolabs GmbH, Frankfurt } \\
\text { am Main }\end{array}$ \\
\hline anti-Bcl-2, Clone: 6C8 & $\begin{array}{l}\text { rabbit, } \\
\text { polyclonal }\end{array}$ & $1: 200$ & Becton Dickinson GmbH, Heidelberg \\
\hline $\begin{array}{l}\text { B-catenin (610153) } \\
\text { mouse, } \\
\text { polyclonal }\end{array}$ & $1: 1000$ & Becton Dickinson GmbH, Heidelberg \\
\hline $\begin{array}{l}\text { Phospho-CaMKII } \\
\text { (Thr286) Antibody }\end{array}$ & $\begin{array}{l}\text { rabbit, } \\
\text { polyclonal }\end{array}$ & $1: 1000$ & $\begin{array}{l}\text { New England Biolabs GmbH, Frankfurt } \\
\text { am Main }\end{array}$ \\
\hline Caspase-3 & $\begin{array}{l}\text { rabbit, } \\
\text { polyclonal }\end{array}$ & $1: 1000$ & $\begin{array}{l}\text { New England Biolabs GmbH, Frankfurt } \\
\text { am Main }\end{array}$ \\
\hline cyclin E (M20) & $\begin{array}{l}\text { rabbit, } \\
\text { polyclonal }\end{array}$ & $1: 200$ & Santa Cruz Biotechnology, Inc. Heidelberg \\
\hline
\end{tabular}




\begin{tabular}{|c|c|c|c|}
\hline E-cadherin & $\begin{array}{l}\text { mouse, } \\
\text { polyclonal }\end{array}$ & $1: 2500$ & Becton Dickinson GmbH, Heidelberg \\
\hline $\begin{array}{l}\text { Phospho-SAPK/JNK } \\
\text { (Thr183/Tyr185) } \\
\text { Antibody }\end{array}$ & $\begin{array}{l}\text { rabbit, } \\
\text { polyclonal }\end{array}$ & $1: 1000$ & $\begin{array}{l}\text { New England Biolabs GmbH, Frankfurt } \\
\text { am Main }\end{array}$ \\
\hline $\begin{array}{l}\text { Phospho-c-Jun } \\
\text { (Ser63) II Antibody }\end{array}$ & $\begin{array}{l}\text { rabbit, } \\
\text { polyclonal }\end{array}$ & $1: 1000$ & $\begin{array}{l}\text { New England Biolabs GmbH, Frankfurt } \\
\text { am Main }\end{array}$ \\
\hline $\begin{array}{l}\text { Anti MAP Kinase } \\
\text { (ERK1, ERK2) } \\
\text { antibody }\end{array}$ & $\begin{array}{l}\text { rabbit, } \\
\text { polyclonal }\end{array}$ & $1: 1000$ & $\begin{array}{l}\text { Sigma Aldrich Chemistry } \mathrm{GmbH} \text {, } \\
\text { Steinheim }\end{array}$ \\
\hline $\begin{array}{l}\text { p44/42 MAPK (Erk1/2) } \\
\text { Antibody }\end{array}$ & $\begin{array}{l}\text { rabbit, } \\
\text { polyclonal }\end{array}$ & $1: 1000$ & $\begin{array}{l}\text { New England Biolabs GmbH, Frankfurt } \\
\text { am Main }\end{array}$ \\
\hline HSC 70 (B-6): sc-7298 & $\begin{array}{l}\text { mouse, } \\
\text { monoclonal }\end{array}$ & $1: 10000$ & Santa Cruz Biotechnology, Inc. Heidelberg \\
\hline $\begin{array}{l}\text { Phospho-PKC (pan) } \\
\text { (ßII Ser660) Antibody }\end{array}$ & $\begin{array}{l}\text { rabbit, } \\
\text { polyclonal }\end{array}$ & $1: 1000$ & $\begin{array}{l}\text { New England Biolabs GmbH, Frankfurt } \\
\text { am Main }\end{array}$ \\
\hline $\begin{array}{l}\text { S6 Ribosomal Protein } \\
\text { (54D2) }\end{array}$ & $\begin{array}{l}\text { mouse, } \\
\text { monoclonal }\end{array}$ & $1: 1000$ & $\begin{array}{l}\text { New England Biolabs GmbH, Frankfurt } \\
\text { am Main }\end{array}$ \\
\hline $\begin{array}{l}\text { Phospho-S6 } \\
\text { Ribosomal Protein } \\
\text { (Ser240/244) }\end{array}$ & $\begin{array}{l}\text { rabbit, } \\
\text { monoclonal }\end{array}$ & $1: 1000$ & $\begin{array}{l}\text { New England Biolabs GmbH, Frankfurt } \\
\text { am Main }\end{array}$ \\
\hline Anti-Acta2 1A4 (Sma) & $\begin{array}{l}\text { mouse, } \\
\text { monoclonal }\end{array}$ & $1: 50$ & Beckman Coulter Inc., Brea, USA \\
\hline Syndecan 2 antibody & $\begin{array}{l}\text { rabbit, } \\
\text { polyclonal }\end{array}$ & $1: 100$ & Biorbyt Ltd., Cambridge, UK \\
\hline $\begin{array}{l}\text { Anti-WIF1 antibody } \\
\text { [EPR9385] } \\
\text { (ab155101) }\end{array}$ & $\begin{array}{l}\text { rabbit, } \\
\text { polyclonal }\end{array}$ & $1: 1000$ & Abcam plc., Cambridge, UK \\
\hline
\end{tabular}

\section{III.13.2 Secondary antibodies}

Table 18: Secondary antibodies

1: Designation; 2: Source; 3: Specificity; 4: Application; 5: Supplier.

\begin{tabular}{l|l|l|l|l}
\hline $\mathbf{1}$ & $\mathbf{2}$ & $\mathbf{3}$ & $\mathbf{4}$ & $\mathbf{5}$ \\
\hline $\begin{array}{l}\text { ECL Anti-Mouse IgG, Horseradish Peroxidase- } \\
\text { Linked Species-Specific Whole Antibody }\end{array}$ & sheep & mouse & $\begin{array}{l}\text { Western } \\
\text { blot }\end{array}$ & $\begin{array}{l}\text { Amersham Biosciences } \\
\text { Europe GmbH, Freiburg }\end{array}$ \\
\hline $\begin{array}{l}\text { Anti-rabbit IgG (whole molecule) peroxidise } \\
\text { conjugate }\end{array}$ & goat & rabbit & $\begin{array}{l}\text { Western } \\
\text { blot }\end{array}$ & $\begin{array}{l}\text { Sigma Aldrich Chemistry } \\
\text { GmbH, Steinheim }\end{array}$ \\
\hline \begin{tabular}{l} 
EnVisionTM/HRP, Rabbit/Mouse (ENV) \\
\hline
\end{tabular} & goat & $\begin{array}{l}\text { rabbit, } \\
\text { mouse }\end{array}$ & IHC & Dako GmbH, Hamburg \\
\hline
\end{tabular}




\begin{tabular}{l|l|l|l|l}
\hline Anti-Rabbit Immunoglobulins/AP (D 0487) & goat & rabbit & IHC & Dako GmbH, Hamburg \\
\hline $\begin{array}{l}\text { donkey anti-goat IgG (H+L) (clone: pAK)-HRPO } \\
\text { Biotinylated Rabbit Anti-Rat IgG Antibody }\end{array}$ & donkey & goat & IHC & $\begin{array}{l}\text { Dianova GmbH, } \\
\text { Hamburg }\end{array}$ \\
\hline $\begin{array}{l}\text { (BA-4001) } \\
\text { Streptavidin/HRP (P0397) }\end{array}$ & rabbit & rat & IHC & $\begin{array}{l}\text { Biozol Diagnostica } \\
\text { Vertrieb GmbH, Eching }\end{array}$ \\
\hline
\end{tabular}

\section{III.14 Molecular biological methods}

All subsequently described methods are standard procedures that have been described elsewhere.

\section{III.14.1 Isolation of nucleic acids}

\section{III.14.1.1 Isolation of plasmid DNA from bacteria}

For small- and medium-scale isolation of plasmid DNA from bacteria the HiPure Plasmid DNA Purification Kit was used according to manufacturer's instructions.

\section{III.14.1.2 Isopropanol/ethanol precipitation of nucleic acids}

2 volumes of $100 \%$ ethanol or alternatively an equal volume of isopropanol were added to the nucleic acid solution, thoroughly mixed and precipitated overnight at $-20^{\circ} \mathrm{C}$. Then, solution was centrifuged for $30 \mathrm{~min}$ at $16000 \times \mathrm{g}$ and $4^{\circ} \mathrm{C}$. The pellet was washed twice with $70 \% \mathrm{EtOH}$ for $10 \mathrm{~min}, 4^{\circ} \mathrm{C}$ and $16000 \times \mathrm{g}$, air dried and dissolved in DNase/RNase-free water and the DNA/RNA concentration was measured via photometer (Eppendorf). 


\section{III.14.1.3 Isolation of total RNA from embryonic tissue}

To isolate total RNA from embryos tissue was minced with a scalpel blade, homogenized $60 \mathrm{sec}$ in $1 \mathrm{ml}$ Trizol on ice, incubated for $5 \mathrm{~min}$ at room temperature $(\mathrm{RT})$ and mixed with $200 \mu \mathrm{l}$ chloroform. The mixtures were vortexed, incubated for $3 \mathrm{~min}$ at RT and centrifuged for $10 \mathrm{~min}$ at $4^{\circ} \mathrm{C}$ and $800 \times$ g. Afterwards, the upper phase was precipitated with $500 \mu \mathrm{l}$ isopropanol overnight at $-20^{\circ} \mathrm{C}$ and centrifuged for $30 \mathrm{~min}$ at $16000 \times \mathrm{g}$ and $4^{\circ} \mathrm{C}$. The pellet was washed twice with $70 \% \mathrm{EtOH}$ for $10 \mathrm{~min}, 4^{\circ} \mathrm{C}$ and $16000 \times \mathrm{g}$, air dried and dissolved in RNase-free water and the RNA concentration was measured via photometer (Eppendorf).

\section{III.14.1.4 Isolation of total RNA from murine tissue}

RNA from skin samples was isolated using the RNeasy Fibrous Tissue Kit (Qiagen) following the manufacturer's instructions. Subsequently, the samples were incubated with DNase I (Qiagen). For this purpose, $10 \%(\mathrm{v} / \mathrm{v})$ RDD-buffer (supplied with DNase I) and $0.14 \mathrm{U} / \mu \mathrm{I}$ DNase I were added to the RNA, incubated $1 \mathrm{~h}$ at $37^{\circ} \mathrm{C}$ and then for $5 \mathrm{~min}$ at $65^{\circ} \mathrm{C}$. RNA was precipitated using $99 \% \mathrm{EtOH}$ overnight at $-20^{\circ} \mathrm{C}$, centrifuged at $16000 \times \mathrm{g}$ for $35 \mathrm{~min}$ and washed with $70 \% \mathrm{EtOH}$. The pellet was dissolved in $20 \mu \mathrm{l}$ RNase-free water and the RNA concentration was measured using a photometer (Eppendorf).

\section{III.14.1.5 Isolation of total RNA from cell culture}

Cells were rinsed with $1 \times$ PBS and detached in $1 \mathrm{ml}$ TRIzol by pipetting. To avoid RNA degradation the subsequent steps were performed on ice, unless stated otherwise. Samples were vortexed for $2 \mathrm{~min}$ and then incubated for 5 min at RT. Subsequently, $200 \mu$ of chloroform were added followed by vortexing for $15 \mathrm{sec}$ and an incubation step for $3 \mathrm{~min}$ at RT. Afterwards, the mixture was centrifuged for $10 \mathrm{~min}$ at $6000 \times \mathrm{g}$ and $4^{\circ} \mathrm{C}$. The upper phase 
containing the RNA was transferred into a new $1.5 \mathrm{ml}$ E-cup containing $1 \mathrm{ml}$ isopropyl alcohol, inverted and precipitated overnight at $-20^{\circ} \mathrm{C}$. Subsequently, the mixture was centrifuged for $30 \mathrm{~min}$ at $10000 \times \mathrm{g}$ and $4^{\circ} \mathrm{C}$. The supernatant was discarded and the remaining pellet was washed with $1 \mathrm{ml}$ $70 \% \mathrm{EtOH}\left(-20^{\circ} \mathrm{C}\right)$ by centrifugation for $10 \mathrm{~min}$ at $10000 \times \mathrm{g}$ and $4^{\circ} \mathrm{C}$. Afterwards, supernatant was removed and the pellet was air-dried at RT. Subsequently, the pellet was dissolved in $20 \mu \mathrm{l}$ Rase-free $\mathrm{H}_{2} \mathrm{O}$ for 5-10 min at $56^{\circ} \mathrm{C}$. The RNA concentration was measured using a photometer (Eppendorf).

\section{III.14.1.6 Laser microdissection}

For the microdissection $10 \mu \mathrm{m}$ paraffin sections were cut using a microtome, transferred to $\mathrm{MMI}$ membrane slides, dried for $30 \mathrm{~min}$ at $37^{\circ} \mathrm{C}$ and frozen at $80^{\circ} \mathrm{C}$. After thawing, sections were deparaffinized with xylene and rehydrated using descending ethanol series. To stain the nuclei the sections were incubated in hemalaun solution and $0.1 \% \mathrm{NaHCO}_{3}$ solution was added. After the staining reaction sections were dehydrated by ascending ethanol series and transferred in xylene. Laser microdissection was subsequently performed using MMI CellCut and MMI CellTools software and RNA was isolated with Arcturus Paradise PLUS FFPE RNA Isolation Kit according to the manufacturer`s protocol.

\section{III.14.1.7 Photometric quantification of nucleic acids}

$1 \mu \mathrm{l}$ of sample DNA or RNA was dissolved in $99 \mu \mathrm{l}$ water (1:100 dilution) and transferred into a cuvette. The concentration of nucleic acids was measured using a photometer at a wavelength of $260 \mathrm{~nm}$ to quantify the DNA/RNA and at a wavelength of $280 \mathrm{~nm}$ to determine the purity of the sample. Pure DNA or RNA sample have a 260/280 ratio of 1.8 and 2.0, respectively, and are relatively free from protein contamination. DNA or RNA concentrations can be estimated by measuring the absorbance at 260nm $\left(A_{260}\right)$, adjusting the 
$\mathrm{A}_{260}$ measurement for turbidity (measured by absorbance at $320 \mathrm{~nm}$ ), multiplying by the dilution factor, and using the relationship that an $A_{260}$ of 1.0 equates $50 \mu \mathrm{g} / \mathrm{ml}$ pure DNA or $A_{260}$ of 1.0 equates $40 \mu \mathrm{g} / \mathrm{ml}$ pure RNA. The concentration thus can be calculated according to the following formula:

concentration $(\mu \mathrm{g} / \mathrm{ml})=\left(\mathrm{A}_{260}\right.$ reading $-\mathrm{A}_{320}$ reading $) \times$ dilution factor $\times 50$ (or 40) $\mu \mathrm{g} / \mathrm{ml}$.

\section{III.14.2 Polymerase chain reaction}

\section{III.14.2.1 Reverse transcription}

Reverse transcription of RNA was performed in a final volume of $20 \mu \mathrm{l}$. $250 \mathrm{ng}$ hexamers were added to $2 \mu \mathrm{g}$ total RNA and the mixture was incubated for $10 \mathrm{~min}$ at $70^{\circ} \mathrm{C}$. Afterwards, $1 \times 1^{\text {st }}$ strand buffer (Invitrogen), $10 \mathrm{mM}$ DTT (Invitrogen) and 0.5mM dNTPs were added and the mixture was incubated at $25^{\circ} \mathrm{C}$ for $10 \mathrm{~min}$ and at $42^{\circ} \mathrm{C}$ for $2 \mathrm{~min}$. Subsequently, $1 \mu \mathrm{l}(200$ $\mathrm{U} / \mathrm{\mu l}$ ) SuperScript II Reverse Transcriptase (Invitrogen) was added and mixtures were incubated for $1 \mathrm{~h}$ at $42^{\circ} \mathrm{C}$, followed by incubation at $70^{\circ} \mathrm{C}$ for $10 \mathrm{~min}$. The efficiency of the reverse transcription is empirically $50 \%$, thus resulting in $1 \mu \mathrm{g} c D N A$ when $2 \mu \mathrm{g}$ RNA is reversely transcribed.

\section{III.14.2.2 Polymerase chain reaction of cDNA}

The amplification of cDNA in reaction volumes of $10-20 \mu$ l was performed with the following reagents and final concentrations: 
10-100 ng template-cDNA

$0.5 \mu \mathrm{M}$ sequence-specific forward-DNA-oligonucleotides

$0.5 \mu \mathrm{M}$ sequence-specific reverse-DNA-oligonucleotides

$0.2 \mathrm{mM}$ dNTP-mix

$1 \%(\mathrm{v} / \mathrm{v})$ N.N.-dimethyl sulfoxide (DMSO)

$10 \%(v / v)$ cresol solution

$1 \times$ polymerase-buffer

$0.1 \cup$ Taq-polymerase (Molzym)

PCR-conditions varied depending on utilized DNA-oligonucleotides and amplified fragment sizes. Usually the following conditions were applied:

$\begin{array}{ll}5 \mathrm{~min} & 95^{\circ} \mathrm{C} \\ 30 \mathrm{sec} & 95^{\circ} \mathrm{C} \\ 1 \mathrm{~min} & 58^{\circ} \mathrm{C} \\ 1 \mathrm{~min} & 72^{\circ} \mathrm{C} \\ 5 \mathrm{~min} & 72^{\circ} \mathrm{C} \\ \infty & 8^{\circ} \mathrm{C}\end{array} 27$ cycles

The results were visualized by agarose gel electrophoresis.

\section{III.14.2.3 Quantitative real-time PCR (qRT-PCR)}

The expression levels of different genes in allografts and BCCs were measured by means of qRT-PCR using SYBR Green (SYBR Green I, Invitrogen or QuantiTect SYBR Green, Qiagen). Genes analyzed were Axin2, c-myelocytomatosis oncogene (c-Myc), cadherin 1 and 2 (Cdh1 and 2), fibronectin 1 (Fn1), Glioma-associated oncogene family member 1 (Gli1), keratin 1 and 10 (K1 and K10), involucrin (IVI), loricrin (Lor), and Wnt inhibitory factor-1 (Wif1). The expression of these genes was measured using $5 \mathrm{ng}$ cDNA derived from allografts and human or murine BCC samples. For normalization the expression of the hypoxanthine guanine phosphoribosyl transferase (Hprt) and TATA box binding protein (Tbp) was measured in $5 \mathrm{ng}$ cDNA. Alternatively, the expression of $18 S$ rRNA in $16 \mathrm{pg}$ cDNA of each sample was measured. All oligonucleotides used are listed in 
Table 11. For qRT-PCR analyses SYBR Green from Invitrogen or Qiagen was used at the following conditions.

Qiagen: $\quad 50^{\circ} \mathrm{C}$ for $2 \mathrm{~min}$

$95^{\circ} \mathrm{C}$ for $15 \mathrm{~min}$

$\left.\begin{array}{l}95^{\circ} \mathrm{C} \text { for } 15 \mathrm{sec} \\ 60^{\circ} \mathrm{C} \text { for } 30 \mathrm{sec} \\ 72^{\circ} \mathrm{C} \text { for } 30 \mathrm{sec}\end{array}\right\} 40$ cycles

Invitrogen: $\quad 50^{\circ} \mathrm{C}$ for $2 \mathrm{~min}$

$94^{\circ} \mathrm{C}$ for $2 \mathrm{~min}$

$\left.\begin{array}{l}94^{\circ} \mathrm{C} \text { for } 15 \mathrm{sec} \\ 60^{\circ} \mathrm{C} \text { for } 1 \mathrm{~min}\end{array}\right\} 40$ cycles

For both reactions the following reagents were used in a total volume of $10 \mu \mathrm{l}:$

\section{$2.5 \mathrm{ng}$ template-cDNA}

$0.4 \mu \mathrm{M}$ sequence-specific forward-DNA-oligonucleotides

$0.4 \mu \mathrm{M}$ sequence-specific reverse-DNA-oligonucleotides

$1 \times$ SYBR Green master mix (containing HotStarTaq DNA Polymerase [Qiagen] or Platinum Taq DNA Polymerase [Invitrogen], SYBR Green PCR Buffer, dNTP mix, SYBR Green dye and ROX dye)

As data were analyzed by the standard curve method a standard curve with cDNA was compiled for each measurement. For this purpose, a series of seven 1:5 dilutions (S1-S7) was set up and amplified for each analysis. cDNA with a concentration of $10 \mathrm{ng} / \mu \mathrm{l}$ for $S 1$ from mouse BCCs, embryos isolated at either 13.5, 12.5 or 10.5 days post coitum (dpc) or HEK-293 cells treated with $\mathrm{LiCl}$ was used (see Table 11). The ABI Prism 7900HT Sequence Detection System was used for all qRT-PCR analyses. Each sample was measured in a reaction volume of $10 \mu \mathrm{l}$ in triplicates. 


\section{III.14.2.3.1 Data analysis using the standard curve method}

The results were analyzed using SDS 2.2.1 (Applied Biosystems) and Microsoft EXCEL (Microsoft Co) softwares. The standard was set up by a serial 1:5 dilution. For correct analysis the amplification curve ought to be in the exponential phase of the reaction when the Ct was achieved. Based on the determined values of the standard curve a standard line was compiled by plotting the logarithm of the quantity of cDNA against the Ct-value for each dilution. Thus, the standard line follows the equation $y=m x+b$. With this equation the expression level of each analyzed gene was determined in each sample. Each sample was normalized to the endogenous control (Hprt, Tbp, or $18 S$ rRNA, respectively).

\section{III.14.3 Cloning techniques}

\section{III.14.3.1 Restriction hydrolysis}

Restriction hydrolyses of DNA were performed in volumes of $10 \mu \mathrm{l}$ at $37^{\circ} \mathrm{C}$ for 2 h. $1 \mu \mathrm{g}$ DNA was hydrolyzed with 3 units $(U)$ of adequate restriction endonuclease. If possible, simultaneous hydrolysis of a DNA sample with two different enzymes was performed using a buffer ensuring optimal conditions for both enzymes. Alternatively, a serial restriction hydrolysis was performed. For this purpose, the DNA sample was first hydrolyzed for $1 \mathrm{~h}$ at $37^{\circ} \mathrm{C}$ using the first restriction endonuclease that has the lowest salt concentration in its recommended buffer. Subsequently, salt concentration was adjusted for the second reaction. Then, the second restriction endonuclease was added and incubated for $1 \mathrm{~h}$ at $37^{\circ} \mathrm{C}$. Restriction hydrolysis was stopped by heatinactivation if applicable. 


\section{III.14.3.2 Isolation of DNA fragments from agarose gels}

DNA fragments from agarose gels were excised under UV-light using a sterile scalpel blade and were extracted from the gel using the High Pure PCR Cleanup Micro Kit according to the manufacturer's instructions.

\section{III.14.3.3 Ligation}

Ligation of DNA fragments was performed using T4 DNA Ligase as it is capable of catalyzing the ligation of two DNA double-strands in the presence of ATP between the $5^{\prime}$-phosphate and the 3 '-hydroxyl groups of adjacent nucleotides in either a cohesive-ended or blunt-ended configuration. Ligation was conducted using 1 x ligation buffer and 4 U T4 DNA ligase (Invitrogen) in a total reaction volume of $20 \mu \mathrm{l}$. The amount of insert per ng of vector used was estimated from the gel and calculated according to the formula:

$$
\mathrm{ng} \text { of insert } \approx \frac{\text { (length of insert }) \times(\mathrm{ng} \text { of vector })}{\text { (length of vector })} \times \text { molar ratio }\left(\frac{\text { insert }}{\text { vector }}\right)
$$

The molar ratio of insert to vector (100-200 ng) was set 3 to 1.

\section{III.14.3.4 DNA-sequencing}

For cycle-sequencing of pMSCVpuro-mWif1 $500 \mathrm{ng}$ of the plasmid, $1 \mu \mathrm{M}$ primer (see Table 13), 1 x Sequencing Buffer, $1 \mu$ l BigDye ${ }^{\circledR}$ Terminator v3.1 Ready Reaction Mix were used in a total reaction volume of $10 \mu \mathrm{l}$. The following cycler program was used:

\begin{tabular}{|c|c|}
\hline $1 \mathrm{~min}$ & $95^{\circ} \mathrm{C}$ \\
\hline $30 \mathrm{sec}$ & $95^{\circ} \mathrm{C}$ \\
\hline $2.5 \mathrm{~min}$ & $60^{\circ} \mathrm{C}$ \\
\hline $5 \mathrm{~min}$ & $60^{\circ} \mathrm{C}$ \\
\hline$\infty$ & $10^{\circ} \mathrm{C}$ \\
\hline
\end{tabular}

Amplificates were purified using Sephadex G-50 in a MultiScreen ${ }_{H T S}-H V$ plate and the analysis of the sequence was performed on a $\mathrm{ABI} 3500 \mathrm{XL}$ 
genetic analyzer and $\mathrm{ABI} 3500$ data collection software. Further analysis was performed with the Sequencing Analysis Software v5.4 and BioEdit software.

\section{III.14.3.5 Cloning of a Wif1 expression plasmid}

A Wif1 expression plasmid was generated by inserting the mWif1 cDNA amplified from pcDNA3-mWif1 (Becker 2011) in the vector backbone pMSCVpuro.

At first, the mWif1 insert was generated by means of PCR using a forward primer containing a Bgll recognition sequence and a Kozak consensus sequence, and a reverse primer containing an Hpal recognition sequence (see Table 12). Integrity of the amplicon was determined by gel electrophoresis. A band of 1156 bp was generated as expected and purified from the agarose gel (see section III.14.3.2 and Fig. 5).

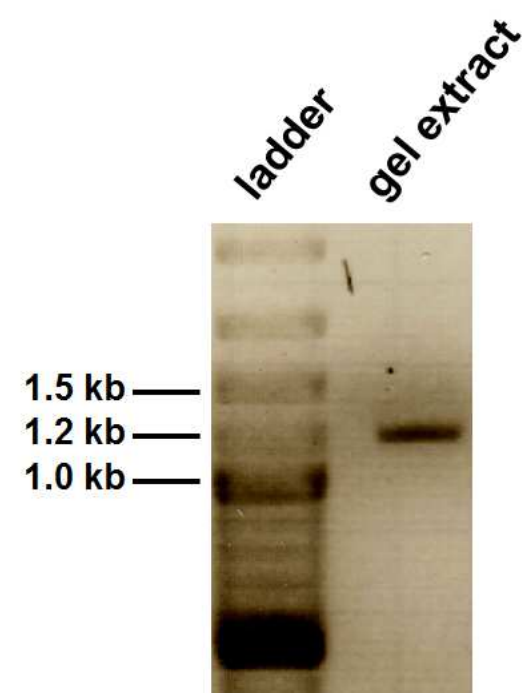

Fig. 5: Gel electrophoresis of amplified and purified Wif1 insert

Insert is 1156 bp long containing Bgll recognition sequence, a Kozak consensus sequence, the 1140 bp coding sequence and an Hpal recognition sequence.

Then, insert and $p M S C V$ puro were hydrolyzed with restriction endonulceases $B g / l$ and Hpal, purified by ethanol precipitation (see II.14.X) and dissolved in $20 \mu \mathrm{ldd} \mathrm{d}_{2} \mathrm{O} .2 \mu \mathrm{l}$ of each $20 \mu \mathrm{l}$ DNA solution was analyzed by gel electrophoresis (see Fig. 6). The concentration of DNA solutions was estimated by comparing the respective band intensity with band intensity of neighboring DNA ladder having a known DNA concentration. 


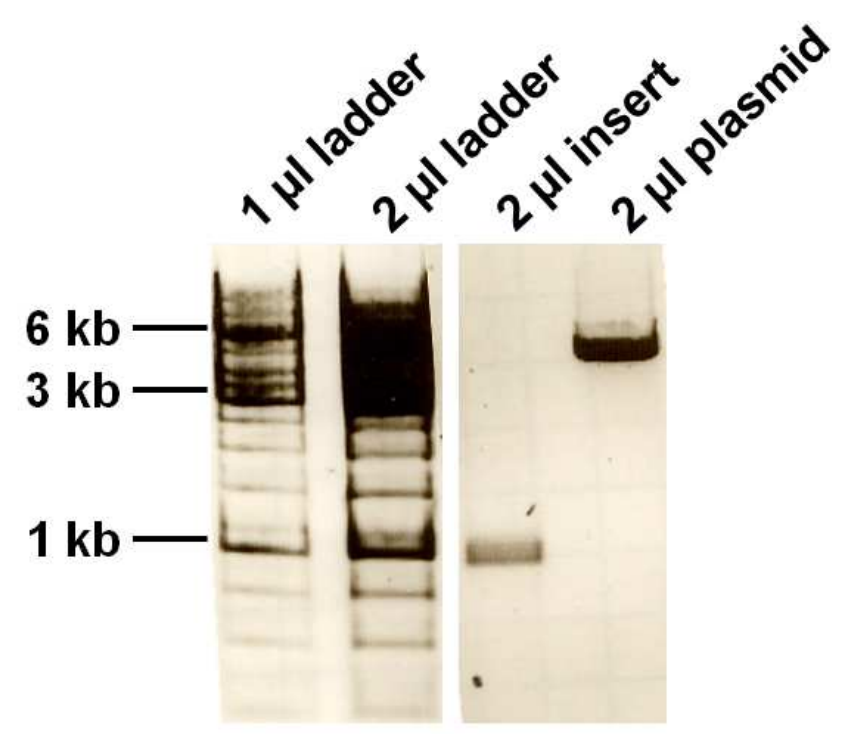

Fig. 6: Gel electrophoresis of hydrolyzed Wif1 insert and $p M S C V$ vector backbone

Insert is 1156 bp long containing Bglll recognition sequence, a Kozak consensus sequence, the 1140 bp coding sequence and an Hpal recognition sequence. $p M S C V$ vector backbone has a given size of $6.3 \mathrm{~kb}$.

Subsequently, a 3:1 ratio of the insert to vector DNA was used for ligation (see III.14.3.3) and transformed into E. coli (see III.14.4.1). Single cell clones were picked for small-scale isolation of plasmid DNA from bacteria culture (see III.14.1.1). A total number of 18 clones were isolated. Successful cloning of pMSCVpuro-Wif1 was verified by restriction hydrolyses (see III.14.3.1) and DNA sequencing (see III.14.3.4) (see Fig.7 and data not shown). By using $B g / l l$ and $H$ pal only plasmids containing the full-length insert are hydrolyzed resulting in a $1.2 \mathrm{~kb}$ (mWif1-insert) and a $6.3 \mathrm{~kb}$ (pMSCVpuro) fragment (top panel). Using HindllI and BamHI the orientation of the inserts was determined. In a $5^{\prime} \rightarrow 3^{\prime}$ orientation two fragments of $560 \mathrm{bp}$ and $6.9 \mathrm{~kb}$ length were generated. When inserts were in $3^{\prime} \rightarrow 5^{\prime}$ orientation restriction hydrolysis would result in fragments of 1.6 and $5.7 \mathrm{~kb}$ length (bottom panel). 


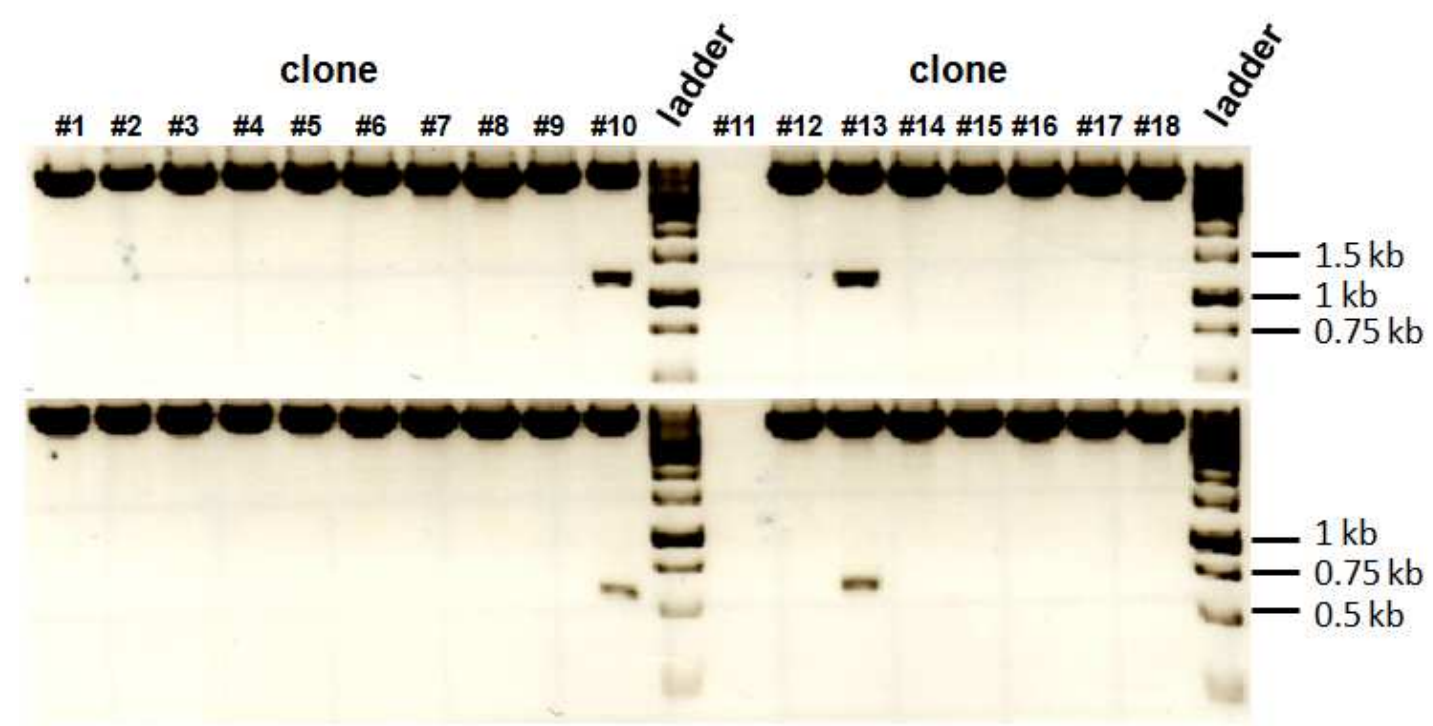

Fig. 7: Gel electrophoresis of restriction hydrolyzed pMSCV-Wif1

18 clones were picked from agar plates for downstream analysis. Top: Use of restriction enzymes Bg/ll and Hpal result in the generation of a $1.2 \mathrm{~kb}$ and a $6.3 \mathrm{bp}$ fragment. Bottom: An insertion in $5{ }^{\prime} \rightarrow 3^{\prime}$ orientation resulted in two fragments of $560 \mathrm{bp}$ and $6.9 \mathrm{~kb}$ length when hydrolyzed with Hindll and BamHI. A 3' $\rightarrow 5$ ' orientation would result in fragments of 1.6 and $5.7 \mathrm{~kb}$ length.

The 2 clones $\# 10$ and $\# 13$ were shown to have the insert in the correct orientation. All following experiments were performed with plasmid derived from clone \#10. The plasmid pMSCVpuro-mWif1 (hereafter pMSCV-Wif1) was transfected in the packaging cell line Platinum-E for the production of retroviral particles (see III.14.4.8).

\section{III.14.4 Cell biological techniques}

\section{III.14.4.1 Transformation of bacteria}

$100 \mathrm{ng}$ plasmid was added to $50 \mu \mathrm{l}$ competent cells (DH5a) and incubated for $20 \mathrm{~min}$ on ice. Afterwards, bacteria were transformed by heat-shock for 45 sec at $42^{\circ} \mathrm{C}$ and chilled on ice for $2 \mathrm{~min}$. Subsequently, $500 \mu \mathrm{l} \mathrm{SOC}$-medium were added and cells were incubated for $1 \mathrm{~h}$ at $37^{\circ} \mathrm{C}$ and $900 \mathrm{rpm}$ on a heating block. $50 \mu \mathrm{l}$ of the bacterial solution were plated on an agarose plate containing adequate antibiotics $(1 \mu \mathrm{g} / \mathrm{ml})$ and incubated overnight $(\mathrm{O} / \mathrm{N})$ at $37^{\circ} \mathrm{C}$. 


\section{III.14.4.2 Culture of eukaryotic cells}

Cells were cultured in an incubator at $5 \% \mathrm{CO}_{2}, 90 \%$ humidity and $37^{\circ} \mathrm{C}$. Medium was changed every three days and cells were subcultured by splitting them $1: 10$ upon reaching $70-80 \%$ confluence. Culture of cells was performed using media listed in Table 8.

\section{III.14.4.3 Isolation and cultivation of BMDMs}

To isolate bone marrow-derived macrophages (BMDMs) the femur of a wt mouse was excised and the attached connective and muscle tissue was removed. Both ends of the femur were cut by scissors and macrophages of the bone marrow were expelled in a petri dish using a syringe and $10 \mathrm{ml}$ Pluznik medium (DMEM/10 \% FCS/5 \% horse serum/30 \% L929 CM/1 \% $\mathrm{P} / \mathrm{S} / 0.0002 \% \beta$-mercaptoethanol). L929 CM was prepared as described in section II.14.4.4.2. Afterwards, cells were incubated $\mathrm{O} / \mathrm{N}$ at $37^{\circ} \mathrm{C}, 5 \% \mathrm{CO} 2$ and $95 \%$ humidity. The Supernatant containing the unattached macrophages was centrifuged for $10 \mathrm{~min}$ at $800 \times \mathrm{g}$, the cell pellet was dissolved in $40 \mathrm{ml}$ Pluznik medium, split and subcultivated in 4 uncoated petri dishes. The culture medium was changed every third day. 7 days after the isolation of BMDMs, cells were detached from petri dishes using Accutase and used for coculture proliferation assay (see section III. 14.4.10.2)

\section{III.14.4.4 Conditioned media (CM)}

\section{III.14.4.4.1 Generation and use of Wnt3a and Wnt5a CM}

$\mathrm{NIH} / 3 \mathrm{~T} 3 \mathrm{Wnt5a}, \mathrm{NIH} / 3 \mathrm{~T} 3, \mathrm{~L}$ Wnt-3A and $\mathrm{L}$ cells were cultured as described in section III.14.4.2. Cells that were $70 \%$ confluent were subcultured by splitting them 1:10 in media without selective antibiotics. After 4 days the first fraction of the conditioned medium was collected and new medium was added for additional 3 days for the production of the second fraction. The 2 conditioned media fractions were combined and centrifuged at $1000 \times \mathrm{g}$ for 5 
min to eliminate remaining cells and the supernatant was subsequently sterile filtered through $0.2 \mu \mathrm{m}$ filters and stored at $4^{\circ} \mathrm{C}$.

\section{III.14.4.4.2 Generation and use of L929 CM}

L929 conditioned medium was prepared from L929 cells having an initial $50 \%$ confluency and which were cultured 5 days in RPMI/10 \% FCS/1\% PS. Subsequently, the medium was collected and sterile filtered with a $0.2 \mu \mathrm{m}$ filter and stored at $-20^{\circ} \mathrm{C}$. L929 conditioned medium was used as indicated in section III.14.4.3

\section{III.14.4.5 Concentration of Wif1-containing media}

ASZ-pMSCV and ASZ-Wif1 cells were split 1:10 after reaching 70-80\% confluency and transferred in fresh culture medium without puromycin. After 5 days the medium was removed and replaced with fresh culture medium for 5 additional days. Both supernatants were subsequently centrifuged at $4500 \times \mathrm{g}$ for $10 \mathrm{~min}$ to remove all cells and debris. Afterwards, the supernatants were transferred to a protein concentrator tube (Thermo Fisher Scientific). Concentrator was used according to manufacturers' protocol generating a 75-fold concentrated supernatant.

\section{III.14.4.6 Transfection of eukaryotic cells}

HEK-293 cells were seeded in 6-well plates $\left(10^{5}\right.$ cells/well) in $1 \mathrm{ml}$ of the respective culture medium (see Table 8 ). $24 \mathrm{~h}$ later $1-2.2 \mu \mathrm{g}$ plasmid DNA and Roti-Fect (Carl Roth) transfection reagent (5 $\mu \mathrm{l}$ per $1 \mu \mathrm{g}$ DNA) were added to $100 \mu$ DMEM and incubated for $40 \mathrm{~min}$ at RT to allow for nucleic acid-lipid complex formation. Subsequently, the mixture was added to the cells cultured in $1 \mathrm{ml}$ of fresh culture medium without $\mathrm{P} / \mathrm{S}$ for $3 \mathrm{~h}$. Thereafter, the cells were washed and incubated with the respective culture medium for $24 \mathrm{~h}$. 


\section{III.14.4.7 Nucleofection of eukaryotic cells}

For nucleofection $2 \cdot 10^{6}$ ASZ001 cells were centrifuged at $90 \times \mathrm{g}$ for $10 \mathrm{~min}$. $2.5 \mu \mathrm{g}$ total plasmid (see scheme below) or $1 \mu \mathrm{g}$ nucleofection control plasmid (pGFPmax) in $100 \mu \mathrm{l}$ Nucleofector solution was added to the cell pellet and cells were resuspended. Then, cells were transferred into an electroporation cuvette and nucleofected using program T-029 in the electroporation device Amaxa Nucleofector 2b (Lonza). Afterwards, $100 \mu \mathrm{l}$ of nucleofected cells were directly plated in 96-well plates for downstream analysis via TOP/FOP reporter assay (see III.14.4.9).

Table 19: Scheme of plasmid combinations for nucleofection

$1: 1^{\text {st }}$ plasmid $(200 \mathrm{ng}) ; 2: 2^{\text {nd }}$ plasmid $(1 \mu \mathrm{g}) ; 3: 3^{\text {rd }}$ plasmid $(1 \mu \mathrm{g})$

\begin{tabular}{l|l|l}
\hline $\mathbf{1}$ & $\mathbf{2}$ & $\mathbf{3}$ \\
\hline$p R L-C M V$ & SuperTOPFlash (TOP) & $p C l-n e o-\beta$-catS33Y \\
\hline$p R L-C M V$ & SuperTOPFlash (TOP) & $p C R 3.1$ \\
\hline$p R L-C M V$ & SuperFOPFlash (FOP) & $p C l-n e o-\beta$-catS33Y \\
\hline$p R L-C M V$ & SuperFOPFlash (FOP) & $p C R 3.1$ \\
\hline- & pGFP-max & - \\
\hline
\end{tabular}

\section{III.14.4.8 Retroviral transduction of eukaryotic cells}

For preparation of virus particles used for transduction of ASZ001 cells Platinum-E cells were used as packaging cell line. Platinum-E cells were seeded at a confluency of $70 \%$ in $5 \mathrm{~cm}$ culture dishes one day before transfection with the retroviral vectors. For the transfection the following reagents were used:

$400 \mu$ l serum free medium (154 CF)

$9 \mu$ TransITß-LT1 Transfection Reagent

$3 \mu \mathrm{g}$ retroviral expression vector

(pMSCVpuro or pMSCVpuro-Wif1, respectively) 
The reagents were mixed and incubated at RT for 30 min. Afterwards, the Platinum-E cells were washed and the medium was replaced with $4 \mathrm{ml}$ fresh culture medium (154CF, 2\% FCS, 1\% P/S). The transfection mixture was added drop wise onto the plates and mixed by swirling. After $48 \mathrm{~h}$ the cell supernatants containing the viruses were sterile-filtered using a $0.45 \mu \mathrm{m}$ pore sterile filter. $3 \mathrm{ml}$ supernatant was mixed with $3 \mathrm{ml}$ fresh medium containing 3 $\mu \mathrm{g} / \mathrm{ml}$ polybrene and applied to a $5 \mathrm{~cm}$ culture dish containing $50 \%$ confluent ASZ001 cells. After $24 \mathrm{~h}$ the cells were washed and supplied with fresh medium. After additional $24 \mathrm{~h}$ the culture media were supplemented with puromycin.

\section{III.14.4.9 TOP/FOP reporter assay}

The $\beta$-catenin dependent activity of Wnt signaling was analyzed using a dual luciferase reporter assay. HEK-293 ells were seeded at a density of $1.5 \cdot 10^{5}$ cells/well in a 6-well plate. $24 \mathrm{~h}$ later cells were transfected with $1 \mu \mathrm{g}$ of either SuperTOPFlash (TOP) plasmid containing multiple $\mathrm{T}$ cell-specific transcription factor/lymphoid enhancer-binding factor (TCF/LEF)-binding sites or its negative control vector SuperFOPFlash (FOP) containing mutated and thereby inoperable binding sites $(1 \mu \mathrm{g}) .200 \mathrm{ng}$ of the renilla-luciferase encoding plasmid $p R L-C M V$ was cotransfected for normalization. Cotransfection with $1 \mu \mathrm{g} \mathrm{pCl}$-neo- $\beta$-catS33Y encoding for a constitutively active $\beta$-catenin served as positive control. In all settings, the final amount of DNA was increased to a total of $2.2 \mu \mathrm{g}$ with $p C R 3.1$ vector DNA. $24 \mathrm{~h}$ after transfection (see section III.14.4.6) cells were trypsinized and 6000 cells were subcultured in 96 well plates with Wnt3a $\mathrm{CM}$ or control medium. To block Wnt3a, Wnt3a CM was supplemented with $600 \mathrm{ng} / \mathrm{ml}$ Dkk1 or was preincubated with an equal volume of $75 \times$ concentrated ASZ-Wif1, ASZ-pMSCV (control) conditioned medium or with $30 \mu \mathrm{g} / \mathrm{ml} \mathrm{rWnt3a,}$ respectively, for $5 \mathrm{~h}$ at $\mathrm{RT}$ and $450 \mathrm{rpm}$ and then added to the transfected HEK-293 cells. After additional $48 \mathrm{~h}$, cells were lysed and renilla- and fireflyluciferase activity was measured using the Dual-Luciferase Reporter Assay 
System kit (Promega) according to manufacturer's protocol in a luminometer (Synergy Mx).

\section{III.14.4.10 Proliferation assay}

\section{III.14.4.10.1 5-Bromo-2'-deoxyuridine assay}

8000 cells were seeded in a 96 well plate $24 \mathrm{~h}$ before BrdU labeling. The BrdU labeling was performed for $24 \mathrm{~h}$ according to manufacturer's protocol (Roche).

The measurement was conducted by means of a plate reader (Synergy MX).

\section{III.14.4.10.2 Coculture proliferation assay}

On the first day $4 \times 10^{4}$ cells of the cell lines ASZ-pMSCV or ASZ-Wif1 were seeded in each well of a 24-well plate and $5 \times 10^{4}$ bone marrow derived macrophages (BMDMs), B9, NIH/3T3, NIH3T3-Wnt5a, Wnt-3A or L cells were seeded in 24-well inserts $(0.2 \mu \mathrm{m}$ pore size) placed in 24-well plates for coculture proliferation measurement. On each of 5 consecutive days the total cell number per well was counted using Celigo $S$ device and software.

\section{III.14.4.11 Cell cycle analysis}

For cell cycle analysis $1 \cdot 10^{6}$ cells were subcultivated in $10 \mathrm{~mm}$ culture dishes. After $48 \mathrm{~h}$ the cells were detached using Accutase and transferred into a $15 \mathrm{ml}$ tube containing $5 \mathrm{ml}$ Medium. Then, cells were washed by centrifugation for $5 \mathrm{~min}$ at $300 \mathrm{xg}$ and $4^{\circ} \mathrm{C}$ and resuspended in $1 \mathrm{ml}$ PBS. Subsequently, the cell suspension was mixed with $10 \mathrm{ml}-80^{\circ} \mathrm{C}$ $100 \%$ ethanol. Afterwards, the cells were fixed for $2 \mathrm{~h}$ at $-20^{\circ} \mathrm{C}$. Then, cells were centrifuged for $5 \mathrm{~min}$ at $1000 \times \mathrm{g}$ and $4^{\circ} \mathrm{C}$ and washed with $2 \mathrm{ml}$ $100 \%$ Ethanol by additional centrifugation for $5 \mathrm{~min}$ at $1000 \times \mathrm{g}$ and $4^{\circ} \mathrm{C}$. Subsequently, the cell pellet was resuspended in $500 \mu$ propidium iodide 
solution (10 $\mu \mathrm{g} / \mathrm{ml}$ propidium iodide and $100 \mu \mathrm{g} / \mathrm{ml}$ RNaseA in PBS) and incubated for $30 \mathrm{~min}$ at $37^{\circ} \mathrm{C}$. Afterwards, cells were analyzed using the flow cytometer LSR II (BD Biosciences) and FacsDiva ${ }^{\mathrm{TM}}$ (BD Biosciences) and FlowJo softwares (Tristar, Inc.), respectively.

\section{III.14.4.12 Cell viability assay}

8000 cells were seeded in a 96 well plate $24 \mathrm{~h}$ before adding WST-1. WST-1 was added for 4h. $200 \mathrm{mM} \mathrm{H} \mathrm{O}_{2}$ was added as negative control. The measurement was conducted by means of a plate reader (Synergy MX). Protocol and measurement was conducted according to manufacturer's protocol (Roche).

\section{III.14.4.13 Annexin V assay}

When in the early phase of apoptosis the integrity of the plasma membrane gets lost, phosphatidylserine translocates from the inner side of the plasma membrane to the cell surface. Annexin $\mathrm{V}$ is a $\mathrm{Ca}^{2+}$-dependent protein that can bind to PS. FITC coupled Annexin V was used to detect apoptosis and propidium iodide $(\mathrm{PI})$ was used to identify necrotic cells.

Cells were detached using $1 \mathrm{ml}$ Accutase and washed with $1 \times$ PBS by centrifugation at $300 \times \mathrm{g}$ for $5 \mathrm{~min}$. Afterwards, the cell pellet was resuspended in $100 \mu \mathrm{l} 1 \times$ Annexin V-binding buffer containing $2 \mu \mathrm{l}$ Annexin V-FITC for $10 \mathrm{~min}$. Subsequently, $2 \mu \mathrm{l}$ of a $100 \mu \mathrm{g} / \mathrm{ml} \mathrm{PI} \mathrm{solution}$ was added and cells were incubated in the dark at RT for $5 \mathrm{~min}$. Then, $400 \mu \mathrm{l}$ of $1 \times$ Annexin V-binding buffer were added and cells were analyzed using FACS Calibur (BD) and FlowJo software.

\section{III.14.4.14 Transwell migration assay}

75000 cells were seeded in 24-well inserts $(8.0 \mu \mathrm{m}$ pore size) and placed in a 24-well plate containing $500 \mu \mathrm{l}$ complete cell culture medium, Wnt3a CM or 
control CM. After $24 \mathrm{~h}$ migrated cells were stained with $5 \mu \mathrm{M}$ calcein for $1 \mathrm{~h}$. Subsequently, all cells that have not been migrated were removed and 5 consecutive pictures at 100 fold magnification were taken for each well. Cell number was counted using FIJI software.

\section{III.14.4.15 $\mathrm{Ca}^{2+}$-flux assay}

For measuring the intracellular levels of $\mathrm{Ca}^{2+}, 5 \times 10^{6}$ cells were incubated in $700 \mu \mathrm{L}$ RPMI 1640 medium supplemented with $5 \%$ FCS, 1 mM Indo-1-AM (Thermo Fisher Scientific), and $0.015 \%$ Pluronic F127 (Thermo Fisher Scientific) at $30^{\circ} \mathrm{C}$ for $25 \mathrm{~min}$. The cell suspension was diluted with $700 \mu \mathrm{L}$ RPMI 1640 containing $10 \%$ FCS and incubated for additional $10 \mathrm{~min}$ at $37^{\circ} \mathrm{C}$. Subsequently, cells were washed twice with $600 \mu \mathrm{L}$ Krebs Ringer solution. Prior to measurements cells were resuspended in $600 \mu \mathrm{L} \mathrm{Ca}^{2+}$-free Krebs Ringer solution supplemented with $0.5 \mathrm{mM}$ EGTA. Flow cytometric analysis was performed on an LSR II cytometer configured with a $488 \mathrm{~nm}$ and a $355 \mathrm{~nm}$ laser (BD Biosciences). After monitoring the basal $\mathrm{Ca}^{2+}$ level for $30 \mathrm{~s}$, cells were stimulated with $1 \mu \mathrm{l}$ ionomycin. The emission fluorescence intensities of Indo-1-AM were recorded at wavelengths of 405 $\mathrm{nm}$ and $530 \mathrm{~nm}$ to determine changes in the ratios of $\mathrm{Ca}^{2+}$-unbound versus $\mathrm{Ca}^{2+}$-bound Indo-1-AM. Data acquisition and analysis was performed with BD FacsDiva $^{\mathrm{TM}}$ (BD Biosiences) and FlowJo software (Tristar, Inc.), respectively.

\section{III.14.5 Western blot (WB)}

\section{III.14.5.1 Isolation of proteins from cell culture}

Cells were harvested in $1 \mathrm{ml}$ PBS using a cell scraper. Afterwards, cells were centrifuged for $5 \mathrm{~min}$ at $1000 \mathrm{xg}$ and $4^{\circ} \mathrm{C}$. The pellet was resuspended in $700 \mu \mathrm{l} \mathrm{PBS}$, centrifuged for $5 \mathrm{~min}$ at $1000 \times \mathrm{g}$ and $4^{\circ} \mathrm{C}$ and immediately shock-frozen in liquid nitrogen for cell lysis. Subsequently, the pellet was thawed on ice and resuspended in $50 \mu \mathrm{l}$ of modified RIPA buffer. After 30 $\min$ the lysate was centrifuged at $16000 \times \mathrm{g}$ and $4^{\circ} \mathrm{C}$ for $25 \mathrm{~min}$. The 
supernatant containing the proteins was stored at $-80^{\circ} \mathrm{C}$ and the protein concentration was measured with Pierce BCA Protein Assay Kit according to manufacturer's instructions.

\section{III.14.5.2 Isolation of proteins from tissue samples}

$30 \mathrm{mg}$ of tissue was cut with scalpel and added to $300 \mu$ modified RIPA buffer containing protease inhibitors in a $2 \mathrm{ml}$ reaction tube. Subsequently, the tissue was homogenized for $1 \mathrm{~min}$ at level B of homogenizer. Afterwards, homogenate was shock frozen in liquid nitrogen to disrupt cell membranes, thawed on ice and centrifuged at $10.000 \times \mathrm{g}$ for $10 \mathrm{~min}$ at $4^{\circ} \mathrm{C}$. Subsequently, the supernatant was transferred in a new $1.5 \mathrm{ml}$ reaction tube and the protein concentration was measured.

\section{III.14.5.3 Western blot}

For sample preparation $1 \times$ sample buffer was added to $35 \mu \mathrm{g}$ protein lysate and samples were filled up to equal volumes with lysis buffer. Proteins were denatured for $5 \mathrm{~min}$ at $96^{\circ} \mathrm{C}$ and $450 \mathrm{rpm}$ on a heating block. Proteins were separated using NuPAGE Novex Midi Gels in 1 x running buffer (NuPAGE MES SDS buffer, $20 \mathrm{x}$ ) for $1.5-2 \mathrm{~h}$ at $160 \mathrm{~mA}$. For the determination of the molecular weight $4 \mu \mathrm{l}$ SeeBlue Plus2 Prestained Standard were run in parallel. Proteins were transferred to a nitrocellulose membrane (GE Healthcare) using a semi-dry blotting device at $120 \mathrm{~mA}$ per gel for $80 \mathrm{~min}$. After blocking with $5 \%(\mathrm{w} / \mathrm{v})$ milk powder/TBST for $1.5 \mathrm{~h}$ at RT and washing three times for $10 \mathrm{~min}$ in TBST the primary antibody (see Table 17) was added in a dilution of 1:1000 in $0.02 \%(\mathrm{w} / \mathrm{v})$ sodium azide/3 \% (w/v) BSA in TBST. The membrane was incubated overnight on an orbital shaker at $4{ }^{\circ} \mathrm{C}$. Subsequently, the membrane was washed $3 \times 10 \mathrm{~min}$ in TBST and the secondary antibody $1: 5000$ in $5 \%(\mathrm{w} / \mathrm{v})$ milk powder/TBST was added for $1 \mathrm{~h}$ at RT on orbital shaker. After three washing steps in TBST $1.5 \mathrm{ml}$ the detection reagent (Amersham ECL Plus Western Blotting Detection 
Reagents) per membrane was added for $3 \mathrm{~min}$. For visualization the FluorchemQ device and AlphaView Software was used.

\section{III.14.6 Animal experiments}

\section{III.14.6.1 Mouse keeping}

All used mouse lines were kept within the institutes' own mouse facility at $12 \mathrm{~h}$ day-night-rhythm at $20 \pm 2^{\circ} \mathrm{C}$ and $50 \pm 10 \%$ humidity. Mice had ad libitum access to pelleted dry food and tap water. All experiments were performed in compliance with all relevant legal and ethical requirements. Immune deficient nude mice were kept in individually ventilated and sealed cages supplying ad libitum autoclaved pelletized dry food and tap water.

\section{III.14.6.2 Genotyping PCR}

Ear marks to identify each mouse and tail tip biopsies at the age of 3 weeks were performed by animal caretakers. Biopsies were digested using $0.5 \mathrm{mg} / \mathrm{ml}$ proteinase $\mathrm{K}$ in $400 \mu \mathrm{l} \mathrm{STE}$ buffer over night at $56^{\circ} \mathrm{C}$ followed by ethanol precipitation to isolate genomic DNA (gDNA). $1 \mu$ l of gDNA was used as template for genotyping PCR utilizing appropriate DNA-oligonucleotides listed in Table 14.

\section{III.14.6.3 Intramuscular tamoxifen injection}

Tamoxifen (Sigma-Aldrich Chemie $\mathrm{GmbH}$, Steinheim) was solved in $96 \%$ ethanol to obtain a $100 \mathrm{mg} / \mathrm{ml}$ tamoxifen solution which was diluted $1: 10$ in sterile sun flower oil (Sigma-Aldrich Chemie $\mathrm{GmbH}$, Steinheim). This emulsion $(10 \mathrm{mg} / \mathrm{ml})$ was stored at $-20^{\circ} \mathrm{C}$. For inducing the activity of Cre-recombinase in the mouse lines Ptch ${ }^{\text {floxflox }}$ CreERT2 ${ }^{T /}$ Wif $^{-1 /}$, Ptch $^{\text {floxflox }}$ Wif1 $^{-1-}$ and Ptch ${ }^{\text {floxfllox}}$ CreERT2 ${ }^{T /-} 10 \mu$ l of the tamoxifen emulsion 
was injected in the musculus soleus of 8 week old mice using BD microfine + Demi syringes (Zibat et al. 2009).

\section{III.14.6.4 BCC mouse model and Wif1 knockout}

The analysis of BCC development was conducted using Ptch $^{\text {floxflox }}$ ROSA26-CreERT2 ${ }^{\text {T/ }}$ mouse model (hereafter Ptch $^{\text {floxfllox }}{ }^{\text {CreERT2 }}{ }^{T /}$ ). In these mice tamoxifen injection can trigger the nuclear translocation of the ubiquitously expressed fusion protein consisting of a Cre-recombinase domain and a mutated estrogen receptor domain (CreERT2). Subsequently, nuclear CreERT2 can cause deletions at floxed sequences of host DNA.

To analyze the effect of Wif1 on BCC formation, development and regression $\mathrm{Ptch}^{\text {floxflox }}$ mice were crossed to $\mathrm{Wif}^{-1 /}$ mice (see Table 9). The resulting $\mathrm{Ptch}^{\mathrm{flox} /+}$ Wif $^{+/-}$were mated with to mice of the same gentype to generate $\mathrm{Ptch}^{\text {floxflox}}$ Wif $^{-1-}$ mice. These mice in turn were crossed with Ptch $^{\text {floxflox }}$ CreERT2 ${ }^{T /-}$ mice.

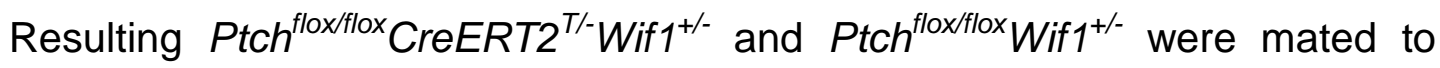
generate Ptch ${ }^{\text {floxflox }}$ CreERT2 ${ }^{T /}$ Wif $^{-1 /}, \quad$ Ptch $^{\text {floxfllox }}$ Wif $^{-1 /}$ and $\mathrm{Ptch}^{\text {floxflox}} \mathrm{CreERT2}^{\mathrm{T/}}$ which were injected with a single dose of $100 \mu \mathrm{l}$ tamoxifen emulsion (10 mg/ml, Sigma-Aldrich Chemie $\mathrm{GmbH}$, Steinheim) i.m. at an age of 8 weeks. For the genotypes Ptch ${ }^{\text {flox } f f l o x} C r e E R T 2^{T /-}$ Wif $1^{-1-}$ and Ptch $^{\text {floxflox }}$ CreERT2 ${ }^{T /-} 20$ animals were injected with tamoxifen. After 45 days and 90 days 10 animals each were euthanized for further analyses. For the genotype Ptch ${ }^{\text {flox flox }}$ Wiff $^{-1 /} 10$ animals were injected with tamoxifen. After 45 days and 90 days 5 animals each were euthanized for further analyses.

\section{III.14.6.5 Allograft}

Cells were suspended in a $200 \mu \mathrm{l}$ solution of $50 \%$ matrigel in complete cell culture medium without puromycin. Cell suspension was subsequently injected subcutaneously into the flanks of nude mice using $1 \mathrm{ml}$ Sub-Q 
syringes (BD) which were precooled on ice to prevent hardening of the matrigel. Each nude mouse was injected bilaterally on one flank with ASZ-pMSCV cell suspension and on the other flank with ASZ-Wif1 cell suspension to exclude mouse effects. After 2 weeks when the tumors reached a diameter of $5 \mathrm{~mm}$ the width (b) and length (a) of each tumor was measured by caliper 3 times a week and tumor volume $(\mathrm{V})$ was calculation by use of the modified ellipsoid formula: $V=\frac{1}{2} a \cdot b^{2}$ (Euhus et al. 1986; Tomayko and Reynolds 1989).

When the tumors reached a maximal diameter of $20 \mathrm{~mm}$ mice were euthanized and tumors were removed, weighed and measured. For RNA and protein isolation (see III.14.1.4 and III.14.6.2) portions of the tumors were snap-frozen on dry ice and for antibody stainings tumor portions were fixed in $4 \%$ PFA for 1 week in embedding cassettes.

\section{III.14.7 Histological stainings}

For all histological stainings, PFA-preserved tissue was embedded in paraffin, cut into $5 \mu \mathrm{m}$ sections and mounted onto glass slides. All samples were examined by a pathologist.

\section{III.14.7.1 Hematoxylin and eosin (H\&E) staining}

Sections were deparaffinized with xylene for $20 \mathrm{~min}$ and subsequently rehydrated using descending ethanol solutions. After washing with $\mathrm{ddH}_{2} \mathrm{O}$, slides were placed in hematoxylin solution for $15 \mathrm{~min}$. The staining reaction was done in warm tap water for at least $5 \mathrm{~min}$. Samples were then quickly dipped in $1 \%$ eosin solution containing $0.5 \%(\mathrm{v} / \mathrm{v})$ glacial acetic acid. Subsequently, slides were washed with $\mathrm{ddH}_{2} \mathrm{O}$ and dehydrated using ascending ethanol solutions. Slides were placed again in xylene and afterwards mounted in Pertex. Then, slides were dried at $55^{\circ} \mathrm{C}$ for $15 \mathrm{~min}$. 


\section{III.14.7.2 Immunohistochemistry (IHC)}

Sections were deparaffinized with xylene and rehydrated in descending ethanol series. Whenever necessary, antigen retrieval was done performed according to Table 16. Sections were blocked with $3 \% \mathrm{H}_{2} \mathrm{O}_{2}$ to inhibit endogenous peroxidases and blocked with $0.2 \%$ casein to prevent nonspecific antibody binding. Afterwards, the sections were incubated with a primary antibody over night at $4^{\circ} \mathrm{C}$ and on the next day with the appropriate secondary antibody (see Table 16 and Table 18) in a humid chamber. Antibody binding was visualized using DAB+ (Envision+ system-HRP, Dako) or aminoethylcarbazol as chromogen. To stop the reaction, slides were rinsed with distilled water. All sections were counterstained with hematoxylin.

\section{III.14.7.3 TdT-mediated dUTP-biotin nick end labeling (TUNEL)}

Tissue sections were deparaffinized with xylene and rehydrated with descending ethanol series. Afterwards, slides were first washed in $0.85 \%$ $\mathrm{NaCl}$ and then in $1 \times$ PBS. Subsequently, slides were fixated in 4\% PFA for 15 minutes at RT and washed twice in $1 \times$ PBS. Then, $20 \mu \mathrm{g} / \mathrm{ml}$ proteinase $\mathrm{K}$ solution was applied on each tissue section and incubated for 10 minutes at RT. Afterwards, slides were washed with $1 \times$ PBS. Tissue sections which were treated with a solution of 10 unit/ml RQ1 RNase-free DNase I in DNase I buffer served as positive controls. Then, sections were again fixated in $4 \%$ PFA for 5 min at RT and washed twice in 1x PBS. The labeling reaction was performed using the DeadEnd Colorimetric TUNEL System (Promega) according to the manufacturer's protocol. Subsequently, sections were counterstained with Hematoxylin and mounted using glycer gel (Dako). 


\section{III.14.7.4 Ladewig staining}

To stain for extra cellular matrix Ladewig staining was performed by the technical assistants in the department of neuropathology following standard protocols. Analysis was performed by means of FIJI software. 


\section{Results}

\section{IV.1 WIF1 expression in human BCC}

The relevance of WIF1 in human BCC was first tested by protein expression analysis of WIF1 on paraffin sections derived from 10 human BCC samples. As shown in Fig. 8 all analyzed human BCC samples showed WIF1 expression (10/10). In all samples WIF1 was homogeneously expressed by tumor cells (see Fig. 8, arrow head). It also was expressed by tumorassociated inflammatory cells (see Fig. 8, asterisk). Moreover, WIF1 expression in fibrobalsts of the tumor stroma was detectable (see Fig. 8, arrows). Together, these data show that WIF1 is highly expressed in BCC. Basically all tumor cells express this protein. WIF1 is also expressed in the tumor stroma, however to a variabel extend.
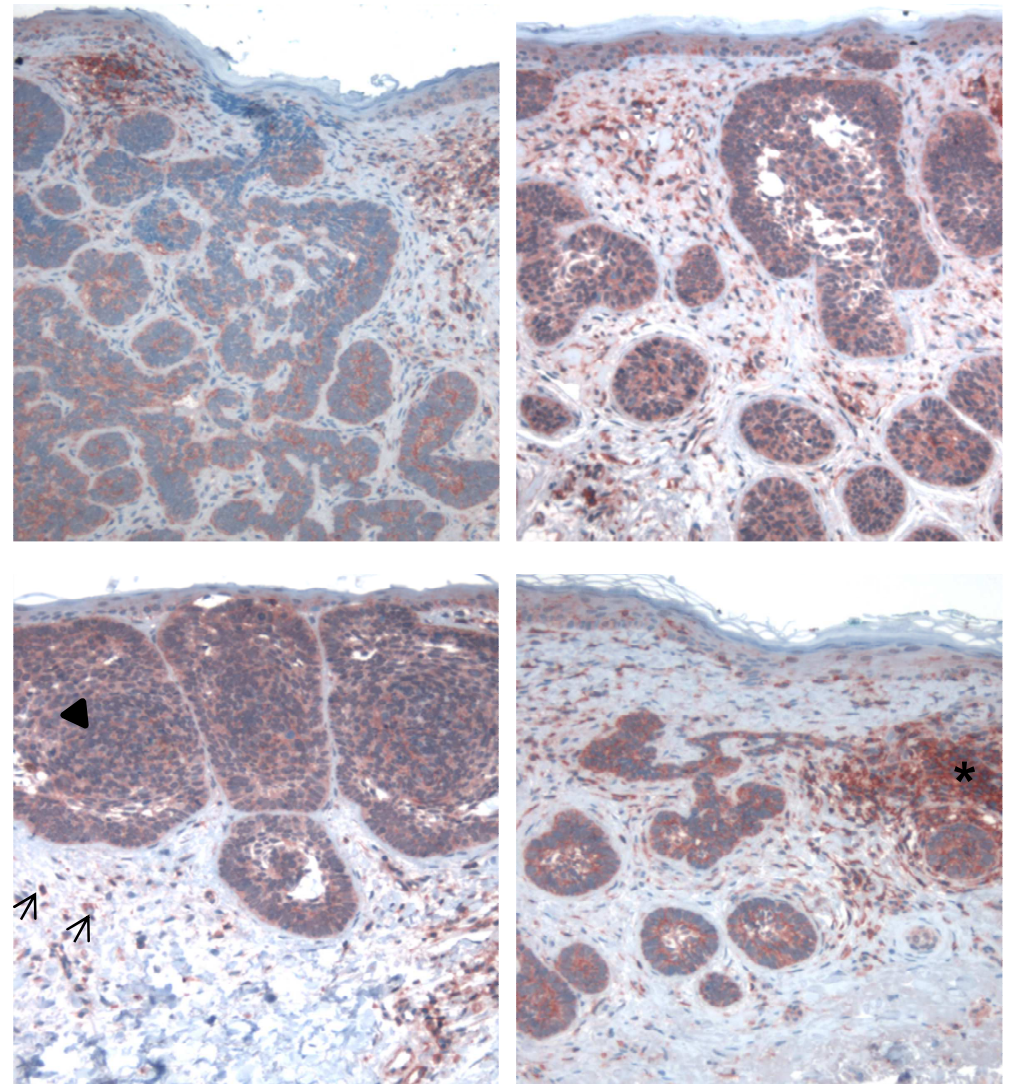

Fig. 8: Antibody staining of WIF1 in human BCC

Four representative stainings of paraffin sections derived from human BCC using an anti-WIF1 antibody. Staining reaction was performed using AEC. WIF1 expression is detectable in cells of inflammatory infiltrate (asterisk), tumor cells (arrow head), and stromal fibroblasts (arrows). 
As WIF1 is a well-known tumor suppressor we hypothesized that WIF1 expression might be absent or reduced in more aggressive skin tumors such as SCC and melanoma. In addition, we wanted to know whether there is any difference in WIF1 expression in subtypes of BCC. To test this, tumor tissue was separated from stromal cells by laser microdissection and WIF1 expression was analyzed in the tumor tissue by means of qRT-PCR. The analysis included 3 superficial, 3 nodular and 3 infiltrative BCC, 3 SCC and 3 melanomas. WIF1 was detectable in all samples (see Fig. 9). The weakest expression levels were detectable in superficial and nodular subtypes of BCC, the highest in infiltrative BCC, SCC and melanomas. No significant differences between infiltrative $\mathrm{BCC}, \mathrm{SCC}$ and melanomas were noticeable. Moreover, the differences between BCC subtypes were also not statistically significant. However, superficial and nodular BCC expressed significantly lower levels of WIF1 in comparison with SCC and melanomas. 


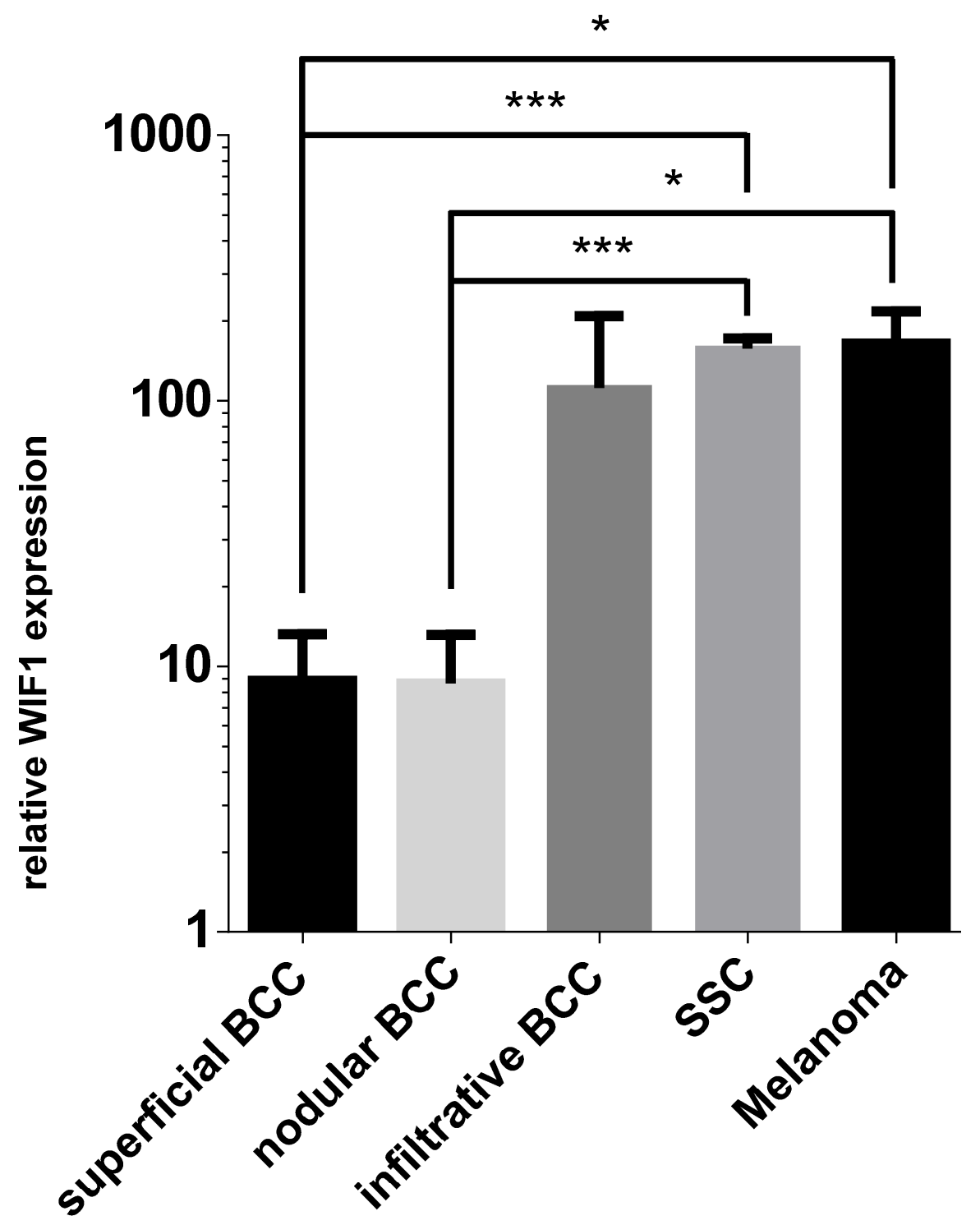

Fig. 9: Evaluation of WIF1 qRT-PCR of microdissected BCC subtypes, SCC and melanomas

3 skin samples each were analyzed. Tumor tissue was excised using laser microdissection. All data are represented as mean + SEM. Statistical significance was tested using unpaired t test with Welch`s correction. * $p<0.05$, ${ }^{* * *} p<0.001$.

Because these results seemed to be contradictory to the reported tumor suppressive function of WIF1, we analyzed the role of WIF1 in BCC in more detail. 


\section{IV.2 Generation of a Wif1 expression vector}

\section{IV.2.1 Cloning strategy}

According to CCDS database only one protein-coding Wif1 transcript is known. However, Ensemble database describes two distinct transcripts. These are a $1140 \mathrm{bp}$ transcript encoding for a 379 aa protein (see Fig. 10) and a transcript variant of $1098 \mathrm{bp}$ length encoding for a 365 aa protein lacking roughly the second half of exon 8 . Nevertheless, all investigations, at least to our knowledge, focused on the first one. To analyze the role of Wif1 in BCC formation and development we therefore also focused on this isoform.

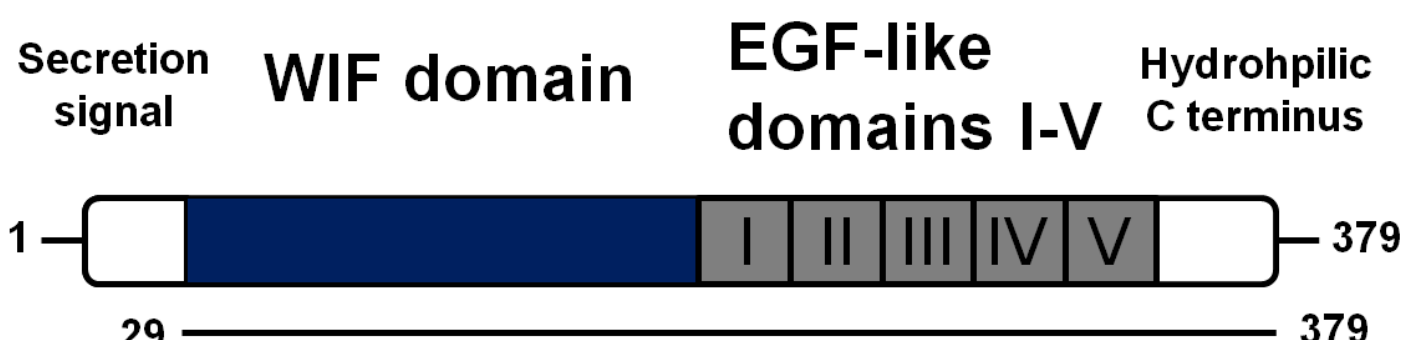

Fig. 10: Functional structure Wif1

Wif1 consists of a 28 amino acids long N-terminal secretion signal sequence which is cleaved off upon secretion, the WIF domain (WD, 143 amino acid residues), five EGF-like domains (31-33 residues each) and a hydrophilic C-terminus.

During my master thesis I cloned a Wif1 expression vector ( $p c D N A 3-m W i f 1$ ) containing the complete coding sequence of murine Wif1 together with a Kozak consensus sequence. However, as the BCC cell line ASZ001 is hard to transfect, the overexpression of Wif1 by means of conventional transfection of $p c D N A 3-m W i f 1$ was unsuccessful. Therefore, the insert was subcloned into pMSCVpuro to generate the retroviral expression vector pMSCV-Wif1 as described in III.14.3.5. 


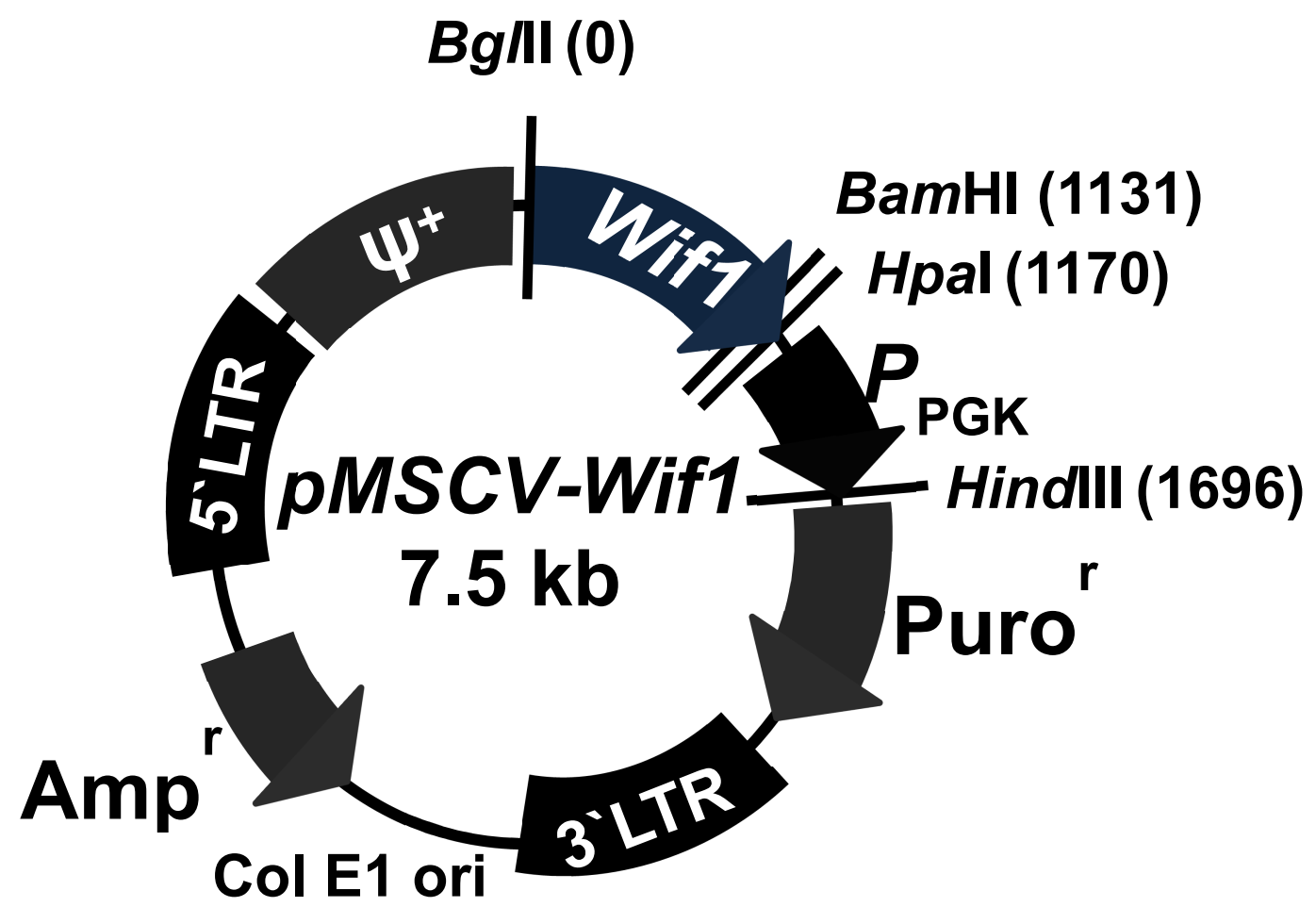

Fig. 11: Schematic of pMSCV-Wif1

Retroviral expression vector containing murine Wif1 ORF. Bgll(0), BamHI(1131), Hpal(1170), Hindll(1696): restriction sites for respective endonucleases. Puro': puromycin resistance cassette for stable selection in eukaryotic expression systems. Ampr: Ampicillin resistance cassette for selection in prokaryotic expression systems. $3^{`}$ and 5'LTR: $3^{\prime}$ and $5^{`}$ long terminal repeats, sequences allowing for stable integration into target cell genome. $\Psi^{+}$: packaging signal for retrovirus production in Platinum-E packaging cell line. $P_{\mathrm{PGK}}$ : promoter for puromycin expression in eukaryotic cells. Col E1 ori: origin of replication.

The correct orientation of the insert was assessed by restriction hydrolysis and sequencing. As described in III.14.4.8 the supernatants from Platinum-E cells with retroviral particles containing pMSCV (control) or pMSCV-Wif1, respectively, were used for transduction of ASZ001.

\section{IV.2.2 Selection of stably transfected cells and verification of Wif1 expression}

2 days after transduction of ASZ001 with either pMSCV control vector or the pMSCV-Wif1 overexpression plasmid, cells were treated with $2 \mu \mathrm{g} / \mathrm{ml}$ puromycin to select stably transduced cells. Moreover, single cell clones 
were isolated by limited dilution. RNA was isolated, reversely transcribed and a Wif1 specific PCR was performed (see Fig. 12)

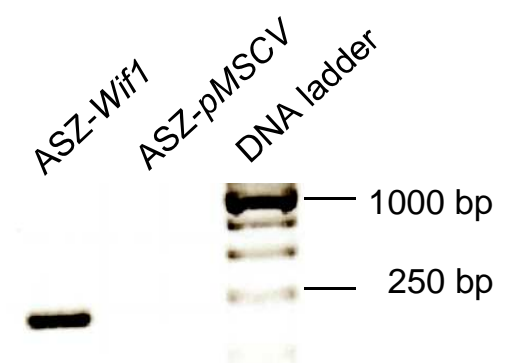

Fig. 12: PCR detecting Wif1 of pMSCV and pMSCV-Wif1 transduced ASZ001 cells PCR product of 220 bp length was generated as expected.

Moreover, in order to confirm the translation of the Wif1 mRNA a Western blot was performed (see Fig. 13).

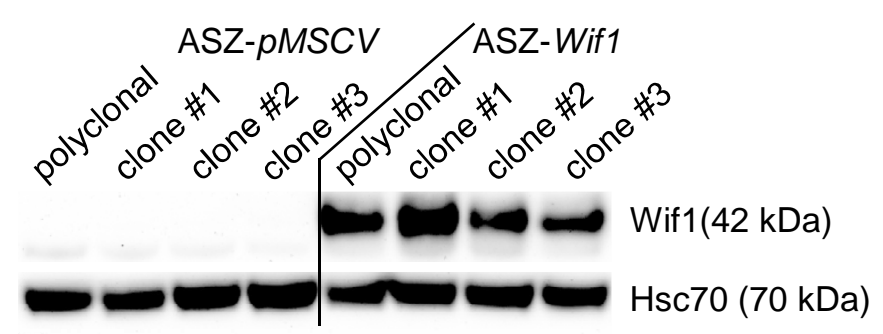

Fig. 13: Western blot detecting Wif1 of $p M S C V$ and pMSCV-Wif1 transduced ASZ001 cells Antibody detecting Hsc70 served as loading control. Single cell clones and a polyclonal cell population (no single cell clone selection) were analyzed regarding Wif1 expression.

As shown in Fig. 13 protein samples from pMSCV transduced ASZ001 cells (hereafter ASZ-pMSCV) do not express detectable levels of Wif1. In contrast, in the samples isolated from pMSCV-Wif1 transduced ASZ001 cells (hereafter ASZ-Wif1) the Wif1 protein was detected at the expected size of $42 \mathrm{kDa}$. This confirmed the successful transduction and operability of the retroviral expression plasmid pMSCV-Wif1 to induce Wif1 overexpression.

For the analysis of successful Wif1 production and secretion the supernatant of ASZ-Wif1 was 75-fold concentrated (see III.14.4.5) and analyzed by Western blot. As shown in Fig. 14, Wif1 is not only expressed by ASZ-Wif1 but also secreted. 


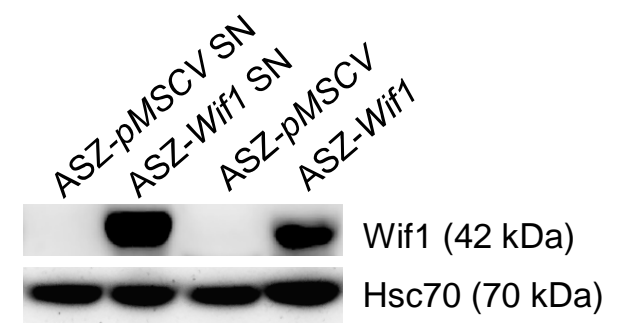

Fig. 14: Western blot detecting Wif1 in supernatant and cellular extracts of ASZ-pMSCV and ASZ-Wif1 Antibody detecting the housekeeper protein $\mathrm{Hsc70}$ was used as loading control. Abbreviation: SN: concentrated supernatant.

\section{IV.3 Functional analysis of Wif1 in vitro}

Wif1 is a well-known inhibitor of Wnt signaling due to sequestration of Wnt ligands in the extra cellular space. Orthologs of human WIF1 have also been shown to interact with $\mathrm{Hh}$ thereby promoting its activity. In addition, the Wnt and $\mathrm{Hh}$ signaling pathways can influence each other at several interfaces. To investigate whether Wif1 can modulate the activity of the latter signaling cascades, I employed functional assays and analyzed target gene expression of the respective pathways. To analyze the function of Wif1 in vitro on Wnt and Hh signaling, I analysed the expression of Axin2 and Gli1, respectively. I also tried to establish the TOP/FOP reporter assay, which is useful to analyze the activity of the Wnt signaling pathway after its induction with Wnt ligands.

\section{IV.3.1 TOP/FOP reporter assay for assessment of Wif1 activity}

In order to analyze the inhibitory capability of Wif1 on Wnt ligands and thus on Wnt signaling activity, the TOP/FOP reporter assay was used as described in III.14.4.9.

As mentioned above ASZ001 are hard to transfect using lipid based transfection systems. However, nucleofection improved the plasmid transfer in ASZ001 resulting in 50 \% eGFP positive cells when using the reporter 
plasmid pGFPmax (data not shown). Unfortunately, this method significantly reduced ASZ001 cell viability (data not shown).

Nevertheless, ASZ001 were transfected with the TOP plasmid. Cotransfection with $\mathrm{pCl}$-neo- $\beta$-cateninS33Y encoding for a mutationally, constitutively activated $\beta$-catenin (S33Y) served as positive control. TOP transfected cells were incubated with Wnt3a conditioned medium derived from stably transfected $L$ cells (Wnt3a $C M$ ). Conditioned medium from untransfected $L$ cells ( $L C M$ ) served as control medium. As negative control, ASZ001 were transfected with the FOP and S33Y plasmids (data not shown).

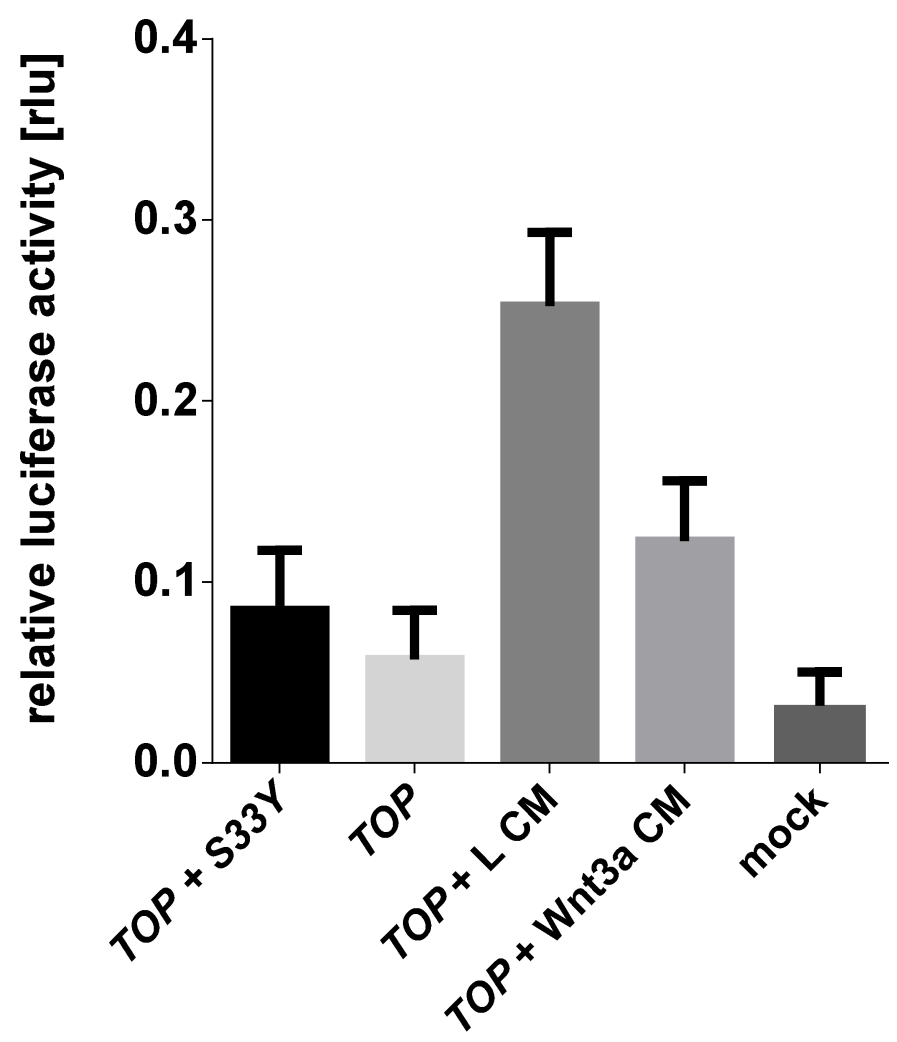

Fig. 15: TOP/FOP reporter assay of Wnt signaling in ASZ001 cells incubated with Wnt3a

As positive control ASZ001 cells were co-nucleofected with SuperTOPFlash (TOP) and pCl-neo- $\beta$-cateninS33Y (S33Y). As negative control ASZ001 were co-nucleofected with SuperFOPFlash (FOP) and S33Y. ASZ001 cells only nucleofected with TOP or TOP transfected cells incubated with conditioned medium of untransfected L cells ( $\mathrm{L}$ $\mathrm{CM}$ ) were used as further controls. In addition, TOP nucleofected cells were incubated with Wnt3a conditioned medium from $L$ cells stably expressing and secreting Wnt3a in order to activate canonical Wnt signaling driving luciferase expression (Wnt3a CM). Firefly luciferase activity was normalized to Renilla luciferase activity, which is under CMV promoter control. All data are represented as mean + SEM. Statistical significance was tested using Mann-Whitney $U$ test. rlu: relative light units. 
However, neither the positive control TOP + S33Y nor Wnt3a CM did induce luciferase activity in this assay. Therefore, we decided to change the experimental setup and employed the easy-to-transfect cell line HEK-293 for further experiments. For activation of Wnt signaling Wnt3a CM was used. The 75-fold concentrated supernatant of ASZ-Wif1 cells (Wif1 conc. SN, see Fig. 16) was employed and 75-fold concentrated supernatant of ASZ-pMSCV (pMSCV conc. SN) served as control. As additional controls of successful Wht signaling inhibition, I used recombinant dickkopf1 (rDkk1) at a concentration of $750 \mathrm{ng} / \mathrm{ml}$ and recombinant Wif1 (rWif1) protein at a concentration of $30 \mu \mathrm{g} / \mathrm{ml}$. In HEK-293 cells, all negative controls (i.e. TOP, $F O P$ or $F O P+S 33 Y$ ) did not induce luciferase activity. In contrast, transfection with $T O P+S 33 Y$ highly induced luciferase activity. Cells that were transfected with $T O P$ in combination with a $24 \mathrm{~h}$ incubation with a 1:1 mixture of Wnt3a CM and pMSCV conc. SN moderately induced luciferase activity (24 $\mathrm{h}$ incubation with Wnt3a CM alone resulted in an equal induction of luciferase activity, data not shown). This induction was significantly reduced by Wif1 conc. SN comparable with $30 \mu \mathrm{g} / \mathrm{ml}$ rWif1 and to a lesser extent compared with $750 \mathrm{ng} / \mathrm{ml}$ rDkk1. 


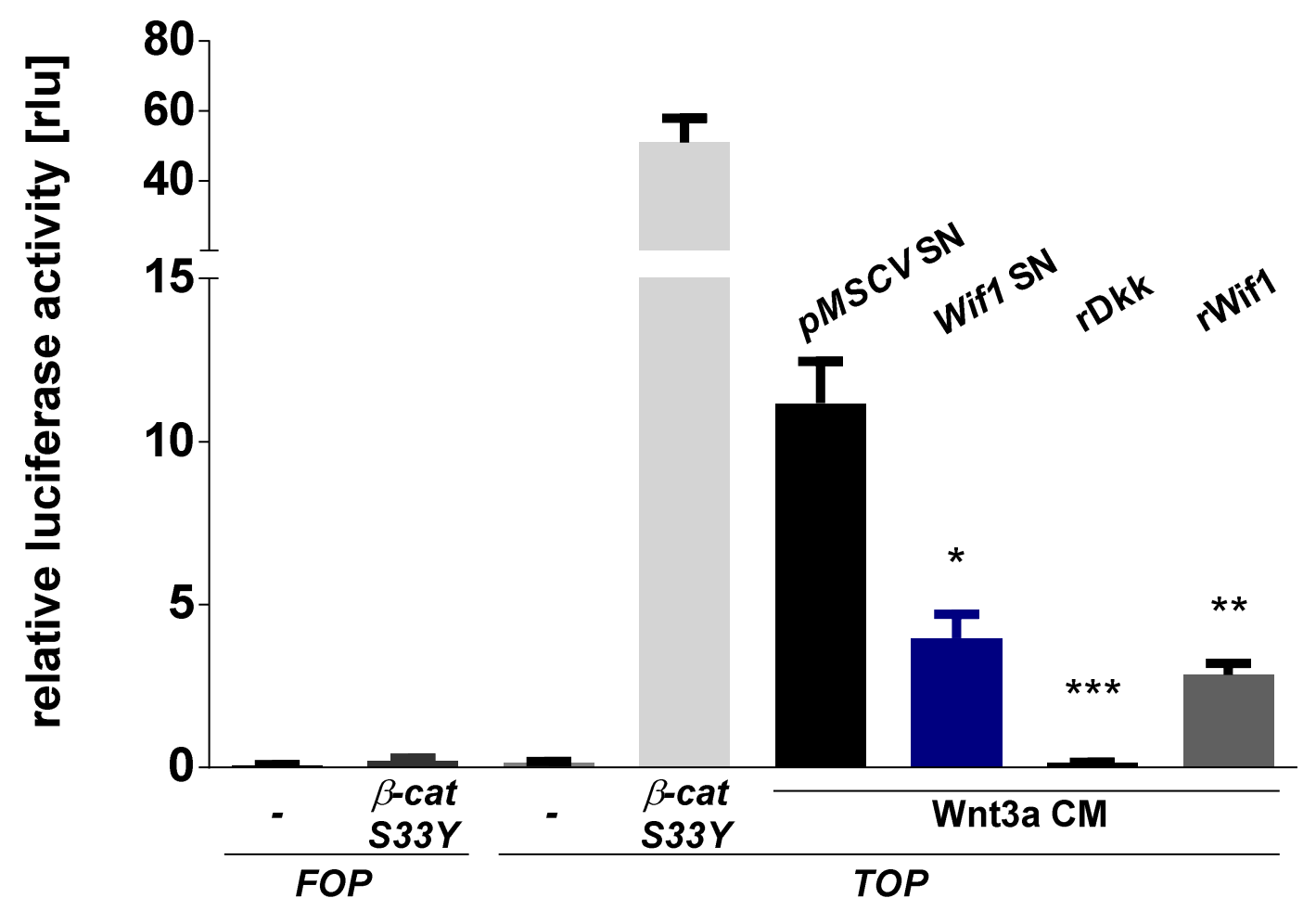

Fig. 16: TOP/FOP reporter assay in HEK-293 cells incubated with Wnt3a and Wif1-concentrated supernatant As positive control HEK-293 cells were co-nucleofected with SuperTOPFlash (TOP) and pCl-neo- $\beta$-cateninS33Y ( $\beta$-cat S33Y). As negative control HEK-293 were co-nucleofected with SuperFOPFlash (FOP) and S33Y. HEK-293 cells only nucleofected with TOP or TOP transfected cells incubated with conditioned medium of untransfected $\mathrm{L}$ cells ( $\mathrm{LCM}$ ) were used as further controls. In addition, TOP nucleofected cells were incubated with Wnt3a conditioned medium from $L$ cells stably expressing and secreting Wnt3a in order to activate canonical Wnt signaling driving luciferase expression (Wnt3a $\mathrm{CM}$ ). Moreover, cells were incubated with concentrated supernatant from ASZ-pMSCV (pMSCV SN) and from ASZ-Wif1 (Wif1 SN), respectively. Firefly luciferase activity was normalized to Renilla luciferase activity, which is under $\mathrm{CMV}$ promoter control. As a control for efficient Wnt inhibition recombinant dickkopf1 protein (rDkk1) $(750 \mathrm{ng} / \mathrm{ml})$ and recombinant Wif1 protein ( $\mathrm{rWif1})(30 \mu \mathrm{g} / \mathrm{ml})$ were used. Firefly luciferase activity was normalized to Renilla luciferase activity. rlu: relative light units. All data are represented as mean + SEM. Statistical significance was tested using Mann-Whitney $U$ test. ${ }^{*} p<0.05,{ }^{* *} p<0.005,{ }^{* * *} p<0.001$.

Together, these data demonstrate that ASZ-Wif1 cells express and secrete functionally active Wif1.

\section{IV.3.2 Analysis of $\mathrm{Hh}$ and Wnt pathway activity and $\mathrm{Ca}^{2+}$ measurement}

Axin2 and Gli1 are considered as the most general and common target genes of active Wnt and $\mathrm{Hh}$ signaling, respectively. Analysis of ASZ-pMSCV 
and ASZ-Wif1 by means of qRT-PCRs showed no significant differences in expression levels of both genes (see Fig. 17).

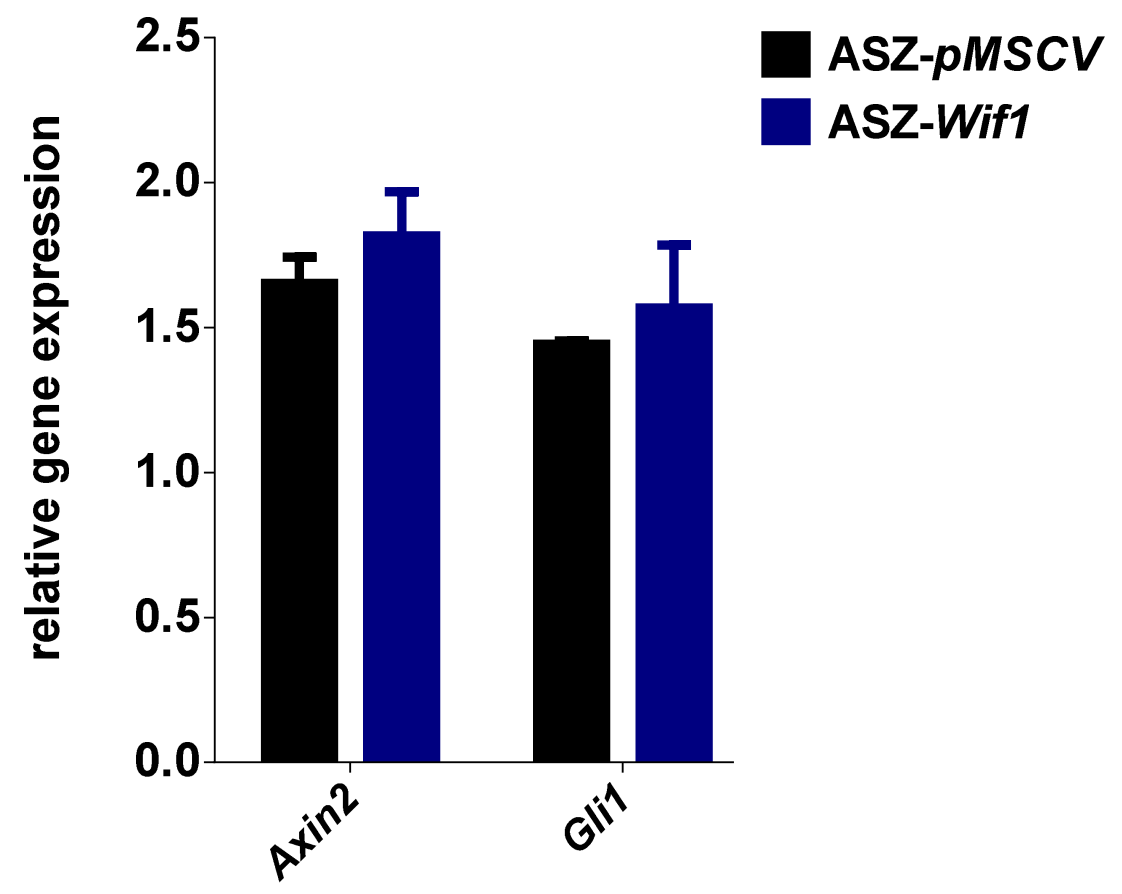

Fig. 17: qRT-PCR of Axin2 and Gli1 in ASZ-pMSCV and -Wif1

Relative Axin2 and Gli1 expression in ASZ-pMSCV and -Wif1. Values were normalized to Hprt expression. All data are represented as mean + SEM. Statistical significance was tested using Mann-Whitney $U$ test.

However, as both cell lines do not feature a priori activated Wnt signaling, it cannot be ruled out that Wif1 might have affected Axin2 expression in an activated status of Wnt signaling. Unfortunately, Wnt3a transfected $L$ cells do not secrete Wnt3a when cultured in $154 \mathrm{CF}$ medium that is used to propagate ASZ001. Vice versa, ASZ001 differentiate in DMEM based medium due to high $\mathrm{Ca}^{2+}$ concentrations, biasing Wnt target gene expression analysis.

Next, the cytoplasmic $\mathrm{Ca}^{2+}$ concentrations as an indicator for active $\mathrm{Wnt} / \mathrm{Ca}^{2+}$ signaling were examined. In addition the $\mathrm{Ca}^{2+}$ influx from $\mathrm{ER}$ into the cytoplasm was analyzed by $\mathrm{Ca}^{2+}$-influx assay (see Fig. 18). 

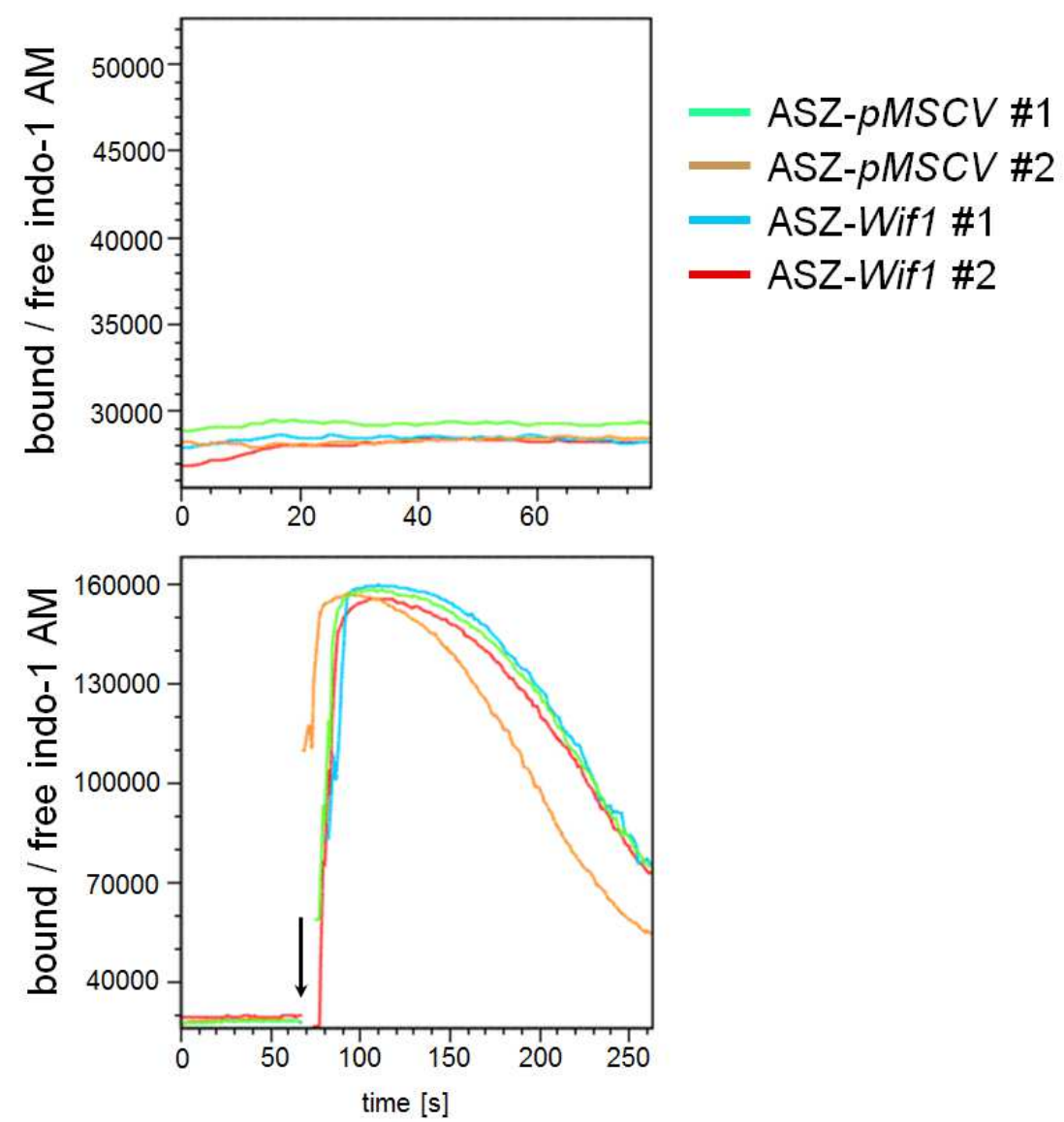

Fig. 18: $\mathrm{Ca}^{2+}$-influx assay of ASZ-pMSCV and -Wif1

Ratio of bound and free indo-1 AM was measured for $60 \mathrm{~s}$ to determine the basal $\mathrm{Ca}^{2+}$ levels (top). After $60 \mathrm{~s}$ $1 \mu \mathrm{g} / \mathrm{ml}$ ionomycin was added (arrow) to establish a maximum Calcium flux ratio for each cell line (bottom).

However, neither the basal cytoplasmic $\mathrm{Ca}^{2+}$ concentrations nor the maximum $\mathrm{Ca}^{2+}$ flux ratio was altered in ASZ-Wif1 compared to ASZ-pMSCV.

\section{IV.4 Effect of Wif1 overexpression on various cellular processes of ASZ001 in vitro}

Wif1 has been implicated in various cellular processes including the regulation of cell viability, apoptosis, migration, epithelial-to-mesenchymal transition (EMT), differentiation, cell cycle progression and proliferation. The impact of Wif1 on these processes was analyzed in vitro in ASZ-Wif1 and ASZ-pMSCV control cells. 


\section{IV.4.1 Effect of Wif1 on cell viability in vitro}

Cell viability was analyzed by WST-1 assay. 5.000 cells/well were seeded in 96-well plates and cultured for $48 \mathrm{~h}$ in total. Incubation for the last $24 \mathrm{~h}$ with $200 \mathrm{mM} \mathrm{H}_{2} \mathrm{O}_{2}$ served as a control for reduced cell viability. WST-1 assay was performed 3 times in triplicates. As expected, incubation with $200 \mathrm{mM} \mathrm{H}_{2} \mathrm{O}_{2}$ significantly reduced cell viability compared to untreated cells. However, no significant difference between the cell lines were detected, neither after incubation with $\mathrm{H}_{2} \mathrm{O}_{2}$ nor between untreated ASZ-pMSCV and ASZ-Wif1 cells (see Fig. 19; shown is a representative experiment).

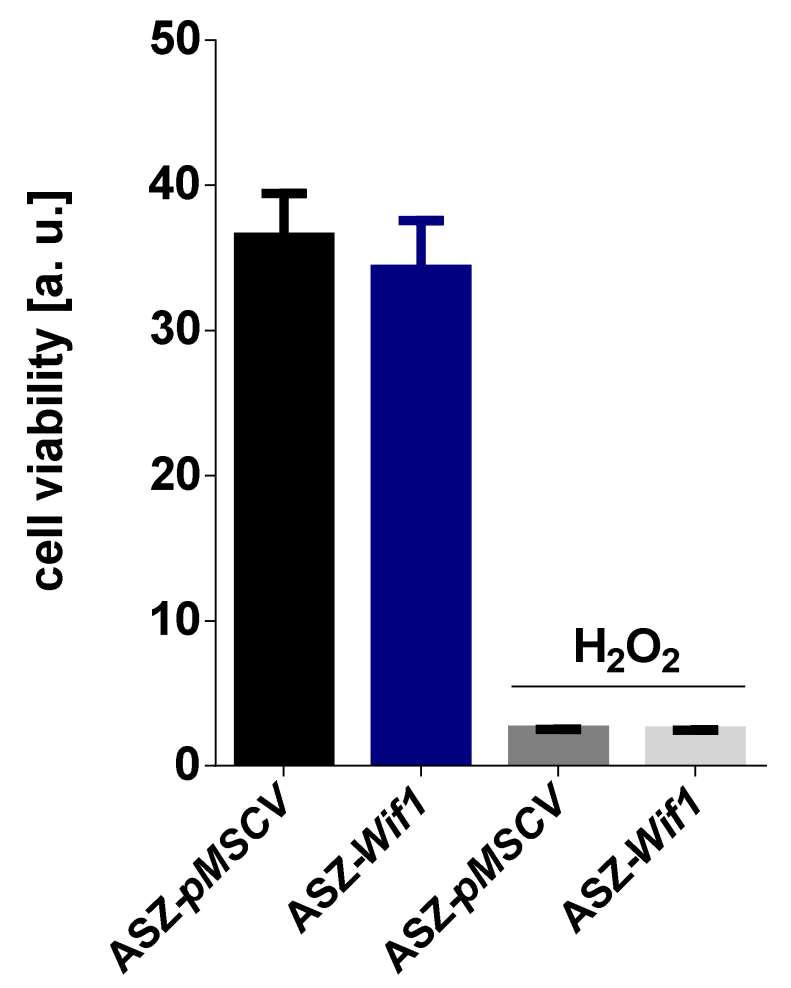

Fig. 19: WST-1 assay of ASZ-pMSCV and -Wif1

ASZ-pMSCV and -Wif1 cells were cultured untreated in 154 CF supplemented with $2 \%$ chelexed FCS and $1 \% \mathrm{P} / \mathrm{S}$ for $48 \mathrm{~h}$ in total or treated for the last $24 \mathrm{~h}$ with $200 \mathrm{mM} \mathrm{H}_{2} \mathrm{O}_{2}\left(\mathrm{H}_{2} \mathrm{O}_{2}\right)$. All data are represented as mean + SEM. [a. u.]: arbitrary units. Statistical significance was tested using Mann-Whitney $U$ test.

\section{IV.4.2 Effect of Wif1 on apoptosis in vitro}

In order to investigate the effect of Wif1 on apoptosis in vitro we used an Annexin V assay as described in III.14.4.13. Shown is a representative experiment performed in duplicates. As a positive control $1 \mu \mathrm{M}$ of the protein kinase inhibitor staurosporine (stauro) was applied for $24 \mathrm{~h}$. Staurosporine 
significantly induced apoptosis in both cell lines. However, no significant difference between ASZ-pMSCV and ASZ-Wif1 cell lines was detected. The difference was also not significant when the values of untreated ASZ-pMSCV and ASZ-Wif1 were compared (see Fig. 20).

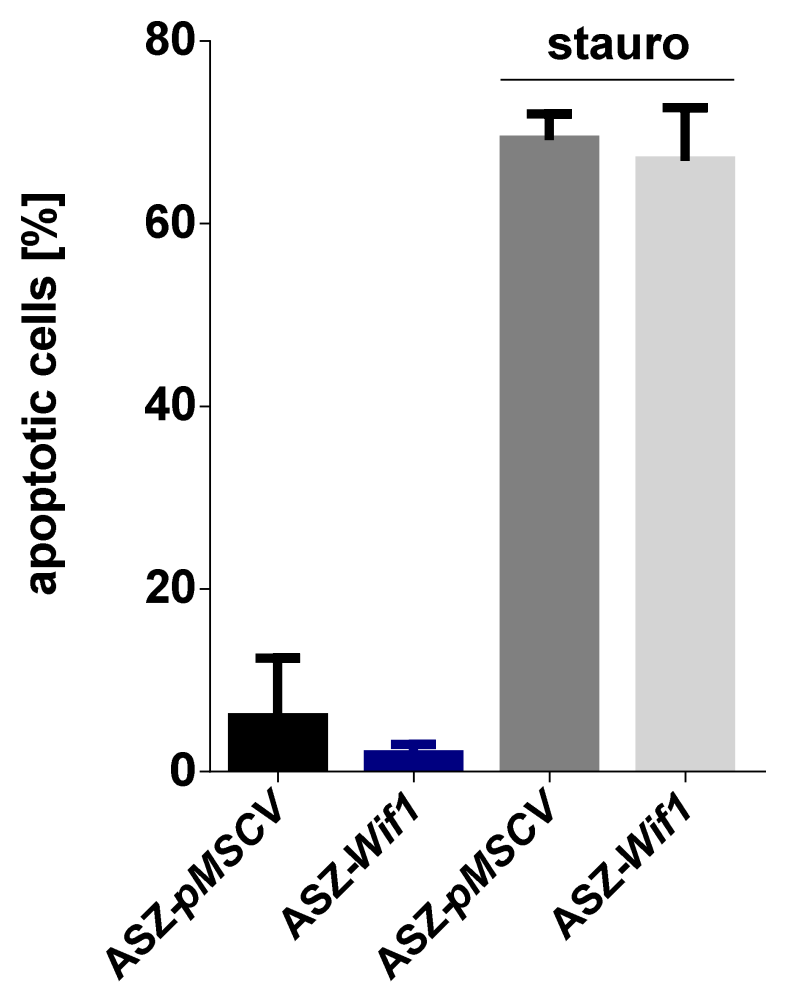

Fig. 20: Annexin V assay of uninduced and staurosporine induced ASZ-pMSCV and -Wif1

Annexin $\mathrm{V}$ negative, propidium iodide (PI) positive cells were considered as necrotic (not shown). Annexin $\mathrm{V}$ negative, propidium iodide ( $\mathrm{PI}$ ) negative cells were considered as living cells (not shown). Annexin $\mathrm{V}$ positive cells were considered as apoptotic. Cells were treated either with DMSO or $1 \mu \mathrm{M}$ staurosporine (stauro) for $24 \mathrm{~h}$. Respective proportion of cells that are apoptotic is given in percent. All data are represented as mean + SEM. Statistical significance was tested using Mann-Whitney $U$ test.

\section{IV.4.3 Effect of Wif1 on cell cycle regulation and proliferation in vitro}

To analyze the effect of Wif1 on cell cycle regulation a PI staining was performed. Compared to ASZ-pMSCV the cell line ASZ-Wif1 showed a tendency to accumulate in the G1 phase of the cell cycle. This resulted in a reduced proportion of cells in the G2/M phase (see Fig. 21), which, however, was statistically not significant. 


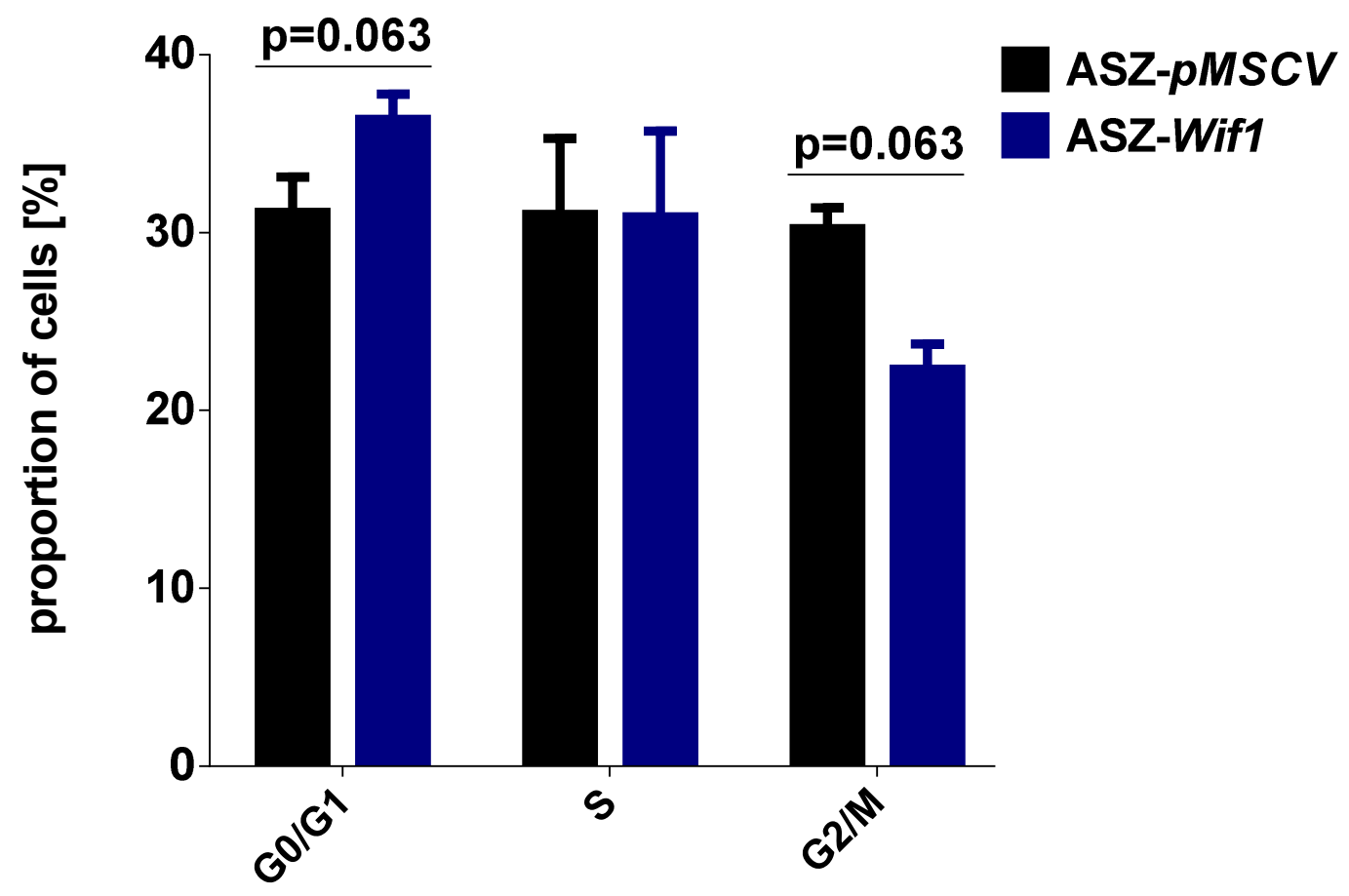

Fig. 21: Distribution of ASZ-pMSCV and -Wif1 in respective phases of cell cycle

All values represent the mean + SEM of 2 independent experiments performed in duplicates in G0/G1, S and G2/M phase of cell cycle. Statistical significance was tested using Wilcoxon test.

In order to investigate whether the accumulation of the cells in $\mathrm{G} 1$ was associated with changes in DNA replication, a BrdU assay was performed. The cells were cultured for $72 \mathrm{~h}$ and labelled with BrdU for the last $24 \mathrm{~h}$. The analysis was conducted 3 times in sextuplicates. Shown is a representative experiment (see Fig. 22). However, there was no significant difference in cellular proliferation when comparing the values for ASZ-pMSCV and ASZ-Wif1. 


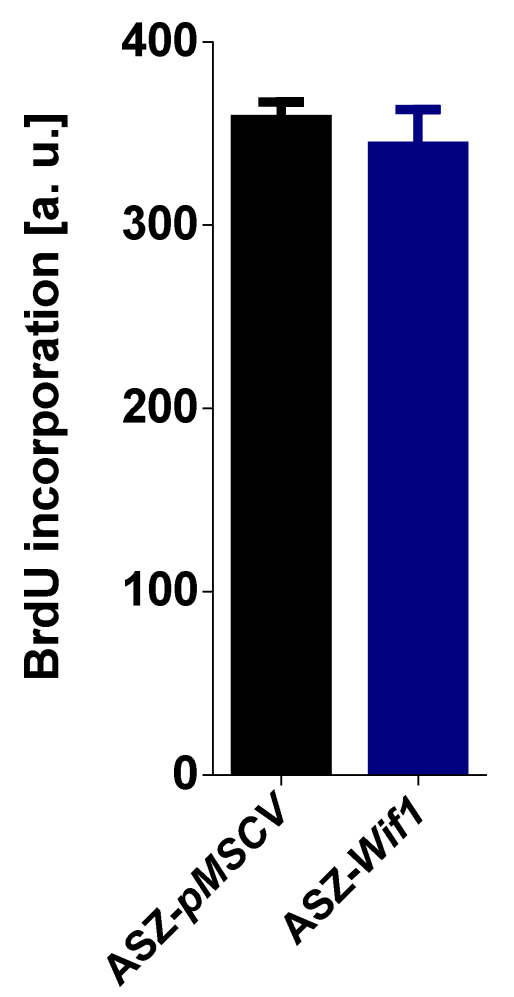

Fig. 22: BrdU assay of ASZ-pMSCV and -Wif1

ASZ-pMSCV and -Wif1 cells were cultured untreated in regular culture medium for $72 \mathrm{~h}$. BrdU was added for the last $24 \mathrm{~h}$. All data are represented as mean + SEM. Statistical significance was tested using unpaired t test with Welch`s correction.

Moreover, ASZ-pMSCV and ASZ-Wif1 cells were cocultivated with Wnt3A L and $\mathrm{L}$ cells, with Wnt5a-NIH/3T3 and $\mathrm{NIH} / 3 \mathrm{~T} 3$ cells, with B9 (murine fibroblasts) and murine bone marrow derived macrophages (BMDMs). This allowed for examination of potential effects of Wif1 on cellular proliferation in response to cues derived from adjacent fibroblasts or macrophages. For this purpose, ASZ-pMSCV and ASZ-Wif1 were seeded in a 24-well plate. On the same day, the cell lines used for coculture were seeded in 24-well plate inserts and the inserts were placed in the 24-well plate. On each day of 5 consecutive days the cell numbers were counted using the CeligoS device and corresponding software, taking 24 serial images of each well (see Fig. 23). 

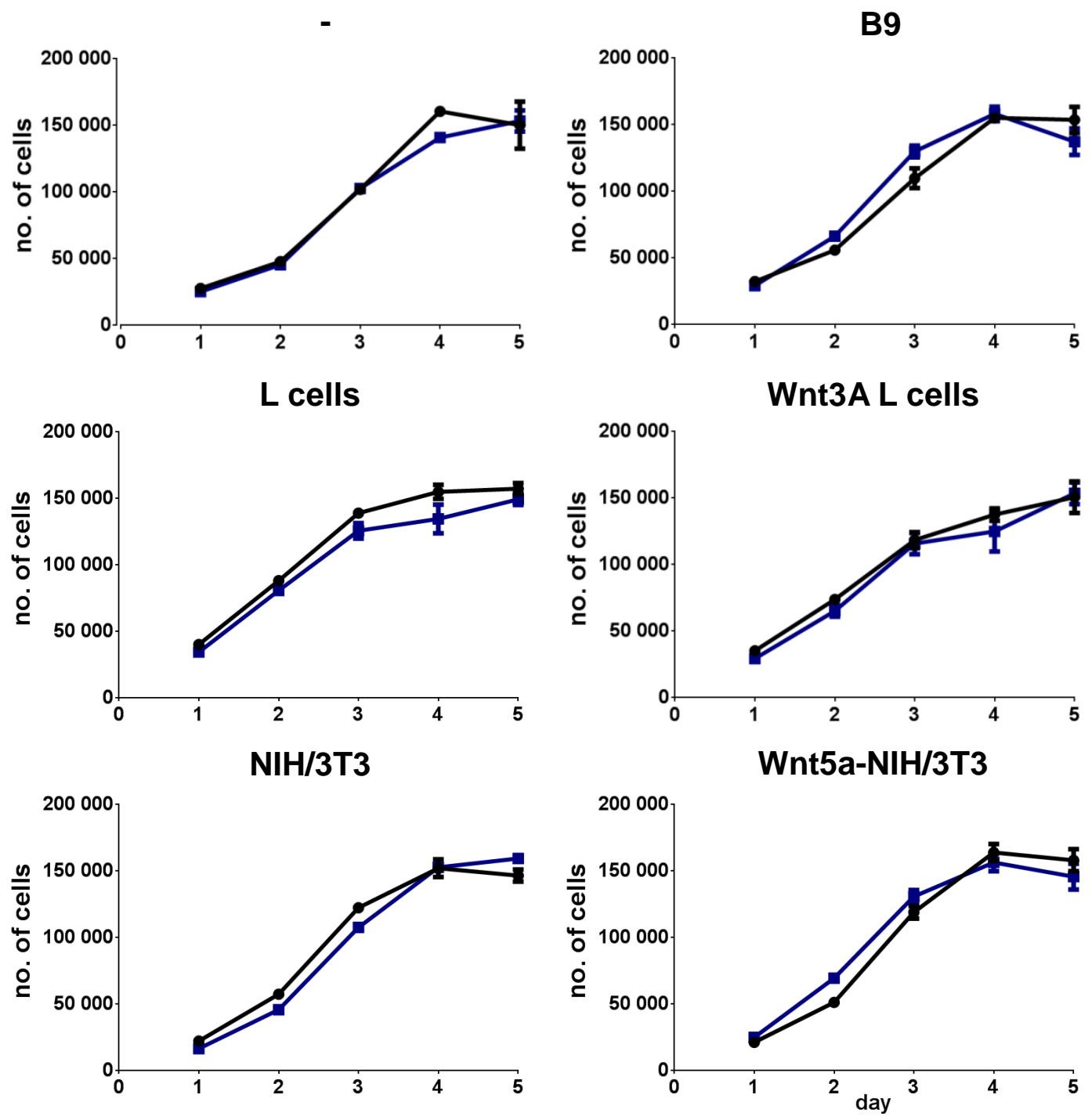

BMDMs

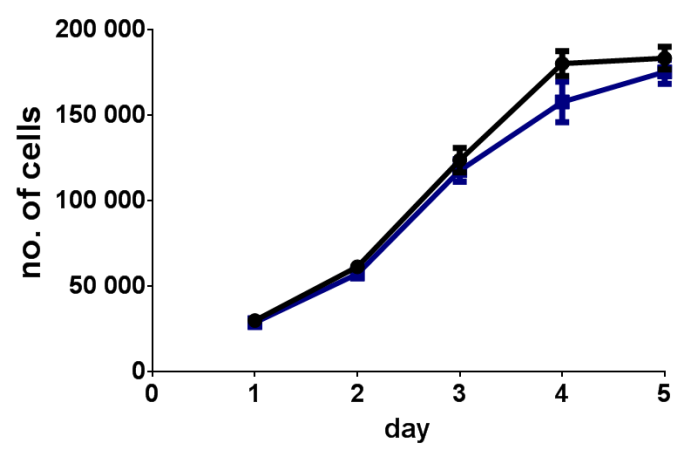

ASZ-pMSCV

ASZ-Wif1

Fig. 23: Cell proliferation assay of ASZ-pMSCV and ASZ-Wif1 cocultured with B9, L cells, Wnt3A L cells, NIH/3T3, Wnt5a-NIH/3T3 or BMDMs

ASZ-pMSCV, ASZ-Wif1 and cocultured cells were seeded on day 0 in 24-well plates or 24-well plates inserts, respectively. Cells were counted each day of 5 consecutive days. All data are represented as mean \pm SEM.

ASZ-pMSCV and ASZ-Wif1 cells showed an exponential growth and reached the plateau phase between day 4 and day 5 . This was seen in all settings. 
None of the cocultured cells elevated or decreased the proliferation of ASZ-Wif1 when compared to ASZ-pMSCV.

In summary, these data suggest that Wif1 does not alter the proliferative capacity of ASZ001 in vitro.

\section{IV.4.4 Effect of Wif1 on migration in vitro}

As Wif1 has also been shown to inhibit migration of several cancer cell lines (Yee et al. 2010; Vassallo et al. 2015), the migratory capacity of ASZ-pMSCV and ASZ-Wif1 was investigated in a transwell migration assay (see Fig. 24). However, the migratory capacity of ASZ-Wif1 cells did not show any significant difference compared to ASZ-pMSCV cells.

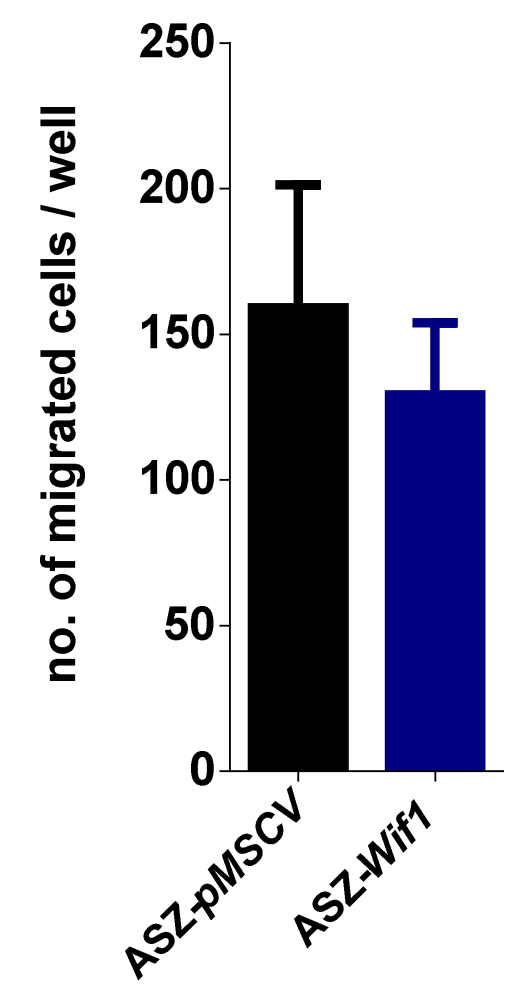

Fig. 24: Transwell migration assay of ASZ-pMSCV and -Wif1

Cells were seeded in 24-well plate inserts. $16 \mathrm{~h}$ later cells that had migrated through $8.0 \mu \mathrm{m}$ pores were counted. All data are represented as mean + SEM. Statistical significance was tested using Wilcoxon test.

\section{IV.4.5 Effect of Wif1 on differentiation in vitro}

The cellular morphology of ASZ-Wif1 and ASZ-pMSCV cells was examined microscopically. No obvious alterations in cell morphology were observed. Next, the expression of the differentiation markers Krt1 and Krt10 were 
analyzed by means of qRT-PCR. Krt1 was not expressed and Krt10 expression was not changed significantly in ASZ-Wif1 compared to ASZ-pMSCV.

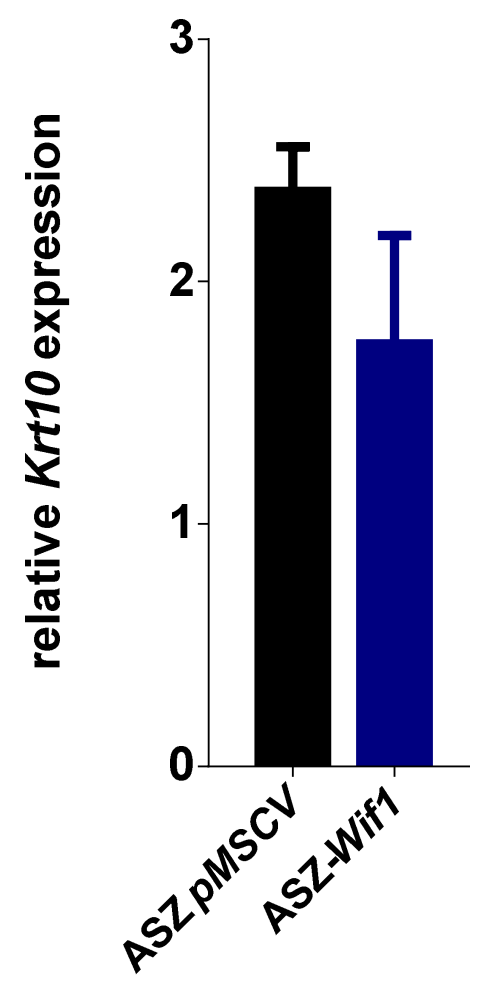

Fig. 25: qRT-PCR of Krt10 in ASZ-pMSCV and -Wif1

Relative Krt10 expression in ASZ-pMSCV and -Wif1. Values were normalized to Hprt expression. All data are represented as mean + SEM. Statistical significance was tested using Mann-Whitney $U$ test.

\section{IV.5 Effect of Wif1 in vivo}

In order to investigate the influence of Wif1 on BCC growth in mice, $1 \cdot 10^{6}$ cells of both cell lines suspended in matrigel were subcutaneously transplanted in the flanks of nude mice. In order to exclude individual mouse effects, each mouse was injected with $200 \mu \mathrm{l}$ of ASZ-pMSCV and ASZ-Wif1 cell suspension in matrigel on the left and right flank, respectively. When reaching a size of $5 \mathrm{~mm}$, tumor size was measured every second day using a caliper. Measurement was conducted until general health conditions or tumor size required the termination of the experiment. The age of the 17 mice upon transplantation was 10 weeks. 
As demonstrated in Fig. 26, the minimum size of the tumors (i.e. $5 \mathrm{~mm}$ ) was generally reached at day 17 after transplantation. Then the tumors showed an approximately exponential growth until day 33 after injection. Tumors that arose from transplanted ASZ-pMSCV cells grew significantly faster compared to tumors that originated from ASZ-Wif1 cells (see Fig. 26). Indeed, the size of ASZ-pMSCV-derived tumors was significantly larger at any time point during measurement.

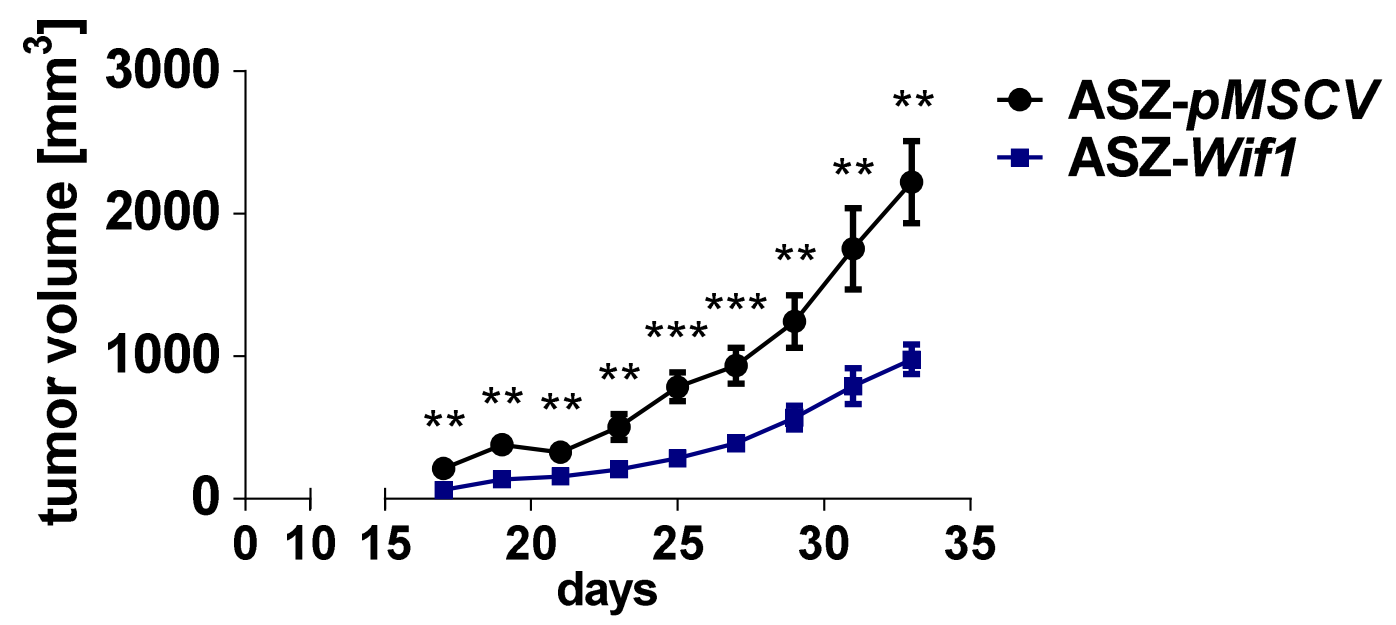

Fig. 26: Growth curve of ASZ-pMSCV and ASZ-Wif1 allografts in nude mice

$1 \cdot 10^{6}$ cells in a $200 \mu \mathrm{l}$ cell suspensions in matrigel of either ASZ-pMSCV or ASZ-Wif1 were injected in the left or right flank, respectively, in 17 nude mice. After the tumors reached a minimum diameter of $5 \mathrm{~mm}$ the tumor size were measured every other day. All data are represented as mean \pm SEM. Statistical significance was tested using Wilcoxon test. ${ }^{* *} p<0.005,{ }^{* * *} p<0.001$.

The significant difference in tumor sizes was also reflected by a significantly reduced tumor weight of tumors originating from transplanted ASZ-Wif1 cells. (see Fig. 27). 


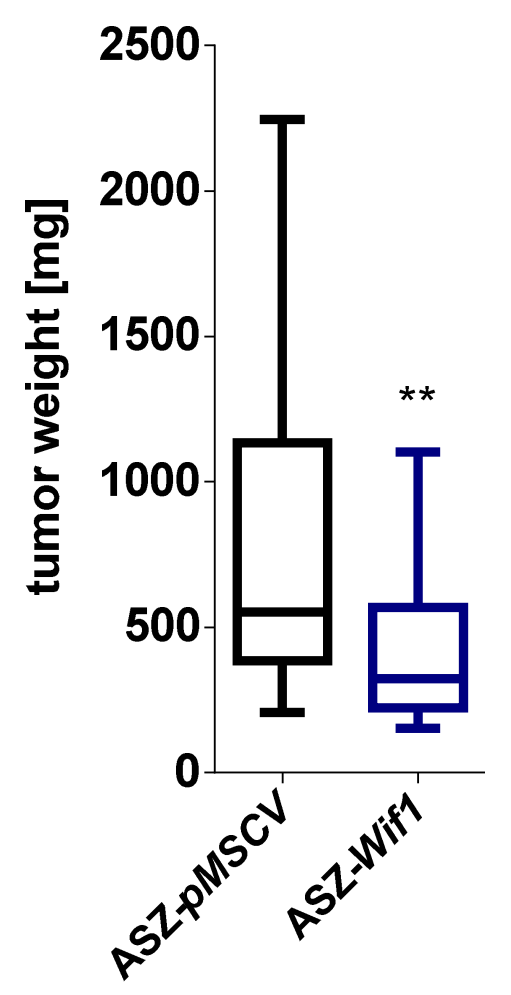

Fig. 27: Tumor weight of ASZ-pMSCV and ASZ-Wif1 allografts after dissection

$1 \cdot 10^{6}$ cells in a $200 \mu \mathrm{l}$ cell suspensions in matrigel of either ASZ-pMSCV or ASZ-Wif1 were injected in the left or right flank, respectively, in 17 nude mice. After the termination of the experiment, tumors were removed and weighed. All data are represented as a box-whisker plot: median (horizontal line), 25-75 percentile (box), 5-95 percentile (whiskers). Statistical significance was tested using Wilcoxon test. ${ }^{* *} p<0.005$.

Similar results were obtained when $1 \cdot 10^{6}$ cells originating from 3 different single cell clones of both cell lines (see section IV.2.2 and Fig. 13) were transplanted (data not shown).

In Fig. 28 representative images of H\&E stainings of ASZ-pMSCV and ASZ-Wif1 derived allografts are depicted. Both groups of allografts show growth of tumor nodules surrounded by stroma, spots of differentiation in form of keratinization (arrows) and areas of necrosis (asterisks) (see Fig. 28). 


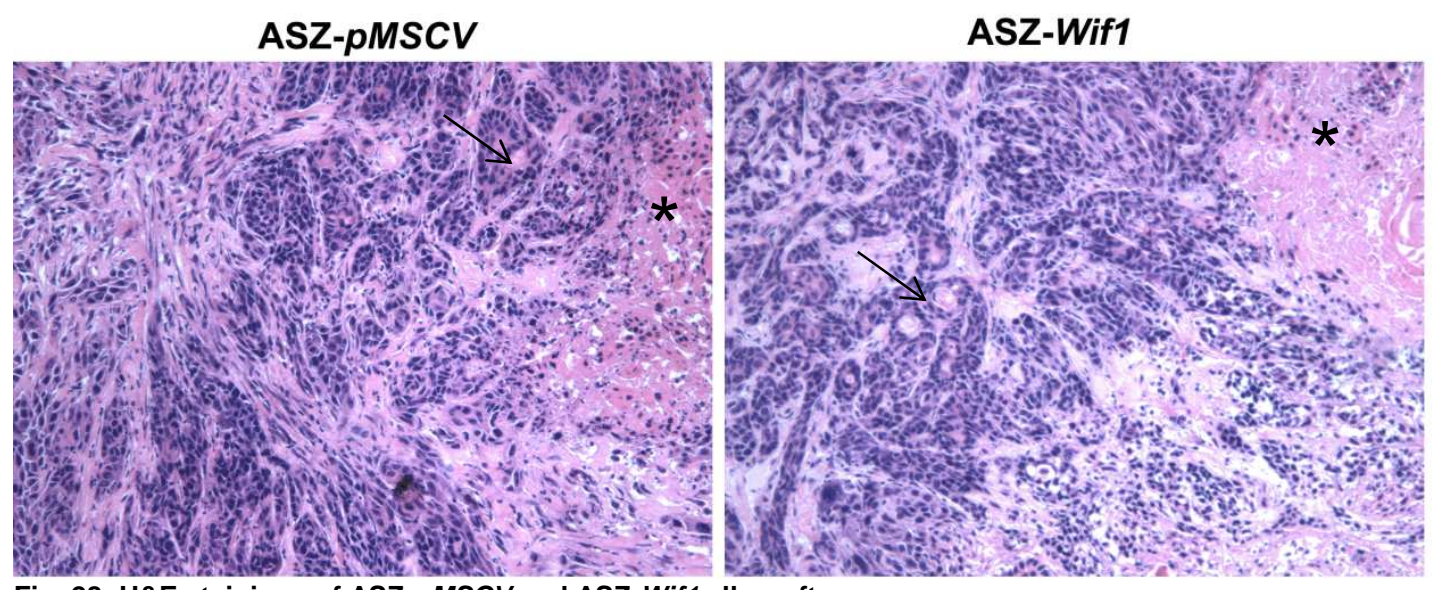

Fig. 28: H\&E stainings of ASZ-pMSCV and ASZ-Wif1 allografts

Arrows indicate spots of keratinization. Asterisks indicate necrotic areas.

In order to rule out that the difference in tumor size between ASZ-pMSCV and ASZ-Wif1 derived tumors was not simply caused by swelling of ASZ-pMSCV allografts due to increased keratinization and necrosis, the areas which were clearly necrotic or keratinous were subtracted from the total tumor area. This was done on serial images using the software cellSens. Fig. 29 shows two examples of the total tumor (overlay of serial images). The red lines indicate the borders of vital tumor tissue and necrotic/ keratinous areas.
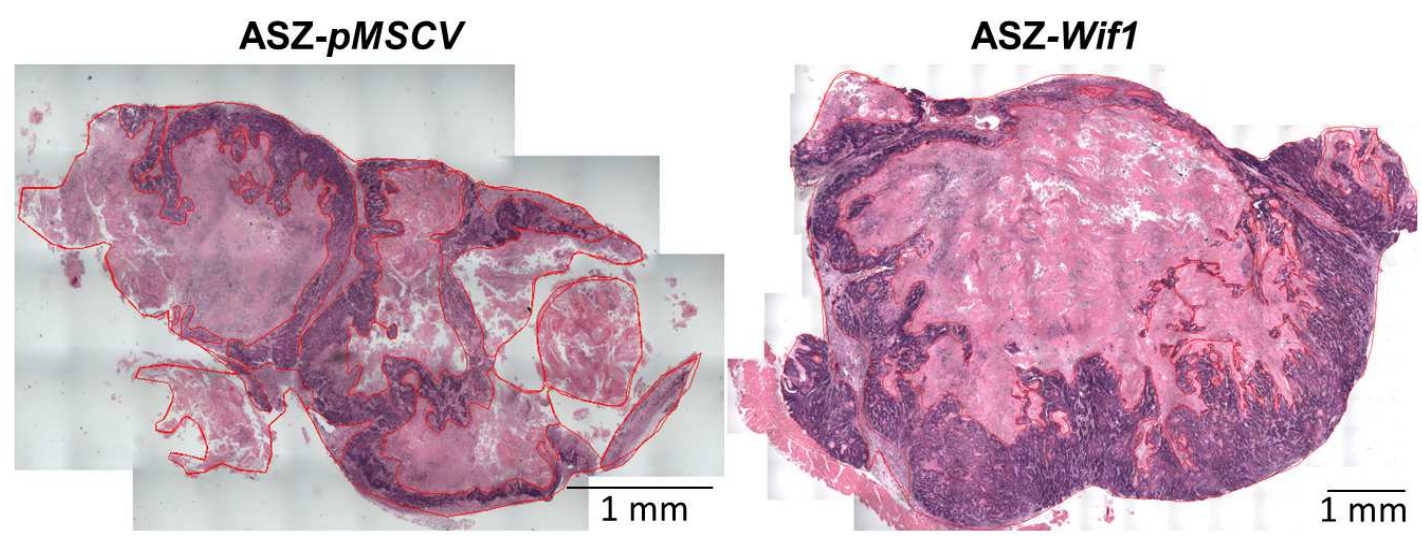

Fig. 29: Total sections of ASZ-pMSCV and ASZ-Wif1 allografts

Necrotic and keratinous areas were encircled and separated from the total tumor areas by red lines. Image acquisition, image overlay and area demarcation were performed using cellSens software.

The proportion of necrotic and keratinous areas in ASZ-pMSCV allografts was moderately decreased compared to ASZ-Wif1 allografts. However, this difference was statistically not significant (see Fig. 30). 


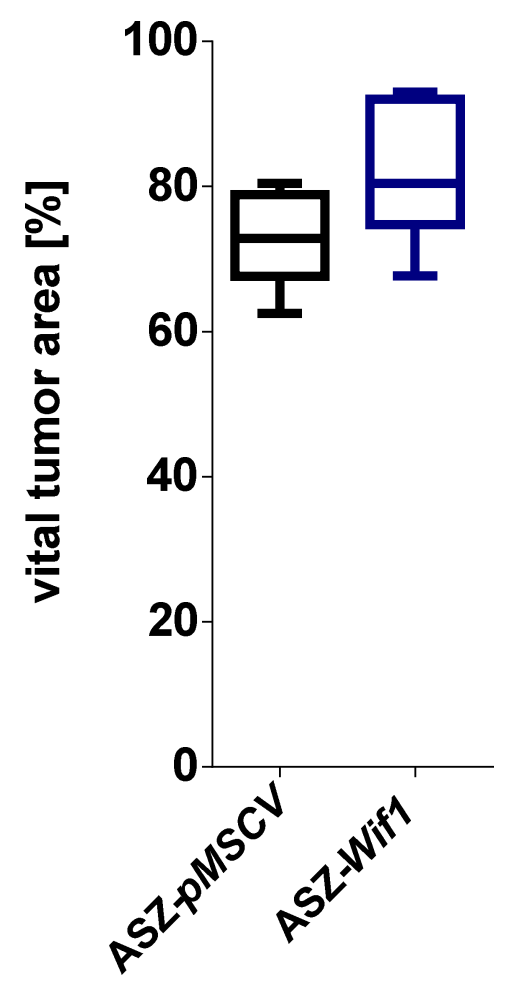

Fig. 30: Vital tumor area of ASZ-pMSCV and ASZ-Wif1 allografts

Necrotic and keratinous areas were measured and subtracted from total tumor area of 7 different ASZ-pMSCV and ASZ-Wif1 derived tumors. Image acquisition and area calculation was performed using cellSens software. All data are represented as a box-whisker plot: median (horizontal line), 25-75 percentile (box), 5-95 percentile (whiskers). Statistical significance was tested using Wilcoxon test.

\section{IV.5.1 Effect of Wif1 on Hh, Wnt and Akt signaling pathways in vivo}

In order to analyze the effect of Wif1 overexpression on Wnt and $\mathrm{Hh}$ signaling, target gene expression analysis of both pathways was performed. Ten tumor samples from each group (i.e. ASZ-pMSCV and ASZ-Wif1 derived tumors) were analyzed.

When the Hh target Gli1 was measured, the tumors did not show different levels of Gli1 expression indicating comparable $\mathrm{Hh}$ signaling activity in ASZ-pMSCV and ASZ-Wif1 allografts (see Fig. 31). 


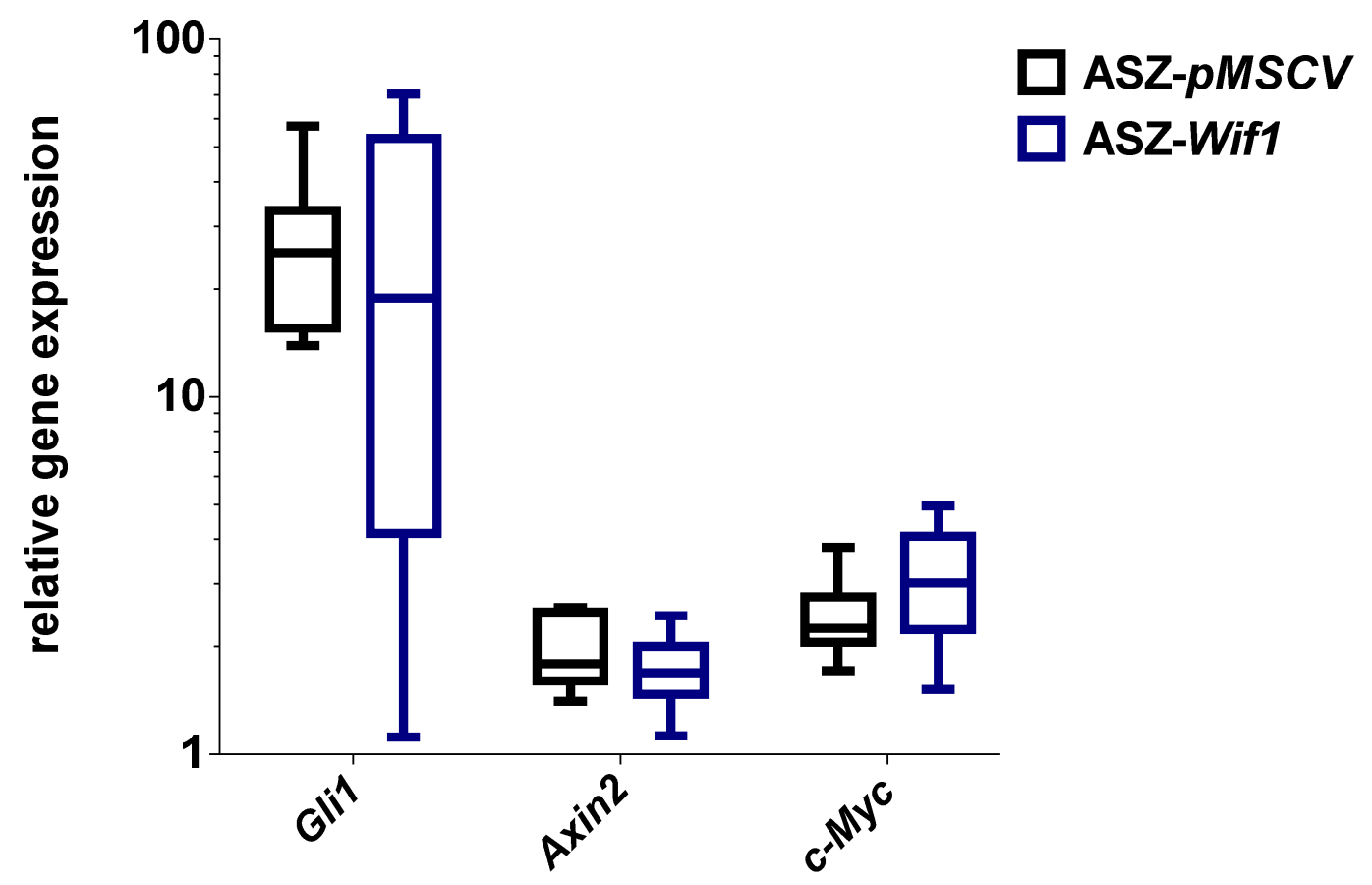

Fig. 31: qRT-PCR of Gli1, Axin2 and c-Myc expression in ASZ-pMSCV and ASZ-Wif1 allografts

Each bar summarizes data of 10 individual ASZ-pMSCV or ASZ-Wif1 derived tumors. Values were normalized to Hprt expression. Statistical significance was tested using Wilcoxon test. All data are represented as a box-whisker plot: median (horizontal line), 25-75 percentile (box), 5-95 percentile (whiskers). Statistical significance was tested using Wilcoxon test.

Similarly, Wif1 overexpression did not influence the expression of $c-M y c$ and Axin2. This indicates that canonical Wnt signaling was not affected (see Fig. 31). Moreover, translocation of $\beta$-catenin into the nucleus, which is a hallmark of active canonical Wnt signaling, was investigated. For this purpose, antibody staining of $\beta$-catenin was performed.

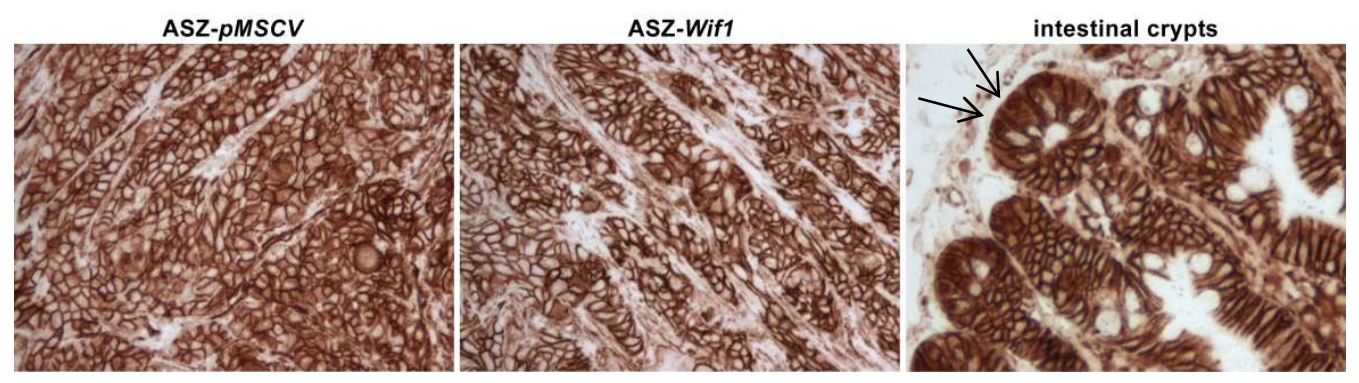

Fig. 32: Antibody staining of $\beta$-catenin in ASZ-pMSCV and ASZ-Wif1 allografts

Anti-ß-catenin antibody staining of paraffin sections derived from ASZ-pMSCV and ASZ-Wif1 allografts. Images were taken at 100 -fold magnification. Intestinal crypts served as positive control for nuclear staining of $\beta$-catenin (arrows). Staining reaction was performed using DAB+. 
As shown in Fig 32, Wif1 also did not affect $\beta$-catenin translocation. Together, absent nuclear $\beta$-catenin staining and unchanged target gene expressions in ASZ-pMSCV and ASZ-Wif1 derived allografts indicate that Wif1 does not affect canonical Wnt signaling.

Next, the phosporylation status of JNK and CaMKII was examined, which are targets of the $\beta$-catenin-independent (i.e. non-canonical) Wnt/PCP and $\mathrm{Wnt} / \mathrm{Ca}^{2+}$ signaling pathways, respectively. Besides allografts I also analyzed the phosphorylation status of the proteins in the respective parental cell lines. Neither JNK nor CaMKII showed significant phosporylation compared to the positive controls indicating that both kinases are inactive both in vitro and in vivo in allografts.

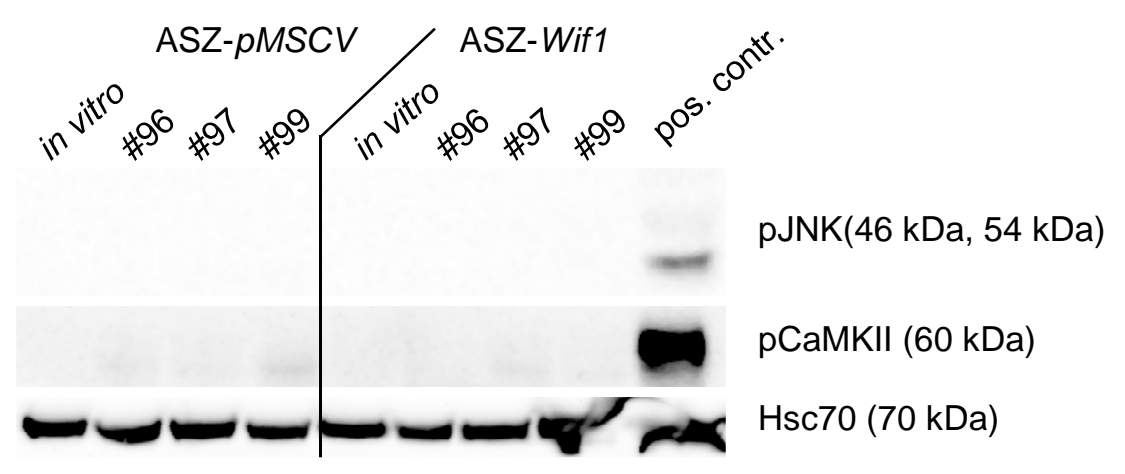

Fig. 33: Western blot of pJNK and pCaMKII in ASZ-pMSCV and ASZ-Wif1 allografts

Protein samples from ASZ-pMSCV and ASZ-Wif1 in vitro cultures (in vitro) and from allografts of the three mice \#96, 97 and 99 (\#96, 97 and 99) were analyzed. pJNK antibody detects phosphorylated SAPK/JNK (Mapk8/9) protein with the size of $46 \mathrm{kDa}$ and $54 \mathrm{kDa}$. pCaMKII antibody detects phosphorylated CaMKII protein with the size of 50 $\mathrm{kDa}$ (data not shown) and $60 \mathrm{kDa}$. Hsc70 served as loading control.

As discussed later Wif1 was described to bind several EGF receptors. For this reason, the phosphorylation status of several proteins involved in EGFR signaling was analyzed. Fig. 34 shows the phosphorylation status of Akt and of S6. S6 is a target of mTOR which in turn can be activated by Akt. As shown in Fig. 34 Akt is unphosphorylated in ASZ-pMSCV and ASZ-Wif1 in vitro samples. In contrast, the individual allograft samples showed elevated phosphorylation levels compared with in vitro samples. However, they exhibit a very heterogeneous protein expression and protein phosphorylation pattern, which was not clearly different between ASZ-pMSCV and ASZ-Wif1 derived allografts. The same was true for the Akt/mTOR downstream target 
S6. Therefore, the data suggest that Wif1 overexpression does not significantly alter Akt/mTOR signaling activity.

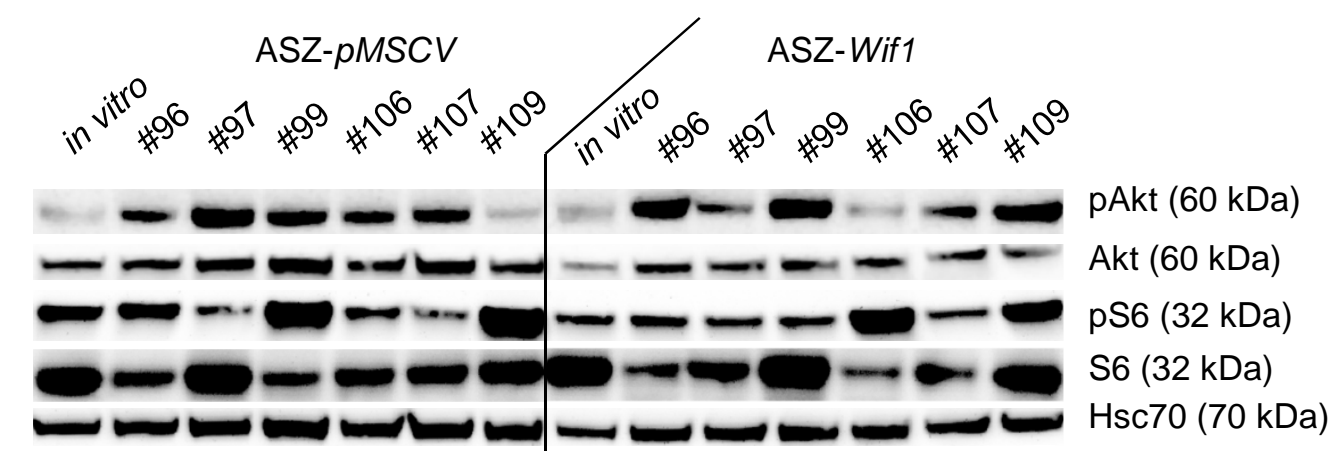

Fig. 34: Western blot of pAkt, Akt, pS6 and S6 in ASZ-pMSCV and ASZ-Wif1 allografts

Protein samples from ASZ-pMSCV and ASZ-Wif1 in vitro cultures (in vitro) and from allografts of the six mice \#96, 97, 99, 106, 107 and 109 (\#96, 97, 99, 106, 107 and 109) were analyzed. pAkt antibody detects phosphorylated Akt and Akt antibody detects total Akt protein with a size of $60 \mathrm{kDa}$. pS6 antibody detects phosphorylated S6 and S6 antibody detects total S6 protein with a size of $32 \mathrm{kDa}$. Hsc70 served as loading control. Please note that Hsc70 loading control in this Figure is identical with that in Fig. 36 and Fig. 46 as all proteins were detected using the same membrane.

Beside S6 and Akt, the additional downstream effectors of EGFR signaling PKC and Erk1/2 were examined regarding their phosphorylation status. The ASZ-Wif1 cell line (in vitro; see Fig. 35) showed an elevated phosphorylation level of PKC compared with the ASZ-pMSCV cell line. Moreover, PKC phosphorylation was maintained in ASZ-Wif1 derived allografts. In ASZ-pMSCV allografts the phosphorylation status remained at low levels comparable with the in vitro sample (see Fig. 35).

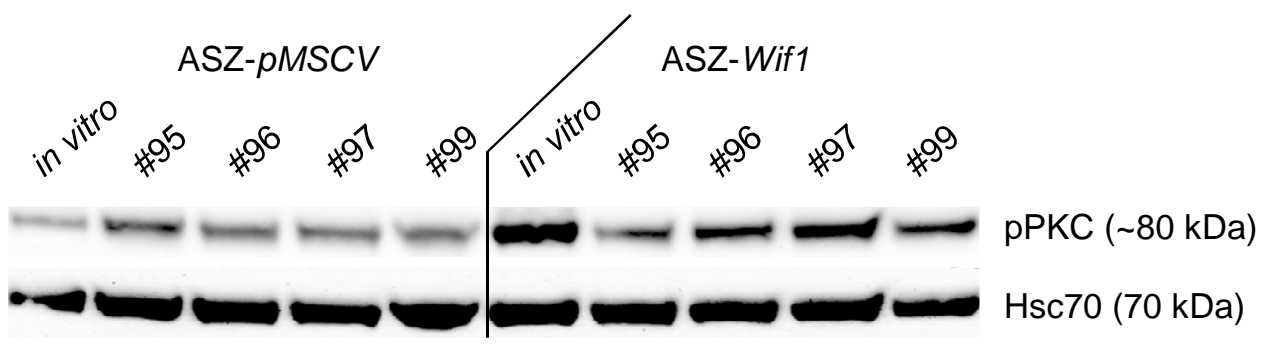

Fig. 35: Western blot of pPKC in ASZ-pMSCV and ASZ-Wif1

Protein samples from ASZ-pMSCV and ASZ-Wif1 in vitro cultures (in vitro) and from allografts of the four mice \#95, 96, 97 and 99 (\#95, 96, 97 and 99) were analyzed. Pan pPKC antibody detects phosphorylated forms of PKC protein with a size of 78-82 kDa. pErk1 and 2 antibody detects phosphorylated Erk1 and 2 protein with a size of 44 and $42 \mathrm{kDa}$, respectively. Erk1 and 2 antibody detects total Erk1 and 2 protein with a size of 44 and $42 \mathrm{kDa}$, respectively. Hsc70 served as loading control. 
The total amounts of Erk1 and Erk2 were at comparable levels in all analyzed in vitro and in vivo samples, except for ASZ-pMSCV and ASZ-Wif1 allografts from mouse \#109 showing relatively low total Erk1 and Erk2 expression levels (see Fig. 36). Moreover, the total expression of Erk2 seemed to be higher than total expression of Erk1 in all samples, provided that the antibody has equal binding affinities to both proteins. The phosphorylation of Erk2 in both in vitro samples was at comparable levels. In the allografts, the phosphorylation of Erk2 seemed to be slightly increased in most ASZ-Wif1 derived samples (\#96, 99, 106, 107 and 109) compared with ASZ-pMSCV allografts. Interestingly, the phosphorylation of Erk1 was slightly increased in the ASZ-Wif1 cell line compared to the ASZ-pMSCV cell line. When the cell lines were transplanted Erk1 phosphorylation was reduced in ASZ-pMSCV allografts but was maintained in ASZ-Wif1 allografts.

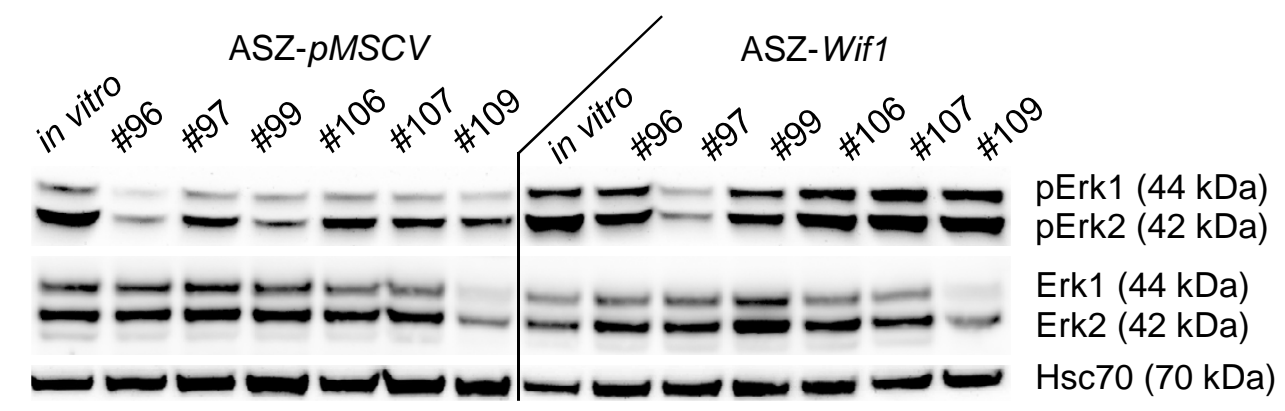

Fig. 36: Western blot of pErk1 and 2 and Erk1 and 2 in ASZ-pMSCV and ASZ-Wif1 allografts

Protein samples from in vitro cultures and from allografts (\#96-109) were analyzed. pPKC antibody detects phosphorylated forms of PKC protein with a size of 78-82 kDa. pErk1 and 2 antibody detects phosphorylated Erk1 and 2 protein with a size of 44 and $42 \mathrm{kDa}$, respectively. Erk1 and 2 antibody detects total Erk1 and 2 protein with a size of 44 and $42 \mathrm{kDa}$, respectively. Hsc70 served as loading control. Please note that Hsc70 loading control in this Figure is identical with that in Fig. 34 and with that in Fig.46 as all proteins were detected using the same membrane.

Together, these results suggest that Wif1 overexpression in ASZ001 cells results in the maintenance of PKC and Erk1/2 phosphorylation after transplantation in nude mice.

\section{IV.5.2 Cellular response on Wif1 in vivo}

As Wif1 reduced the growth of ASZ derived allografts (see Fig. 26), the differentiation status, apoptosis, the deposition of extracellular matrix, vascularization and proliferation of the tumors were analyzed. 


\section{IV.5.2.1 Effect of Wif1 on differentiation in vivo}

The expression of early and late markers for differentiation of keratinocytes was analyzed by qRT-PCR and the proportion of keratin in the allografts was measured using the cellSens software.

The markers for early differentiation, loricrin (Lor) and involucrin (IVI), as well as the markers for late differentiation, keratin 1 and 10 (Krt1 and Krt10), did not show any significant differences in expression levels when the data of 10 individual ASZ-pMSCV or ASZ-Wif1 allografts were compared (see Fig. 37).

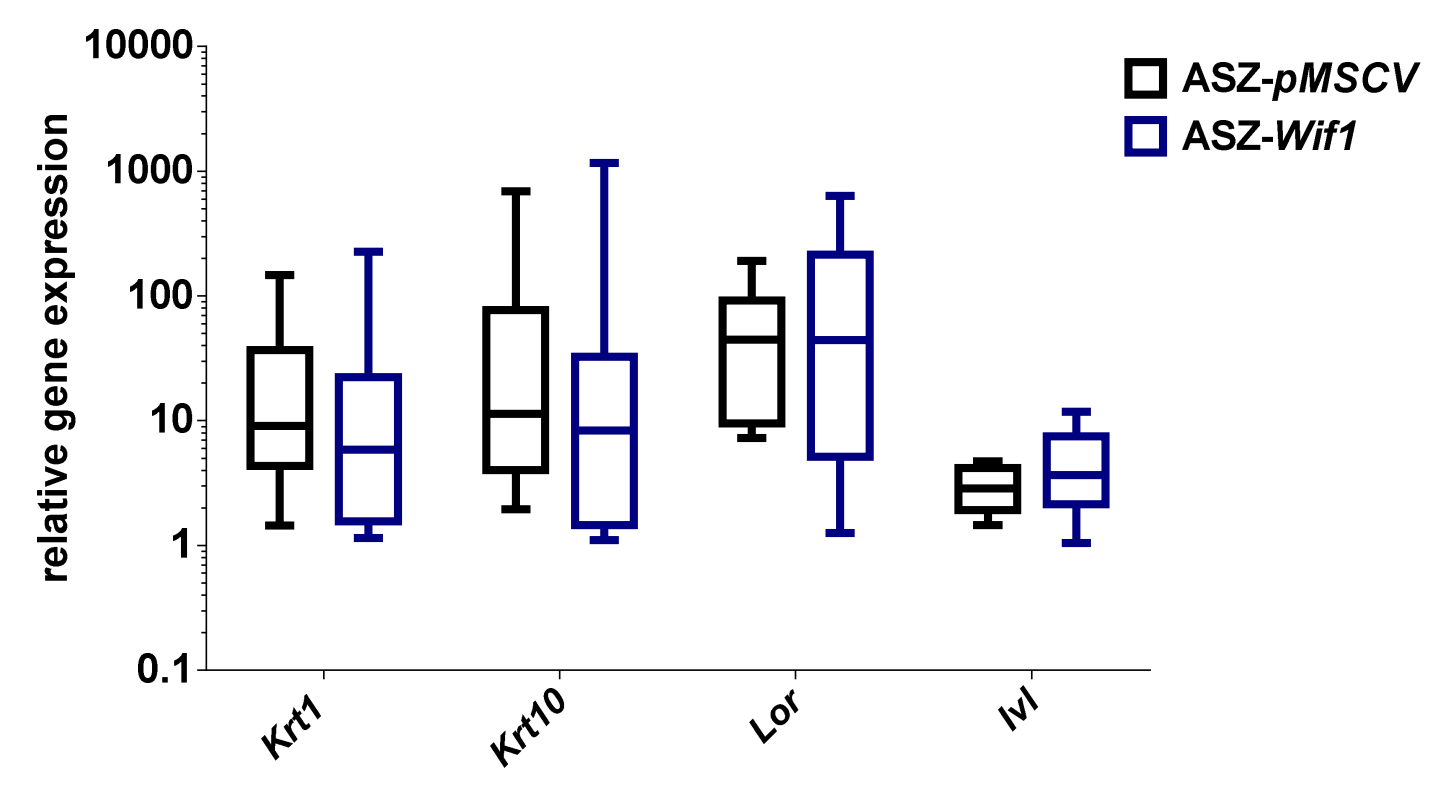

Fig. 37: qRT-PCRs of early and late keratinocyte differentiation markers in ASZ-pMSCV and ASZ-Wif1 allografts

Each bar summarizes data of 10 individual ASZ-pMSCV or ASZ-Wif1 derived tumors. Statistical significance was tested using Wilcoxon test. All data are represented as a box-whisker plot: median (horizontal line), 25-75 percentile (box), 5-95 percentile (whiskers). Statistical significance was tested using Wilcoxon test.

In order to determine the proportion of keratin in the allografts, keratinous areas were subtracted from vital tumor area of H\&E stained paraffin sections using cellSens software. For this purpose, 3 pictures each derived from either 16 ASZ-pMSCV or 16 ASZ-Wif1 tumors were analyzed. However no significant difference between allografts was detected (see Fig. 38). 


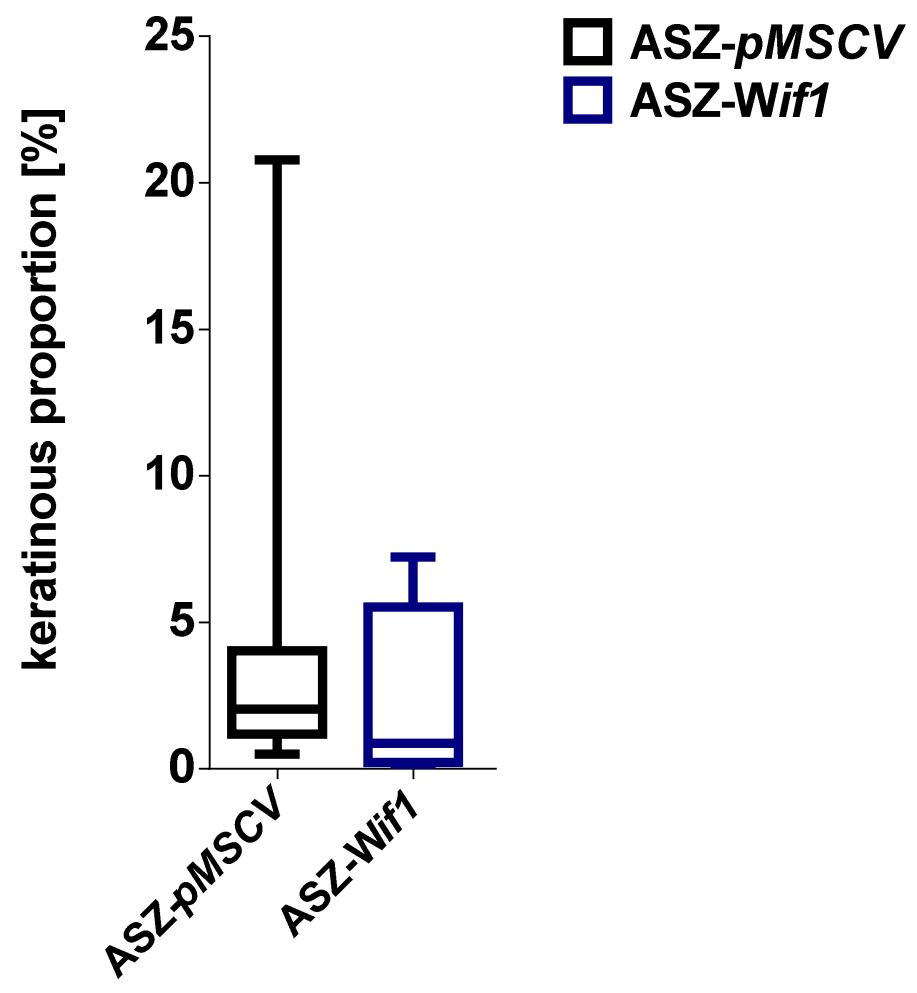

Fig. 38: Proportion of keratinous tissue in ASZ-pMSCV and ASZ-Wif1 allografts

Proportion of keratin in \% was measured in H\&E stained paraffin sections by means of cell sense software. Each plot summarizes values from 3 images each derived from 16 individual tumors. Statistical significance was tested using Mann-Whitney $U$ test. All data are represented as a box-whisker plot: median (horizontal line), 25-75 percentile (box), 5-95 percentile (whiskers). Statistical significance was tested using Wilcoxon test.

\section{IV.5.2.2 Effect of Wif1 on EMT marker gene expression in vivo}

Since Wif1 can induce a reversal of EMT in prostate cancer cells (Yee et al. 2010), the expression of Cdh1, Cdh2 and Fn1 was analyzed by means of qRT-PCR. While upregulation of Cdh1 is indicative for the induction of epithelial cell fate, Cdh2 and Fn1 expressions are associated with EMT. However, as demonstrated in Fig. 39, none of the examined transcripts was differentially expressed in ASZ-pMSCV or ASZ-Wif1 derived allografts. 


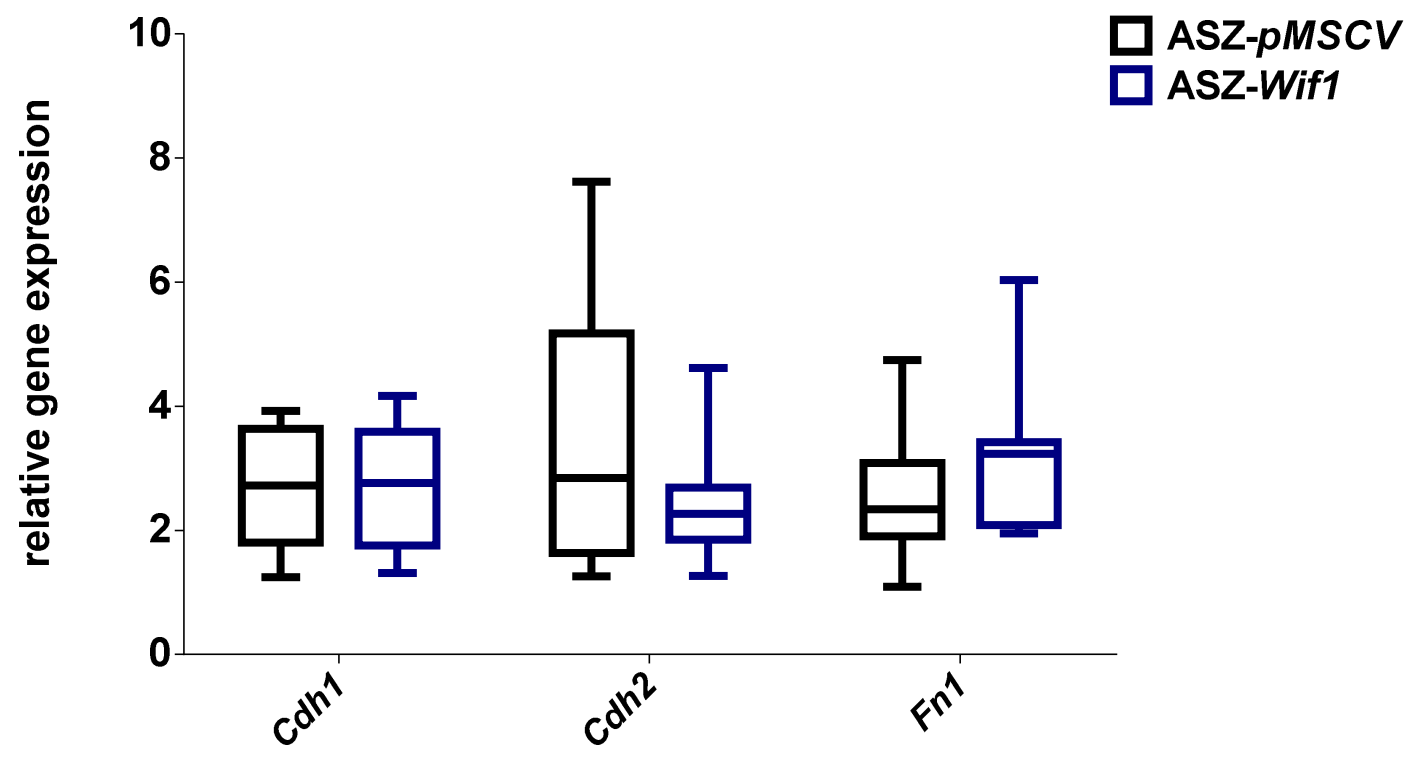

Fig. 39: qRT-PCRs of EMT markers in ASZ-pMSCV and ASZ-Wif1 allografts

Each plot summarizes values from 10 individual ASZ-pMSCV and ASZ-Wif1 allografts each. Statistical significance was tested using Wilcoxon test. All data are represented as a box-whisker plot: median (horizontal line), 25-75 percentile (box), minimum and maximum values (whiskers). Statistical significance was tested using Wilcoxon test.

\section{IV.5.2.3 Effect of Wif1 on vascularization}

Vascularization can restrict growth and size of tumors as it limits the supply with nutrients. To investigate whether Wif1 alters the vascularization of the tumors, paraffin sections were stained with an antibody against CD34, which is a marker for vascular endothelial cells. Counting the vessels per vision field revealed no significant difference between ASZ-pMSCV and ASZ-Wif1 allografts (see Fig. 40). 


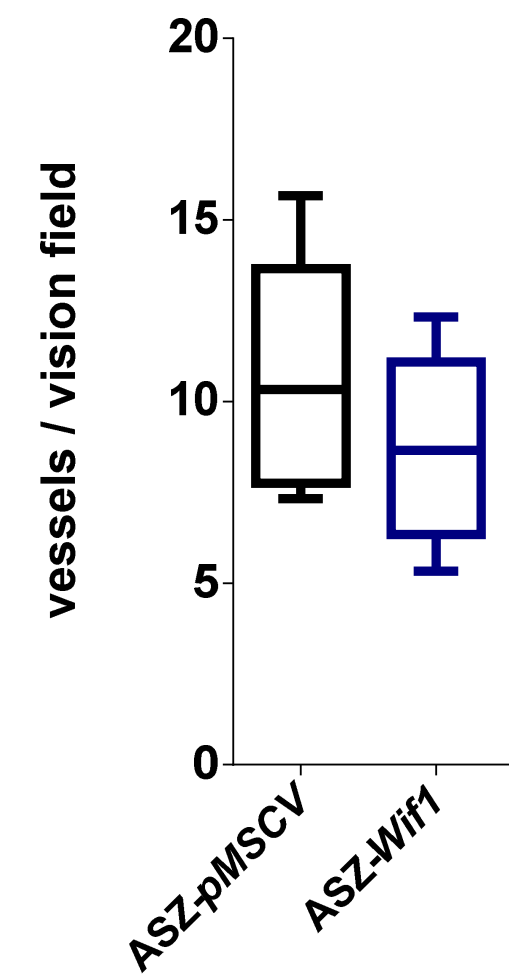

Fig. 40: Number of CD34 positive blood vessels in ASZ-pMSCV and ASZ-Wif1 allografts

Number of blood vessels was counted on paraffin sections stained with an anti CD34 antibody. Analyzed were 3 images of 8 allograft samples each. Statistical significance was tested using Mann-Whitney test. All data are represented as a box-whisker plot: median (horizontal line), 25-75 percentile (box), minimum and maximum values (whiskers). Statistical significance was tested using Wilcoxon test.

\section{IV.5.2.4 Effect of Wif1 on cell death in vivo}

Since apoptosis could have been causative for reduced growth of ASZ-Wif1 derived allografts, Western blot detecting cleaved Caspase 3 in protein lysates and TUNEL and p53 staining using paraffin sections from ASZ-pMSCV and ASZ-Wif1 allografts were performed. Cleavage of Caspase 3 and nuclear localization of p53 indicate the induction of apoptosis. Moreover, when cells undergo apoptosis, fragmentation of DNA occurs, which can be visualized by TUNEL.

When the cleavage of Caspase 3 was analyzed, no obvious differences between ASZ-pMSCV and ASZ-Wif1 allografts could be detected (see Fig. 41). Furthermore, neither the percentage of cells positive for nuclear p53 (see Fig. 42) nor TUNEL (see Fig. 43) revealed significant differences between ASZ-pMSCV and ASZ-Wif1 derived allografts. 


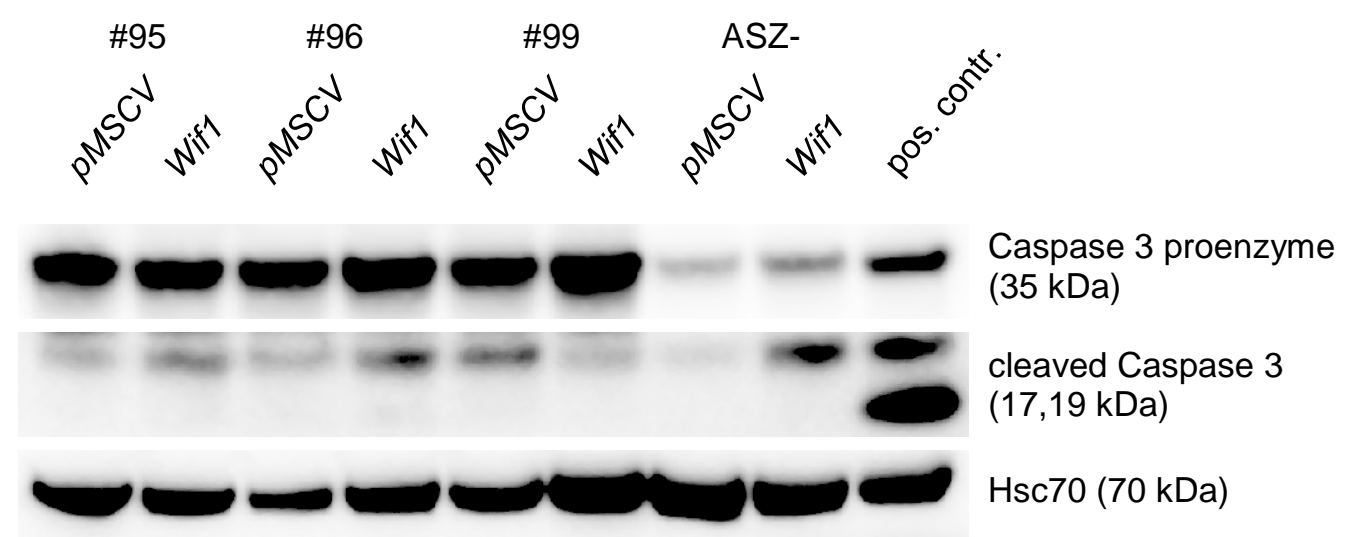

Fig. 41: Western blot of Caspase 3 in ASZ-pMSCV and ASZ-Wif1 allografts

Protein samples from in vitro cultures and from allografts (\#95, 96, and 99) were analyzed. Caspase 3 antibody detects total Caspase 3 proenzyme protein with a size of $35 \mathrm{kDa}$ and the cleaved Caspase 3 at a size of 17 and 19 $\mathrm{kDa}$. Hsc70 served as loading control.

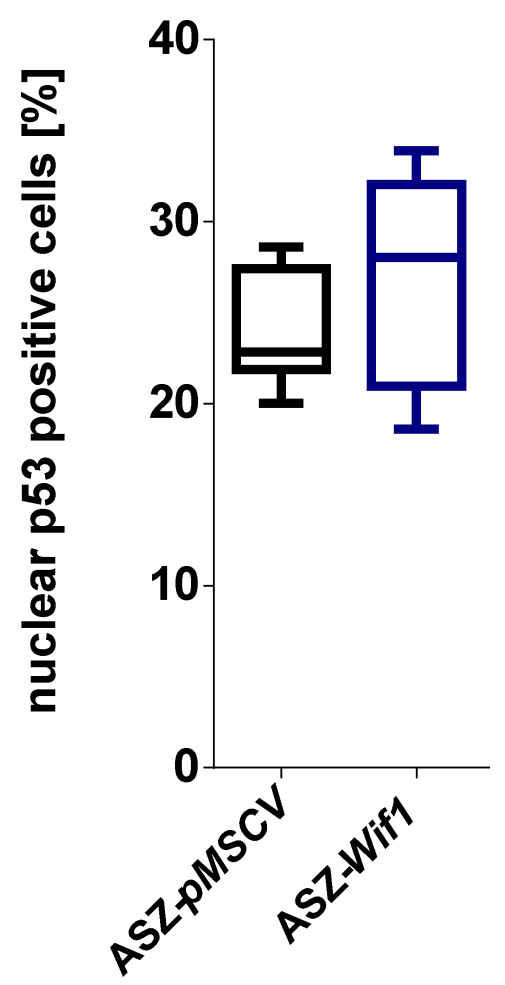

Fig. 42: Nuclear p53 staining of ASZ-pMSCV and ASZ-Wif1 allografts.

Proportion of nuclear p53 staining was calculated using the FIJl software. Analyzed were 3 images of 7 allograft samples each. All data are represented as a box-whisker plot: median (horizontal line), 25-75 percentile (box), 5-95 percentile (whiskers). Statistical significance was tested using Wilcoxon test. 


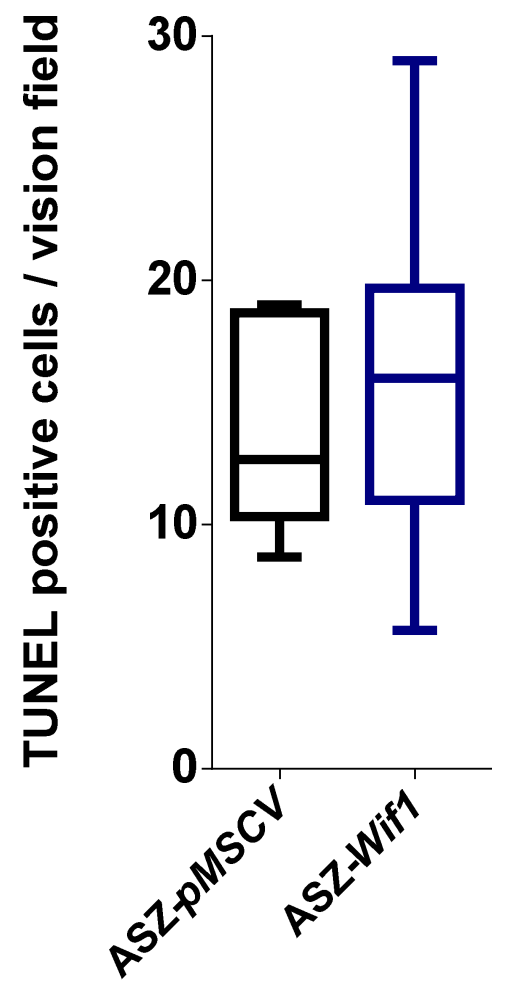

Fig. 43: TUNEL staining of ASZ-pMSCV and ASZ-Wif1 allografts

Number of positive cells staining was calculated using the Alpha View software. Analyzed were 3 images of 7 allograft samples each. All data are represented as a box-whisker plot: median (horizontal line), 25-75 percentile (box), 5-95 percentile (whiskers). Statistical significance was tested using Wilcoxon test.

Together, these data show that induction of apoptosis is not the mechanism underlying reduced tumor growth of ASZ-Wif1 allografts.

\section{IV.5.2.5 Effect of Wif1 on deposition of extracellular matrix in vivo}

Remodelling of extracellular matrix (ECM) is necessary for tumor cell migration and metastasis. However, whether ECM is protective or not is highly context specific and depends on the composition of ECM components (Honma et al. 2007; Fullar et al. 2015). To investigate whether Wif1 changes the global deposition of collagen which is a main component of the ECM, Ladewig staining of paraffin sections was performed in the Department of Neuropathology, University Medical Center Göttingen. This method results in blue coloring of collagens. Shown in Fig. 44 are examples of paraffin sections 
from ASZ-pMSCV and ASZ-Wif1 derived allografts (top row). The collagen was stained in blue and after image software based extraction of the blue channel a black and white image was generated (bottom row) allowing for automated quantification of the collagen proportion using FIJl software (see Fig. 44).

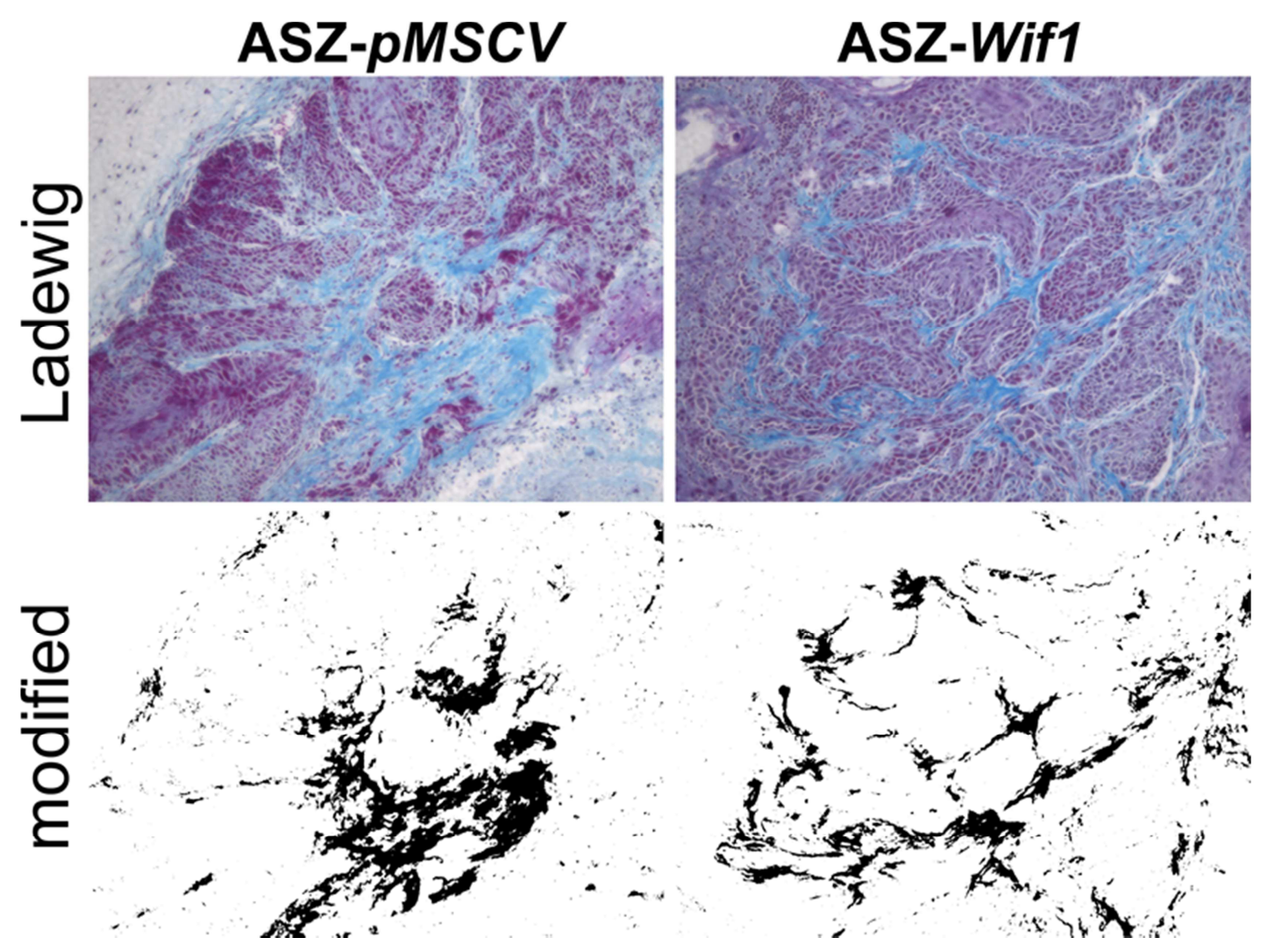

Fig. 44: Ladewig staining of ASZ-pMSCV and ASZ-Wif1 allografts

Ladewig staining of paraffin sections of ASZ-pMSCV and ASZ-Wif1 allografts was performed according to standard protocols. Collagens are stained in blue (top row). Using image software FIJI the blue channel was extracted and a black and white image was generated (bottom row).

Since the tumors also contained necrotic areas (see Fig. 28), the proportion of collagen within the vital as well as in the necrotic tumor tissue was calculated. Shown in Fig. 45 is the mean proportion of collagen per vision field and the $95 \%$ confidence interval $(\mathrm{Cl})$. In both, the viable tumor areas and necrotic areas, ASZ-Wif1 derived allografts exhibited an increased proportion of collagen. However, this was only significant for the necrotic areas. 


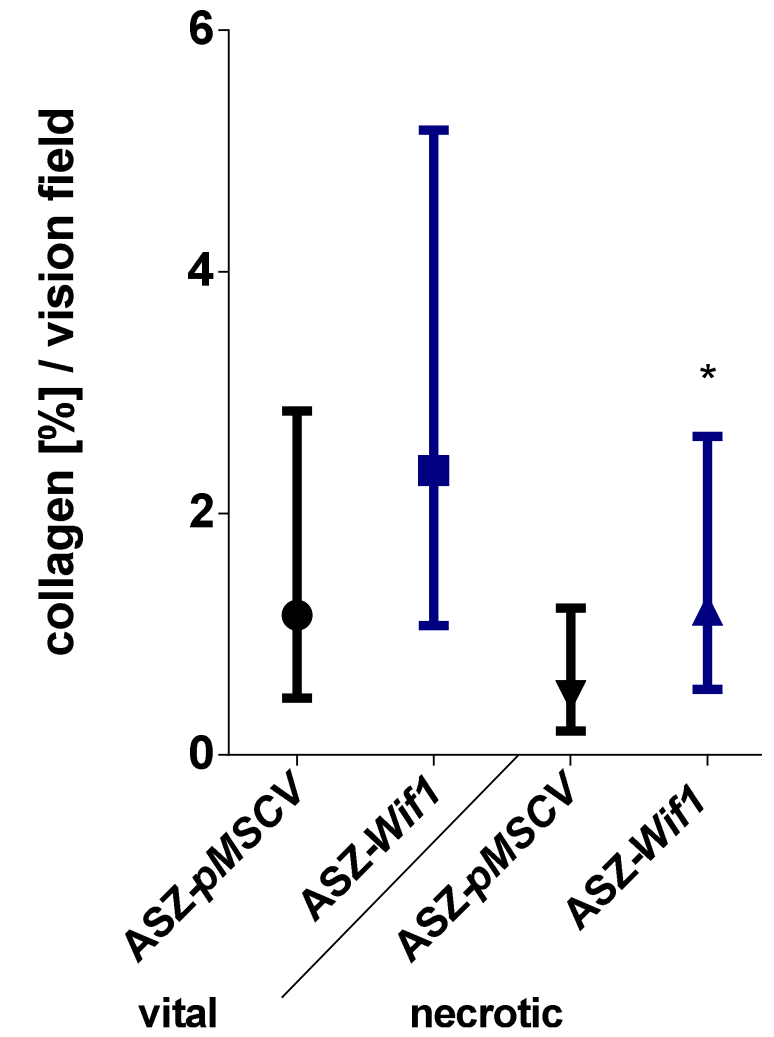

Fig. 45: Collagen proportion in ASZ-pMSCV and ASZ-Wif1 allografts

Using image software FIJI the proportion of blue stained collagen was calculated for each image. Each value represents the mean and the $95 \% \mathrm{Cl}$ of 11 tumors for which 3 images each were analyzed. Statistical significance was tested using mixed linear model. ${ }^{*} p<0.05$.

As Wif1 can bind to HSGPs in the extracellular space we speculated whether Sdc-2, a main component of HSPGs, is also affected by Wif1 overexpression. For this purpose, a Western blot was performed (see Fig. 46). In both in vitro samples no Sdc-2 expression was detectable.

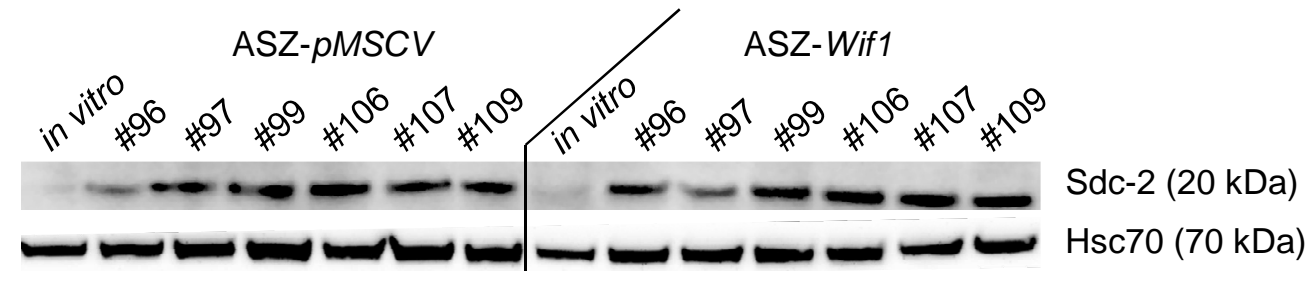

Fig. 46: Western blot of Sdc-2 in ASZ-pMSCV and ASZ-Wif1 allografts

Protein samples from in vitro cultures and from allografts (\#96-109) were analyzed. Sdc-2 antibody detects total Sdc-2 protein with a size of $20 \mathrm{kDa}$. Hsc70 served as loading control. Please note that Hsc70 loading control in this Figure is identical with that in Fig. 34 and 36 as all proteins were detected using the same membrane. 


\section{IV.5.2.6 Effect of Wif1 on proliferation in vivo}

In order to evaluate the impact of Wif1 overexpression on proliferative capacity of ASZ-pMSCV and ASZ-Wif1 derived tumors, immunohistochemical stainings of the S-phase associated marker Ki67 was performed.

ASZ-Wif1 derived allografts revealed a significantly reduced number of Ki67 positive nuclei per 1000 cells compared to ASZ-pMSCV derived allografts. This indicates that ASZ-Wif1 allografts show a lower proliferative capacity (see Fig. 47).

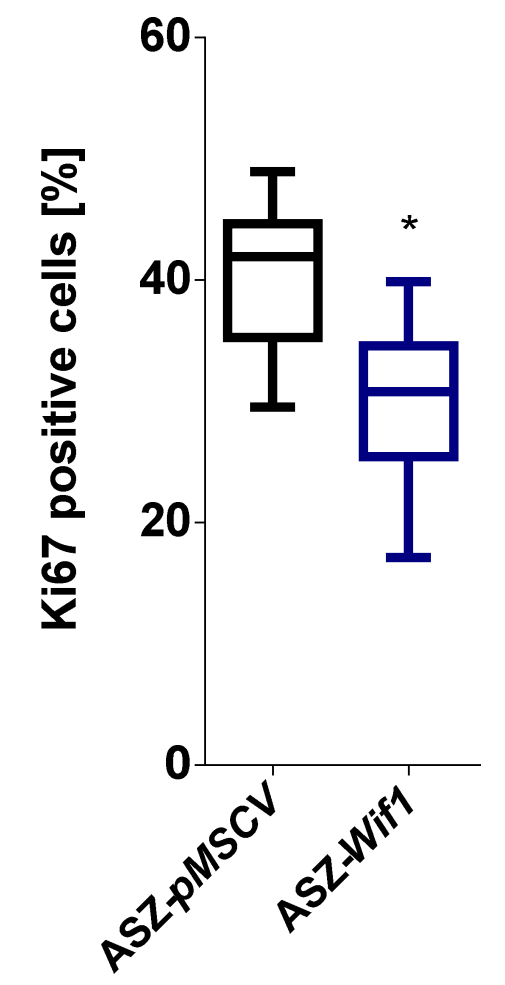

Fig. 47: Evaluation of Ki67 staining of ASZ-pMSCV and ASZ-Wif1 allografts

Total number of nuclei and Ki67 positive nuclei per paraffin section of ASZ-pMSCV and ASZ-Wif1 allografts were counted using image processing software FIJI. 3 images per section derived from 9 different tumor samples were analyzed for ASZ-pMSCV and ASZ-Wif1 derived allografts. Statistical significance was analyzed using Wilcoxon test. * $p<0.05$.

Since Wnt signaling is known to induce cyclin D1 expression (Shtutman et al. 1999; Zhang et al. 2012), which is a key regulator of cell cycle progression, we also analyzed cyclin D1 protein expression levels by means of Western blot. However, no obvious differences in the expression levels between ASZ-pMSCV and ASZ-Wif1 derived tumors were observed (see Fig. 48). 


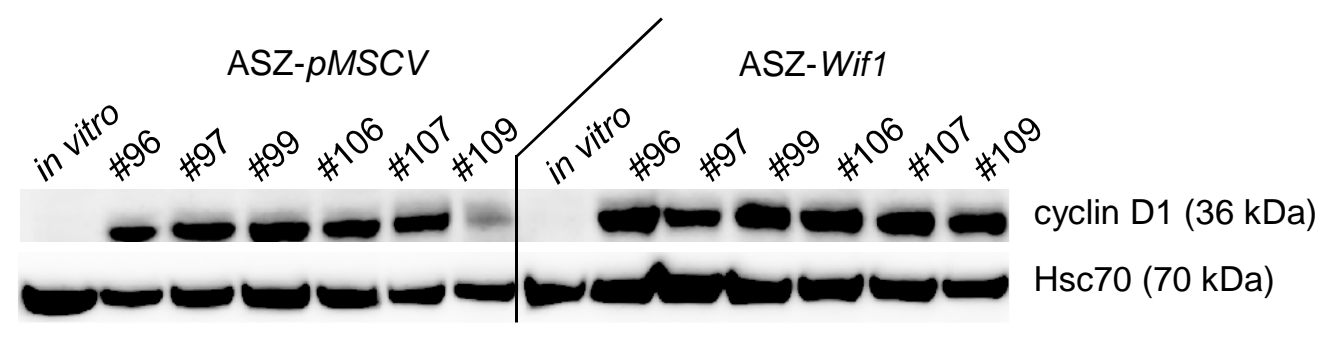

Fig. 48: Western blot of cyclin D1 in ASZ-pMSCV and ASZ-Wif1 allografts.

Protein samples from in vitro cultures and from allografts (\#96-109) were analyzed. Cyclin D1 antibody detects total cyclin D1 protein with a size of $36 \mathrm{kDa}$. Hsc70 served as loading control.

Tumor-associated macrophages (TAMs) and cancer-associated fibroblasts (CAFs) are known to affect cancer cell proliferation (Bingle et al. 2002; Augsten 2014). For example, the reduced proliferation of ASZ-Wif1 derived tumors could have been a result of decreased infiltration with TAMs or CAFs. To investigate whether the latter cellular components show a different distribution in ASZ-pMSCV and ASZ-Wif1 derived tumors, antibody staining of F4/80 and alpha smooth muscle actin (aSma) as markers for TAMs and CAFs, respectively, was performed.

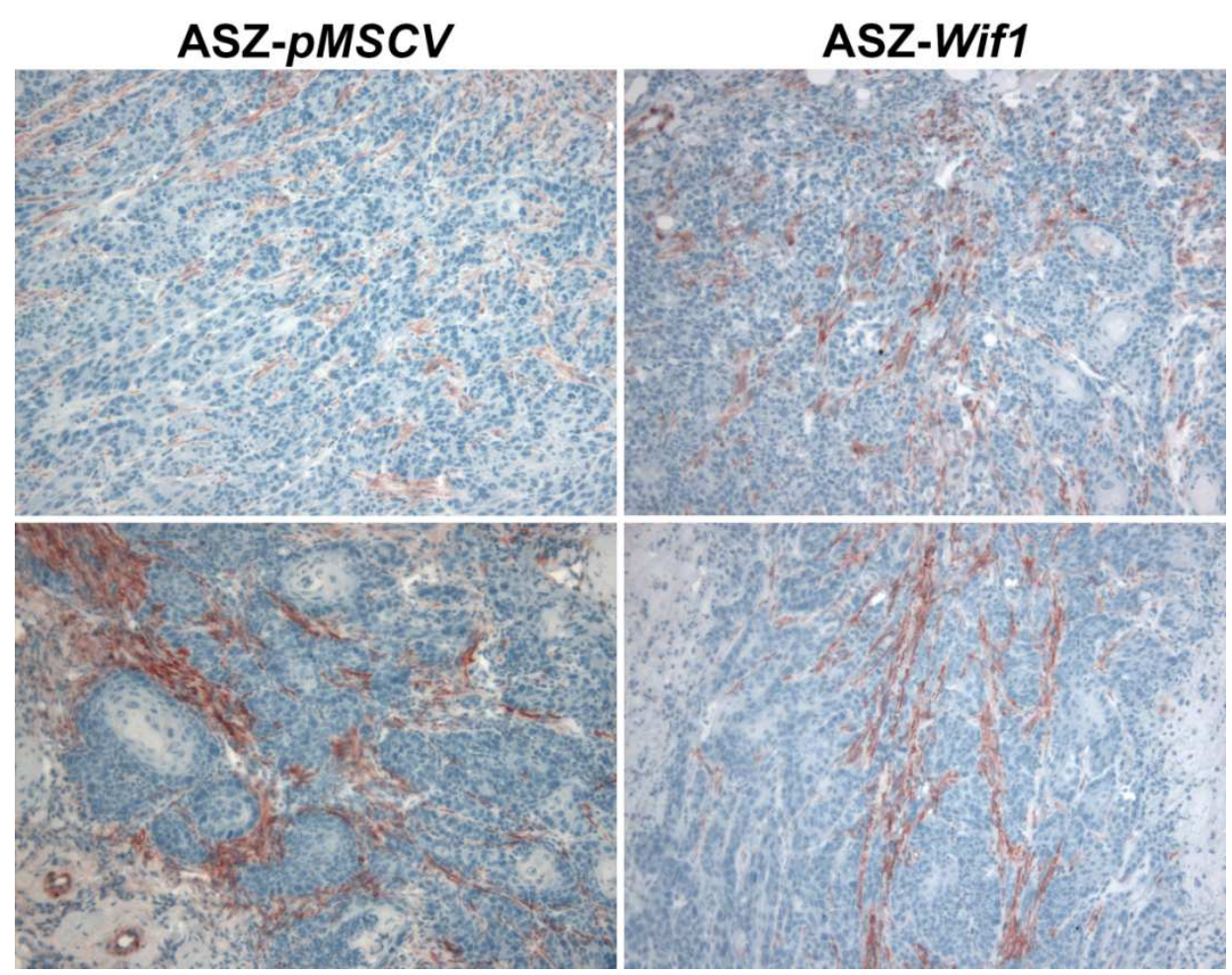

Fig. 49: antibody staining of F4/80 in ASZ-pMSCV and ASZ-Wif1 allografts

Antibody stainings of F4/80 (red) on paraffin sections derived from ASZ-pMSCV and ASZ-Wif1 allografts. Two examples each are shown. Staining reaction was performed using AEC. 
Neither the quantity of $F 4 / 80$ positive cells nor the staining pattern was obviously different between ASZ-pMSCV and ASZ-Wif1 derived allografts (see Fig. 49). Similar results were obtained with the aSma antibody that also did not show any differences concerning quantity of stained cells or staining pattern (see Fig. 50).

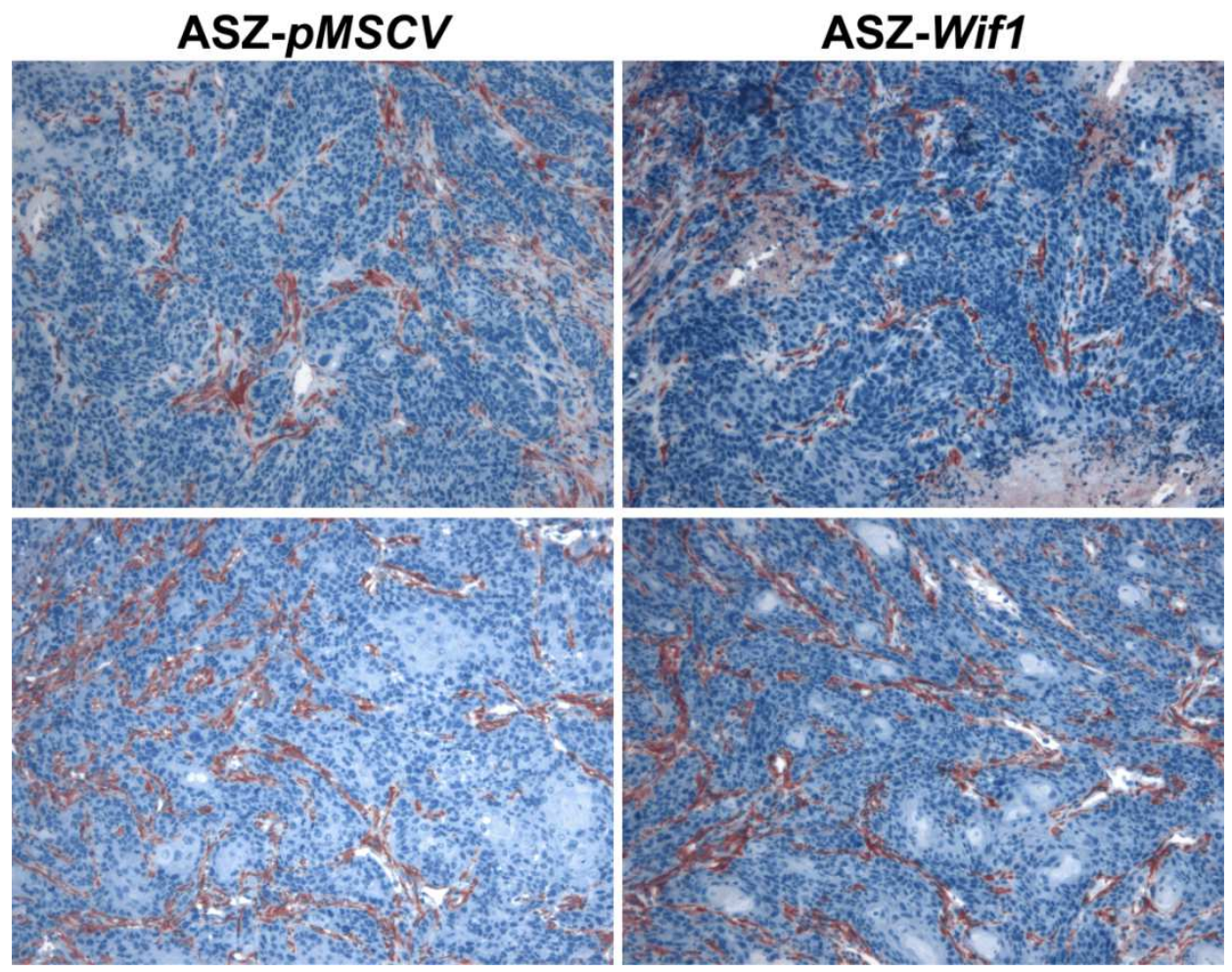

Fig. 50: Antibody staining of aSma in ASZ-pMSCV and ASZ-Wif1 allografts

Antibody stainings of aSma on paraffin sections derived from ASZ-pMSCV and ASZ-Wif1 allografts. Two examples each are shown. Staining reaction was performed using AEC.

Together these results suggest that the infiltration of the tumors with TAM and CAF is not responsible for the different growth behaviour of ASZ-pMSCV and ASZ-Wif1 derived tumors.

\section{IV.5.3 Summary of the effects of Wif1 overexpression}

Overexpression of Wif1 in the BCC cell line ASZ001 resulted in reduced tumor growth when the cells were transplanted into nude mice. This did not involve $\mathrm{Hh}$, Wnt or PI3K/Akt signaling, apoptosis, stromal composition or differentiation. Furthermore, it does not require an interaction with tumorassociated fibroblasts or macrophages. Instead, the deposition of collagen 
was increased and the proliferative capacity of the transplanted cells was reduced. This correlated with increased PKC and Erk1 phosphorylation which might indicate a putative role in EGF signaling.

\section{IV.6 Targeted disruption of Wif1 in murine BCC}

For further analysis of the function of Wif1 in vivo we employed the Ptch $^{\text {floxflox }}{ }^{\text {CreERT2 }}{ }^{T /-}$ BCC mouse model in which mice develop BCC upon tamoxifen induction. BCC of these mice express Wif1. The expression is already detectable $45 \mathrm{~d}$ after tamoxifen induced BCC development (see Fig. 52). The expression persists at least 90 days after tamoxifen injection (see Fig. 52). As in humans (see Fig. 8) the expression is detected tumor intrinsically as well as in the tumor stroma with a highly heterogeneous distribution in the latter (see Fig. 51).
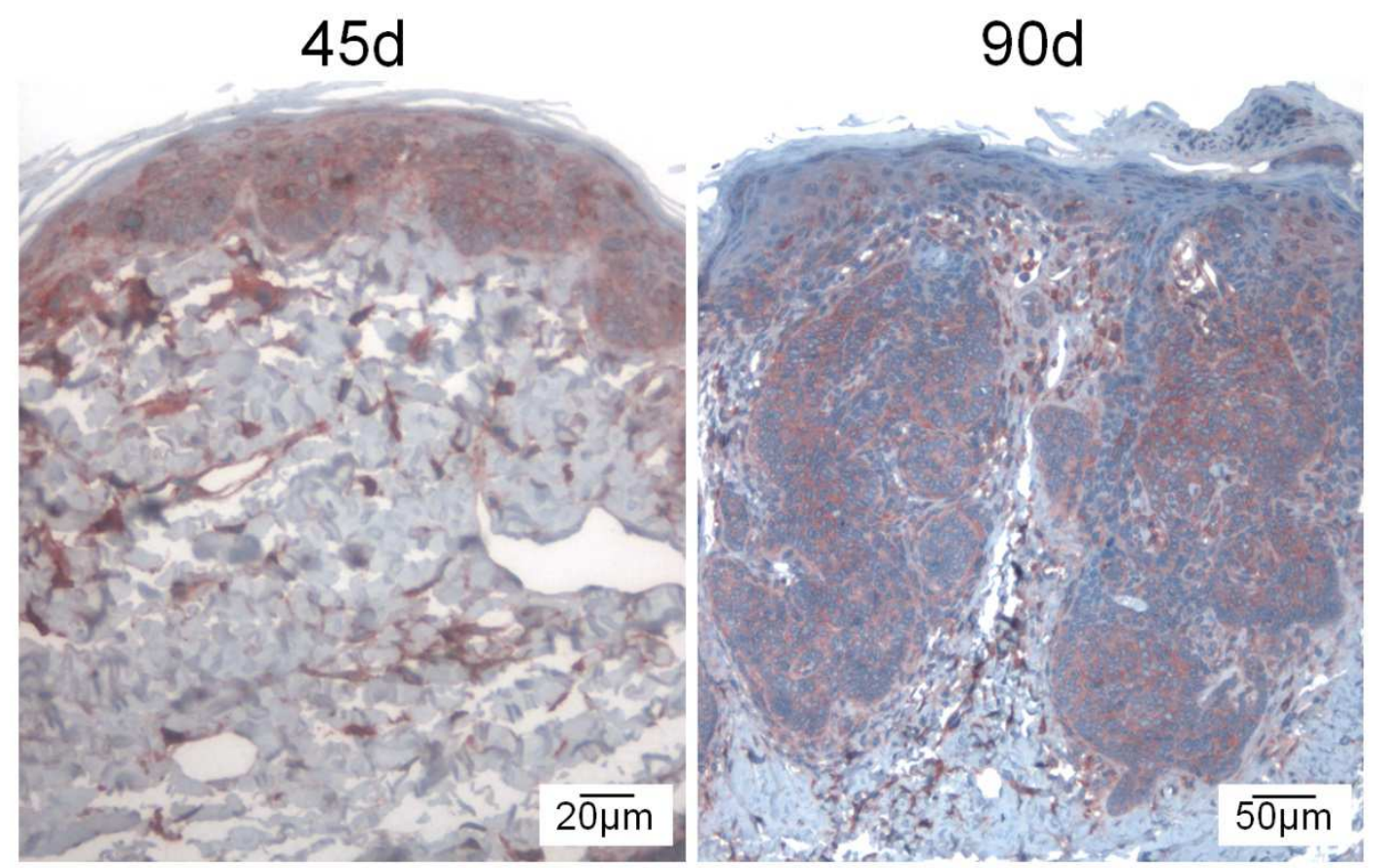

Fig. 51: Antibody staining of Wif1 in murine BCC

Anti-Wif1 antibody staining of paraffin sections derived from 45d (left) and 90d (right) BCC of Ptch ${ }^{\text {floxflox }} \mathrm{CreERT2}^{\text {T/- }}$ mice. Staining reaction was performed using AEC.

In order to investigate whether BCC growth is dependent on Wif1, Wif1 function was disrupted in BCC by breeding Ptch ${ }^{\text {flox fllox }} \mathrm{CreERT2}^{\mathrm{T} / \text { - }}$ mice with 
Wif ${ }^{-1 /}$ mice. In the Wif1 ${ }^{-1}$ mouse model a LacZ-ORF is inserted into exon 1 of the Wif1 gene which results in several in frame stop codons and thus in loss of function of Wif1. In the resulting offspring i.e. Ptch ${ }^{\text {floxflox}} \mathrm{CreERT2}^{T /-}$ Wif1 ${ }^{-1-}$ and $\mathrm{PtCh}^{\text {floxfllox}} \mathrm{CreERT2}{ }^{\mathrm{T} / \mathrm{BCC}}$ were induced by tamoxifen injection at an age of 8 weeks. Both Ptch ${ }^{\text {floxflox }}$ CreERT2 ${ }^{T /-W i f 1^{-1 /}}$ and Ptch ${ }^{\text {floxflox }}$ CreERT2 ${ }^{\text {T/ }}$ mice were randomized in 2 cohorts. In the first cohort BCC were analyzed 45 days after tamoxifen injection (45d) (Nitzki et al. 2010). The second cohort was analyzed 90 days after BCC induction. Wif1 wild-type littermates i.e. $\mathrm{PtCh}^{\text {floxflox }} \mathrm{CreERT2}^{\mathrm{T/}-}$ and littermates lacking Cre-recombinase i.e. $\mathrm{PtCh}^{\text {floxflox}}$ Wif $^{-1-}$ served as controls. None of the littermates lacking Crerecombinase that were treated with tamoxifen developed BCC. In contrast, all Ptch $^{\text {floxfllox} C r e E R T 2^{T /-} \text { and Ptch }}{ }^{\text {floxflox CreERT2 }}{ }^{\text {T/ }}$ Wif1 ${ }^{-/}$animals developed tumors. Macroscopically no difference between both BCC backgrounds was detectable. As expected Wif1 protein was not detected in skin samples from Ptch $^{\text {flox/flox}}$ CreERT2 ${ }^{T /}$ Wif1 ${ }^{-/}$animals (see Fig. 52).

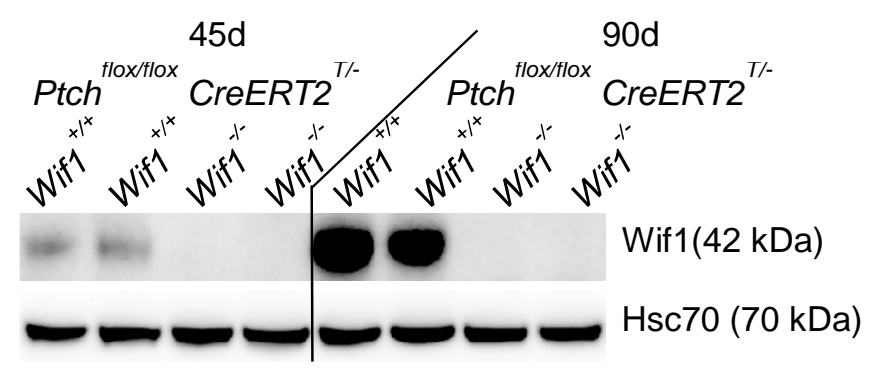

Fig. 52: Western blot of Wif1 in murine BCC

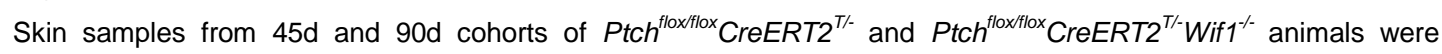
analyzed regarding Wif1 protein expression.

To assess the tumor load of Ptch ${ }^{\text {flox } f l o x} \mathrm{CreERT2}{ }^{\mathrm{T} / \mathrm{C}}$ and Ptch $^{\text {floxflox }}{ }^{-}$reERT2 ${ }^{T /}$ Wif $^{-1 /}$ animals the tumor size was measured on $\mathrm{H} \& \mathrm{E}$ stained paraffin sections ( 3 images from sections of 5 different animals each) using cellSens software. As demonstrated in Fig. 53 BCC of mice lacking Wif1 expression had the same size compared with Wif1 wild-type littermate $45 \mathrm{~d}$ after induction. However, in 90 day cohorts the size of BCC in mice lacking Wif1 expression was unambiguously larger compared with Wif1 wild-type littermates (see Fig. 54). 


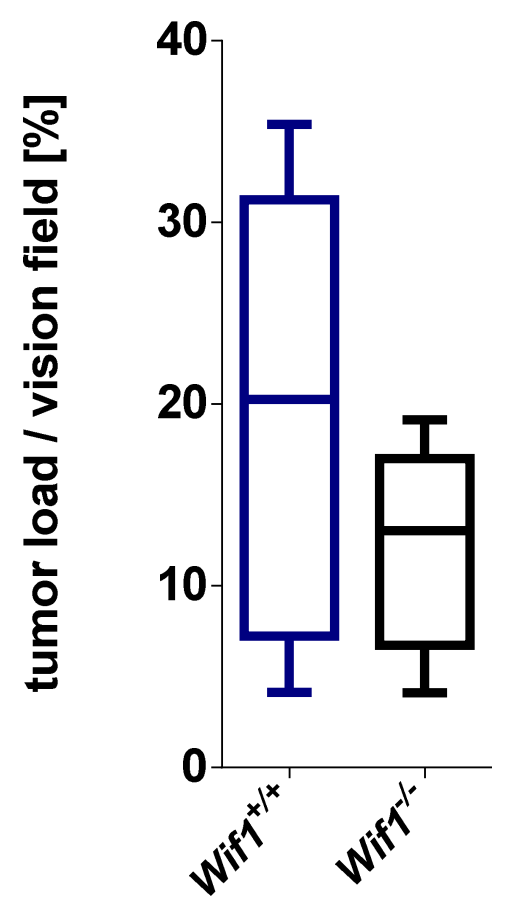

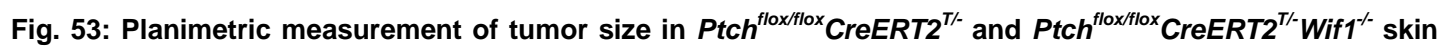
samples $45 \mathrm{~d}$ after induction

3 images of each 5 Skin samples from Ptch floxflox $C r e E R T 2{ }^{T /-}$ and $\operatorname{Ptch}^{\text {floxfllox}}$ CreERT2 ${ }^{T /}$ Wif $1^{-/}$animals were analyzed using software cellSens.

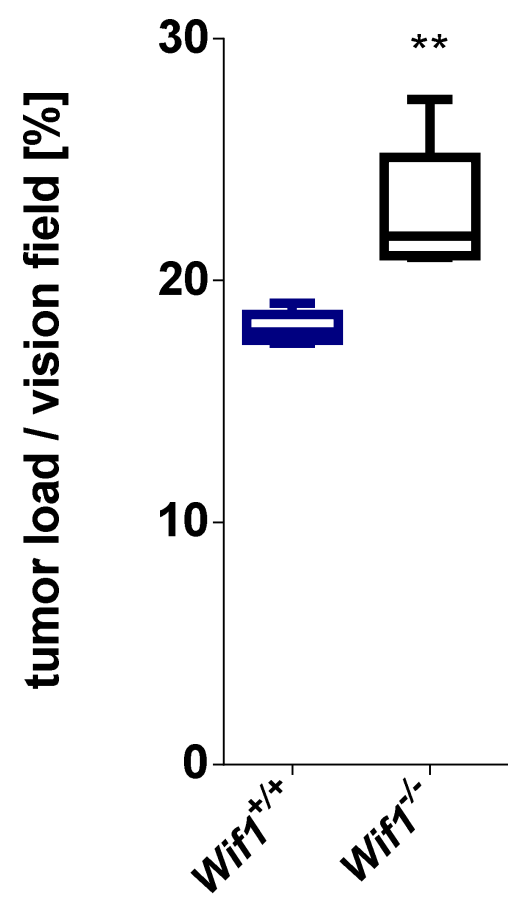

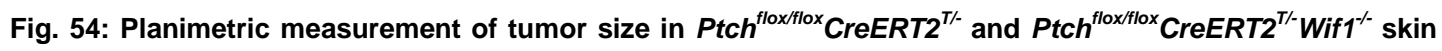
samples $90 \mathrm{~d}$ after induction

3 images of each 5 Skin samples from Ptch ${ }^{\text {floxflox }}$ CreERT2 ${ }^{T /-}$ and $\operatorname{Ptch}^{\text {floxfllox}}$ CreERT2 ${ }^{T /}$ Wif $1^{-1 /}$ animals were analyzed using software cellSens. 
Next, in order to investigate the proliferative capacity of the tumors, an anti-Ki67 antibody staining was performed on paraffin embedded sections. As shown in Fig. 55 the percentage of Ki67 positive cells was significantly higher on a Wif1 deficient background in both, the $45 \mathrm{~d}$ and $90 \mathrm{~d}$, cohorts.

Together, these data indicate that in accordance with the allograft experiments (see Fig. 26) Wif1 is a factor that is neccessary to restrict BCC growth.

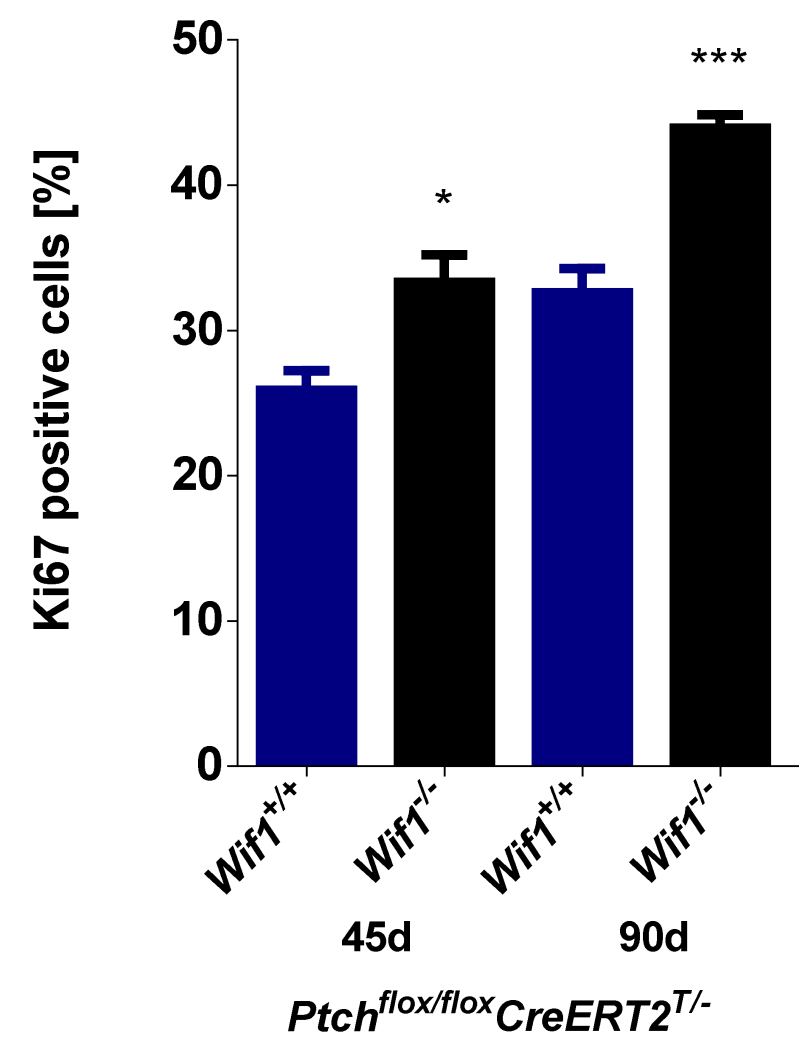

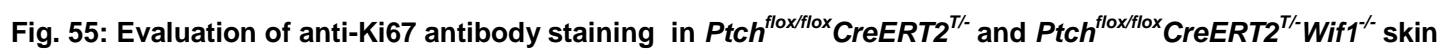
samples $45 \mathrm{~d}$ and $90 \mathrm{~d}$ after induction

Ki67 positive cells were counted using AlphaView software. 3 images of 3 skin samples each were analyzed. Exclusively BCC cells were counted. All data are represented as a mean + SEM. Statistical significance was evaluated using Mann-Whitney $U$ test. ${ }^{*} p<0.05,{ }^{* * *} p<0.001$. 


\section{Discussion}

\section{V.1 WIF1 in human tumors of the skin}

As shown by antibody staining, WIF1 is highly expressed in human and also murine BCC. Indeed, all analyzed human BCC samples exhibited tumorintrinsic WIF1 expression. This is in line with data from the human protein atlas webpage where 5 out of 6 samples from BCC patients were described to express at least weak WIF1 protein levels in antibody stainings.

Using immortalized rat kidney cells it was found that GLI1 transduction induced the expression of Wif1 (Louro et al. 2002). Because at that time WIF1 was already known as a potential tumor suppressor gene, the authors already speculated that WIF1 expression may be responsible for the infrequent occurrence of metastases in BCC patients (Louro et al. 2002). Even though this speculation was not formally proven, the authors proposed that a Wif1 knockout could demonstrate its tumor suppressive function in skin cancer. In line with this assumption, WIF1 was found to be downregulated in melanomas compared with nevi (Haqq et al. 2005). Taken together, this drove us to hypothesize that Wif1 might keep BCC semi-malignant and prevents it from malignant transformation. We also speculated that WIF1 is absent or present at significantly lower levels in invasive BCC, SCC and malignant melanomas compared to indolent-growth subtypes of BCC. However, as shown by our data this was not the case.

In our experiments we used qRT-PCR to quantify WIF1 expression in indolent-growth and invasive variants of BCC, SCC and malignant melanomas. Whereas antibody staining does not allow comparative quantification of gene expression in different tumor samples and entities, qRT-PCR does. Thus, laser microdissection of paraffin-embedded tumor tissue was performed and WIF1 mRNA levels were analyzed. WIF1 expression in invasive BCC, SCC and melanomas was at comparable levels and expression in SCC and melanomas was significantly higher compared to the indolent-growth variants of BCC, i.e. superficial and nodular BCC. Although this contradicted our working hypothesis that WIF1 is a tumor suppressor in BCC and as such should be expressed at higher levels in 
indolent-growth subtypes of BCC when compared to more malignant skin tumors, our consecutive data showed that Wif1 inhibited BCC growth in mouse models. This leaves several possibilities for interpretation: First, Wif1 exerts its tumor suppressive functions only in the mouse. However, this is unlikely since there is only one report suggesting an oncogenic function of WIF1, whereas all other studies unambiguously showed that WIF1 acts as a tumor suppressor in diverse human tumor entities. Furthermore, in melanoma WIF1 gene transfer was shown to inhibit tumor growth in a xenograft model by suppressing proliferation (Lin et al. 2007). This supports the hypothesis of WIF1 being a tumor suppressor also in malignant skin tumors. Second, WIF1 could also have distinct functions in BCC, SCC and melanoma. For example, WIF1 promotes melanogenesis in melanocytes (Park et al. 2014) suggesting a distinct function in melanomas compared with BCC and SCC. Third, I only have determined WIF1 expression on mRNA level ignoring possible post-transcriptional, translational and posttranslational regulatory mechanisms.

Another aspect about WIF1 expression in BCC is the following: Although invasive BCC express higher WIF1 levels than indolent-growth subtypes the differences of WIF1 expression were statistically not significant. This might be different if a larger sample set would have been analyzed. In case this would be true, there would be an explanation to it: GLI1 was shown to induce Wif1 expression in rat kidney cells (Louro et al. 2002). Furthermore, Gli1 is the effector driving tumor formation in BCC (Nilsson et al. 2000). Thus elevated GLI1 activity in invasive BCC could be the cause for elevated WIF1 expression levels. However, so far this is pure speculation because GL/1 mRNA levels in invasive versus indolent-growth variants of BCC have not been quantified. Prospectively, this experiment will be conducted in our lab using the samples from the microdissections.

Noteworthy, beside tumor-intrinsic expression WIF1 was obviously also highly expressed in cells of inflammatory infiltrates and in fibroblasts of the tumor stroma (see Fig. 8). WIF1 expression in fibroblasts of the skin has been previously reported to be involved in melasma development (Kim et al. 2013). In contrast, the role of WIF1 in inflammation of the skin is currently absolutely unknown. However, it was shown that abrogation of Wnt secretion 
in keratinocytes resulted in infiltration of immune cells and inflammation of the skin (Augustin et al. 2013). This might also suggest an implication of WIF1 in the promotion of inflammation by inhibiting Wnt signaling.

Thus, WIF1 seems to exert a variety of functions in the skin and it cannot be ruled out that tumor-extrinsic WIF1 also affects BCC development. However, in this thesis I will focus on the putative tumor suppressive function of tumorintrinsic WIF1 as this appears to be a general phenomenon that applies for a variety of different cancer entities.

\section{V.2 Wif1 overexpression in the BCC cell line ASZ001}

ASZ001 is the only available BCC cell line derived from $\mathrm{Ptch}^{+/}$mice. Even though derived from a tumor that exhibited Wif1 expression, this cell line expresses Wif1 at low to undetectable levels (data not shown). This is in line with lost WIF1 expression in human cultured proliferating keratinocytes (Schluter et al. 2013). Thus, a Wif1 overexpression plasmid was generated. Using the stably transduced cell line ASZ-Wif1 the effect of Wif1 on BCC growth was examined.

\section{V.2.1 Validation of Wif1 overexpression, secretion and functionality}

Effective Wif1 overexpression in ASZ-Wif1 cells was shown on RNA as well as protein level (see Fig. 12 and Fig. 13). Since Wif1 is a secreted protein exhibiting its function in the extracellular space by sequestration of Wnt ligands, its secretion was analyzed by concentrating the medium of ASZ-Wif1 and subsequent Western blot analysis. In fact, Wif1 is secreted by ASZ-Wif1 into the medium (see Fig. 14). Unfortunately, I was not able to analyze the functionality of the secreted Wif1 by TOP/FOP reporter assay when the parental cell line ASZ001 was used. This was due to the fact that neither Wnt3a nor an active form of $\beta$-catenin induced TOP activity in this cell line. 
TOP activity is dependent on functional Lef1 which binds to Tcf/Lef binding sites of the TOP vector when activated by $\beta$-catenin. Thus, it is possible that ASZ001 simply does not express a functional form of Lef1. Alternatively, the transduction efficiency was insufficient to facilitate luciferase expression. Therefore, HEK-293 cells were employed as an alternative. In this experimental setup concentrated supernatant from ASZ-Wif1 significantly inhibited the action of Wnt3a conditioned medium as measured by the TOP/FOP reporter assay. This demonstrated that functionally active Wif1 was successfully overexpressed and secreted by ASZ-Wif1 cells. Therefore, ASZ-Wif1 cells served as basis for subsequent investigations on the role of Wif1 in BCC.

\section{V.2.2 Effects of Wif1 overexpression on the BCC cell line ASZ001}

The effect of Wif1 overexpression on BCC cell growth was analyzed in vitro and in vivo. For in vivo analyses the cell lines ASZ-Wif1 and ASZ-pMSCV were subcutaneously transplanted in the flanks of nude mice.

Similarly to xenografts of melanoma, kidney cancer and cervical cancer cells (Lin et al. 2007; Kawakami et al. 2009; Ramachandran et al. 2012) the overexpression of Wif1 resulted in reduced BCC growth (see Fig. 26) which was also reflected by reduced tumor weight (see Fig. 27). Reduced tumor growth can be a result of alterations in diverse cellular processes. Therefore, I examined the effect of Wif1 on signaling pathway activity, cell viability, apoptosis, differentiation, EMT, migration, cell cycle regulation, proliferation, and on the tumor microenvironment.

\section{V.2.2.1 Effect of Wif1 on Wnt, Hh and EGFR signaling pathways}

It has been shown that shifted, the Drosophila orthologue of Wif1, increased stabilization and diffusion of $\mathrm{Hh}$ (Glise et al. 2005) and promoted Hh signaling 
mediated by $\mathrm{Hh}$ co-receptors interference hedgehog (Ihog) and brother of Ihog Boi (Avanesov and Blair 2013). Furthermore, overexpression of Wif1 in hematopoietic stem cells induced Shh expression resulting in Hh pathway activation (Schaniel et al. 2011). As these results suggested that Wif1 may also induce $\mathrm{Hh}$ signaling in $\mathrm{BCC}$, $\mathrm{Hh}$ signaling activity was determined by Gli1 qRT-PCR in ASZ-Wif1 and ASZ-pMSCV cells. However, overexpression of Wif1 did not alter Gli1 gene expression neither in vitro nor in vivo (see Fig. 17 and 31). Although ASZ001 were derived from BCC that had activated $\mathrm{Hh}$ signaling, they express relatively low Gli1 mRNA levels (So et al. 2006). Thus, we would have expected an upregulation of Gli1 expression if Wif1 would have been able to activate $\mathrm{Hh}$ signaling. Taken together, it can be hypothesized that ASZ001 either i) lacks required coreceptors, ii) is not able to upregulate Shh or iii) is insensitive towards Shh due to its Ptch mutation. On the contrary, it has been shown that Wif1 is downstream of Gli1 as GL/1 transduction induced Wif1 expression in rat kidney cells (Louro et al. 2002). Moreover, the Wif1 promoter region contains a GLI-kruppel binding site as a regulatory element (Reguart et al. 2004), suggesting that Wif1 is downstream and not upstream of activated $\mathrm{Hh}$ signaling in BCC. If this is also true for Wif1 expression in ASZ001 it consequently did not alter Hh signaling in ASZ-Wif1 cells and allografts.

As a Wnt inhibitor Wif1 can bind to several Wnts and prevent them from binding to their receptors. Activated canonical Wnt signaling leads to a translocation of $\beta$-catenin into the nucleus and ultimately to the induction of target gene expression including Axin2 and $c-M y c$. In order to analyze whether the overexpression of Wif1 is sufficient to inhibit canonical target gene expression, the expression of Axin2 and $c-M y c$ was analyzed by qRT-PCR. In addition, the subcellular localization of $\beta$-catenin was estimated by antibody staining. No changes in the target gene expression and no nuclear $\beta$-catenin were detectable (see Fig. 17, 31 and 32). Although nuclear $\beta$-catenin is currently debated as a reliable read-out for active canonical Wnt signaling, absence of nuclear $\beta$-catenin in combination with the unaffected target gene expression strongly suggests that canonical Wnt signaling is inactive in the ASZ001-derived cell lines and respective allografts. Thus, it is 
possible that Wif1 overexpression was not able to further decrease the already very low levels of Axin2 and c-Myc.

Next, the activation status of $\mathrm{Wnt} / \mathrm{Ca}^{2+}$ and $\mathrm{Wnt} / \mathrm{PCP}$ pathways were determined as Wif1 is able to bind and inhibit several Wnts that are implicated in activation of non-canonical Wnt signaling (e.g. Wnt4, 5a, 7a and 11) (Surmann-Schmitt et al. 2009; Vassallo et al. 2015). However, JNK as mediator of active Wnt/PCP pathway was unphosphorylated. This rather suggests inactive Wnt/PCP signaling. Furthermore, the cytoplasmic $\mathrm{Ca}^{2+}$ levels of ASZ-pMSCV and ASZ-Wif1 as an indicator for activated Wnt/Ca ${ }^{2+}$ signaling were examined. Neither the the basal $\mathrm{Ca}^{2+}$ concentrations nor the maximum $\mathrm{Ca}^{2+}$ flux ratio was altered in ASZ-Wif1 (see Fig. 18). This indicates that $\mathrm{Wnt} / \mathrm{Ca}^{2+}$ signaling is either not affected by Wif1 or that the sensitivity of this assay does not allow for discrimination between small differences in $\mathrm{Ca}^{2+}$ concentrations. Moreover, CaMKII was unphosphorylated in allografts and in in vitro samples supporting that $\mathrm{Wnt} / \mathrm{Ca}^{2+}$ signaling is not affected.

However, I found PKC phosphorylation to be upregulated in vitro and to be maintained in ASZ-Wif1 allografts. The antibody detects PKC isoforms $\alpha, \beta I$, $\beta I I, \delta, \varepsilon, \eta$ and $\theta(78-85 \mathrm{kDa})$ when phosphorylated at serine 660 . Due to the minor differences in the size of the isoforms they cannot be discriminated by Western blot analysis. However, PKC $\alpha, \beta$ and $\delta$ isoforms are expressed in keratinocytes and BCC (Neill et al. 2003; Breitkreutz et al. 2007). Interestingly, loss of PKC $\alpha$ is associated with enhanced tumor growth in BCC (Neill et al. 2003). Previous results of our group showed that an antibody detecting $\alpha$ and $\beta I I$ isoforms recapitulated pan-PKC expression in BCC samples (Carstens 2010). Thus, it is highly probable that the detected PKC was constituted of $\alpha$ and $\beta$ II isoforms.

The aspect about PKC phosphorylation is of particular interest as PKC is not only involved in $\mathrm{Wnt} / \mathrm{Ca}^{2+}$ signaling but also mediates EGFR (Fan et al. 2009). As Wif1 contains 5 EGF-like domains, all high-affinity ErbB ligands contain an EGF-like domain (Yarden and Sliwkowski 2001) and since $\mathrm{Ca}^{2+}$ was not affected in ASZ-Wif1 cell lines, it is tempting to speculate that Wif1 
might also have affected EGFR signaling. Indeed, one investigation identified Wif1 as a binding partner for ErbB2, 3 and 4 that are isoforms of the EGFR (Curak 2010). Since BCC usually express EGFR (Eberl et al. 2012) and because EGFR activity can result in activation of Akt and Ras signaling the phosphorylation status of Akt, S6 and Erk1/2 was analyzed. Akt and S6 display an inconsistent phosphorylation pattern, which however did not significantly vary between ASZ-Wif1 and ASZ-pMSCV allografts. Erk1 and 2 were both phosphorylated und thus activated when the cell lines were cultured in vitro with slightly increased phosphorylation of Erk1 in ASZ-Wif1. Interestingly, when the cell lines were transplanted in nude mice, the phosphorylation status of Erk2 remained at equal levels in both cell lines. In contrast, Erk1 phosphorylation was only maintained in the ASZ-Wif1 allografts whereas it vanished in ASZ-pMSCV allografts.

Firstly, these data may indicate that Erk1 phosphorylation in the in vitro situation is fostered by specific growth factors provided by FCS that is a supplement of the culture medium. The fact that Erk1 phosphorylation was maintained exclusively in ASZ-Wif1 allografts could be a result of the concomitantly observed maintenance of PKC phosphorylation. Moreover, it could be part of activated $\mathrm{Wnt} / \mathrm{Ca}^{2+}$ signaling or active EGFR signaling (see above) (Kolch 2005; Mendoza et al. 2011). Whether the maintenance of PKC and Erk1 phosphorylation is indeed part of one or both signaling pathways and how and whether Wif1 participates in this process remains to be elucidated in the future.

Secondly, the maintenance of Erk1 phosphorylation in ASZ-Wif1 cells may be related to inhibition of tumor growth. Although, at the first glance this seems to come into conflict with the traditionally regarded role of $\mathrm{pERK} 1 / 2$ as tumor promoters, overexpression of ERK1 in NIH/3T3 cells inhibits Rasmediated proliferation and tumorigenicity while ERK2 does not (Vantaggiato et al. 2006). Furthermore, it has been reported that the cell cycle is promoted when Erk1/2 is persistently and moderately activated, while transient Erk1/2 activation fails to do so (Yamamoto et al. 2006). In addition, sustained Raf/Mek/Erk pathway over-activation can trigger cell cycle arrest (Samuels et al. 1993; Guegan et al. 2014), which can be mediated by elevated expression levels of the cell cycle inhibitor p21cip1 that blocks cyclin E/CDK2 complexes 
inhibiting S-phase entry (LaBaer et al. 1997; Sewing et al. 1997). Finally, temporally elevated Erk1/2 activation at the G2/M transition can block entry into mitosis (Rahmouni et al. 2006). Thus, the consequence of Erk1/2 signaling is considered to depend on the duration, intensity and time point of activation. Accordingly, it is possible that persistent high Erk1 activation in combination with sustained Erk2 activity in ASZ-Wif1 cells may have resulted in cell cycle arrest and thus could ultimately explain the reduced tumor growth of ASZ-Wif1 allografts. This highly interesting topic will be investigated in the future.

In this context it is also interesting to know, that Erk1 and 2 share about $84 \%$ amino acid sequence identity, that they are coexpressed in most tissues, have similar subcellular localization, substrate specificity and stimuli leading to their activation (Samuels et al. 2008; Guihard et al. 2010; Woodson and Kedes 2012). Thus, most investigations did not discriminate between both isoforms. However, and as already mentioned above, increasing evidence supports distinct functions and different outcomes of Erk1 and Erk2 phosphorylation (Pages and Pouyssegur 2004). First hints that they execute different functions came from observations that the levels of Erk1 and Erk2 vary depending on the tissue context (Pages and Pouyssegur 2004). Subsequently, it was shown that loss of ERK1 significantly facilitated proliferation in contrast to knockdown of ERK2 which resulted in reduced proliferation. Vice versa and as already mentioned above, overexpression of ERK1 in NIH/3T3 cells inhibited Ras-mediated proliferation and tumorigenicity while ERK2 does not. Notably, ERK1 function was kinaseindependent in this case, as expression of a catalytically inactive form of ERK1 was equally effective (Vantaggiato et al. 2006). The authors proposed a model in which ERK1 and ERK2 compete in binding to the upstream kinase MEK. By means of this molecular process ERK1 negatively interferes with the growth promoting ERK2 signaling. However, coming back to ASZ-Wif1 cells the total amounts of Erk1 and 2 were not significantly altered but Erk1 phosphorylation was maintained in vivo. Thus, a distinct mechanism may be involved for the tumor-suppressive role of Erk1 in ASZ-Wif1 cells. Indeed, it was hypothesized that Erk1 is a partial agonist of Erk signaling. Thus, Erk1 may bind to the same signaling effectors but is not able to elicit the maximum 
possible response that is produced by the full agonist Erk2. With increasing ratios of Erk1 to Erk2 activity this would result in the inhibition of Erk signaling (Brambilla and Ratto 2009). Thus, increased phosphorylation of Erk1 could possibly inhibit growth promoting effect of Erk2 by competing with its signaling effectors, thereby suppressing ASZ-Wif1 tumor growth in vivo. However, this is pure speculation and remains to be established in the future.

\section{V.2.2.2 Effect of Wif1 on proliferation}

In line with inhibition of proliferation in keratinocytes (Schluter et al. 2013) and with the above-mentioned speculations about the relation of Erk1 and Erk2 activation levels, proliferation and growth of ASZ-Wif1 allografts was suppressed. This was shown by decreased tumor size and weight, which went along with a significantly reduced number of Ki67 positive tumor cells (see Fig. 47). This showed that Wif1 can suppress tumor growth of BCC cells in vivo.

Wif1 has been shown to induce a $\mathrm{G} 1$ cell cycle arrest in invasive bladder cancer cell lines (Tang et al. 2009) and to suppress proliferation in keratinocytes of the interfollicular epidermis (Schluter et al. 2013). Indeed, an in vitro cell cycle analysis showed that ASZ-Wif1 cells had the tendency to accumulate in G1 phase, however this was statistically not significant (see Fig. 21). In addition, the in vitro analysis revealed no significant differences between ASZ-Wif1 and ASZ-pMSCV cells by BrdU assay (see Fig. 22). As Wif1 overexpression did not suppress proliferation in vitro but in vivo we initially hypothesized that the Wif1-mediated inhibition of proliferation in vivo might require a crosstalk with the tumor microenvironment. Within the microenvironment TAMs and CAFs play essential roles in the regulation of tumor cell proliferation (Bingle et al. 2002; Augsten 2014). Therefore, ASZ-Wif1 and ASZ-pMSCV cells were cocultured with several fibroblast cell lines and BMDMs and the proliferative capacity of the BCC cell lines were analyzed. However, no significant difference of the proliferation between both cell lines was observed (see Fig. 23). Thus, the in vivo inhibition of proliferation caused by Wif1 cannot only be explained by an interaction with 
fibroblasts (simulating CAFs) or BMDMs (simulating TAMs) and must rely on additional or different cellular and/or molecular components. Alternatively, and as outlined in the previous section, the Wif1-mediated inhibitory effect on BCC cell proliferation might depend on maintenance of Erk1 phosphorylation in ASZ-Wif1 cells, which may have required cultivation in the absence of FCS. In conclusion, Wif1 inhibits the proliferation of ASZ001 in vivo but not in vitro, at least not in the presence of FCS.

\section{V.2.2.3 Effect of Wif1 on apoptosis}

Since the proliferative capacity of tumor cells is frequently negatively correlated with their metabolic activity and apoptotic processes, WST-1 assay and Annexin V staining were performed. Indeed, WIF1 induces caspase mediated apoptosis in cervical cancer and hepatocellular carcinoma cells (Hu et al. 2009; Ramachandran et al. 2012). Moreover, active Erk1/2 signaling can be associated with both, suppression and induction of apoptosis (Cagnol and Chambard 2010). However, overexpression of Wif1 neither reduced in vitro cell viability as shown by WST-1 assay nor induced cell death as revealed by analysis of Caspase 3 cleavage and Annexin V assay. In accordance, ASZ-Wif1 allografts did not show hints for cell death as determined by TUNEL and p53 antibody staining. Thus, the reduced growth of ASZ-Wif1 allografts did not seem to involve the induction of apoptosis.

\section{V.2.2.4 Effect of Wif1 on differentiation}

As Wif1 expression has been shown to induce differentiation of several cancer entities and also regulates differentiation processes during development (Ramachandran et al. 2014; Baker et al. 2015), I determined whether reduced BCC proliferation was accompanied by an induction of keratinocyte differentiation marker expression. For this reason, the expressions of early markers (loricrin and involucrin) as well as markers for late differentiation (keratin 1 and 10) were analyzed in the ASZ-derived 
allografts. However, no significant differences were detectable. The lack of difference in gene expression together with similar proportions of keratinization in allograft sections (see Fig. 25, 37 and 38) indicates that the anti-proliferative effect mediated by Wif1 is unrelated to induction of differentiation processes.

\section{V.2.2.5 Effect of Wif1 on EMT}

EMT is a prerequisite for the induction of migration that could lead to extravasation and ultimately to metastasis. Thus, migration can serve as a read out for in vitro induction of EMT in epithelial cells (Kalluri and Weinberg 2009). Since Wif1 can induce EMT reversal and can inhibit migration and invasion in prostate cancer cells (Yee et al. 2010), the impact of Wif1 on expression of EMT marker genes and migration was determined.

In vitro, Wif1 did no change the migratory capacity of ASZ001 (see Fig. 24). In accordance with this, the expression of EMT markers Cdh1, Cdh2 and Fn1 did not differ between ASZ-Wif1 and ASZ-pMSCV allografts. This is in line with the microscopically unaffected cell morphology in vitro. Admittedly, we did not expect a significant difference in EMT marker expression or migration as ASZ001 already show a strict epithelial phenotype. In addition, these cells are derived from a well differentiated semi-malignant tumor entity, which is maintained when the cells were transplanted in mice and formed tumors (see Fig. 28). Taken together, we conclude that Wif1 probably does not affect EMT in BCC. Furthermore, Wif1 seems to be unable to induce a more epithelial phenotype of an already well differentiated epithelial tumor.

\section{V.2.2.6 Effect of Wif1 on the tumor microenvironment}

As discussed in section IV.5.2.6 antibody staining of the S-phase marker Ki67 showed reduced proliferation of ASZ-Wif1 derived tumors. Since invasion of TAMs and CAFs can affect tumor growth, antibody stainings 
using an anti-F4/80 antibody and an anti-aSma antibody, respectively, were conducted.

TAMs and CAFs can either promote or inhibit tumor growth and the respective outcome is apparently context-specific (Bingle et al. 2002; Augsten 2014). TAMs and CAFs secrete a variety of growth factor, including EGF, hepatocyte growth factor (HGF), basic fibroblastic growth factor (bFGF), vascular endothelial growth factor (VEGF) and platelet derived growth factor (PDGF) (Koontongkaew 2013) that can modulate tumor growth. Thus, the recruitment or absence of TAMs and/or CAFs could have been a cause of the growth inhibitory effect of Wif1.

However, no obvious differences in the number of $F 4 / 80$ and aSma positive cells were detected in ASZ-Wif1 and ASZ-pMSCV derived tumors when stained with the respective antibodies (see Fig. 49 and 50). Admittedly, this does not categorically exclude differences in e.g. activity or secretion of growth factors of TAMs or CAFs. However, the similar infiltration pattern of the tumor samples together with the fact that the corresponding coculture experiments also did not result in proliferation changes of the BCC cells (see section IV.4.3 and Fig. 23) kept us from further extensions of the experiments.

Furthermore, extracellular matrix (ECM) proteins including collagens can significantly influence tumor growth and invasiveness. This is due to the fact that tumor progression directly depends on the ECM composition, structure and organization (Yu et al. 2011). Traditionally, ECM deposition is considered as an antagonistic response of the host stroma to the development of the tumor.

Undoubtedly, tumor cells change their microenvironment to ensure their survival (Bissell and Radisky 2001). Furthermore, previous data showed that inhibition of integrins that mediate cell-ECM interactions results in restoration of a "close-to-benign" phenotype of tumor cells (Weaver et al. 1997). Moreover, it is well known that tumor-derived ECM is biochemically distinct in its composition compared to normal ECM. This is responsible for a stiffening of the tumor stroma compared to normal stroma (Kass et al. 2007; Levental et al. 2009). Thus, ECM deposition has been linked to tumor growth. 
However, in contrast it also has been reported that overexpression of type I collagen, which is a main component of the ECM, can reduce tumor growth in vivo (Honma et al. 2007).

Due to the importance of ECM in tumor growth processes, the collagen deposition was analyzed in ASZ-derived allograft sections by means of Ladewig staining. Collagen deposition was significantly increased in deeper necrotic areas of ASZ-Wif1 allografts when compared to ASZ-pMSCV allografts. Outer regions of the allografts with mainly vital tumor tissue showed a tendency of elevated collagen levels that, however, was not significant. Nevertheless, it would be certainly of interest to study the effects of Wif1 on production of collagen and thus on ECM in more detail. In addition, it would be also interesting to analyze whether Wif1 changes the composition of the ECM or modulates the expression of e.g. metalloproteases that are well known ECM degrading enzymes.

Admittedly, it remains to be said that the increased amount of collagen found in the necrotic tumor regions may have originated from the Matrigel that served as substrate for the cells transplanted cells into nude mice.

Nevertheless, the collagen accumulation in ASZ-Wif1 allografts is an interesting feature that certainly deserves further investigations.

\section{V.3 Wif1 depletion in murine BCC}

The tumor suppressive role of Wif1 in BCC was verified in a genetic approach. Wif1 disruption led to tumor promotion, which supported the antitumorigenic role of Wif1 in BCC. In this genetic approach the Ptch $^{\text {flox/flox }}$ CreERT2 ${ }^{T /-}$ BCC mouse model was bred onto a Wif1 deficient background. The resulting Ptch ${ }^{f l o x / f l o x} C r e E R T 2^{T /-} W_{i f 1^{-/}}$offspring lacked Wif1 expression in BCC as estimated by Western blot. Ptch ${ }^{\text {flox/flox }}$ CreERT2 ${ }^{T /-}$ and Ptch ${ }^{\text {flox } / \text { llox }}$ Wif $1^{-/-}$littermates served as controls. For each genotype 2 cohorts were sacrificed $45 \mathrm{~d}$ and $90 \mathrm{~d}$ after tamoxifen injection. Macroscopically, first tumors were visible about 4 weeks after tamoxifen injection in

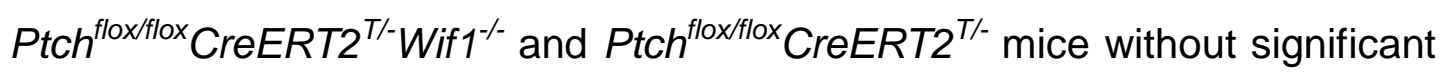
differences between the genotypes. As expected Cre lacking Ptch ${ }^{\text {flox/flox }}$ Wif $^{-1-}$ 
mice remained tumor free. In the cohorts that were sacrificed $45 \mathrm{~d}$ after

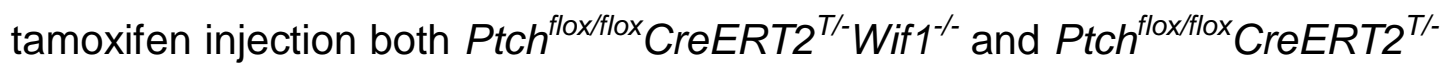
mice developed histologically confirmed BCC. At that time the tumor load of the animals was undistinguishable. Taken together with lacking differences in tumor onset, this suggests that Wif1 does not affect tumor initiation. However, when the animals were sacrificed 90d after tumor induction the tumor load of Ptch ${ }^{\text {flox } f l o x}$ CreERT2 ${ }^{T /}$ Wif $1^{-/}$was significantly increased when compared to Ptch ${ }^{\text {floxflox }}$ CreERT2 ${ }^{T /}$ mice. In addition, we confirmed the enhanced proliferative capacity of the tumors as the number of Ki67 positive cells was significantly higher in tumors lacking Wif1. Together, this experiment confirmed that Wif1 plays a tumor suppressive role in BCC progression.

\section{V.4 Outlook}

Planimetric measurement and Ki67 staining show that BCC growth is enhanced in Ptch knockout mice on a Wif1-deficient background. Vice versa, tumor-intrinsic Wif1 overexpression in the BCC cell line ASZ001 inhibits tumor growth in mice. This does not involve apoptosis, Akt, Wnt or Hh signaling or activation of apoptosis. It also does not involve changes in tumor differentiation or alterations in stromal composition of transplanted cells in host mice. Furthermore it does not seem to require an interaction with tumor associated fibroblasts or macrophages. Because tumor-intrinsic Wif1 expression only inhibits cellular proliferation in vivo but not in vitro the data suggest that Wif1 probably prevents tumor-promoting effects of soluble Wnt or other factors secreted by the tumor environment.

The tumor suppressive effect of Wif1 was exclusively observed in vivo. Thus, Wif1 could elicit its effects via two potential mechanisms. First, secreted Wif1 may result in the activation of $\mathrm{Wnt} / \mathrm{Ca}^{2+}$ or affect EGFR signaling and ultimately lead to PKC and Erk1 phosphorylation of the secreting cells in an autocrine fashion. Second, secreted Wif1 may also affect cells of the tumor stroma in a paracrine fashion. Mediated by the inhibition of Wnt signaling or by affecting EGFR signaling in adjacent stromal cells, Wif1 might change the 
secretome of these cells, which in turn could result in the outcome described above (see Fig. 56). In order to elucidate whether paracrine signaling mechanisms are participating in the observed molecular events one could perform expression analyses using antibody staining or Western blot detecting putatively involved signaling molecules comparing ASZ-Wif1 and ASZ-pMSCV allografts.

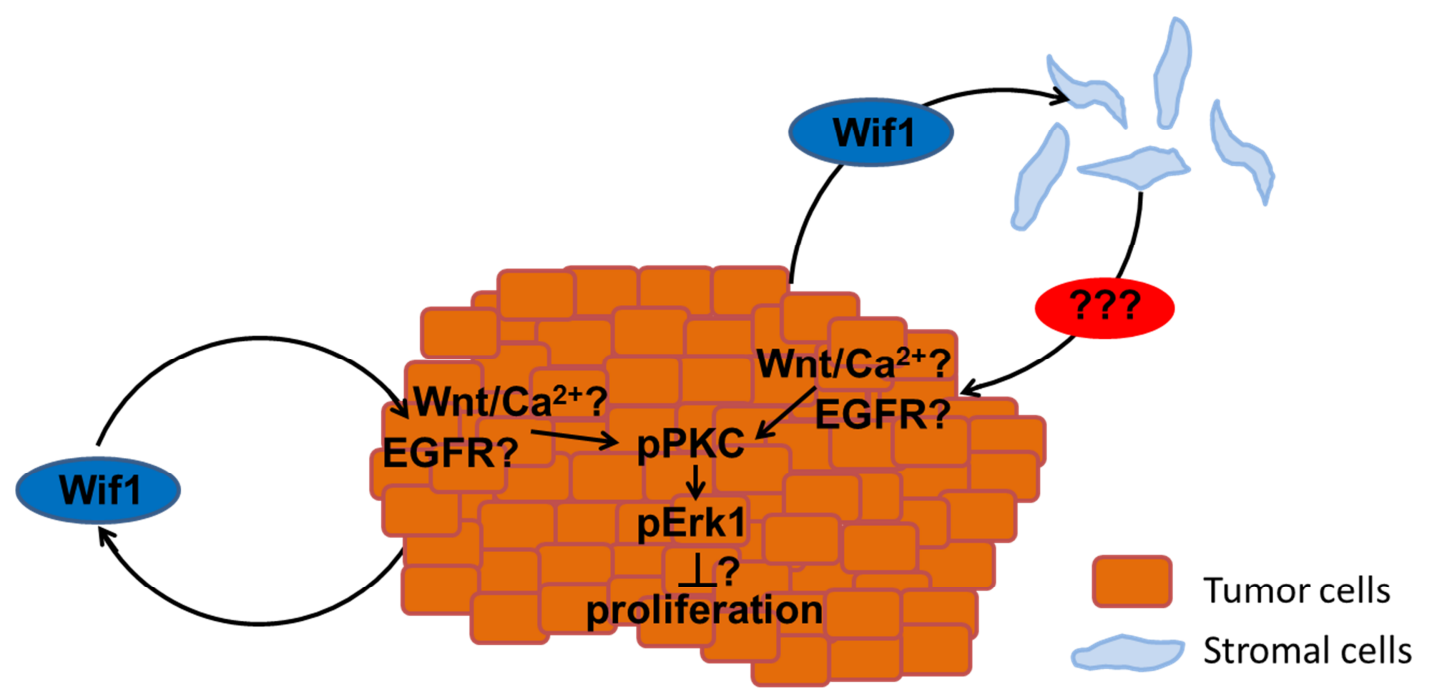

Fig. 56: Putative autocrine and paracrine signaling mechanism in ASZ-Wif1 allografts

To examine the causative mechanism underlying these observations one should firstly analyse the proliferative capacity of ASZ-Wif1 in the absence of FCS, because the surplus of growth factors in FCS may have impeded the Wif1-dependent inhibition of proliferation in vitro. Furthermore, in the same experimental setting an incubation of ASZ-pMSCV with concentrated ASZ-Wif1 supernatant would confirm whether Wif1 inhibits proliferation.

Most interestingly, the Wif1-mediated prevention of in vivo tumor growth was accompanied by maintenance of phosphorylation of PKC and Erk1 that was already seen in cell cultures, whereas phosphorylation of both proteins vanished in the faster growing control cells. This indicates that Wif1 may permit Wnt/Ca ${ }^{2+}$ signaling mediated by PKC phosphorylation resulting in Erk1 activation. Alternatively, Wif1 might also affect EGFR signaling. Nevertheless, specific inhibition of Erk1 would demonstrate whether Erk1 phosphorylation could result in the suppression of proliferation. Unfortunately, specific Erk1 inhibitors are not available. However, inhibition of Mek1/2 by U0126 resulting in inhibition of Erk1/2 was shown to inhibit proliferation of 
ASZ001 (Xie et al. 2001). Therefore, one should also anticipate an Erk1 knockdown/-out experiments in ASZ-Wif1 cells. This experiment would help to elucidate the function of Erk1 in proliferation in vitro and tumor growth suppression in an allograft experiment.

To investigate whether EGFR signaling participates in Erk1 activation, protein samples from the respective in vitro cultures as well as allograft samples should be analyzed regarding EGFR signaling activity. First, the phosphorylation status of EGF receptors ErbB2, ErbB3, and ErbB4 that are able to bind Wif1 should be determined. Downstream of ErbB, receptor components of the signaling pathway that are known to be regulators of Erk1 activity (e.g. Ras, Raf, Mek1) and effector proteins that are known to be regulated by Erk1 and control proliferation should be analyzed regarding their activity in subsequent experiments.

In summary, our data indicate that Wif1 exerts a tumor suppressive function in BCC. Therefore, it might be worth testing purified and concentrated, topically applied Wif1 in a therapeutic treatment approach in the Ptch mouse model for BCC. 


\section{References}

Abdulla, FR, Feldman, SR, Williford, PM, Krowchuk, D and Kaur, M (2005). "Tanning and skin cancer." Pediatr Dermatol 22(6): 501-512.

Arteaga, CL and Engelman, JA (2014). "ERBB receptors: from oncogene discovery to basic science to mechanism-based cancer therapeutics." Cancer Cell 25(3): 282-303.

Athar, M, Tang, X, Lee, JL, Kopelovich, L and Kim, AL (2006). "Hedgehog signalling in skin development and cancer." Exp Dermatol 15(9): 667677.

Augsten, M (2014). "Cancer-associated fibroblasts as another polarized cell type of the tumor microenvironment." Front Oncol 4: 62.

Augustin, I, Gross, J, Baumann, D, Korn, C, Kerr, G, Grigoryan, T, Mauch, C, Birchmeier, W and Boutros, M (2013). "Loss of epidermal Evi/Wls results in a phenotype resembling psoriasiform dermatitis." J Exp Med 210(9): 1761-1777.

Avanesov, A and Blair, SS (2013). "The Drosophila WIF1 homolog Shifted maintains glypican-independent Hedgehog signaling and interacts with the Hedgehog co-receptors Ihog and Boi." Development 140(1): 107116.

Avanesov, A, Honeyager, SM, Malicki, J and Blair, SS (2012). "The role of glypicans in Wnt inhibitory factor-1 activity and the structural basis of Wif1's effects on Wnt and Hedgehog signaling." PLoS Genet 8(2): e1002503.

Baker, EK, Taylor, S, Gupte, A, Chalk, AM, Bhattacharya, S, Green, AC, Martin, TJ, Strbenac, D, Robinson, MD, Purton, LE and Walkley, CR (2015). "Wnt inhibitory factor 1 (WIF1) is a marker of osteoblastic differentiation stage and is not silenced by DNA methylation in osteosarcoma." Bone 73: 223-232.

Banziger, C, Soldini, D, Schutt, C, Zipperlen, P, Hausmann, G and Basler, K (2006). "Wntless, a conserved membrane protein dedicated to the secretion of Wnt proteins from signaling cells." Cell 125(3): 509-522.

Basset-Seguin, N, Sharpe, HJ and de Sauvage, FJ (2015). "Efficacy of Hedgehog Pathway Inhibitors in Basal Cell Carcinoma." Mol Cancer Ther.

Beachy, PA, Hymowitz, SG, Lazarus, RA, Leahy, DJ and Siebold, C (2010). "Interactions between Hedgehog proteins and their binding partners come into view." Genes Dev 24(18): 2001-2012.

Beachy, PA, Karhadkar, SS and Berman, DM (2004). "Tissue repair and stem cell renewal in carcinogenesis." Nature 432(7015): 324-331.

Becker, M (2011). "The Role of Wif1 in Basal Cell Carcinoma" Master Thesis

Behrens, J, von Kries, JP, Kuhl, M, Bruhn, L, Wedlich, D, Grosschedl, R and Birchmeier, W (1996). "Functional interaction of beta-catenin with the transcription factor LEF-1." Nature 382(6592): 638-642. 
Bingle, L, Brown, NJ and Lewis, CE (2002). "The role of tumour-associated macrophages in tumour progression: implications for new anticancer therapies." J Pathol 196(3): 254-265.

Bissell, MJ and Radisky, D (2001). "Putting tumours in context." Nat Rev Cancer 1(1): 46-54.

Boonchai, W, Green, A, Ng, J, Dicker, A and Chenevix-Trench, G (2000). "Basal cell carcinoma in chronic arsenicism occurring in Queensland, Australia, after ingestion of an asthma medication." J Am Acad Dermatol 43(4): 664-669.

Bowman, T, Garcia, R, Turkson, J and Jove, R (2000). "STATs in oncogenesis." Oncogene 19(21): 2474-2488.

Brambilla, R and Ratto, GM (2009). "The controversy on the differential role of ERK1 and ERK2 MAP kinases in cell signaling" Letter to the editor of Cell Cycle

Breitkreutz, D, Braiman-Wiksman, L, Daum, N, Denning, MF and Tennenbaum, T (2007). "Protein kinase $C$ family: on the crossroads of cell signaling in skin and tumor epithelium." J Cancer Res Clin Oncol 133(11): 793-808.

Briscoe, J, Chen, Y, Jessell, TM and Struhl, G (2001). "A hedgehoginsensitive form of patched provides evidence for direct long-range morphogen activity of sonic hedgehog in the neural tube." Mol Cell 7(6): 1279-1291.

Burke, R, Nellen, D, Bellotto, M, Hafen, E, Senti, KA, Dickson, BJ and Basler, K (1999). "Dispatched, a novel sterol-sensing domain protein dedicated to the release of cholesterol-modified hedgehog from signaling cells." Cell 99(7): 803-815.

Cadigan, KM and Waterman, ML (2012). "TCF/LEFs and Wnt signaling in the nucleus." Cold Spring Harb Perspect Biol 4(11).

Cagnol, S and Chambard, JC (2010). "ERK and cell death: mechanisms of ERK-induced cell death--apoptosis, autophagy and senescence." FEBS J 277(1): 2-21.

Cancer Facts \& Figures (2015). Atlanta: American Cancer Society

Carstens, PO (2010). "Untersuchungen zur Rolle von Wnt5a Beim Basalzellkarzinom" Dissertation

Cebrat, M, Strzadala, L and Kisielow, P (2004). "Wnt inhibitory factor-1: a candidate for a new player in tumorigenesis of intestinal epithelial cells." Cancer Lett 206(1): 107-113.

Chang, SC, Mulloy, B, Magee, Al and Couchman, JR (2011). "Two distinct sites in sonic Hedgehog combine for heparan sulfate interactions and cell signaling functions." J Biol Chem 286(52): 44391-44402.

Chong, CR and Janne, PA (2013). "The quest to overcome resistance to EGFR-targeted therapies in cancer." Nat Med 19(11): 1389-1400.

Clevers, $\mathrm{H}$ and Nusse, R (2012). "Wnt/beta-catenin signaling and disease." Cell 149(6): 1192-1205.

Crowson, AN (2006). "Basal cell carcinoma: biology, morphology and clinical implications." Mod Pathol 19 Suppl 2: S127-147. 
Curson, C and Weedon, D (1979). "Spontaneous regression in basal cell carcinomas." J Cutan Pathol 6(5): 432-437.

Dessinioti, C, Antoniou, C, Katsambas, A and Stratigos, AJ (2010). "Basal cell carcinoma: what's new under the sun." Photochem Photobiol 86(3): 481-491.

Devenport, D (2014). "The cell biology of planar cell polarity." J Cell Biol 207(2): 171-179.

Doglioni, C, Piccinin, S, Demontis, S, Cangi, MG, Pecciarini, L, Chiarelli, C, Armellin, M, Vukosavljevic, T, Boiocchi, M and Maestro, R (2003). "Alterations of beta-catenin pathway in non-melanoma skin tumors: loss of alpha-ABC nuclear reactivity correlates with the presence of beta-catenin gene mutation." Am J Pathol 163(6): 2277-2287.

Eberl, M, Klingler, S, Mangelberger, D, Loipetzberger, A, Damhofer, H, Zoidl, K, Schnidar, H, Hache, H, Bauer, HC, Solca, F, Hauser-Kronberger, C, Ermilov, AN, Verhaegen, ME, Bichakjian, CK, Dlugosz, AA, Nietfeld, W, Sibilia, M, Lehrach, H, Wierling, C and Aberger, F (2012). "Hedgehog-EGFR cooperation response genes determine the oncogenic phenotype of basal cell carcinoma and tumour-initiating pancreatic cancer cells." EMBO Mol Med 4(3): 218-233.

Ecke, I (2008). "Überprüfung neuer Therapieansätze für Patched-assoziierte Tumoren im Mausmodell" Dissertation

El-Bahrawy, M, El-Masry, N, Alison, M, Poulsom, R and Fallowfield, M (2003). "Expression of beta-catenin in basal cell carcinoma." $\mathrm{Br} \mathrm{J}$ Dermatol 148(5): 964-970.

Epstein, EH (2008). "Basal cell carcinomas: attack of the hedgehog." Nat Rev Cancer 8(10): 743-754.

Euhus, DM, Hudd, C, LaRegina, MC and Johnson, FE (1986). "Tumor measurement in the nude mouse." J Surg Oncol 31(4): 229-234.

Fan, QW, Cheng, C, Knight, ZA, Haas-Kogan, D, Stokoe, D, James, CD, McCormick, F, Shokat, KM and Weiss, WA (2009). "EGFR signals to mTOR through PKC and independently of Akt in glioma." Sci Signal 2(55): ra4.

Fullar, A, Dudas, J, Olah, L, Hollosi, P, Papp, Z, Sobel, G, Karaszi, K, Paku, S, Baghy, $\mathrm{K}$ and Kovalszky, I (2015). "Remodeling of extracellular matrix by normal and tumor-associated fibroblasts promotes cervical cancer progression." BMC Cancer 15: 256.

Glise, B, Miller, CA, Crozatier, M, Halbisen, MA, Wise, S, Olson, DJ, Vincent, $A$ and Blair, SS (2005). "Shifted, the Drosophila ortholog of Wnt inhibitory factor-1, controls the distribution and movement of Hedgehog." Dev Cell 8(2): 255-266.

Goppner, D and Leverkus, M (2011). "Basal cell carcinoma: from the molecular understanding of the pathogenesis to targeted therapy of progressive disease." J Skin Cancer 2011: 650258.

Gorfinkiel, N, Sierra, J, Callejo, A, Ibanez, C and Guerrero, I (2005). "The Drosophila ortholog of the human Wnt inhibitor factor Shifted controls the diffusion of lipid-modified Hedgehog." Dev Cell 8(2): 241-253. 
Green, J, Nusse, R and van Amerongen, R (2014). "The role of Ryk and Ror receptor tyrosine kinases in Wnt signal transduction." Cold Spring Harb Perspect Biol 6(2).

Guegan, JP, Ezan, F, Gailhouste, L, Langouet, S and Baffet, G (2014). "MEK1/2 overactivation can promote growth arrest by mediating ERK1/2-dependent phosphorylation of p70S6K." J Cell Physiol 229(7): 903-915.

Guihard, S, Clay, D, Cocault, L, Saulnier, N, Opolon, P, Souyri, M, Pages, G, Pouyssegur, J, Porteu, F and Gaudry, M (2010). "The MAPK ERK1 is a negative regulator of the adult steady-state splenic erythropoiesis." Blood 115(18): 3686-3694.

Habas, R, Dawid, IB and He, X (2003). "Coactivation of Rac and Rho by Wnt/Frizzled signaling is required for vertebrate gastrulation." Genes Dev 17(2): 295-309.

Hafner, C, Landthaler, M and Vogt, T (2010). "Activation of the PI3K/AKT signalling pathway in non-melanoma skin cancer is not mediated by oncogenic PIK3CA and AKT1 hotspot mutations." Exp Dermatol 19(8): e222-227.

Hahn, H, Wicking, C, Zaphiropoulous, PG, Gailani, MR, Shanley, S, Chidambaram, A, Vorechovsky, I, Holmberg, E, Unden, AB, Gillies, S, Negus, K, Smyth, I, Pressman, C, Leffell, DJ, Gerrard, B, Goldstein, AM, Dean, M, Toftgard, R, Chenevix-Trench, G, Wainwright, B and Bale, AE (1996). "Mutations of the human homolog of Drosophila patched in the nevoid basal cell carcinoma syndrome." Cell 85(6): 841-851.

Hallikas, O, Palin, K, Sinjushina, N, Rautiainen, R, Partanen, J, Ukkonen, E and Taipale, $J$ (2006). "Genome-wide prediction of mammalian enhancers based on analysis of transcription-factor binding affinity." Cell 124(1): 47-59.

Haqq, C, Nosrati, M, Sudilovsky, D, Crothers, J, Khodabakhsh, D, Pulliam, BL, Federman, S, Miller, JR, 3rd, Allen, RE, Singer, MI, Leong, SP, Ljung, BM, Sagebiel, RW and Kashani-Sabet, M (2005). "The gene expression signatures of melanoma progression." Proc Natl Acad Sci U S A 102(17): 6092-6097.

Hemmings, BA and Restuccia, DF (2015). "The PI3K-PKB/Akt pathway." Cold Spring Harb Perspect Biol 7(4).

Hogan, PG, Chen, L, Nardone, J and Rao, A (2003). "Transcriptional regulation by calcium, calcineurin, and NFAT." Genes Dev 17(18): 2205-2232.

Honma, K, Miyata, T and Ochiya, T (2007). "Type I collagen gene suppresses tumor growth and invasion of malignant human glioma cells." Cancer Cell Int 7: 12.

Hsieh, JC, Kodjabachian, L, Rebbert, ML, Rattner, A, Smallwood, PM, Samos, CH, Nusse, R, Dawid, IB and Nathans, J (1999). "A new secreted protein that binds to Wnt proteins and inhibits their activities." Nature 398(6726): 431-436. 
Hu, J, Dong, A, Fernandez-Ruiz, V, Shan, J, Kawa, M, Martinez-Anso, E, Prieto, J and Qian, C (2009). "Blockade of Wnt signaling inhibits angiogenesis and tumor growth in hepatocellular carcinoma." Cancer Res 69(17): 6951-6959.

Huang, S, Zhong, X, Gao, J, Song, R, Wu, H, Zi, S, Yang, S, Du, P, Cui, L, Yang, C and Li, Z (2014). "Coexpression of SFRP1 and WIF1 as a prognostic predictor of favorable outcomes in patients with colorectal carcinoma." Biomed Res Int 2014: 256723.

Hui, CC and Angers, S (2011). "Gli proteins in development and disease." Annu Rev Cell Dev Biol 27: 513-537.

Johnson, RL, Rothman, AL, Xie, J, Goodrich, LV, Bare, JW, Bonifas, JM, Quinn, AG, Myers, RM, Cox, DR, Epstein, EH, Jr. and Scott, MP (1996). "Human homolog of patched, a candidate gene for the basal cell nevus syndrome." Science 272(5268): 1668-1671.

Kalluri, R and Weinberg, RA (2009). "The basics of epithelial-mesenchymal transition." J Clin Invest 119(6): 1420-1428.

Kansara, M, Tsang, M, Kodjabachian, L, Sims, NA, Trivett, MK, Ehrich, M, Dobrovic, A, Slavin, J, Choong, PF, Simmons, PJ, Dawid, IB and Thomas, DM (2009). "Wnt inhibitory factor 1 is epigenetically silenced in human osteosarcoma, and targeted disruption accelerates osteosarcomagenesis in mice." J Clin Invest 119(4): 837-851.

Kass, L, Erler, JT, Dembo, M and Weaver, VM (2007). "Mammary epithelial cell: influence of extracellular matrix composition and organization during development and tumorigenesis." Int J Biochem Cell Biol 39(11): 1987-1994.

Kaucka, M, Petersen, J, Janovska, P, Radaszkiewicz, T, Smyckova, L, Daulat, AM, Borg, JP, Schulte, G and Bryja, V (2015). "Asymmetry of VANGL2 in migrating lymphocytes as a tool to monitor activity of the mammalian WNT/planar cell polarity pathway." Cell Commun Signal 13: 2.

Kawakami, K, Hirata, H, Yamamura, S, Kikuno, N, Saini, S, Majid, S, Tanaka, Y, Kawamoto, K, Enokida, H, Nakagawa, M and Dahiya, R (2009). "Functional significance of Wnt inhibitory factor-1 gene in kidney cancer." Cancer Res 69(22): 8603-8610.

Kim, GH and Han, JK (2005). "JNK and ROKalpha function in the noncanonical Wnt/RhoA signaling pathway to regulate Xenopus convergent extension movements." Dev Dyn 232(4): 958-968.

Kim, J, You, L, Xu, Z, Kuchenbecker, K, Raz, D, He, B and Jablons, D (2007). "Wnt inhibitory factor inhibits lung cancer cell growth." J Thorac Cardiovasc Surg 133(3): 733-737.

Kim, JY, Lee, TR and Lee, AY (2013). "Reduced WIF-1 expression stimulates skin hyperpigmentation in patients with melasma." J Invest Dermatol 133(1): 191-200.

Kispert, A, Vainio, S and McMahon, AP (1998). "Wnt-4 is a mesenchymal signal for epithelial transformation of metanephric mesenchyme in the developing kidney." Development 125(21): 4225-4234. 
Kolch, W (2005). "Coordinating ERK/MAPK signalling through scaffolds and inhibitors." Nat Rev Mol Cell Biol 6(11): 827-837.

König, S (2012). "Die Rolle vonWnt5a bei der Regression des Basalzellkarzinoms" Dissertation

Koontongkaew, S (2013). "The tumor microenvironment contribution to development, growth, invasion and metastasis of head and neck squamous cell carcinomas." J Cancer 4(1): 66-83.

Korinek, V, Barker, N, Morin, PJ, van Wichen, D, de Weger, R, Kinzler, KW, Vogelstein, B and Clevers, H (1997). "Constitutive transcriptional activation by a beta-catenin-Tcf complex in APC-/- colon carcinoma." Science 275(5307): 1784-1787.

Krahn, G, Leiter, U, Kaskel, P, Udart, M, Utikal, J, Bezold, G and Peter, RU (2001). "Coexpression patterns of EGFR, HER2, HER3 and HER4 in non-melanoma skin cancer." Eur J Cancer 37(2): 251-259.

Kuhl, M, Sheldahl, LC, Malbon, CC and Moon, RT (2000). "Ca(2+)/calmodulin-dependent protein kinase II is stimulated by Wnt and Frizzled homologs and promotes ventral cell fates in Xenopus." J Biol Chem 275(17): 12701-12711.

Kulesz-Martin, M, Kilkenny, AE, Holbrook, KA, Digernes, V and Yuspa, SH (1983). "Properties of carcinogen altered mouse epidermal cells resistant to calcium-induced terminal differentiation." Carcinogenesis 4(11): 1367-1377.

LaBaer, J, Garrett, MD, Stevenson, LF, Slingerland, JM, Sandhu, C, Chou, HS, Fattaey, A and Harlow, E (1997). "New functional activities for the p21 family of CDK inhibitors." Genes Dev 11(7): 847-862.

Lam, CW, Xie, J, To, KF, Ng, HK, Lee, KC, Yuen, NW, Lim, PL, Chan, LY, Tong, SF and McCormick, F (1999). "A frequent activated smoothened mutation in sporadic basal cell carcinomas." Oncogene 18(3): 833836.

Levental, KR, Yu, H, Kass, L, Lakins, JN, Egeblad, M, Erler, JT, Fong, SF, Csiszar, K, Giaccia, A, Weninger, W, Yamauchi, M, Gasser, DL and Weaver, VM (2009). "Matrix crosslinking forces tumor progression by enhancing integrin signaling." Cell 139(5): 891-906.

Li, VS, Ng, SS, Boersema, PJ, Low, TY, Karthaus, WR, Gerlach, JP, Mohammed, S, Heck, AJ, Maurice, MM, Mahmoudi, T and Clevers, H (2012). "Wnt signaling through inhibition of beta-catenin degradation in an intact Axin1 complex." Cell 149(6): 1245-1256.

Li, XJ, Zhang, X, Johnson, MA, Wang, ZB, Lavaute, T and Zhang, SC (2009). "Coordination of sonic hedgehog and Wnt signaling determines ventral and dorsal telencephalic neuron types from human embryonic stem cells." Development 136(23): 4055-4063.

Lim, X and Nusse, R (2013). "Wnt signaling in skin development, homeostasis, and disease." Cold Spring Harb Perspect Biol 5(2).

Lin, YC, You, L, Xu, Z, He, B, Yang, CT, Chen, JK, Mikami, I, Clement, G, Shi, Y, Kuchenbecker, K, Okamoto, J, Kashani-Sabet, M and Jablons, DM (2007). "Wnt inhibitory factor-1 gene transfer inhibits melanoma cell growth." Hum Gene Ther 18(4): 379-386. 
Liu, F, van den Broek, O, Destree, O and Hoppler, S (2005). "Distinct roles for Xenopus Tcf/Lef genes in mediating specific responses to Wnt/beta-catenin signalling in mesoderm development." Development 132(24): 5375-5385.

Lories, RJ, Corr, M and Lane, NE (2013). "To Wnt or not to Wnt: the bone and joint health dilemma." Nat Rev Rheumatol 9(6): 328-339.

Louro, ID, Bailey, EC, Li, X, South, LS, McKie-Bell, PR, Yoder, BK, Huang, CC, Johnson, MR, Hill, AE, Johnson, RL and Ruppert, JM (2002). "Comparative gene expression profile analysis of GLI and C-MYC in an epithelial model of malignant transformation." Cancer Res 62(20): 5867-5873.

Lustig, B, Jerchow, B, Sachs, M, Weiler, S, Pietsch, T, Karsten, U, van de Wetering, M, Clevers, H, Schlag, PM, Birchmeier, W and Behrens, J (2002). "Negative feedback loop of Wnt signaling through upregulation of conductin/axin2 in colorectal and liver tumors." Mol Cell Biol 22(4): 1184-1193.

Ma, Y, Erkner, A, Gong, R, Yao, S, Taipale, J, Basler, K and Beachy, PA (2002). "Hedgehog-mediated patterning of the mammalian embryo requires transporter-like function of dispatched." Cell 111(1): 63-75.

Malinauskas, T, Aricescu, AR, Lu, W, Siebold, C and Jones, EY (2011). "Modular mechanism of Wnt signaling inhibition by Wnt inhibitory factor 1." Nat Struct Mol Biol 18(8): 886-893.

Malinauskas, T and Jones, EY (2014). "Extracellular modulators of Wnt signalling." Curr Opin Struct Biol 29: 77-84.

Mancuso, M, Pazzaglia, S, Tanori, M, Hahn, H, Merola, P, Rebessi, S, Atkinson, MJ, Di Majo, V, Covelli, V and Saran, A (2004). "Basal cell carcinoma and its development: insights from radiation-induced tumors in Ptch1-deficient mice." Cancer Res 64(3): 934-941.

Mazieres, J, He, B, You, L, Xu, Z, Lee, AY, Mikami, I, Reguart, N, Rosell, R, McCormick, F and Jablons, DM (2004). "Wnt inhibitory factor-1 is silenced by promoter hypermethylation in human lung cancer." Cancer Res 64(14): 4717-4720.

McCubrey, JA, Steelman, LS, Chappell, WH, Abrams, SL, Franklin, RA, Montalto, G, Cervello, M, Libra, M, Candido, S, Malaponte, G, Mazzarino, MC, Fagone, P, Nicoletti, F, Basecke, J, Mijatovic, S, Maksimovic-Ivanic, D, Milella, M, Tafuri, A, Chiarini, F, Evangelisti, C, Cocco, L and Martelli, AM (2012). "Ras/Raf/MEK/ERK and $\mathrm{PI3K} / \mathrm{PTEN} / \mathrm{Akt} / \mathrm{mTOR}$ cascade inhibitors: how mutations can result in therapy resistance and how to overcome resistance." Oncotarget 3(10): 1068-1111.

Mendoza, MC, Er, EE and Blenis, J (2011). "The Ras-ERK and PI3K-mTOR pathways: cross-talk and compensation." Trends Biochem Sci 36(6): 320-328.

Molenaar, $M$, van de Wetering, $M$, Oosterwegel, $M$, Peterson-Maduro, J, Godsave, S, Korinek, V, Roose, J, Destree, O and Clevers, H (1996). "XTcf-3 transcription factor mediates beta-catenin-induced axis formation in Xenopus embryos." Cell 86(3): 391-399. 
Morin, PJ, Sparks, AB, Korinek, V, Barker, N, Clevers, H, Vogelstein, B and Kinzler, KW (1997). "Activation of beta-catenin-Tcf signaling in colon cancer by mutations in beta-catenin or APC." Science 275(5307): 1787-1790.

Nakayama, M, Tabuchi, K, Nakamura, Y and Hara, A (2011). "Basal cell carcinoma of the head and neck." J Skin Cancer 2011: 496910.

Neill, GW, Ghali, LR, Green, JL, Ikram, MS, Philpott, MP and Quinn, AG (2003). "Loss of protein kinase Calpha expression may enhance the tumorigenic potential of Gli1 in basal cell carcinoma." Cancer Res 63(15): 4692-4697.

Nilsson, M, Unden, AB, Krause, D, Malmqwist, U, Raza, K, Zaphiropoulos, PG and Toftgard, R (2000). "Induction of basal cell carcinomas and trichoepitheliomas in mice overexpressing GLI-1." Proc Natl Acad Sci U S A 97(7): 3438-3443.

Nitzki, F (2008). "Patched-assoziierte Tumoren: Modifikatorgene und Pathogenese" Dissertation

Nitzki, F, Zibat, A, König, S, Wijgerde, M, Rosenberger, A, Brembeck, FH, Carstens, P-O, Frommhold, A, Uhmann, A, Klingler, S, Reifenberger, J, Pukrop, T, Aberger, F, Schulz-Schaeffer, W and Hahn, H (2010). "Tumor stroma-derived Wnt5a induces differentiation of basal cell carcinoma of Ptch mutant mice via CaMKII." Cancer Res in press.

Noubissi, FK, Kim, T, Kawahara, TN, Aughenbaugh, WD, Berg, E, Longley, BJ, Athar, M and Spiegelman, VS (2014). "Role of CRD-BP in the growth of human basal cell carcinoma cells." J Invest Dermatol 134(6): 1718-1724.

Nyati, MK, Morgan, MA, Feng, FY and Lawrence, TS (2006). "Integration of EGFR inhibitors with radiochemotherapy." Nat Rev Cancer 6(11): 876885.

Oliva, JL, Griner, EM and Kazanietz, MG (2005). "PKC isozymes and diacylglycerol-regulated proteins as effectors of growth factor receptors." Growth Factors 23(4): 245-252.

Oro, AE, Higgins, KM, Hu, Z, Bonifas, JM, Epstein, EH, Jr. and Scott, MP (1997). "Basal cell carcinomas in mice overexpressing sonic hedgehog." Science 276(5313): 817-821.

Pages, G and Pouyssegur, J (2004). "Study of MAPK signaling using knockout mice." Methods Mol Biol 250: 155-166.

Palmer, HG, Anjos-Afonso, F, Carmeliet, G, Takeda, H and Watt, FM (2008). "The vitamin $D$ receptor is a Wnt effector that controls hair follicle differentiation and specifies tumor type in adult epidermis." PLoS One 3(1): e1483.

Park, TJ, Kim, M, Kim, H, Park, SY, Park, KC, Ortonne, JP and Kang, HY (2014). "Wnt inhibitory factor (WIF)-1 promotes melanogenesis in normal human melanocytes." Pigment Cell Melanoma Res 27(1): 7281.

Pasca di Magliano, M and Hebrok, M (2003). "Hedgehog signalling in cancer formation and maintenance." Nat Rev Cancer 3(12): 903-911.

Patthy, L (2000). "The WIF module." Trends Biochem Sci 25(1): 12-13. 
Peterson, SC, Eberl, M, Vagnozzi, AN, Belkadi, A, Veniaminova, NA, Verhaegen, ME, Bichakjian, CK, Ward, NL, Dlugosz, AA and Wong, SY (2015). "Basal Cell Carcinoma Preferentially Arises from Stem Cells within Hair Follicle and Mechanosensory Niches." Cell Stem Cell 16(4): 400-412.

Petrova, R and Joyner, AL (2014). "Roles for Hedgehog signaling in adult organ homeostasis and repair." Development 141(18): 3445-3457.

Rahmouni, S, Cerignoli, F, Alonso, A, Tsutji, T, Henkens, R, Zhu, C, Louisdit-Sully, C, Moutschen, M, Jiang, W and Mustelin, T (2006). "Loss of the VHR dual-specific phosphatase causes cell-cycle arrest and senescence." Nat Cell Biol 8(5): 524-531.

Ramachandran, I, Ganapathy, V, Gillies, E, Fonseca, I, Sureban, SM, Houchen, CW, Reis, A and Queimado, L (2014). "Wnt inhibitory factor 1 suppresses cancer stemness and induces cellular senescence." Cell Death Dis 5: e1246.

Ramachandran, I, Thavathiru, E, Ramalingam, S, Natarajan, G, Mills, WK, Benbrook, DM, Zuna, R, Lightfoot, S, Reis, A, Anant, S and Queimado, L (2012). "Wnt inhibitory factor 1 induces apoptosis and inhibits cervical cancer growth, invasion and angiogenesis in vivo." Oncogene 31(22): 2725-2737.

Reguart, N, He, B, Xu, Z, You, L, Lee, AY, Mazieres, J, Mikami, I, Batra, S, Rosell, R, McCormick, F and Jablons, DM (2004). "Cloning and characterization of the promoter of human Wnt inhibitory factor-1." Biochem Biophys Res Commun 323(1): 229-234.

Reifenberger, J, Wolter, M, Knobbe, CB, Kohler, B, Schonicke, A, Scharwachter, C, Kumar, K, Blaschke, B, Ruzicka, T and Reifenberger, G (2005). "Somatic mutations in the PTCH, SMOH, SUFUH and TP53 genes in sporadic basal cell carcinomas." $\mathrm{Br} J$ Dermatol 152(1): 43-51.

Roberg-Larsen, H, Strand, MF, Krauss, S and Wilson, SR (2014). "Metabolites in vertebrate Hedgehog signaling." Biochem Biophys Res Commun 446(3): 669-674.

Robinson, JK and Dahiya, M (2003). "Basal cell carcinoma with pulmonary and lymph node metastasis causing death." Arch Dermatol 139(5): 643-648.

Rubin, Al, Chen, EH and Ratner, D (2005). "Basal-cell carcinoma." N Engl J Med 353(21): 2262-2269.

Ruvinsky, I and Meyuhas, O (2006). "Ribosomal protein S6 phosphorylation: from protein synthesis to cell size." Trends Biochem Sci 31(6): 342348.

Salto-Tellez, M, Peh, BK, Ito, K, Tan, SH, Chong, PY, Han, HC, Tada, K, Ong, WY, Soong, R, Voon, DC and Ito, $Y$ (2006). "RUNX3 protein is overexpressed in human basal cell carcinomas." Oncogene 25(58): 7646-7649.

Samuels, IS, Karlo, JC, Faruzzi, AN, Pickering, K, Herrup, K, Sweatt, JD, Saitta, SC and Landreth, GE (2008). "Deletion of ERK2 mitogen- 
activated protein kinase identifies its key roles in cortical neurogenesis and cognitive function." J Neurosci 28(27): 6983-6995.

Samuels, ML, Weber, MJ, Bishop, JM and McMahon, M (1993). "Conditional transformation of cells and rapid activation of the mitogen-activated protein kinase cascade by an estradiol-dependent human raf- 1 protein kinase." Mol Cell Biol 13(10): 6241-6252.

Schaniel, C, Sirabella, D, Qiu, J, Niu, X, Lemischka, IR and Moore, KA (2011). "Wnt-inhibitory factor 1 dysregulation of the bone marrow niche exhausts hematopoietic stem cells." Blood 118(9): 2420-2429.

Schindelin, J, Arganda-Carreras, I, Frise, E, Kaynig, V, Longair, M, Pietzsch, T, Preibisch, S, Rueden, C, Saalfeld, S, Schmid, B, Tinevez, JY, White, DJ, Hartenstein, V, Eliceiri, K, Tomancak, P and Cardona, A (2012). "Fiji: an open-source platform for biological-image analysis." Nat Methods 9(7): 676-682.

Schluter, H, Stark, HJ, Sinha, D, Boukamp, P and Kaur, P (2013). "WIF1 is expressed by stem cells of the human interfollicular epidermis and acts to suppress keratinocyte proliferation." J Invest Dermatol 133(6): 1669-1673.

Schnidar, H, Eberl, M, Klingler, S, Mangelberger, D, Kasper, M, HauserKronberger, C, Regl, G, Kroismayr, R, Moriggl, R, Sibilia, M and Aberger, $F$ (2009). "Epidermal growth factor receptor signaling synergizes with Hedgehog/GLI in oncogenic transformation via activation of the MEK/ERK/JUN pathway." Cancer Res 69(4): 12841292.

Sewing, A, Wiseman, B, Lloyd, AC and Land, H (1997). "High-intensity Raf signal causes cell cycle arrest mediated by p21Cip1." Mol Cell Biol 17(9): 5588-5597.

Sheldahl, LC, Park, M, Malbon, CC and Moon, RT (1999). "Protein kinase C is differentially stimulated by Wnt and Frizzled homologs in a Gprotein-dependent manner." Curr Biol 9(13): 695-698.

Shtutman, M, Zhurinsky, J, Simcha, I, Albanese, C, D'Amico, M, Pestell, R and Ben-Ze'ev, A (1999). "The cyclin D1 gene is a target of the betacatenin/LEF-1 pathway." Proc Natl Acad Sci U S A 96(10): 5522-5527.

So, PL, Langston, AW, Daniallinia, N, Hebert, JL, Fujimoto, MA, Khaimskiy, Y, Aszterbaum, $M$ and Epstein, EH, Jr. (2006). "Long-term establishment, characterization and manipulation of cell lines from mouse basal cell carcinoma tumors." Exp Dermatol 15(9): 742-750.

Soleymani, AD, Scheinfeld, N, Vasil, K and Bechtel, MA (2008). "Metastatic basal cell carcinoma presenting with unilateral upper extremity edema and lymphatic spread." J Am Acad Dermatol 59(2 Suppl 1): S1-3.

Staal, FJ, Noort Mv, M, Strous, GJ and Clevers, HC (2002). "Wnt signals are transmitted through $\mathrm{N}$-terminally dephosphorylated beta-catenin." EMBO Rep 3(1): 63-68.

Staples, MP, Elwood, M, Burton, RC, Williams, JL, Marks, R and Giles, GG (2006). "Non-melanoma skin cancer in Australia: the 2002 national survey and trends since 1985." Med J Aust 184(1): 6-10. 
Surmann-Schmitt, C, Widmann, N, Dietz, U, Saeger, B, Eitzinger, N, Nakamura, Y, Rattel, M, Latham, R, Hartmann, C, von der Mark, H, Schett, G, von der Mark, K and Stock, M (2009). "Wif-1 is expressed at cartilage-mesenchyme interfaces and impedes Wnt3a-mediated inhibition of chondrogenesis." J Cell Sci 122(Pt 20): 3627-3637.

Tang, Y, Simoneau, AR, Liao, WX, Yi, G, Hope, C, Liu, F, Li, S, Xie, J, Holcombe, RF, Jurnak, FA, Mercola, D, Hoang, BH and Zi, X (2009). "WIF1, a Wnt pathway inhibitor, regulates SKP2 and c-myc expression leading to G1 arrest and growth inhibition of human invasive urinary bladder cancer cells." Mol Cancer Ther 8(2): 458-468.

Taniguchi, H, Yamamoto, H, Hirata, T, Miyamoto, N, Oki, M, Nosho, K, Adachi, Y, Endo, T, Imai, $\mathrm{K}$ and Shinomura, $\mathrm{Y}$ (2005). "Frequent epigenetic inactivation of Wnt inhibitory factor-1 in human gastrointestinal cancers." Oncogene 24(53): 7946-7952.

Taylor, MD, Zhang, X, Liu, L, Hui, CC, Mainprize, TG, Scherer, SW, Wainwright, B, Hogg, D and Rutka, JT (2004). "Failure of a medulloblastoma-derived mutant of SUFU to suppress WNT signaling." Oncogene 23(26): 4577-4583.

Tomayko, MM and Reynolds, CP (1989). "Determination of subcutaneous tumor size in athymic (nude) mice." Cancer Chemother Pharmacol 24(3): 148-154.

Trakatelli, M, Ulrich, C, del Marmol, V, Euvrard, S, Stockfleth, E and Abeni, D (2007). "Epidemiology of nonmelanoma skin cancer (NMSC) in Europe: accurate and comparable data are needed for effective public health monitoring and interventions." Br J Dermatol 156 Suppl 3: 1-7.

Uhmann, A, Dittmann, K, Nitzki, F, Dressel, R, Koleva, M, Frommhold, A, Zibat, A, Binder, C, Adham, I, Nitsche, M, Heller, T, Armstrong, V, Schulz-Schaeffer, W, Wienands, J and Hahn, H (2007). "The Hedgehog receptor Patched controls lymphoid lineage commitment." Blood 110(6): 1814-1823.

Ulloa, F, Itasaki, N and Briscoe, J (2007). "Inhibitory Gli3 activity negatively regulates Wnt/beta-catenin signaling." Curr Biol 17(6): 545-550.

van Amerongen, $R$ and Nusse, $R$ (2009). "Towards an integrated view of Wnt signaling in development." Development 136(19): 3205-3214.

van de Wetering, M, Cavallo, R, Dooijes, D, van Beest, M, van Es, J, Loureiro, J, Ypma, A, Hursh, D, Jones, T, Bejsovec, A, Peifer, M, Mortin, M and Clevers, $\mathrm{H}$ (1997). "Armadillo coactivates transcription driven by the product of the Drosophila segment polarity gene dTCF." Cell 88(6): 789-799.

Vantaggiato, C, Formentini, I, Bondanza, A, Bonini, C, Naldini, L and Brambilla, R (2006). "ERK1 and ERK2 mitogen-activated protein kinases affect Ras-dependent cell signaling differentially." J Biol 5(5): 14.

Vassallo, I, Zinn, P, Lai, M, Rajakannu, P, Hamou, MF and Hegi, ME (2015). "WIF1 re-expression in glioblastoma inhibits migration through attenuation of non-canonical WNT signaling by downregulating the IncRNA MALAT1." Oncogene. 
Vidal, D, Matias-Guiu, X and Alomar, A (2004). "Efficacy of imiquimod for the expression of Bcl-2, Ki67, p53 and basal cell carcinoma apoptosis." $\mathrm{Br}$ J Dermatol 151(3): 656-662.

Wang, Y (2009). "Wnt/Planar cell polarity signaling: a new paradigm for cancer therapy." Mol Cancer Ther 8(8): 2103-2109.

Watanabe, $\mathrm{N}$ and Higashida, C (2004). "Formins: processive cappers of growing actin filaments." Exp Cell Res 301(1): 16-22.

Weaver, VM, Petersen, OW, Wang, F, Larabell, CA, Briand, P, Damsky, C and Bissell, MJ (1997). "Reversion of the malignant phenotype of human breast cells in three-dimensional culture and in vivo by integrin blocking antibodies." J Cell Biol 137(1): 231-245.

Wei, C, Peng, B, Han, Y, Chen, WV, Rother, J, Tomlinson, GE, Boland, CR, Chaussabel, M, Frazier, ML and Amos, Cl (2015). "Mutations of HNRNPA0 and WIF1 predispose members of a large family to multiple cancers." Fam Cancer 14(2): 297-306.

Weidinger, G and Moon, RT (2003). "When Wnts antagonize Wnts." J Cell Biol 162(5): 753-755.

Wendel, HG, De Stanchina, E, Fridman, JS, Malina, A, Ray, S, Kogan, S, Cordon-Cardo, C, Pelletier, J and Lowe, SW (2004). "Survival signalling by Akt and elF4E in oncogenesis and cancer therapy." Nature 428(6980): 332-337.

Woodson, EN and Kedes, DH (2012). "Distinct roles for extracellular signalregulated kinase 1 (ERK1) and ERK2 in the structure and production of a primate gammaherpesvirus." J Virol 86(18): 9721-9736.

Xie, J, Aszterbaum, M, Zhang, X, Bonifas, JM, Zachary, C, Epstein, E and McCormick, F (2001). "A role of PDGFRalpha in basal cell carcinoma proliferation." Proc Natl Acad Sci U S A 98(16): 9255-9259.

Xie, J, Murone, M, Luoh, SM, Ryan, A, Gu, Q, Zhang, C, Bonifas, JM, Lam, CW, Hynes, M, Goddard, A, Rosenthal, A, Epstein, EH, Jr. and de Sauvage, FJ (1998). "Activating Smoothened mutations in sporadic basal-cell carcinoma." Nature 391(6662): 90-92.

Yamamoto, T, Ebisuya, M, Ashida, F, Okamoto, K, Yonehara, S and Nishida, E (2006). "Continuous ERK activation downregulates antiproliferative genes throughout G1 phase to allow cell-cycle progression." Curr Biol 16(12): 1171-1182.

Yang, SH, Andl, T, Grachtchouk, V, Wang, A, Liu, J, Syu, LJ, Ferris, J, Wang, TS, Glick, AB, Millar, SE and Dlugosz, AA (2008). "Pathological responses to oncogenic Hedgehog signaling in skin are dependent on canonical Wnt/beta3-catenin signaling." Nat Genet 40(9): 1130-1135.

Yao, S, Lum, L and Beachy, P (2006). "The ihog cell-surface proteins bind Hedgehog and mediate pathway activation." Cell 125(2): 343-357.

Yarden, $Y$ and Pines, G (2012). "The ERBB network: at last, cancer therapy meets systems biology." Nat Rev Cancer 12(8): 553-563.

Yarden, $Y$ and Sliwkowski, MX (2001). "Untangling the ErbB signalling network." Nat Rev Mol Cell Biol 2(2): 127-137.

Yee, DS, Tang, Y, Li, X, Liu, Z, Guo, Y, Ghaffar, S, McQueen, P, Atreya, D, Xie, J, Simoneau, AR, Hoang, BH and Zi, X (2010). "The Wnt 
inhibitory factor 1 restoration in prostate cancer cells was associated with reduced tumor growth, decreased capacity of cell migration and invasion and a reversal of epithelial to mesenchymal transition." Mol Cancer 9: 162.

Yoshikawa, S, McKinnon, RD, Kokel, M and Thomas, JB (2003). "Wntmediated axon guidance via the Drosophila Derailed receptor." Nature 422(6932): 583-588.

Youssef, KK, Lapouge, G, Bouvree, K, Rorive, S, Brohee, S, Appelstein, O, Larsimont, JC, Sukumaran, V, Van de Sande, B, Pucci, D, Dekoninck, S, Berthe, JV, Aerts, S, Salmon, I, del Marmol, V and Blanpain, C (2012). "Adult interfollicular tumour-initiating cells are reprogrammed into an embryonic hair follicle progenitor-like fate during basal cell carcinoma initiation." Nat Cell Biol 14(12): 1282-1294.

Youssef, KK, Van Keymeulen, A, Lapouge, G, Beck, B, Michaux, C, Achouri, Y, Sotiropoulou, PA and Blanpain, C (2010). "Identification of the cell lineage at the origin of basal cell carcinoma." Nat Cell Biol 12(3): 299305.

$\mathrm{Yu}, \mathrm{H}$, Mouw, JK and Weaver, VM (2011). "Forcing form and function: biomechanical regulation of tumor evolution." Trends Cell Biol 21(1): 47-56.

Zhang, J, Gill, AJ, Issacs, JD, Atmore, B, Johns, A, Delbridge, LW, Lai, R and McMullen, TP (2012). "The Wnt/beta-catenin pathway drives increased cyclin D1 levels in lymph node metastasis in papillary thyroid cancer." Hum Pathol 43(7): 1044-1050.

Zibat, A, Uhmann, A, Nitzki, F, Wijgerde, M, Frommhold, A, Heller, T, Armstrong, V, Wojnowski, L, Quintanilla-Martinez, L, Reifenberger, J, Schulz-Schaeffer, W and Hahn, H (2009). "Time-point and dosage of gene inactivation determine the tumor spectrum in conditional Ptch knockouts." Carcinogenesis 30(6): 918-926. 


\section{VII}

Abbreviations

\#

$\%$

\&

‘

"

o

$\mu$

$\infty$

$+$

$+$

$-$

A

$A b$

ad libitum

AEC

$A G$

Akt

Ankrd

Apc

a.u.

$A_{x x x}$

b

BAD

BCC

$\mathrm{Bcl} 2$

Bcl9

BCNS

bFGF number

percent

and

minute

second

degree

micro

unlimited

positive

wild-type

minus

knockout

Ampere

antibody

lat. at liberty

3-amino-9 ethylcarbazole

stock company (german: Aktiengesellschaft)

$\mathrm{v}$-akt murine thymoma viral oncogene homolog

ankyrin repeat domain

adenomatosis polyposis coli

arbitrary unit

absorbance at $x x x \mathrm{~nm}$

y-intercept

BCL2 antagonist of cell death

basal cell carcinoma

B cell leukemia/lymphoma 2

B cell CLL/lymphoma 9

basal cell nevus syndrome

basic fibroblastic growth factor 
Boc

biregional cell adhesion molecule-related/down-

Boi regulated by oncogenes (Cdon) binding protein

bp brother of Ihog

BrdU

BSA

base pair

5-bromo-2'-deoxyuridine

C

bovine serum albumin

C

C

$\mathrm{Ca}^{2+}$

$\mathrm{CaCl}_{2}$

CAFs

CaMKII

Celsius

centi

concentration

calcium

calcium chloride

cancer-associated fibroblasts

calcium/calmodulin-dependent protein kinase II

Cbp

CREB-binding protein

CD

cluster of differentiation

Cdh

cadherin

CDK

cyclin-dependent kinase

cDNA

complementary DNA

Cdo1

$\mathrm{Ci}$

cysteine dioxygenase 1 , cytosolic

cubitus interruptus

CM

conditioned medium

CMV

cytomegalovirus

Cn

calcinurin

Co.

$\mathrm{CO}_{2}$

company

carbon dioxyde

Cre

Creb

causes recombination

Csnk1a1

cAMP responsive element binding protein

Ct

casein kinase 1 , alpha 1

threshold cycle

C-terminal

carboxyterminal

Daam1

Dvl associated activator of morphogenesis 1

dATP

2'-deoxyadenosine 5'-triphosphate 
DAG

DB

dCTP

$\mathrm{ddH} 2 \mathrm{O}$

del

dGTP

Dhh

Disp

Dkk

DMEM

DMSO

DNA

DNase

dNTP

dpc

Drl

DTT

dTTP

Dvl

E. coli

ECM

e.g.

EDTA

EGF

EGFR

EGTA

eGFP

EMT

ER

ErbB

Erk
1,2 diacylglycerol batabase

2'-deoxycytidine 5'-triphosphate

double-distilled water

deleted, deletion

2'-deoxyguanosine 5'-triphosphate

Dessert hedgehog

Dispatched

dickkopf

Dulbecco's modified eagle medium

dimethyl sulfoxide

deoxyribonucleic acid

deoxyribonuclease

deoxynucleotide

days post coitum

derailed

dithiothreitol

2'-deoxythymidine 5'-triphosphate

dishevelled

Escherichia coli

extracellular matrix

exempli gratia (lat. for example)

ethylenediaminetetraacetic acid

epidermal growth factor

epidermal growth factor receptor

ethylene glycol tetraacetic acid

enhanced GFP

epithelial-to-mesenchymal transition

endoplasmic reticulum

v-erb-b2 erythroblastic leukemia viral oncogene

homolog

extracellular-signal regulated kinase

134 


\begin{tabular}{|c|c|}
\hline ERT & tamoxifen-inducible estrogen receptor \\
\hline et al. & et alii (lat. and others) \\
\hline $\mathrm{EtOH}$ & ethanol \\
\hline FCS & fetal calf serum \\
\hline FDA & Federal Drug Agency \\
\hline Fig. & figure \\
\hline FITC & fluorescin isothiocyanate \\
\hline flox & floxed \\
\hline Fn1 & fibronectin 1 \\
\hline FOP & SuperFOPFlash expression plasmid \\
\hline FOXO & Forkhead (FKHR) family of transcription factors \\
\hline $\mathrm{Fu}$ & Fused \\
\hline Fzd & frizzled \\
\hline$g$ & gramm \\
\hline$g$ & g-force \\
\hline GAPDH & glyceraldehyde 3-phosphate dehydrogenase \\
\hline gDNA & genomic DNA \\
\hline GFP & green fluorescent protein \\
\hline Gli & Glioma-associated oncogene family member \\
\hline GliR & Gli repressor form \\
\hline GmbH \& Co KG & $\begin{array}{l}\text { limited partnership with a limited liability company as } \\
\text { general partner (german: Gesellschaft mit } \\
\text { beschränkter Haftung Compagnie und } \\
\text { Kommanditgesellschaft) }\end{array}$ \\
\hline $\mathrm{GmbH}$ & $\begin{array}{l}\text { limited liability company (german: Gesellschaft mit } \\
\text { beschränkter Haftung) }\end{array}$ \\
\hline GPCR & G-protein coupled receptor \\
\hline G-Phase & gap phase \\
\hline Gsk3 & glycogen synthase kinase 3 \\
\hline $\mathrm{h}$ & hour \\
\hline $\mathrm{H}_{2} \mathrm{O}_{2}$ & hydrogen peroxyde \\
\hline $\mathrm{HCl}$ & hydrochloric acid \\
\hline
\end{tabular}


H\&E

HEPES

HEK-293

HGF

$\mathrm{Hh}$

HNRNPAO

Hprt

HRP

HRPO

$\mathrm{Hsc70}$

HSPGs

i.e.

$\lg$

Igf2bp1

IHC

Ihh

Ihog

i.m.

Inc.

i.p.

$\mathrm{IP}_{3}$

IVI

JNK

$\mathrm{k}$

K1

K10

$\mathrm{kb}$

$\mathrm{KCl}$

$\mathrm{KH}_{2} \mathrm{PO}_{4}$

KG haematoxylen and eosin

4-(2-hydroxyethyl)-1-piperazineethanesulfonic acid human embryonic kidney cells 293

hepatocyte growth factor

Hedgehog

heterogeneous nuclear ribonucleoprotein $\mathrm{A} 0$

hypoxanthine phosphoribosyltransferase 1

horse-raddish peroxidase

horse-raddish peroxidase

heat-shock protein 70

heparan sulfate proteoglycans

id est (lat. that is)

immunoglobulin

insulin-like growth factor 2 mRNA binding protein 1

immunohistochemistry

Indian hedgehog

interference hedgehog

intramuscular

incorporated

intraperitoneal

inositol 1,4,5-triphosphate

involucrin

c-Jun $\mathrm{N}$-terminal kinase

kilo

keratin 1

keratin 10

kilobase

potassium chloride

monopotassium phosphate

limited Partnership (german:

Kommanditgesellschaft) 
I

LacZ

liter

LAR II

$\beta$-Galactosidase

LB

Luciferase Assay Reagent II

Lef

lysogeny broth

$\mathrm{LiCl}$

lymphoid enhancer factor

$\log$

$\mathrm{LOH}$

Lor

Lrp

Ltd.

m

m

$\mathrm{m}$

m

M

Map

Mapk

Map3k

MB

MEF

Mek

$\mathrm{MgCl}_{2}$

$\mathrm{MetOH}$

$\min$

mRNA

MSCV

mTOR

Myc

$\mathrm{n}$

$\mathrm{Na}$

$\mathrm{Na}_{2} \mathrm{HPO}_{4}$

lithium chloride

logarithm

loss-of -heterozygosity

loricrin

LDL receptor-related protein

limited

meter

milli

murine

slope

molar

Mitogen-activated protein

Mitogen-activated protein kinase

Mitogen-activated protein kinase kinase kinase

medulloblastoma

mouse embryonic fibroblast

MAPK/ERK kinase

magnesium dichloride

methanol

minute

messenger ribonucleic acid

murine stem cell virus

mammalian target of rapamycin

myelocytomatosis oncogene

nano

sodium

disodium hydrogen phosphate

137 
$\mathrm{NaCl}$

Nfat

$\mathrm{Nf}-\mathrm{kB}$

$\mathrm{NIH}$

NMSC

n.s.

$\mathrm{N}$-terminal

OD

$\mathrm{O} / \mathrm{N}$

$\mathrm{p}$

$p$

$p$

PBS

PBST

PCR

PCP

PDGF

PDGFR

$\mathrm{pH}$

PI

PI3K

$\mathrm{PIP}_{3}$

$\mathrm{Pk}$

PKC

PLB

PLC

POD

Porcn

PS

PSMF

Ptch sodium chloride

nuclear factor associated with $T$ cells

kappa light polypeptide gene enhancer in B cells

National Institutes of Health

non-melanoma skin cancer

not significant

aminoterminal

optical density

overnight

pico

plasmid

phospho

Phosphate-buffered sodium chloride-solution

Phosphate-buffered sodium chloride-solution with

Tween-20

polymerase chain reaction

planar cell polarity

platelet derived growth factor

platelet derived growth factor receptor

lat. potentia hydrogenii

propidium iodide

phosphatidylinositol-4,5-bisphosphate 3-kinase

phosphatidylinositol-3,4,5-triphosphate

prickle

protein kinase $\mathrm{C}$

passive lysis buffer

protein lipase $\mathrm{C}$

peroxidase

palmitoyltransferase porcupine

penicillin/streptomycin

phenylmethanesulfonylfluoride

patched1 
Ptch2

Ptk7

Puro

Pygo

qRT-PCR

Raf

Ras

RIPA buffer

Rlu

RMS

RNA

RNase

Rock

Ror

rRNA

RT

RYK

S6

SAPK

SCC

SDS

Sdc-2

$\mathrm{sec}$

SEM

Sfrp

Shh

Sma

Smo

SmoM2

SN

SOC patched2

protein tyrosine kinase 7

puromycin

pygopus

quantitative real-time PCR

rat sarcoma virus oncogene homolog

radioimmunoprecipitation assay buffer

relative light units

rhabdomyosarcoma

ribonucleic acid

ribonuclease

Rho-associated coiled-coil containing protein kinase receptor tyrosine kinase-like orphan receptor

ribosomal RNA

room temperature

receptor tyrosine kinase

ribosomal protein S6

stress-activated phospho-kinases

squamous cell carcinoma

sodium dodecyl sulfate

syndecan-2

second

standard error of the mean

secreted frizzled-related protein

sonic hedgehog

smooth muscle actin

smoothened

constitutively active W539L point mutation of the mouse smoothened homolog gene supernatant

super optimal broth with catabolite repression 139 
SOS

SPF

S-Phase

St.

STAT

stauro

Sufu

SYBR

TAMs

Taq

TBE

Tbp

TBS

TBST

Tcf

TE

TF

TGF- $\beta$

Tgm1

TK

Tle

TOP

TP53

$\beta$-TrCP

Tris

TUNEL

U

USA

UV

V

v son of sevenless

specified pathogen-free

synthesis phase

saint (german: Sankt)

signal transducer and activator of transcription

staurosporine

suppressor of fused homolog

Synergy Brands Inc.

tumor-associated macrophages

thermos aquaticus

Tris-boric acid-EDTA-solution

TATA box binding protein

Tris-buffered sodium chloride-solution

Tris-buffered sodium chloride-solution containing

Tween-20

T-cell factor

Tris/EDTA buffer

transcription factor

Transforming growth factor beta

transglutaminase 1

thymidine kinase

transducin-like enhancer of split

SuperTOPFlash expression plasmid

tumor protein p53

beta-transducin repeat containing protein

tris(hydroxymethyl)aminomethane

TdT-mediated dUTP-biotin nick end labeling

unit

United States of America

ultraviolet

Volt

volume 


$\begin{array}{ll}\text { Vangl } & \text { vang-like } \\ \text { VEGF } & \text { vascular endothelial growth factor } \\ \text { vs } & \text { versus } \\ \text { W } & \text { Watt } \\ \text { w } & \text { weight } \\ \text { WD } & \text { WIF domain } \\ \text { WHO } & \text { World Health Organization } \\ \text { Wif1 } & \text { Wnt inhibitory factor 1 } \\ \text { wk } & \text { week } \\ \text { Wls } & \text { wntless } \\ \text { Wnt } & \text { wingless-type MMTV integration site family } \\ \text { WST-1 } & \text { water soluble tetrazolium salt } 1 \\ \text { wt } & \text { wild-type } \\ \text { x } & \text { fold }\end{array}$




\section{Acknowledgements}

This thesis would not have been possible without the support and aid of all the people which accompanied me on my way to complete this study. First of all I would like express my endless gratitude to my supervisor Prof. Dr. Heidi Hahn. Thank you for your support, continuous mentoring, numerous discussions and proofreading of this thesis. Thank you for giving me the opportunity to work on this exciting project.

Many thanks also to the members of my thesis committee Prof. Dr. Matthias Dobbelstein and Prof. Dr. Tobias Pukrop for your helpful input and numerous ideas.

Special thanks go to Dr. Kai Dittmann for all your support and advice in all the experiment requiring flow cytometry and for the joyful small talks that made my visits in the department of immunology a pleasant stay.

A big thank to Prof Dr. Walter Schulz-Schaeffer for the interpretation of all my antibody stainings.

I also would like to thank Prof. Dr. Michael Schön for his support and for providing human tissue samples.

Special thanks also go to the animal care takers, especially Susann Peter and Jennifer Flemming for the excellent animal care and being a memory aid when my animals desperately needed to "retire".

Countless thanks go to the people of the lab I loved to work with, many of them became more than colleagues. In particular, I would like to thank Dr. Frauke Nitzki for your patients and guidance in the very beginning of this project and Benedikt Linder for always having an open ear, for all the discussions, BBQs and hours in the gym to forget the lab for a while. Moreover, I would like to thank Anke Frommhold, Ina Heß and Eva Stetter for your great support and backup during all the time. You rock! Many Thanks also to all the other current and former lab members, Dr. Anja Uhmann, Dr.Simone König, Dr. Diana Rettberg, Dr. Penelope Pelczar, Rosalie Ridzewski, Julia Dräger, Joana Pyczek, Nicole Cuvelier and Tobias Goldak 
for your support and making this a wonderful experience. It was great to work with all of you.

I would also like to thank Prof. Peter Burfeindt and Dr. Silke Kaulfuß for adopting me for lunch and all the funny moments and discussion during all the years.

Especially, I would like to thank my friends and my parents Ingrid and Herbert, to whom I would like to dedicate this thesis, for their endless support and encouragement during my studies and my thesis. I would not have made it without you. Thank you with all my heart!

Finally, I would like to express special thanks to Pia who supported me throughout the critical phase. Thank you for your patience and your warmness. 


\title{
IX Curriculum vitae
}

\author{
Personal information \\ Name: Marco Becker \\ Date of Birth: 23.07.1985 \\ Place of Birth: Gelsenkirchen, Germany \\ Citizenship: German
}

01/2012-09/2015 Research assistant (Ph. D. candidate), Georg-August-University Göttingen

University Medical Center Göttingen; Institute of Human Genetics, Tumor Genetics group

Within the Göttingen Graduate School for Neurosciences, Biophysics and Molecular Biosciences (GGNB) and the doctoral program Molecular biology of cells that is part of GAUSS

(Georg-August University School of Science)

Thesis title: "Wif1 Inhibits the Growth of Basal Cell Carcinoma"

10/2009-01/2012 M.Sc., Georg-August-University Göttingen

Within the master program "Developmental, Neural and Behavioral Biology" with focus on "Cell and Developmental Biology".

Thesis title: "The Role of Wif1 in Basal Cell Carcinoma"

$10 / 2006-07 / 2009$

\section{B.Sc., Georg-August-University Göttingen}

Within the bachelor program "Biology" with focus on "Molecular Biosciences".

Thesis title: "Aspects of the Putative Pluripotency Gene Stra8"

$07 / 2005$

Abitur, Gymnasium-Petrinum Dorsten.

\section{Publications}

Frauke Nitzki, Marco Becker, Anke Frommhold, Walter Schulz-Schaeffer and Heidi Hahn (2012) "Patched Knockout Mouse Models of Basal Cell Carcinoma”; Journal of Skin Cancer, Volume 2012 (2012), Article ID 907543 